\title{
2nd International Congress on Neurology and Epidemiology
}

Nice, France, November 8-10, 2012

\section{Abstracts}

\section{Editors}

V.L. Feigin, Auckland, New Zealand

M. Giroud, Dijon, France 


\section{Contents}

\section{Plenary Sessions}

K1 - Keynote Lecture 1

K2 - Keynote Lecture 2

PS1 - New Frontiers in Neuroepidemiology

PS2 - Stroke Care and Prevention

PS3 - Treatment and Neurorehabilitation

PS4 - Dementia and Aging

PS5 - Translational Neuroscience: A Practical Approach

PS6 - Updates on Parkinson's Disease

PS7 - Epilepsy \& Neurodegenerative Disorders

PS8 - ICD-11: Implications for Neurology

PS9 - Rare Neurogenetic Disease: Practical Approach

PS10 - Methodological Issues in Neuroepidemiology

PS11 - Updates on Multiple Sclerosis

PS12 - Controversies in the Management of Neurological Disorders

PS13 - Alternative Medicine and Rehabilitation In Neurological Disorders

SS1 - Satellite Symposium of the WFN "What Is an Environmental Factor?"

\section{Poster Presentations}

179 P1 - Prevention 228

179 P2 - Diagnosis 229

179 P3 - Epidemiology 235

180 P4 - Treatment/Management 258

181 P5 - Neurosurgery 262

182 P6 - Neurorehabilitation 263

P7 - Neuroimaging Studies 266

183 P8 - Neurogenetics 267

184 P9 - Neuropharmacology 269

185 P10 - Neuropsychology 272

186 P11 - Neuropsychiatry 273

P12 - Basic and Translational Neuroscience 274

187 P13 - Nursing Care 275

188 P16 - Healthcare 276

189 P17 - Ethnicity Issues 277

P18 - Public Health 278

190

Author Index

279

\section{Oral Presentations}

O1 - Prevention 192

O2 - Diagnosis 195

O3 - Epidemiology 195

O4 - Treatment/Management 217

O5 - Neurosurgery 220

O7 - Neuroimaging Studies 221

O8 - Neurogenetics 222

O9 - Neuropharmacology 224

O10 - Neuropsychology 224

011 - Neuropsychiatry 225

012 - Basic and Translational Neuroscience 226

O15 - Health Economics 226

O18 - Public Health 227 


\section{Keynote Lecture 1}

\section{Theory of Reality}

Wiebers D.O.

Mayo Clinic, USA

This presentation combines key elements of neuroscience, physics and metaphysical science to provide the foundation for a new knowledge base which allows a deeper understanding of the nature of consciousness and the nature of reality as we experience it. The core identity of each individual relative to the body and brain is key since the fundamental nature of individual consciousness is the same as the fundamental nature of the universe. In 2005, the journal Science published a special anniversary issue delineating 125 questions that scientists have so far failed to answer. The most important unanswered question was: "What is the universe made of?" and the second most important was: "What is the biological basis of consciousness?" The Theory of Reality provides a basis for answering these questions.

\section{Keynote Lecture 2}

\section{Western Pacific ALS-PDC: A Prototypical Neurodegenerative Disease with a Dominant Environmental Etiology}

Spencer $P$.

Oregon Health \& Science University, USA

Amyotrophic lateral sclerosis (ALS) and parkinsonismdementia complex (PDC), a single nosologic entity (polyproteinopathy dominated by tau) with a variety of clinical forms, has been present in high incidence in three genetically distinct island populations in the Marianas (Chamorros of Guam and Rota), Honshu (Japanese of Kii Peninsula) and New Guinea (Auyu and Jaqai linguistic groups of West Papua). Studies of ALS-PDC have shown an increasing age of disease appearance, changing clinical patterns (from ALS to parkinsonism-dementia), an absence of genetic mutations causal of well known neurodegenerative disorders, and a declining disease prevalence that is consistent with a disappearing environmental factor common to the three affected populations. Epidemiological studies on Guam have repeatedly implicated food use of the seed of the cycad plant (Cycas micronesica), while medicinal use of its neurotoxic seed by individuals who later developed ALS has been demonstrated in Guam, Kii, and West Papua. Cycads contain cycasin, the aglycone (methyl- azoxymethanol, MAM) of which is a potent genotoxin, carcinogen, and neurotoxin, with confirmed epidemiological links to both ALS and parkinsonism-dementia on Guam. The neurotoxic property of MAM arises from unrepaired DNA damage (O6-methylguanine), which activates intracellular pathways leading to cancer in cycling cells and to degenerative changes in postmitotic neurons. Cycads also contain the neuroexcitant beta-N-methylamino-L-alanine (L-BMAA), an amino acid found as a ubiquitous product of cyanobacteria in soil, freshwater and marine environments and, consequently, present in filter feeders used as seafood. L-BMAA is taken up by brain tissue as a proteinassociated foreign amino acid, which raises the possibility of neuroprotein conformational changes that promote neuropathology or even trigger prion-like neurodegeneration. Taken together, continued study of western Pacific ALS-PDC is providing new insights into the environmental origins, nosologic associations, and molecular mechanisms of neurodegenerative disease.

\section{PS1: New Frontiers in Neuroepidemiology}

\section{PS1-1 \\ Imaging Genetics: The New Frontier in Research on Dementia}

Ikram A.

Erasmus MC, The Netherlands

Alzheimer's disease is a multi-factorial disease involving different pathologies that steadily accumulate in the brain during life. Initially, the presence of such pathologies remains subclinical and can only be detected using various imaging modalities and by sampling biospecimens, such as cerebrospinal fluid and serum. Only after a certain threshold has been passed do clinical signs and symptoms develop, ultimately leading to the clinical diagnosis of dementia due to Alzheimer's disease. Genetic research has been an important cornerstone in etiologic research on Alzheimer's disease. Family studies have repeatedly shown strong heritability of Alzheimer's disease, with heritability estimated to be between 60 and $80 \%$. Against this background, linkage studies have long established APP, PSEN1, PSEN2 as mendelian genes leading to familial Alzheimer's disease, which accounts for less than $5 \%$ of all cases. The discovery of APOE denoted the first gene to be involved in the more common non-mendelian form of Alzheimer's disease. For almost two decades, APOE remained the only robustly replicated gene for sporadic Alzheimer's disease. Recently, the advent

\begin{tabular}{ll}
\hline KARGER & ( ) 2012 S. Karger AG, Basel \\
Fax +41613061234 & 0251-5350/12/0394-0179\$38.00/0 \\
$\begin{array}{l}\text { E-Mail karger@karger.ch } \\
\text { www.karger.com }\end{array}$ & $\begin{array}{l}\text { Accessible online at: } \\
\text { www.karger.com/ned }\end{array}$
\end{tabular}


of genome-wide association studies (GWAS) has revolutionized the genetics of various complex diseases, including Alzheimer's disease. This talk aims to provide an overview of the developments in genetic epidemiology of sporadic Alzheimer's disease over the last 5 years. A specific focus will be on a novel field of research that aims to combine the hypothesis-free genome-wide approach with state-of-the-art MR-imaging techniques, including voxel-based analysis. Funding was obtained through the Netherlands Organization for Health Research and Development (ZonMW Veni no: 016.136.054 to dr. Ikram).

\section{PS1-2}

\section{Contributions of the Rochester Epidemiology Project to Neurology}

Rocca W.

Mayo Clinic, USA

Background: Comprehensive and long-term health care information systems are rare in the United States and worldwide.

Methods: The Rochester Epidemiology Project (REP) recordslinkage system was established in 1966 to capture health care information for the entire population of Olmsted County, Minnesota, USA (www.rochesterproject.org). The REP includes a dynamic cohort of 502, 820 unique individuals who resided in Olmsted County at some point between 1966 and 2010 and received health care for any reason at a healthcare provider in the county. The system covers residents of all ages, of both sexes, and regardless of socioeconomic status, ethnicity, or insurance status.

Results: The data available electronically include demographic characteristics, medical diagnostic codes, surgical procedure codes, and death data (including causes of death). In addition, the system keeps a complete list of all paper records, electronic records, and scanned documents available for more in-depth review and abstraction. The REP serves as the research infrastructure for studies of virtually all diseases that come to medical attention, and has supported over 2000 peer-reviewed publications since 1966.

Conclusions: Common uses of the REP are incidence or prevalence studies, case-control studies, cohort studies, cost or costeffectiveness studies, and natural history or outcomes studies. The REP has supported many studies of neurological diseases, particularly of common diseases such as dementia, Parkinson's disease, stroke, and epilepsy.

\section{PS2: Stroke Care and Prevention}

\author{
PS2-1 \\ Obesity and Stroke: Does the Size Matter? \\ INTERSTROKE Study and Literature Overview
}

O'Donnell

Ireland

Numerous studies have documented a strong association between measures of obesity and risk of stroke. In addition, obesity is an important risk factor for diabetes mellitus, dyslipidemia and hypertension, and determinants of obesity (e.g. diet and exercise) are also independent risk factors for stroke. In the INTERSTROKE Phase 1 case-control study, which included 6, 000 participants from 22 countries, we found that increasing waist-to-hip was a risk factor for both ischemic stroke and intracerebral hemorrhage. Compared to the lowest tertile of waist-to-hip ratio, there was a graded increase in the risk of for all stroke (OR 1.42 ; 99\%CI 1.18-1.71 for second tertile, and OR 1.65; 99\%CI $1.36-1.99)$ on multivariable analyses. The estimate populationattributable risk was $26.5 \%$ (99\%CI 18.8-36.0) for all-stroke in the entire study. The INTERSTROKE study also observed that waistto-hip ratio had a stronger association with stroke risk, compared to body mass index (BMI). Accordingly, prevention and management of obesity represents a key target for stroke prevention, at an individual and population-level. Phase 2 of INTERSTROKE ( $\mathrm{n}=$ $25,000)$ will determine whether there are regional variations in the magnitude of importance of obesity, and genetic determinants that may modify the association between obesity and stroke.

\section{PS2-2}

\section{Stroke Issues in a Developing Country}

Banerjee T., Das S.

National Neurosciences Centre Calcutta, India

Globally, stroke is the 2nd commonest cause of mortality after coronary artery disease and a leading cause of adult disability. More than 4/5th of all stroke occur in developing countries. In many Western industrialized countries, the stroke incidence has fallen over the past few decades with the present annual incidence rates (AIR) between 58 and 88 per 100, 000 persons and 1-month case fatality rates (CFR) $8-22 \%$. In India, on the contrary, incidence rate of stroke has risen exponentially during this period. The current AIR of stroke is $135-152$ per 100,000 persons and CFR $27-41 \%$, in India. The stroke prevalence is about 550 per 100 , 000. Regarding stroke subtypes, the ischemic: hemorrhagic stroke ratio is 2 to $3.5: 1$, indicating proportionately higher prevalence of cerebral hemorrhage in India than in the Western nations. Intracranial large artery atherosclerosis is the commonest underlying factor for ischemic stroke in India. Among young adults rheu- 
matic heart disease, tuberculous endarteritis, Moya-Moya disease and among young women cerebral venous thrombosis are important causes for ischemic stroke. The common risk factors among Indians include hypertension, diabetes mellitus, smoking and central obesity. Elevated lipoprotein (a) and homocystein as risk factors also exist in many. Modern management of acute stroke including thrombolysis is expensive and not affordable to many. Hence attention should primarily be paid on the preventive measure, namely, monitoring of blood pressure, control of hypertension/ diabetes mellitus, abstinence from smoking and restriction of dietary salt.

\section{PS2-3}

\section{Usefulness of National Stroke Surveys}

Bornstein N.

Tel-Aviv Sourasky Medical Center, Israel

Globally, stroke is the 2nd commonest cause of mortality after coronary artery disease and a leading cause of adult disability. More than 4/5th of all stroke occur in developing countries. In many Western industrialized countries, the stroke incidence has fallen over the past few decades with the present annual incidence rates (AIR) between 58 and 88 per 100, 000 persons and 1-month case fatality rates (CFR) $8-22 \%$. In India, on the contrary, incidence rate of stroke has risen exponentially during this period. The current AIR of stroke is $135-152$ per 100,000 persons and CFR $27-41 \%$, in India. The stroke prevalence is about 550 per 100 , 000. Regarding stroke subtypes, the ischemic: hemorrhagic stroke ratio is 2 to 3.5:1, indicating proportionately higher prevalence of cerebral hemorrhage in India than in the Western nations. Intracranial large artery atherosclerosis is the commonest underlying factor for ischemic stroke in India. Among young adults rheumatic heart disease, tuberculous endarteritis, Moya-Moya disease and among young women cerebral venous thrombosis are important causes for ischemic stroke. The common risk factors among Indians include hypertension, diabetes mellitus, smoking and central obesity. Elevated lipoprotein (a) and homocystein as risk factors also exist in many. Modern management of acute stroke including thrombolysis is expensive and not affordable to many. Hence attention should primarily be paid on the preventive measure, namely, monitoring of blood pressure, control of hypertension/ diabetes mellitus, abstinence from smoking and restriction of dietary salt.

\section{PS3: Treatment and Neurorehabilitation}

\section{PS3-1 \\ Updates on Essential Tremor: Emerging Clinical, Epidemiological and Biological Insights into One of the Most Common Neurological Diseases}

Louis E.

Columbia University, USA

Background: Essential tremor (ET) is one of the most prevalent neurological diseases, with a prevalence (all ages) $=0.9 \%$. Prevalence increases markedly with age, and especially with advanced age (e.g., 6.3\% among those age 65 and older and as high as $21 \%$ among those age 95 and older). Despite this, ET is among the most poorly understood and most commonly misunderstood neurological disorders.

Methods: The author reviews the results of recent clinical, epidemiological and biological studies that are transforming our understanding of this enigmatic neurological disease.

Results: Although viewed in the past as a clinically-bland, monosymptomatic entity, more rigorous clinical research over the past decade indicates that the disease is characterized by a variety of motor as well as non-motor features. These motor findings include not only a range of tremors but also gait abnormalities. The non-motor features include cognitive changes (ranging from mild to severe), apathy, depression, and changes in sleep. The mood disturbance may be primary, preceding the motor manifestations. Several studies have also observed a distinctive personality profile; this could have implications for treatment. Although known to be progressive, there is a dearth of longitudinal data. Recent data are beginning to quantify for the first time the rate of progression of tremor over time. Etiological studies have yet to identify an ET gene despite the clear presence of families with multiple affected individuals over several generations. Several toxic/environmental etiologies have been studied, including harmane (1-methyl-9H-pyrido[3, 4-b]indole), a beta-carboline alkaloid with tremor-producing properties. Two studies in New York have indicated that blood harmane levels are elevated in ET cases compared to controls, but these observations were cross-sectional, assessing each subject at only one time point. In a recent study in New York, blood harmane concentration was elevated in ET cases compared to controls when reassessed at a second time point several years later, indicating what seems to be a stable association between this environmental toxin and ET. Concentrations were highest in ET cases with family history of ET. Similarly, a recent cross-sectional study in Spain indicated that blood harmane levels were slightly elevated in a group of familial ET cases compared to a group of controls in Spain, extending observations from New York to a second cohort of ET cases in Spain. The underlying biology of ET is not well understood. In the past, the disorder was viewed as a physiological disarrangement due to proposed abnormalities in olivary-cerebellar circuits. However, there are few if any empirical data to support this somewhat outdated disease model. More recently, and based 
on controlled postmortem studies, the disease is being regarded as perhaps one of cerebellar degeneration.

Conclusion(s): Emerging data from clinical, epidemiological and biological studies are now beginning to elucidate some of the risk factors for, clinical features of, and underlying mechanisms of ET. These studies answer some questions; more importantly, they serve to open many additional avenues for research.

\section{PS3-2}

Stroke Management and Clinical Researches in China

Ming L.

Stroke Clinical Research Unit, West China Hospital, Sichuan

University, Chengdu, China

Most stroke therapies recommended by guidelines in China are similar with that in western countries. However, there are some differences. Urokinase and defibrase are recommended in Chinese guideline. Difference in culture and health system might be some explanations. Thrombolysis is underused and the lower dose of rTPA is in testing. Stenting is more commonly used than CEA and standard rehabilitation is not widely available in China. Traditional local therapies such as Herbal medicine and acupuncture have long been widely practised in China. Traditional Chinese Medicine (TCM) has played and might still play an important part in treating acute stroke patients in China unless there is reliable evidence to refute its effect. More and more good clinical studies are published recently. Future strategies for improvement of acute stroke care in China include to promote evidence based stroke guidelines development and widely implementation of these guidelines, and to conduct more high quality clinical trials for testing stroke therapies.

\section{PS4: Dementia and Aging}

\section{PS4-1}

\section{Oophorectomy, Menopause, and Estrogen Treatment} in Brain Aging

Rocca W.

Mayo Clinic, USA

Background: The role of menopause and estrogen on brain aging remains unclear.

Methods: We conducted an historical cohort study in Olmsted County, MN using the records-linkage system of the Rochester Epidemiology Project (the Mayo Clinic Cohort Study of Oophorectomy and Aging).
Results: Women who had both ovaries removed prior to reaching natural menopause experienced a long-term increased risk of cognitive impairment or dementia, parkinsonism, depressive symptoms, and anxiety symptoms. The risk was higher with younger age at oophorectomy for all outcome diseases, and was offset by estrogen therapy following the surgery for cognitive impairment or dementia but not for the other outcomes.

Conclusions: Because these associations were observed in a cohort study, we cannot exclude that they may have resulted from the confounding effect of genetic variants or of other risk factors. However, the evidence for a confounding effect is limited. Therefore, we also suggest three possible causal mechanisms: 1) the associations may be mediated by an abrupt reduction in levels of circulating estrogen; 2) the associations may be mediated by an abrupt reduction in levels of circulating progesterone or testosterone; and 3) the associations may be mediated by the increased release of gonadotropins by the pituitary gland in response to the loss of circulating ovarian hormones. These three proposed causal mechanisms are probably not mutually exclusive, and may play different roles in different women (heterogeneity of effects at the population level).

\section{PS4-2 \\ White Matter Lesions/Silent Infarcts and the Risk of Dementia}

Tzourio C.

Inserm U708, France

Over the past 20 years there has been numerous studies showing the importance of ischemic cerebral lesions, mainly white matter lesions and silent infarcts, on the risk of dementia. This presentation will give a summary of the evidence as well as an update on the recent findings. Many questions remained unanswered and this talk will address some of them: Are some lesions more important than others on the risk of dementia? Do these lesions have the same risk factors and the same mechanisms? Are dilated perivascular spaces another example of small vessels disease? How efficient are antihypertensive drugs on the control of white matter lesions? 


\section{PS5: Translational Neuroscience: A Practical Approach}

\author{
PS5-1 \\ Silent Cerebrovascular Disease - An Emerging
Epidemic \\ Eprrving $B$. \\ Lund University, Sweden
}

That brain infarcts could be "silent" has been known for long from autopsy studies, but the magnitude and importance of silent cerebrovascular disease did not emerge until the introduction of neuroimaging techniques. "Silent" brain infarcts were first mainly studied in patients with acute stroke. During the 1990-ies several large studies using MRI in the healthy elderly population revealed surprisingly high prevalences of silent infarcts, which were detected in a quarter or more of persons older than 70 years. The majority of silent infarcts are small and subcortical, corresponding to small vessel disease. Autopsy studies have shown that the vascular lesions behind symptomatic and silent small deep infarcts are identical, it is only the localization that differs. The term "silent" should be understood in the sense that infarcts have not caused acute neurological deficits, and not in the sense that the brain lesions are innocent. Follow-up studies have shown that the presence of silent brain infarcts doubles the risk of developing cognitive decline and dementia and major vascular events like stroke. Studies have shown that silent brain infarcts are associated with major traditional risk factors in particular age and hypertension. Cerebral microbleeds were detected in 1996 through MRI using sequencies sensitive to hemosiderine products (gradientecho, or T2*). Several later studies have demonstrated that cerebral microbleeds are highly prevalent both in the general population as well as in patients with stroke. Detection rates increase with high-field MR techniques, which demonstrate a prevalence of up to $20 \%$ of cerebral microbleeds in the elderly general population. Rates are about twice in persons with acute ischemic stroke and about $3 \mathrm{x}$ higher among patients with intracerebral hemorrhage. Cerebral microbleeds increase with age, and are also strongly linked to hypertension. More recent studies have shown that deep cerebral microbleeds are associated with small vessel disease, whereas cortical microbleeds are associated with cerebral amyloid angiopathy. The field of silent brain infarcts and microbleeds is currently one of the major research areas in cerebrovascular diseases. Both enteties appear to interact with acute stroke pathophysiologies influencing the short and long term course of an acute stroke event. Several ongoing studies aim to delieneate to what degree cerebral microbleeds are associated with bleeding events during antithrombotic therapies including anticoagulants. Other studies aim to further delineate the course of the lesions. The finding that $>90 \%$ of all vascular injuries to the brain did not present as acute neurological dysfunction but consisted of "silent" brain infarcts and microbleeds have changed the concepts of cerebrovascular disease. Silent cerebrovascular disease is not benign, and with increasingly higher proportions of elderly per- sons in the population it constitutes a major public health issue. It is currently unknown to what extent silent cerebrovascular disease can be prevented or the course be modified, but general principles of risk factor/life style intervention on a population-wide basis is likely to be the most important approach.

\section{PS5-2 \\ Gene-Environment Interactions Mediating Experience-Dependent Plasticity in the Healthy and Diseased Brain}

Hannan A., Pang T., Renoir T., Burrows E., Ransome M., Mo C., Short A., DuX.

Florey Institute of Neuroscience and Mental Health, University of Melbourne, Australia

A major challenge in neuroscience involves understanding how specific genetic and environmental factors combine to predispose individuals to particular brain disorders. Harnessing such knowledge will facilitate targeted strategies for prevention and treatment. We have been investigating how various environmental manipulations selectively alter long-term gene expression, and associated cognitive processes and behaviours, in mouse models. Huntington's disease (HD) is a tandem repeat (CAG) expansion disorder involving a triad of psychiatric, cognitive and motor symptoms. In a transgenic mouse model of HD we have demonstrated that environmental enrichment (which enhances sensory stimulation, cognitive activity and physical exercise) can delay onset of the affective (depression-like), cognitive and motor endophenotypes. Environmental enrichment also induces cognitive enhancement in wild-type animals. Further studies have revealed that enhanced voluntary physical activity alone can have beneficial effects, although environmental enrichment exerts the greatest therapeutic influence, delaying onset and slowing progression. Environmental enrichment and wheel running induce a range of spatiotemporally distinct molecular and cellular changes associated with improved cognitive performance. Our results also suggest that the timing and duration of these environmental manipulations are critical in terms of their ability to selectively induce cellular plasticity and cognitive enhancement. The original discovery that environmental enrichment delays onset of disease in a genetic mouse model (van Dellen et al., 2000), has led to over a decade of follow-up studies in animal models of a range of different brain disorders (Pang and Hannan, 2012). For example, in knockout mice modelling autism-like and schizophrenia-like endophenotypes we have shown that environmental enrichment can also ameliorate cognitive and sensorimotor deficits. These findings have been extended to additional environmental factors (e.g. stress) and animal models of other disorders. Together with epidemiological findings and clinical trials, these studies are guiding the design of ongoing intervention studies for various neurological and psychiatric disorders. Furthermore, we are identifying molecular targets for 'enviromimetics' (Nithianantharajah and Hannan, 2006), a proposed class of drugs which could mimic or enhance the beneficial effects of complex mental activity and physical exercise. 


\section{PS6: Updates on Parkinson's Disease}

\section{PS6-1 \\ Initiating Therapy in Newly Diagnosed Parkinson's Disease}

Bharucha N.E., Lalkaka J.

Bombay Hospital Institute of Medical Sciences, India

Probably in no other neurodegenerative disease is there such a variety of treatment options available to the treating physician as in Parkinson's Disease (PD). Hence, initiating treatment of a newly diagnosed patient can be a challenging task Earlier, based on the fact that anti PD drugs give only symptomatic benefit, it was believed that PD drug therapy should be started only when motor symptoms interfered with the person's daily activities. No drug has been shown to have a definite neuroprotective effect. However, review of data from the ELLDOPA, DATATOP and TEMPO trials suggests that patients who were initially started on anti PD drugs continued to fare better over a prolonged period compared to those who were started on placebo. Therefore, even if symptoms are mild, most clinicians now start drug therapy as soon as PD is diagnosed. Levodopa is the most efficacious drug for PD, universally accepted as the "gold standard." However, its long term use leads to motor complications like "wearing off" and dyskinesias. Dopamine Agonists, in particular ropinirole and pramipexole, are also effective in controlling motor symptoms. Two randomized controlled trials comparing levodopa with ropinirole / pramipexole in PD patients found them to be nearly as efficacious as levodopa, with significantly less incidence of dyskinesias. Therefore use of pramipexole or ropinirole could help to delay the introduction of levodopa and enable a lower dose of levodopa to be used. The MAO B inhibitors, selegiline and rasagiline, also provide good symptomatic benefit both in early and advanced PD. Amantidine has weak anti PD efficacy and is generally considered unsuitable for monotherapy. Anticholinergic drugs may be used in some young PD patients with a significant rest tremor. They are are best avoided in the elderly because of their high incidence of cognitive disturbance, urinary retention and other side effects. The initial treatment in PD must be individualized keeping in mind the patients age, lifestyle, employment status, economic status and comorbidities. Levodopa would usually be the drug of choice in an elderly patient over the age of 70 years. In a young patient less than 50 years with mild to moderate motor impairment, it would be advisable to delay the use of levodopa and start on either a dopamine agonist or MAO B inhibitor. Between 50-70 years, the choice of drug would depend on the degree of motor disability. Dopamine agonist or MAO B inhibitor can be used if symptoms are mild. Significant motor impairment would necessitate levodopa as the initial drug. It is important to note that non motor symptoms of PD like depression, anxiety, constipation, olfactory disturbance and REM sleep behaviour disorders may be seen very early in the course of the disease and may, at times, even precede the onset of motor symptoms. They, too, significantly impact the quality of life of PD patients. It is therefore important to recognize these symptoms and treat them if they are present.

\section{PS6-2 \\ Parkinson's Disease Epidemiology Update}

Chen $\mathrm{H}$.

NIEHS, USA

Two years ago at this meeting, I presented data on smoking, urate, nonsteroidal anti-inflammatory drugs and exercise in relation to Parkinson's disease. In this presentation, I will discuss recent findings on other risk factors and on premotor symptoms. Several recent epidemiological studies have linked specific pesticides to Parkinson's disease. For example, farmers who used rotenone or paraquat had twice the risk for Parkinson's disease than nonusers. The recent finding on exposure to trichloroethylene and Parkinson's disease is also interesting, particularly given the ubiquitous presence of this organic contaminant. However, this analysis was based on very small numbers and awaiting for confirmation. Another notable finding is the potential link between cancer and Parkinson's disease; in particular, that Parkinson's patients are more likely to have melanoma and vice versa. Understanding this relationship may eventually shed light on common etiologies of these seemingly unrelated diseases. Finally, a recent study reported the first potential gene-environment interaction that was identified from genome-wide analysis and replication. In this paper, a genetic variant at the GRIN2A gene modifies the protective effect of caffeine on Parkinson's disease. Nonmotor symptoms are common among Parkinson's patients, and some may even precede Parkinson's motor onset by years. Theses "premotor" symptoms have recently attracted a lot of attention because they may offer a unique opportunity for identifying high risk populations and understanding early disease etiology. However, our current understanding of these premotor symptoms and their relationship to Parkinson's development is still in its infancy. Many fundamental questions are to be answered. For example, their temporal relationships to Parkinson's: when do they occur and how do they progress. As none of these premotor symptoms are specific to Parkinson's disease, the key is to identify combination(s) of these premotor symptoms that are highly predictive of Parkinson's disease and could also be easily assessed in large population based studies. Potential candidates include smell loss, REM sleep behavior disorders, and constipation. Until now, several fundamental questions on Parkinson's epidemiology remain unanswered, for example, what explain(s) the gender difference in Parkinson's incidence; are there any racial difference in Parkinson's disease, if so, why? Answers to these questions will lead to better understanding of Parkinson's etiology and disease prevention. 


\section{PS7: Epilepsy \& Neurodegenerative Disorders}

\section{PS7-1}

\section{The Long-Term Prognosis of Epilepsy}

BeghiE.

Mario Negri Institute for the Pharmacological Research, Italy

Epilepsy is a chronic treatable clinical condition characterized by repeated unprovoked seizures. In the first half of the 20th century, studies mostly done in referral centers reported seizure remission only in up to $42 \%$ of cases. Selection bias (the more severe cases being over-represented in clinical series) was a possible explanation of the fairly low relapse rates. In fact, when more representative patient cohorts (eg, newly diagnosed patients) were considered, the outcome of the disease was more favorable and remission of seizures was reported in up to $90 \%$ of cases. A satisfactory response to the initial treatment wasalso found to improve the early prognosis of the disease. However, later investigations, which included randomized clinical trials, failed to demonstrate an impact of early treatment (including treatment of the first seizure) on the long-term outcome of epilepsy. In more recent years, the long-term prognosis of epilepsy was assessed in small patient cohorts and different patterns were detected. In a Finnish population-based study of patients with onset of seizures as children and followed for an average period of 37 yearst (Sillampaa \& Schmidt, Brain 2006; 126:617-24) the course of the disease was predicted by the response to treatment during the early period after diagnosis only in some cases and five main patterns could be recognized: 1. Remission within the first year of treatment (early remission) continuing to terminal remission ( $16 \%$ of cases); 2 . Remission after the first year of treatment (late remission) continuing to terminal remission (32\%); 3. Relapsing-remitting course, ie remission following a relapse after an early or late remission (19\%); 4. Worsening course, ie no remission following a relapse after an early or late remission (14\%); 5. No remission ever (19\%). Other reports confirmed in part these findings casting doubts on the response to the initial treatment as a prognostic indicator and arguing in favor of the possibility that the response to antiepileptic drugs may be permanent, transient or null depending on some as yet unknown features pertaining to the patient and the assigned treatment. On this basis, it is still unknown whether epilepsy is a pre-determined clinical condition (with an at best modest effect of treatment on the long-term prognosis of the disease) or it is a dynamic process influenced by several factors, including treatment. These contrasting views can be addressed only with a large representative cohort of newly diagnosed patients followed for a prolonged period of time.

\section{PS7-2 \\ Essential Tremor and Its Associations with Other Neurodegenerative Diseases: What Is the Epidemiological Evidence?}

Louis E.

Columbia University, USA

Background: The possible links between essential tremor (ET) and Parkinson's disease (PD) and, more recently, between ET and Alzheimer's disease (AD) have been of growing interest, particularly with the emergence of data from postmortem studies suggesting that ET itself is likely to be a neurodegenerative disease. There are also some data suggesting a link between ET and progressive supranuclear palsy (PSP). Given the very high population prevalence of $\mathrm{ET}$, its possible role as a risk factor for $\mathrm{PD}$ and $\mathrm{AD}$ is of particular significance. Yet these data are controversial. At the center of this debate is the burgeoning evidence from epidemiological studies, examining whether there is an increased co-occurrence of these conditions within the same individuals or within families.

Methods: The author conducted a PubMed search from 1966 to September 2012 and reviewed the epidemiological evidence, generally restricting our analyses to studies that generated quantifiable measures of association (odds ratios or relative risks), thereby being able to assess the role of chance.

Results: The most robust evidence, from case-control, prospective and familial aggregation studies, indicates that ET is associated with increased odds and increased risks of both PD and $\mathrm{AD}$. In particular, a prospective, population-based study in Spain in 2010 reported that the risk of incident PD was more than four time higher in ET cases than age matched controls (relative risk = 4.27, $\mathrm{p}=0.002$ ). Two population-based, prospective studies (in New York and Spain) have demonstrated that the risk of incident dementia is elevated in ET cases compared to age-matched controls (relative risks $=1.64-1.98$, $\mathrm{p}$ values $0.01-0.055$ ). Initial data also support the increased odds of PSP in patients with ET in brain bank studies.

Conclusion(s): There is reasonable epidemiological evidence to support a link between ET and these neurodegenerative diseases. Further studies are needed to corroborate the current results, provide additional estimates of effect and begin to explore the mechanistic underpinnings for these intriguing associations. 


\section{PS7-3}

Updates on Epidemiology \& Management of Epilepsy

BeghiE.

Laboratory of Neurological Disorders, Institute for

Pharmacological Research "Mario Negri", Milano, Italy

Epilepsy is a chronic clinical disorder characterized by repeated unprovoked seizures affecting both sexes and all ages with worldwide distribution. Epidemiological studies on epilepsy must be interpreted in the light of methodological differences and the quality of the reports. Methodological constraints and inconsistencies are mostly prevalent in reports from developing countries, which face problems with case ascertainment and study conduct. The incidence of unprovoked seizures is 33-198 per 100, 000 and the incidence of epilepsy is $23-190$ per 100, 000. The rates in Europe and North America ranges from 24 and 53 per 100, 000 per year. The incidence varies with age (younger children and the elderly being at highest risk), etiology, seizure type, and epilepsy syndrome. The overall prevalence of epilepsy ranges from 2.7 to 41 per 1,000 population, but the rate for active epilepsy varies from 4 to 8 per 1, 000 . The prevalence of active epilepsy is generally lower in industrialized countries than in developing countries, which may reflect a lower prevalence of selected risk factors (mostly infections and traumas), a more stringent case verification, and the exclusion of provoked and unprovoked isolated seizures. As with incidence, the prevalence of epilepsy varies with age, etiology, seizure type, epilepsy syndrome, and socio-economic class. At least in part, the risk of epilepsy is accounted for by genetic factors. Genetic or chromosomal syndromes associated with epilepsy account for 2-3\% of all epilepsies, while syndromes characterized by simple mendelian inheritance or cytogenetic abnormalities are about 1\%. Epilepsies with complex inheritance include the majority of the epileptic syndromes reflecting a gene-environment interactions. Prenatal and perinatal factors are established environmental risk factors but with inconsistent findings, which indicate, at best, a moderate association. Mental retardation and cerebral palsy are the strongest risk factors for epilepsy. CNS infections are worldwide risk factors involving all ages. In developing countries, where infections diseases are commoner than in industrialized countries, epilepsy due to infections has higher prevalence in rural compared to urban areas. In these countries, mostly in South America, neurocysticercosis is a major cause of epilepsy, followed by cerebral malaria, tuberculosis and toxoplasmosis. Alzheimer's disease carries a sixfold risk of unprovoked seizures and other types of dementia an eightfold risk. Stroke and traumatic brain injury (TBI) are also implicated, with a direct correlation with disease/injury severity. Compared to ischemic stroke, hemorrhagic stroke (primarily subarachnoid hemorrhage) is followed by a higher risk of seizures. Traumatic events provoking concussion with no evidence of tissue disruption usually are not followed by epilepsy. In severe TBI the risk is highest during the first year and diminishes during the ensuing years. After 10 years, only severe injuries still exhibit an increased risk of seizures. Antiepileptic drugs (AED) and, in selected instances, resective surgery are the main therapeutic options. Despite the increasing number of drugs in the market, there is no evidence that AED modify the natural history of the disease. The number of drug-resistant epilepsies may be reduced by surgery but the long-terms effects of surgery are still scarcely understood.

\section{PS8: ICD-11: Implications for Neurology}

\author{
PS8-1 \\ Updates on Classification of Cerebrovascular \\ Diseases
}

Norrving $B$.

Lund University, Sweden

ICD-10 was created more than 20 years ago. Since then dramatic improvements in cerebrovascular diseases have taken place, with respect to pathophysiological knowledge, diagnostics, and therapies. In ICD-10 cerebrovascular diseases was spread out over several chapters (e.g., TIA appeared under nervous system disorders whereas stroke appeared under circulatory disorders). Several items were subject to frequent misclassifications in clinical practice. In ICD-11 all cerebrovascular disgnoses will form one single block under Diseases of the Nervous System, in line with current concepts of the disease. Furthermore ICD-11 will contain definitions of all items. Members of the Cerebrovascular Diseases working group are Bo Norrving (chair), Valery Feigin, Padma Gunarathne, Vladimir Hachinski, Michael Hennerici, Ming Liu, Peter Rothwell, Jeffrey Saver, In ICD-11 cerebral ischemic stroke is defined as "acute focal neurological dysfunction caused by focal infarction at single or multiple sites of the brain or retina". Evidence of acute infarction may come either from symptoms lasting $>24$ hours, or neuroimaging. Conversely, TIA is defined as an episode of acute neurological dysfunction without demonstration of brain tissue, and resolution of symptoms $<24$ hours. Hence, the definition of TIA will now be tissue based. The term "stroke" requires the presence of acute neurological symptoms. The section of "Other cerebrovascular disorders" has been thoroughly revised according to developments in pathophysiology. The term "Late effects of cerebrovascular disease" has been redifined to avoid misclassification of late effects vs acute stroke. Finally a section on "silent" infarcts and hemorrhages (microbleeds) have been added. Such diagnoses are not included in the diagnosis of "stroke" as they do not cause acute symptoms. The update of cerebrovascular diseases is probably the part in ICD-11 that has undergone most changes compared to ICD-10.

\section{PS8-2 \\ Should We Use Different Classifications for Different Purposes?}

BeghiE.

Laboratory of Neurological Disorders, Institute for Pharmacological Research "Mario Negri”, Milano, Italy

Disease classifications provide a common framework for clinicians and researchers. Practicing physicians should find in a classification a usable tool for recording clinical assessment and to communicate with other physicians. Researchers use the classifica- 
tion to distinguish different disease types in terms of incidence, prevalence and prognosis and to collect cases having similar features. Insurers and hospitals employ a classification to assess the burden of disease for the health system and the society and for assessing reimbursement. For all these uses, it is necessary for the classification to have good to excellent reliability across individuals. Instruments used for disease classification are subjected to changes over time as they must adapt to the advancing knowledge and the increasing number of diagnostic resources. The World Health Organization (WHO) has the responsibility of developing and updating the International Classification of Diseases (ICD), a standardized classification system to be used worldwide for morbidity and mortality statistics and by physicians, health care administrators and researchers working in health care facilities with differing resources and expertise. The most recent edition of the ICD codes, the ICD-10, was developed to provide an individual code for almost every neurological condition, so that a uniform classification could be available for epidemiological and clinical research as well as for routine statistical reporting. However, the results of studies testing the reliability of the ICD-10 and the antecedent ICD codes are inconsistent. The variability of the study findings, when used in differing contexts, speaks against the indiscriminate use of a classification to satisfy differing needs and, in this regard, perhaps different instruments should be devised ad used for practical, administrative, and research purposes. The reliability of the ICD codes and other classification systems will be critically appraised and exemplified by examining studies done in epilepsy.

\section{PS9: Rare Neurogenetic Disease: Practical Approach}

\section{PS9-1 \\ Niemann - Pick C Disease: Update on Epidemiology, Diagnostic Strategies and Management}

Vanier M.T.

INSERM and Hopitaux de Lyon, Lyon, France

Niemann-Pick disease type C (NP-C) is a rare neurovisceral lysosomal lipid storage disorder with autosomal recessive inheritance, caused by mutations on either of two genes, NPC1 and NPC2. The NPC1 and NPC2 proteins act cooperatively to facilitate the egress of free cholesterol from the endolysosomal system, but their complete function(s) have not yet been fully elucidated. The clinical spectrum is very wide. Except for $10 \%$ of the cases who die neonatally or before 6 months of age from a systemic disorder, the neurological involvement is what defines the severity of the disease. According to the age of onset of neurological symptoms, the patients are usually classified into early infantile, late infantile, juvenile and adolescent/ adult neurological forms. The neurological disorder consists mainly of cerebellar ataxia, dysarthria, dysphagia, and progressive dementia. Gelastic cataplexy, seizures, dystonia, and in later onset cases, psychiatric involvement and movement disorders, are also common. The most characteristic sign is vertical supranuclear gaze palsy. A disability scale evaluating ambulation, manipulation, language and swallowing has been proposed to calculate a severity score. Since 1975, nearly 200 patients have been diagnosed in France. The estimated incidence at diagnosis (1996-2011) appears close to 1 in 100000 live births, with an estimated prevalence in the order of 1 in 1 million. More patients with neurological onset in adolescent or adult age have been recognized in recent years, and in France, they constitute close to $40 \%$ of the currently alive patients. We observed a correlation between age of onset of the neurological disease and life span, but no correlation between the age of onset of the systemic disease and the neurological form. Laboratory diagnosis of NP-C is not straigthforward. The current gold standard approach ("filipin test") requires cultured fibroblasts from a skin biopsy, on which a lysosomal sequestration of cholesterol is demonstrated by fluorescence microscopy after staining with a probe called filipin. A biological variation ("variant" patients with little cholesterol accumulation in their cells) complicates the situation. Specialized laboratories are required for proper interpretation. Also gene sequencing has shortcomings (are variations disease-causing?). MLPA is often useful in case of unidentified mutations (deletions, duplications). Both cell biology and genetic tests are flawed, but they complement each other. Great hope is currently held on a new screening test studying cholesterol oxidation markers in plasma, which starts to be implemented in several clinical laboratories over the world. Proper symptomatic management of patients is essential. For specific management of neurological manifestations, a first product, miglustat, has now been approved in the EU and many other countries. Results from the clinical trial as well as from other published studies indicate that it may stabilize the disease, at least for a period of time, and that it appears to be more beneficial to later onset, more slowly progressive patients. Updated diagnostic and management guidelines have recently been published (Patterson et al. Mol Genet Metab (2012) May 7 E-pub ahead of print).

\section{PS9-3
Central Nervous System (CNS) Infections and Epilepsy \\ Bharucha N.E. \\ Bombay Hospital Institute of Medical Sciences, India}

The relationship between CNS infections and epilepsy is particularly important in low and middle income countries (LMIC) compared with high income countries (HIC). In Rochester Minnesota, CNS infections accounted for 3\% of newly diagnosed epilepsies, whereas in Sub-Saharan Africa this figure was $26 \%$. Conversely, CNS infections result in early onset seizures and chronic epilepsy in both HIC and LMIC, and their frequency depends on the nature of the underlying condition. For example, in Olmsted County Minnesota, epilepsy occurred 4.2 times more often than in general population if the underlying condition was bacterial meningitis, but 16.2 times more often if it were encepha- 
litis. Such data are unavailable in LMIC. Factors responsible for epilepsy following CNS infection are the patient's age, aetiology, acute symptomatic seizures, focal neurological deficits and parenchymal lesions, e.g. abscess, granuloma, infarct and encephalitis. I shall explore the contributions of neurotuberculosis, neurocysticercosis and cerebral malaria to seizures and epilepsy in LMIC. Problems in exploring these relationships include: 1) Difficulty in confirming the causative brain lesion, (e.g. tuberculoma vs cysticeral granuloma), due to lack of diagnostic facilities such as neuroimaging and EEG. Also, mere presence of a lesion does not imply causation of epilepsy. Calcified lesions are often found in the brain, in areas endemic for cysticercosis. In the instance of cerebral malaria, retinopathy is strongly correlated with brain lesions and hence its presence is used as a diagnostic marker. 2) It is often not possible to identify the causative organism in the brain. Even where laboratory tests are available (e.g. immunological tests for cysticercosis) they can at best establish systemic exposure, which may have occurred in the past. 3) A lack of analytic case control studies in LMIC. The few that have been carried out have looked at prevalent cases rather than new onset disease. 4) Studies in conditions such as neurocysticercosis need to ensure that they are dealing primarily with unprovoked rather than provoked seizures. Knowledge of the specific causative infection is important as it determines the course, prognosis, and treatment. Treatment usually involves tackling the underlying disease, the inflammatory response and the seizures. Antiepileptic drugs may have to be tailored to avoid interaction with drugs used in treating the underlying infection, e.g. Tuberculosis or HIV. Timely treatment in the acute phase may even influence the process of epileptogenesis and is advanced as a rationale for treating solitary cysticercal granuloma with albendazole and steroids.

\section{PS10: Methodological Issues in Neuroepidemiology}

\author{
PS10-1 \\ Neuroepidemiological Extension to STROBE \\ Brayne C. \\ University of Cambridge, United Kingdom
}

Although it does not receive as much funding as other areas of major health concern there has been substantial investment in neurological and neuropsychiatric conditions around the globe over the last decades. Most of the researchers working in these areas are keen to not only advance science but also for this science to have as much impact as possible through high level publications. Indeed investment in scientific careers and research in universities is often predicated on publication in top rank journals. This, with the promise of treatment and long term prevention through alteration of natural history at the molecular level, has resulted in most investment for neurology being directed towards these areas. The area of neuroepidemiology, despite being fundamental to our understanding of importance, policy and for insight into aetiology is relatively unattractive. Much work though in the clinical translation of the biomedical does contain information that could be capitalised for neuroepidemiology. However, these nuggets of evidence are often lost in the writing up of papers as they are not seen to be of sufficient relevance to the particular message chosen by the researchers. This is a shame since secondary researchers, with a population focus, could derive valuable information from many publications should the relevant information be there. Clear standards for reporting the populations from which clinical samples are derived, the nature of the health services through which patients are seen and the way these studied groups relate to their original populations are critical pieces of information. The ongoing exercise in the Global Burden of Diseases has highlighted these deficiencies in that very few studies have sufficient quality reporting to contribute fully to the exercise. This limits the range of papers included, and often leads to the exclusion of the very small number of studies available in certain parts of the world. This presentation will discuss these issues and describe current attempts to create a movement to improve this for neuroepidemiology.

\section{PS10-2 \\ Innovative Methods for Neuroepidemiologists}

Nelson L.

Stanford University School of Medicine, USA

Introduction: Neuroepidemiology has evolved into a discipline that is central to the study of neurological disorders, both in the clinic and in the population. In the current funding era, however, neuroepidemiologists are faced with dwindling resources and the need to propose research projects that have innovative appeal. I will discuss innovative methods neuroepidemiologists can use in two contexts: (i) using data mining approaches for classification and prediction, and (ii) translating basic neuroscience discoveries to improve the neurological health of patients and populations. Use of Data Mining Approaches. Innovative data analysis approaches may yield new research insights when applied to the large amount of data collected in neuroepidemiology studies. I will present two examples from my own research that illustrate the utility of data mining techniques: (i) data mining methods as adjuncts to classical statistical methods for identifying high risk subgroups in case-control studies, and (ii) data mining methods used with large health care utilization databases to identify patients with neurological disorders with a high degree of accuracy. Translating Basic Neuroscience Discoveries. Epidemiology is increasingly recognized as an important component of translational research, especially for translating basic scientific discoveries into new methods of diagnosing, treating, and preventing neurological disorders. I will present examples of the central importance of neuroepidemiology in all phases of translational research (T1 through T4). Summary. In the United States, funding for comparative effectiveness research (translational research at the T3, T4 levels) has dramatically increased in recent years. In a 
recent Institute of Medicine report that highlighted research priorities for comparative effectiveness research, common neurologic disorders (e.g., stroke, headache, multiple sclerosis, epilepsy, and dementia) were identified as areas of research priority. Neuroepidemiologists can play a central role in translating basic research discoveries into approaches that can benefit patients in the clinic and in the population. Innovative methods such as data mining approaches can lead to research advances in neuroepidemiology and improve the chances for receiving funding at a time when funding is near a historical low.

\section{PS11: Updates on Multiple Sclerosis}

\section{PS11-1}

\section{Status of Multiple Sclerosis in Developing Countries}

Pandian J.D.

Christian Medical College and Hospital, India

Multiple Sclerosis (MS) is a progressive inflammatory, demyelinating neurological disorder with a multifactorial origin that varies in presentation, geographical location, timescale of symptoms and severity. MS is relatively a rare disease in the developing countries. This presentation is aimed to assess the epidemiology, pattern, treatment options and issues in the management of MS in developing countries. The availability of data is limited due to the low prevalence of the disease in the Asian and African continents, the long-time interval between the first symptom and the establishment of the diagnosis, and the lack of diagnostic facilities to confirm the disease. MS is more prevalent in developed nations with the highest prevalence being reported from most of Europe, Israel, Canada, northern USA, south-eastern Australia, and New Zealand with a prevalence ranging between 50 - 100/ 100, 000 individuals. In the developing countries, the epidemiological burden of MS is noted to be the highest in Eastern Europe $(0.3-$ 205/100, 000), followed by South America from Panama to Argentina $(0.75$ - 21.5/100, 000) and Asia (0.7 - 13/100, 000) including Middle East $(0.4-101 / 100,000)$. MS is not well documented in Africa. In India, the overall prevalence as expected was on the lower spectrum $(<5 / 100,000)$ However, the Parsi community in India showed a prevalence of 26 per 100, 000 individuals. The pattern of the disease is also different in developing countries particularly in Asia where optico-spinal MS form is common. The infrastructure required for the diagnosis of MS in developing countries is also variable and scarce. MRI scanners are available in bigger cities and in major public and private hospitals. Moreover, the number of neurologists in these countries is also less. The drugs used to treat MS is also expensive in developing countries and very few are able to afford the treatment. The post MS care particularly rehabilitation is also not available in all hospitals. Diagnostic practices and treatment of MS in developing countries appear to lag behind those in developed countries and there is lack of public and professional awareness towards MS. There is need for adequate education, advice, support and awareness towards coping with MS and also a need for increasing the availability of disease modifying treatments. Future goals involve making health services and rehabilitation facilities available for MS patients and develop MS societies and support groups in the developing countries.

\section{PS11-2 \\ What Causes Multiple Sclerosis?}

Taylor Bruce

Menzies Research Institute Tasmania University of Tasmania, Australia

Multiple sclerosis (MS) is the most common cause of nontraumatic neurological dysfunction in young adults of European ancestry. Despite nearly a century of intensive study the exact cause of MS has not been fully elucidated. What is clear is that there is no single factor that results in an individual developing MS, rather a combination of factors acting at different time points are required for the manifestation of clinical disease. The factors that are recognised as predisposing a person to developing MS include: 1. environmental factors particularly low vitamin D levels/ultraviolet radiation exposure manifesting as the latitudinal gradient of MS prevalence, lower numbers of pregnancies in females, lower exposure to infant siblings (the hygiene hypothesis) and increased levels of smoking. 2. Genetic factors, particularly SNPs in genes of the immunological system. 3. Exposure to the Epstein Barr Virus in adolescence and exposure to the HHV6 virus. How these factors interact to produce MS and the timing of these interactions appear to be very critical in starting a person on a cascade that leads to clinical MS. This talk will present the current status of research into the causes of MS with results from the Australian Multicentre Study of Environment and Immune Function (AUSIMMUNE Study), the Tasmanian MS longitudinal study, The Australian \& New Zealand MS genetics Consortium Study (ANZGENE), and The New Zealand MS Prevalence Study (NZMSPS). Comprising 4 major epidemiological and genetic studies of MS that have provided important insights into the cause of MS and have allowed analysis of how the established risk factors interact with genetic markers of MS risk and when these factors have their effects during development. 


\section{PS11-3}

The Origin and Spread of Multiple Sclerosis in Europe

Kurtzke J.

Georgetown University, USA

There are now some 500 prevalence or incidence studies of multiple sclerosis throughout the world, indicating that MS is a place-related disease. Migration studies further show that this is environmental and acquired in high risk areas. Earliest distributions found that all high risk areas were in northern Europe or its colonies. In the United States, draftees rejected for military service in World War I had significantly highest rates in the north central states of Wisconsin and Minnesota, which became states in 1848 and 1858. They were settled primarily by immigrants from Norway and Sweden, who came mostly from the south-central parts of each country, the regions of both lands that had been shown by nationwide prevalence studies before World War II to have the highest frequencies of MS in each country. In Fennoscandia it is likely MS spread from the Swedish focus eastward to Finland and westward to Norway. First spread on to the continent may have been by occupation of what is now central Germany by the armies of Gustav Adolph in the 30 Years War of 1618-1648. Swedish occupation sites were similar to high frequency regions for MS autopsies in Germany 1906-1950, and were closest to the highest MS regions in France in 1986 (northeast) and in Switzerland in 1922 (north central cantons). Swedish occupations later in the war in coastal Pomerania may have been the source of MS in Denmark, from its distribution 1921-1933. Continued diffusion has almost obliterated this north-south gradient of MS in Europe, with now spread into the Near East as well.

\section{PS12: Controversies in the Management of Neurological Disorders}

PS12-3

Neurorehabilitation - Applied Neuroplasticity Kesselring J.

Kliniken Valens, Switzerland

Re-organisation of structures and functions in the brain are the basis of learning. Plastic changes occur in normal as well as in diseased brains and can be enhanced by task-specific therapeutic interventions (Neurorehabilitation). Due to the variety of symptoms and functional deficits Multiple Sclerosis (MS) can lead to a broad range of functional impairments and handicap. Even with newer immunomudulating therapies, the course remains progressive. The symptoms themselves, loss of independence and participation in social activities are responsible for the progressive decline of quality of life. The main objective of a comprehensive rehabilitation program is to ease the burden of disease by improving self performance and independence. Restoration of function is not the key effect of rehabilitation in MS. As rehabilitation measures have no direct influence on the ongoing disease process and progression of the disease, compensation of functional deficits, adaptation and reconditioning together with other nonspecific effects (management of specific symptoms and impairments, emotional coping, self estimation) is more important in the longterm. Several of the many symptoms of MS are amenable to drug therapies which have been proven in careful evidence-based analyses to be effective (e.g. fatigue, spasticity, bladder, bowel and sexual disturbances, pain, cognitive dysfunctions etc). Newer studies in MS patients show, that despite the ongoing progression of the disease process, rehabilitation is effective by improving personal activities and participation in social activities leading to better quality of life. After comprehensive inpatient rehabilitation, improvement overlasts the treatment period for several months. Quality of life is correlated more with disability and handicap rather than with functional deficits and progression of the disease. Kesselring J, Comi G, Thompson AJ Multiple Sclerosis - functional recovery and neurorehabilitation Cambridge University Press 2010 Serafin Beer, Fary Khan, Jürg Kesselring Rehabilitation interventions in Multiple Sclerosis An overview J Neurol 2012 DOI 10.1007/s00415-012-6577-4 Mostert S, Kesselring J Effect of pulsed magnetic resonance therapy on the level of fatigue in patients with multiple sclerosis - a randomised controlled trial Multiple Sclerosis 2005; 11: 302-305 Kesselring J, Beer S Symptomatic therapy and Neurorehabilitation in multiple sclerosis The Lancet Neurology 2005; 4 (10); 643-652 Meyer-Heim A, Rothmaier M, Weder M, Kool J, Kesselring J Advanced cooling garment technology: functional improvements in thermosensitive patients with Multiple Sclerosis 2006; 12: 1 - 6 Multiple Sclerosis Therapy Consensus Group (MSTCG) of the German Multiple Sclerosis Society Symptomatic Treatment of Multiple Sclerosis Eur Neurol 2006; 56: 78 - 105 Kesselring J, Coenen M, Cieza A, Thompson A, Kostanjsek N, Stucki G Developing the ICF Core Sets for Multiple Sclerosis to specify functioning Multiple Sclerosis 2008;14: 252-4 Holper, Coenen M, Weise A, Stucki G, Cieza A, Kesselring J Characterizing functioning in MS using the ICF J Neurol 2010; 257: 103 - 113 Stützer P, Kesselring J Wilhelm Uhthoff - a phenomenon Int MS J 2008; 15; 90 - 93 Beer S, Manoglou D, Aschbacher B, Kool J Kesselring J Robot-assisted gait training in MS - a randomised controlled trial Multiple Sclerosis 2008; 14: $231-236$. 


\section{PS13: Alternative Medicine and Rehabilitation In Neurological Disorders}

PS13-3

\section{A New Model of Rehabilitation Study at Community Level for Stroke Survivors in Five Urban Populations in China}

Wang W., Ru X., Dai H., Wu S., Jiang B.

Beijing Neurosurgical Institute, China

Background and Aims: In China, more and more people survive strokes and the majority have to cope with the physical, psychological, social and functional sequelae. Due to limited rehabilitation resources, most patients have not opportunity to receive rehabilitation therapy. The purpose of this study is to explore and develop an effective and appropriate communitybased rehabilitation technology in China. Methods: A multi-center intervention study was conducted in five sub-centers of three cities in China. The intervention measures comprised a rehabilitation information package and a new rehabilitation exercise program that is a simplified form of Conductive Education. We randomly evaluated the awareness of rehabilitation and situation of receiving rehabilitation services in the stroke survivors. We used the Fugl-Meyer Assessment (FIM) to evaluate motor function, and Barthel index (BI) to evaluate activities of daily living (ADL).

Results: After intervention, the ratio of patients who had received rehabilitation education was from $37 \%$ to $61.7 \%$ in the intervention communities ( $\mathrm{p}<0.001$ ), $33.6 \%$ to $39.2 \%$ in the control communities $(\mathrm{p}=0.066)$. Three hundred and forty-two patients successfully completed three-month rehabilitation training in the community health neighbourhood service center. Before intervention, median score of FIM and BI were 46 (IQR 56) and 80 (IQR 40), and after three-month intervention, they enhanced to 62(IQR 54) and 85(IQR 40), respectively. There was significant difference between pre-intervention and post-intervention in FIM $(p<0.001)$ and $B I(p=0.008)$ score in this study group.

Conclusions: This new model of rehabilitation exercise program was proved economical, convenient, efficient on improving patients' motor function and ADL, and it is feasible in most urban communities in China.

\section{SS1: Satellite Symposium of the WFN "What Is an Environmental Factor?"}

\author{
SS-3 \\ Nodding Syndrome \\ Spencer $P$. \\ Oregon Health \& Science University, USA
}

Involuntary head nodding (wamesinzia kichwa; Swahili) in children who later developed grand mal seizures (kichwa, epilepsy) was first described in the 1960 s by Louise Jilek-Aall among the Wapogoro Tribe who live in the Mehenge mountains of Tanganyika (now Tanzania), with the earliest recorded onset dating from the 1930s (L. Jilek-Aall, unpublished data). Thirty years later, Gerrits noted that the Bassa and Kpelle people of rural Grand Bassa County, Liberia, distinguish between two types of epilepsy (sii): "to drop the head in the pan" and "the big jerking", the former showing similarities with absences or petit mal in western children. Nodding of the head, staring and automatisms (chewing, verbal, fumbling) were also independently and contemporaneously described in Bassa and Kpelle children prior to the onset of generalized tonic-clonic convulsions. In 2002, a short-term study by an interdisciplinary team organized by the World Health Organization described head nodding and convulsions in Moru but not Dinka children in Mundri County, Western Equatoria in today's South Sudan. At a similar time, head nodding in children was reported in northern Uganda where those affected now number in the thousands. This seizure disorder apparently has an environmental etiology but the causative factors are unknown. The condition typically arises in impoverished communities living near rivers and affected by displacement, poor nutrition, newly introduced plant foods, and onchocerciasis without blindness. Children with nodding syndrome are often stunted physically and mentally, withdrawn and stigmatized. Temperature, time of day and especially the act of eating local food characteristically trigger rhythmic head nodding or convulsions. In sum, this apparently progressive and fatal seizure disorder appears to have a complex environmental etiology that may involve geographic location, systemic infection (Onchocerca volvulus), nutrition and other factors. 


\section{Session 01: Prevention}

60

\section{Increased Risk of Stroke in Patients with Isolated Vertigo or Dizziness: A Retrospective Case-Control Study}

Barber P.A. ${ }^{a}$, Venkatesh K. ${ }^{a}$, Pridmore H. ${ }^{b}$, Roberts S. ${ }^{b}$, Carter K. ${ }^{c}$, Anderson N.E. ${ }^{\mathrm{b}}$

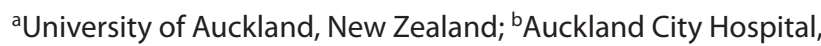
New Zealand; ' University of Otago, New Zealand

Background: Cannabis is the most widely used illicit drug of abuse. Case reports and case series have reported ischemic stroke and transient ischemic attack (TIA) developing during or within hours of cannabis use. However, to date there have been no prospective studies exploring a possible causal link between cannabis use and stroke. We performed a case-controlled study on the use of cannabis in younger stroke patients to investigate this link.

Methods: Consecutive stroke patients aged 18-55 years who had urine screens for cannabis between January 2009 and April 2012 were compared to a control cohort of age, sex and ethnicity matched patients admitted to hospital with non-cardiovascular diagnoses.

Results: One hundred and sixty of $218(73 \%)$ ischemic stroke or TIA patients aged 18-55 admitted to our service between January 2009 and April 2012, had urine drug screens [100 men, 60 women; mean (SD) age 44.8 (8.7) years], of whom 150 (94\%) had ischemic stroke and $10(6 \%)$ had TIA. Twenty-five of $160(15.6 \%)$ patients had cannabis detected in urine drug screens. Patients with positive cannabis screens were more likely to be male $(84 \%$ versus 59\%, $\chi^{2} \mathrm{p}=0.016$ ) and tobacco smokers (96\% versus $51 \%$, $\left.\chi^{2} \mathrm{p}<0.001\right)$. Control urine samples were obtained from 160 patients [mean (SD) age 44.8 (8.5) years]. The cases and controls were well matched for age (student's t-test, $\mathrm{p}=0.492)$, $\operatorname{sex}\left(\chi^{2} \mathrm{p}=\right.$ $0.471)$ and ethnicity $(\chi 2 \mathrm{p}=0.910)$. Thirteen of $160(8.1 \%)$ control participants tested positive for cannabis which was just over half that of the 25 stroke patients (odds ratio 2.09, 95\% confidence intervals $1.03-4.25, \mathrm{p}=0.019$ ).

Conclusion: Younger ischemic stroke and TIA patients were twice as likely as control participants to have recently used cannabis. Cannabis is generally perceived as having few serious adverse effects. This study suggests that this may not be the case and that the association between cannabis and stroke warrants further investigation.

\section{1}

\section{Endarterectomy Versus Angioplasty in Patients with Symptomatic Severe Carotid Stenosis (EVA-3S) Trial: Long-Term Results from a Randomized, Multicentre Trial}

Mas J.-L.

Centre Hospitalier Sainte-Anne, Paris, France

Background: Whether carotid stenting is as safe as carotid surgery and whether it provides long-term protection against recurrent ipsilateral stroke that is similar to that of surgery are unclear. We previously reported that in patients in the EVA-3S trial, the rate of any stroke or death within 30 days after the procedure was higher with stenting than with surgery. However, after the 30-day period, the risk of ipsilateral stroke was low and similar in both treatment groups, suggesting that carotid stenting is as effective as carotid surgery for medium-term prevention of ipsilateral stroke, at least for the first few years after the perioperative period. We now report on long-term follow-up of patients included in this study.

Methods: EVA-3S is a multicentre (30 centres in France), randomised, open, assessor-blinded, non-inferiority trial of stenting versus endarterectomy in 527 patients with carotid stenosis of at least $60 \%$ that had recently become symptomatic. This trial is registered with ClinicalTrials.gov, number NCT00190398. Patients were included between November, 2000 and September 2005 and the present study was conducted between December 1, 2011 and May 31, 2012. Vital status was ascertained through municipal death registries. Survivors at the time of the follow-up study were asked to participate in a structured interview. The interview schedule included questions aimed at detecting a new stroke, TIA, acute coronary syndrome, or endovascular or surgical revascularization procedure, including carotid, coronary or peripheral procedures. The modified Rankin Scale was assessed during the interview. Patients were asked to have carotid ultrasound performed and reports of most recent carotid examinations were collected. For patients who had died, physicians who had been in charge of the patients were contacted to obtain information about occurrence of outcome events (see above) before death and the cause of death. For patients who had died or were suspected of having had a recurrent stroke or other vascular event, all of the available clinical information and results of investigations obtained from records held by hospitals and physicians in private practice were independently reviewed and adjudicated by two physicians, blinded to treatment assignments.

Results and Conclusions: will be presented during the conference.

\section{KARGER \\ () 2012 S. Karger AG, Basel}

Fax +41613061234

E-Mail karger@karger.ch

www.karger.com
Accessible online at: www.karger.com/ned 


\section{7}

\section{Neuroprotective Effects of Palm Vitamin E Tocotrienols in Brain White Matter Lesion: Evidence from a Double Blind Placebo-Controlled Clinical Trial}

Gopalan Y.a, Magosso E. ${ }^{\mathrm{b}}$, Shuaib I.L. ${ }^{\mathrm{b}}$, Ansari M.A. ${ }^{\mathrm{b}}$, Abu Bakar M.R. ${ }^{b}$, Khan Nurzalina A.K. ${ }^{b}$, Wong Jia W. ${ }^{c}, \mathrm{Ng}$ Bee H. ${ }^{c}$, Nesaretnam K. ${ }^{\mathrm{d}}$, Sundram K. ${ }^{\mathrm{e}}$, Chinna K. ${ }^{\dagger}$, Kah Hay $Y^{\mathrm{b}}{ }^{\mathrm{b}}$

anniversity Technology Mara, Malaysia; ' University Sains

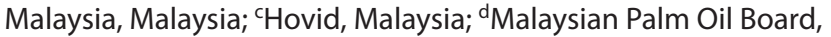
Malaysia; ${ }^{\mathrm{e}}$ Malaysian Palm Oil Council, Malaysia; ${ }^{\mathrm{f} U n i v e r s i t y}$ Malaya, Malaysia

Background: Cerebral white matter lesions (WMLs) are regarded as manifestations of small vessel disease which indicates subclinical vascular events and fragile brain vascular network. This study aimed to assess the development of WMLs among healthy volunteers treated with either $200 \mathrm{mg}$ tocotrienols softgels twice daily or a placebo for two years.

Methods: Upon screening 1300 healthy volunteers above the age of 35 with mild hypercholesterolemic condition from Malaysia, 390 subjects underwent brain imaging procedures using GE, Signa HDx 1.5 Tesla MRI scanner. Fasting blood samples were collected for full blood chemistry investigation. Brain imaging of WMLs using MRI were performed for baseline and repeated after 1 year and 2 years. The WMLs volumetric measurements were quantified using an extensible medical image analysis, visualization and annotation platform (Endeavor) software. Data were analyzed using SPSS software version 16.

Results: Based on the intention to treat analysis, MRI brain images of 121 volunteers were analysed, 62 in the treatment group and 59 in placebo. At the end of 2 years, we observed progression of the lesion in the placebo group [ $(270 \pm 730 \mathrm{ml}),(95 \% \mathrm{CI} 80$, $461), p=0.006$, effect size $(E S)=0.4$ ] which was not perceived in the treatment group $[(-46.1 \pm 721 \mathrm{ml}),(95 \% \mathrm{CI}-229,137), \mathrm{p}=$ 0.616 , effect size $(E S)=0.07]$. No clinically significant differences were found in terms of blood pressure, cholesterol, fasting blood glucose and body mass index ( $\mathrm{p}>0.05)$ between the two groups.

Conclusion(s): Subjects treated with tocotrienols showed volume regression of WMLs whereas significance increase was observed in the placebo group. Thus, tocotrienols exhibit neuroprotective effects to the brain from WMLs.

\section{5}

\section{Community Based Intervention Study on Improving Residents with Stroke-Related Knowledge in Four Cities in China}

Sun H., Wang W., Jiang B., Ru X., Wu S.

Beijing Neurosurgical Institute, China

Background: Effective stroke primary prevention depends on the level of knowledge of stroke in the general population. The study aim to assess the effect of intervention measures on community residents with stroke-related knowledge.
Methods: Select two communities mixed of social class and geography form Beijing, Shanghai, Chengdu and Changsha respectively. Divide randomly two communities into an intervention and a control community. From 2008 to 2010, comprehensive intervention measures, including public lectures and health educational materials to community residents and professional lectures to community medical staffs, were regularly implemented according to the study process in intervention communities. The awareness of knowledge about stroke among community residents were investigated before and after the comprehensive intervention. Every correct answer is recorded as one score (total score $=22$ ). Compared with control communities, evaluate the effect of intervention measures. This study was supported by the National Eleven-Fifth Scientific Research Project (Grant No. 2007BAI24B03).

Results: On the baseline investigation, there were no significant differences in residents' awareness of stroke risk factors and symptoms and total stroke knowledge scores between intervention and control communitiesi1/4 ${ }^{\wedge} \mathrm{P}=0.583 \mathrm{i} 1 / 4 \%$. After the comprehensive intervention, measures, the awareness of stroke risk factors and symptoms among intervention community residents were significant higher than those among control community residents $(\mathrm{P}<0.001)$. Total stroke knowledge scores of intervention community residents were significant higher than those of control community residents $(\mathrm{P}<0.001)$.

Conclusion(s): Comprehensive intervention measures can significantly improve cognitive level of knowledge about stroke among community residents.

\section{9}

Carotid Artery Stenting: Is There an Operator Effect? a Pooled Analysis of Individual Patient Data from Three Randomised Trials in the Carotid Stenting Trialists $^{\text {'TM }}$ Collaboration (CSTC)

Calvet D.

Centre de Psychiatrie et Neurosciences INSERM UMR 894, Paris, France

Background: At present, randomized clinical trials in patients with symptomatic carotid disease show inferior results of stenting compared to surgery with regard to the risk of stroke or death within 30 days of treatment. To improve the risk-benefit profile of stenting, it is crucial to establish which factors among patient characteristics and the procedure itself are associated with a high risk of stroke after carotid stenting. In this respect, we examined whether operator experience is associated with the 30-day risk of periprocedural stroke or death.

Methods: We pooled individual patient data from the three large European randomized trials of stenting versus endarterectomy for symptomatic carotid stenosis (Endarterectomy vs Angioplasty in patients with Symptomatic Severe Carotid Stenosis trial (EVA-3S), the Stent-Protected Angioplasty versus Carotid Endarterectomy trial (SPACE), and the International Carotid Stenting Study (ICSS). The analysis was done per-protocol, and included 1679 patients treated by CAS. The main outcome event was the occurrence of any stroke or death within 30 days of the 
procedure. We assessed the role of previous experience of operators before their participation in the trials, as well as the number of procedures performed by each operator during the trials. We adjusted for known risk factors for stroke during stenting.

Results and Conclusion(s): Will be presented during the conference.

\section{3}

\section{How to Increase the Impact of Trials Regarding CEA and CAS in Asymtomatic Carotid Stenosis?}

\section{Bartko D. ${ }^{a}$, Danihel L. a , Gombosova Z. ${ }^{a}$, Combor l. ${ }^{a}$, Blazicek P. , Fabcin J. ${ }^{a}$, Kalenska D. ${ }^{\mathrm{a}}$, Labanczova L. ${ }^{\mathrm{a}}$, Rusnak F. ${ }^{\mathrm{a}}$, Kurva E. ${ }^{\mathrm{c}}$, Nosal V.c \\ ${ }^{a}$ Central Military University Hospital, Slovakia; ${ }^{b}$ Alpha Medical Company, Slovakia; ' University Hospital Martin, Slovakia}

Background: Management of carotid diasease has changed many times over the last 50 yrs in response to high- level evidence from randomized controlled trials, technological inovations, improvements in RFs management and medical prevention. Optimal strategies to identify individuals for different procedures (CA, CAS, medical prevention) in asymptomatic pts remain broadly debated.

Aim: to propose new insights, strategy for decision regarding the optimal treatment strategy for pts with asymptomatic carotid stenosis (ACS) with particularly high risk for stroke(iCI).

Methods: Data from Cochrane, EMBASE, SCI, Trials (1993$2012)$, our results $(\mathrm{CEA}=358, \mathrm{CAS}=165)$, "leader expert's opinions" (2010-2012).

Results: Both procedures are performed: 1.at the site of carotid plaque, 2.baroreceptors/their sensitivity(BRS), 3.changing arterial wall(stiffnes, intima media thickness (IMT). BRS is very important factor involved in the compensatory hemodynamic mechanisms of the iCI onset, time-course.BRS closely controls heart rate and short-term changes in BP Therefore BRSmonitoring and $\mathrm{BP}+\mathrm{ECG}$ (simultaneously) is important factor influencing not only success of procedures but also outcome and prognosis.

Changes in stiffness, IMT and plaque can influence BRS, consequently compensatory mechanisms (e.g. BP, HRV), consequently perfusion blood pressure (PP) and $\mathrm{CBF}$ Therefore monitoring of all these parameters is importanti Stiffness and IMT represent a form of diffuse atherosclerosis whereas plaque is a surrogate for focal atherosclerotic process (stabile, unstabile and ulcerated). All parameters can be monitored and quantitatively evaluated. New specific inflammatory vascular enzyme LP-PLA2 (PLAC) brings new insights in understanding atherosclerosis and decision for procedures. It is not only a key inflammatory marker but it is also marker which is specifically linked to plaque inflammation an plaque ruptur It is highly specfic for both, vascular inflammation and plaque rupture. it can represent the novel thrapeutic target for blocking or reducing PLAC activity and slowing (or stoping) atherosclerotic plaque formation and plaque rupture.

Conclusion(s): Plaque ulcers and microembolization are associated with a high risk for stroke. Such pts should be considered for revascularization whereas pts without these findings should be managed medically. In these cases, complication rate is extremly low $(035 \%)$ (in this section, importance of results of the study for practice and/or future research is briefly described).

\section{7 \\ New Era In Understanding Atheroaclerosis and Plaque Morphology. From Cholesterol through Lipoproteins, LDL/HDL-Subfractions to Specific Vascular Inflammatory PLAC Marker}

Bartko D.

Central Military University Hospital, Slovakia

Background: For many years cholesterol and LDL-lipoproteins have been considered as the important factors in the development of atherosclerosis. Some years ago new data have been published, showing that specific vascular inflammation marker could be responsible for this mechanism.

Controversies Aim: To analyse various parameters of arterial wall (stiffness, IMT, plaque morphology) and PLAC enzyme in:1. ischemic cerebral stroke (iCI), 2. arterial hypertension (AH), coronary artery disease (CAD) and 4. controls (C). Prospective multicenter, multidisciplinary study.

Methods: Material consists of 766 subjects divided into four groups:1.ischemic Cerebral Stroke $(\mathrm{n}=127)$, mean age74.4+/-9.1, men $54.2 \%, 2$.Arterial Hypertension $(n=142)$, mean age $62.0+/-$ 12.2, men 48.3\%, 3.Carotid Artery Disease $(\mathrm{n}=120)$, mean age $71.8+/-1.8$, men $51.5 \%$, 4.Controls $(\mathrm{n}=126)$, mean age $48.5+/-$ 18.5 , men $52,7 \%$, without history of major CV RFs.

In all pts and controls, it was performed:NIHSS, BI, mRS, Lymphocyte-associated phospolipase A2(PLAC), Dia Dexus Inc. USA), lipid spectrum, hsTroponine, hsHcy, other biochemicalhematological parameters, CT/MRI(ischemic volume, a x b x c x 0.5), Stiffness, Intima media thickness-IMT(Sphygmocor, AtCor Sydney, USG), plaque morphology, long-term ECG and BPmonitoring simultaneously. Statistics:Student-test, PearsonSpearman-Kandell, Whitney U test.

Results: Development of atheroslerosis started by t-cholesterol, continued by lipopreteins, LDL/HDLsubfractions and at present new specific inflammatory vascular enzyme PLAC) is on horizon. Our study showed significantly higher activity of PLAC in. $\mathrm{AH}(219.01+/-27.64), \mathrm{iCAD}(234.02+/-51.58)$ and iCI groups (259.2+/-86) comparing to controls $(183,27+/-25.2)$. Close correlation between Tchol and LDL, was found, but no significant correlation between PLAC and T-chol. Stiffness, IMT were significantly higher in all followed groups. Significant correlatation between plaque morphology(ulcer), and PLAC was documented.

Conclusion(s): Study showed significant higher activity of PLAC, higher value of stifness, IMTand plaque morphology not only in $\mathrm{iCI} / \mathrm{CAD}$ but also in $\mathrm{AH}$ (quasi candidates of iCI) comparing to controls, It could signalized new era in understanding mechanisms of atherosclerosis and its complications. PLAC is a key inflammatory marker, specifically linked to plaque inflammation and plaque rupture. 


\section{Session 02: Diagnosis}

\section{1 \\ Novel Noninvasive Assessment of Functional Cerebral Artery Stenosis Computed from Computed Tomography Angiography}

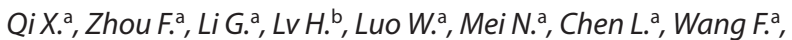
Xiao J.', Yang C. ${ }^{\mathrm{a}}$

aTongji Hospital, Tongji University School of Medicine, China; ${ }^{\mathrm{b}}$ Division of Mathematics, Tongji University, China; 'School of Life Science, Shanghai University, China

Background: Transstenotic pressure gradient (TSPG) and fractional flow reserve (FFR) are two well-evaluated functional index to assess the ischemic severity of coronary and renal artery stenosis, which has been reported to be a promising tool to identify the patients who would likely benefit from revascularization. This study aims to transplant the concept to cerebral artery stenosis and develop a novel noninvasive TSPG (TSPGni) and FFR (FFRni) assessment.

Methods: Cerebral TSPGni and FFRni were calculated through three dimensional modeling reconstructed from cerebral artery computed tomography angiography (CTA), finite element analysis and computational fluid dynamics. Clinical data were adopted and calculated as a pilot study to test the feasibility and accuracy of cerebral TSPGni and FFRni with diagnosis of color Doppler ultrasound (CDUS) and cerebral CTA as reference standards.

Results: Cerebral TSPGni and FFRni assessment were applied successfully in pilot study. The peak systolic velocity of cerebral artery of a moderate (left) and a mild (right) cerebral stenosis were 117 and $92 \mathrm{~cm} / \mathrm{s}$. The CTA images indicated that the degree of cerebral arteries stenosis was quantified as 60\% (left) and 15\% (right). Cerebral TSPGni were 59.2 (left), 31.6 (right) $\mathrm{mmHg}$ and FFRni were 0.22 (left), 0.43 (right), respectively.

Conclusion(s): The results of cerebral TSPGni and FFRni were generally consistent with the diagnosis of CDUS and CTA. Cerebral TSPGni and FFRni have been made up deficiencies in the field of evaluating functional cerebral artery ischemia. It is believed that the novel cerebral TSPGni and FFRni assessment may be a potential diagnostic tool for making clinical decisions of revascularization.

\section{Session 03: Epidemiology}

\section{1}

The Epidemiological Prevalence of Epilepsy in the Nakhichevan Autonomous Republic of Azerbaijan

Aliev G. ${ }^{\text {a }}$ Magalov S. ${ }^{\text {, }}$,Hasanov N. ${ }^{c}$, Hasanov E. ${ }^{c}$

${ }^{a}$ GALLY International Biomedical Research Institute Inc.and School of Health Science and Healthcare Administration, University of Atlanta, GA, USA; ${ }^{b}$ Azerbaijan Medical University, Baku, Azerbaijan; 'Sharur Central District Hospital, Sharur, The Nakhichevan Autonomous Republic

Background: Epilepsy is a devastating neurological disorder in which those afflicted can gain benefit from specific treatments based on their genetics and geographic location. Currently the prevalence of epilepsy is estimated at $0.5-3 \%$ of the world-wide population, and is increasing in developing countries.

Methods: In order to make more accurate assessments of the prevalence of epilepsy, we applied the capture-recapture method in a 12-month study of epilepsy in a population from the Sharur district of Nakhichevan Autonomous Republic, Azerbaijan with 114,451 people from sixty-nine villages and towns. The following methods were applied to determine epilepsy: Door To Door (DTD) survey, Non-Medical Source (NMS), and Medical Source (MS).

Results: Using these three techniques, 1, 032 patients with epilepsy $(9.02 / 1000)$ were included in our study. The DTD survey determined 627 patients with epilepsy (5.48/1000) and 247 $(2.16 / 1000)$ from the NMS methods versus 158 (1.38/1000) from the MS methods. Applying the capture-recapture method (CRM), NMS, and DTD methods, the number of epilepsy cases increased to $1,330(11.62 / 1000)$ [95\% CI: 10.21-13.03]. The combinations of these methods show that 887 people $(7.75 / 1000)$ have a diagnosis of epilepsy.

Conclusion: Our conclusion is that, multiple epidemiological tasks used in our study can be used to estimate clinical signs and/ or markers in future applications for the determination and development of treatment strategies for this devastating disease in the post soviet countries such as the Nakhichevan Autonomous Republic of Azerbaijan. 


\section{6}

Incidence of Traumatic Brain Injury Across the Full Disease Spectrum: A Population-Based Medical Record Review Study

Brown A. a , Leibson C. ${ }^{a}$, Ransom J. ${ }^{a}$, Diehl N. ${ }^{a}$, Perkins P. ${ }^{\mathrm{a}}$, Mandrekar J. ${ }^{\mathrm{a}}$, Malec J. ${ }^{\mathrm{b}}$

aMayo Clinic, USA; ${ }^{\text {b} R e h a b i l i t a t i o n ~ H o s p i t a l ~ o f ~ I n d i a n a ~ a n d ~}$ Indiana University School of Medicine, USA

Background: Extremely few objective estimates of traumatic brain injury incidence include all ages, both sexes, all injury mechanisms, and the full spectrum from very mild to fatal events.

Methods: We used unique Rochester Epidemiology Project medical records-linkage resources, including highly sensitive and specific diagnostic coding, to identify all Olmsted County, MN, residents with diagnoses suggestive of traumatic brain injury regardless of age, setting, insurance, or injury mechanism. Provider-linked medical records for a $16 \%$ random sample were reviewed for confirmation as definite, probable, possible (symptomatic), or no traumatic brain injury. We estimated incidence per 100, 000 person-years for 1987-2000 and compared these recordreview rates with rates obtained using Centers for Disease Control and Prevention (CDC) data-systems approach. For the latter, we identified all Olmsted County residents with any CDC-specified diagnosis codes recorded on hospital/emergency department administrative claims or death certificates during 1987-2000.

Results: Of sampled individuals, 1257 met record-review criteria for incident traumatic brain injury; 56\% were ages 16-64 years, $56 \%$ were male, and 53\% were symptomatic. Mechanism, sex, and diagnostic certainty differed by age. The incidence rate per 100, 000 person-years was 558 (95\% confidence interval _ 528-590) versus 341 (331-350) using the CDC data-system approach. The CDC approach captured only $40 \%$ of record-review cases. Seventy-four percent of missing cases presented to the hospital/emergency department; none had CDC-specified codes assigned on hospital/ emergency department administrative claims or death certificates; and $66 \%$ were symptomatic.

Conclusion: Capture of symptomatic traumatic brain injuries requires a wider range of diagnosis codes, plus sampling strategies to avoid high rates of false-positive events.

7

\section{Cardiovascular Events During World 2006 and European 2008 Football Cups in France}

Aboa-Eboulé C. ${ }^{a}$, Catherine Q. ${ }^{b}$, Lorgis L. ${ }^{c}$, Cottin Y. ${ }^{c}$, Henrotin J.-B. ${ }^{a}$, Giroud M. ${ }^{a}$, Béjot Y. ${ }^{a}$

${ }^{a}$ Registre dijonnais des AVC, EA 4184, IFR 100 Santé-STIC, Dijon, France; 'bervice de Biostatistique et d'Informatique Médicale, Inserm U866, Dijon, France; 'Service de Cardiologie, Hôpital du Bocage, Dijon, France

Background: Stress induced by football competitions is inconsistently associated with an increase in acute cardiovascular events. We examined this hypothesis using French data surrounding World Cup 2006 and European Championship 2008.

Methods: All admissions of patients aged 18 and more for stroke or myocardial infarction (MI) from 2005 to 2009 were retrospectively retrieved from the French Hospital discharge database (FHDDB). Two periods of exposure were analysed: the month of the competitions (exposed) compared (1) to the previous and following months (unexposed) and (2) to the same month of the previous and following years (unexposed) of the football cup. The number of admissions was compared between unexposed and exposed periods using Poisson regression with ratios of admissions (RR) and 95\% CI.

Results: A total of 17811,17680 and 17321 admissions for stroke and 23134, 22413 and 20844 admissions for MI were retrieved during the previous, exposed and, following months. The number of strokes increased by $9 \%$ during the 2006 exposed month but decreased by $5 \%$ during the 2008 exposed month compared to the following months (respective adjusted $\mathrm{RR}=0.91$, $95 \% \mathrm{CI}=0.88-0.94, \mathrm{p}<0.0001 ; \mathrm{RR}=1.05,95 \% \mathrm{CI}=1.02-1.08 ; \mathrm{p}$ $=0.003$ ).

Regardless of the football cup, the number of MI decreased gradually from the previous, the exposed to the following months. Admissions were significantly lower during the exposed month compared to the same month of the following year for stroke or the previous year for MI.

Conclusion(s): The results concerning 2006 World Cup suggest that a defeat by penalty shoot-out in final of the national team increases stroke admissions particularly in susceptible spectators with known cardiovascular risk factors. However, the negative results after the short elimination of the French team in 2008 may be explained by a pre-competition excitement drop. Decline in MI admissions seems unrelated to football competitions but may be explained by better preventive strategies.

\section{4}

\section{High Prevalence of Renal Dysfunction in Acute Stroke Patients in Sub-Saharan Africa: Comparison with a French Stroke Unit}

\author{
Bugnicourt J.-M. ${ }^{a}$, Tchouata Ngandjouc L. ${ }^{\text {, }}$ Chillon J.-M.b, \\ Beugre K. ${ }^{c}$, Lamy C. ${ }^{\text {, }}$ Godefroy O. ${ }^{\text {a }}$ \\ aAmiens Univ. Hospital, Amiens, France; bINSERM U1088, \\ Amiens, France; 'Cocody Univ. Hospital, Ivory Coast
}

Background: In acute stroke patients, the prevalence of chronic kidney disease (CKD) is estimated around 25\%. Although Sub-Saharan African (SSA) subjects undergo epidemiological and demographic transition, the association of renal function and stroke is poorly investigated in this population. In this study, we aimed to compare the prevalence of vascular risk factors (VRF) and renal function, in stroke patients hospitalised in a SSA hospital (Cocody University Hospital, Abidjan, Côte d'Ivoire) and in a French stroke unit (Amiens University Hospital, Amiens, France).

Methods: This retrospective study included all consecutive stroke patients admitted between January and May 2008 (Cocody University Hospital, Abidjan, Côte d'Ivoire) and between October 
and December 2008 (Amiens University Hospital, Amiens, France). We assessed each patient's demographic details, VRF and computed tomography confirmed pathological stroke type. The glomerular filtration rate (eGFR) was estimated using the fourcomponent Modification of Diet in Renal Disease MDRD equation and CKD was defined as an eGFR $<60 \mathrm{ml} / \mathrm{min} / 1.73 \mathrm{~m} 2$.

Results: One hundred and ninety five patients were included (Amiens: 92; Abidjan: 103). French patients were significantly older (68 vs 59 years, $\mathrm{p}<0.001$ ) and had more previously known VRF than African patients. In contrast, African patients had more VRF discovered during hospitalisation. More African than French patients had cerebral haemorrhage $(34 \%$ vs $8 \%, \mathrm{p}<0.001)$ but acute stroke mortality was similar in the two hospitals. Finally, almost $1 / 3$ of acute stroke patients had CKD. CKD was more common in SSA patients than in French patients (43\% vs $24 \%$, p < $0.001)$.

Conclusion(s): Although this was a hospital-based study, CKD appears to be very common in SSA acute stroke patients. These results warrant confirmation in prospective studies.

\section{6}

\section{Antenatal Factors Associated with Perinatal Arterial} Ischemic

Darmency-Stamboul V. , Chantegret $C .{ }^{a}$, Ferdynus $C .{ }^{b}$, Mejean N. ${ }^{c}$, Durand C. ${ }^{c}$, Sagot P. ${ }^{\mathrm{d}}$, Giroud M. ${ }^{\mathrm{e}}$, Béjot Y. ${ }^{f}$, Gouyon J.-B. ${ }^{\mathrm{f}}$

${ }^{a} \mathrm{CHU}$ de Dijon, Service de Pédiatrie; ${ }^{\mathrm{b}} \mathrm{CHU}$ de Dijon, Service de Pédiatrie; ${ }^{\mathrm{C}} \mathrm{CHU}$ de Dijon, Service de Biostatistique; ${ }^{\mathrm{d}} \mathrm{CHU}$ de Dijon, Service de Radiologie; ${ }^{\mathrm{C}} \mathrm{CHU}$ de Dijon, Registre dijonnais des AVC, EA 4184, IFR 100 Santé-STIC; ${ }^{f} \mathrm{CHU}$ de Dijon, Service de Neurologie; ${ }^{9} \mathrm{CHU}$ de Dijon, Service de Néonatologie

Background: Perinatal arterial ischemic stroke (PAIS) is a common cause of hemiplegic cerebral palsy in children. The diagnosis of PAIS is based on cerebral imaging. The objective of our study was to determine prenatal risk factors associated with PAIS.

Methods: A retrospective case-control study nested in the whole population of Burgundy, France, from January 2000 to December 2007. Case patients were confirmed by review of brain imaging and medical records. Three controls per case were randomly selected from the study population, by sex, term, place and year of birth.

Results: PAIS was confirmed in 32 patients and its incidence was $1 / 4400$ live births. In comparison to controls clinical conditions significantly associated to cases were gestational diabetes ( $16.1 \%$ vs $4.2 \%$; $\mathrm{p}=0.04)$, fetal heart rate abnormalities $(35.5 \%$ vs $10.9 \% ; \mathrm{p}=0.001)$ and meconium-stained liquor $(40 \%$ vs $12 \%$; $\mathrm{p}$ $0.001)$. At the limit of statistical significance were found maternal smoking before (39.3 vs $22.9 \%$; $=0.08$ ) and during pregnancy $(32.1 \%$ vs $16.7 \%$; $=0.07)$, cord abnormalities ( $29 \%$ vs $14.1 \%$; $=$ $0.06)$ and caesarean section $(28.1 \%$ vs $14.6 \%$; $=0.08)$. In the multivariate analysis, maternal smoking during pregnancy (OR: $3.1 ; 95 \% \mathrm{CI}, 1.1-8.8 ; \mathrm{p}=0.04$ ) was the only risk factor significantly associated to PAIS.

Conclusion(s): This study is the first to identify maternal smoking during pregnancy as an independent prenatal risk factor of PAIS. Additional prospective studies are needed to confirm this result and to investigate the role of maternal smoking in fetal and neonatal thrombogenesis.

\section{2}

Features of Acute Ischemic Stroke with Rheumatic Heart Disease in a Hospitalized Chinese Population

Wang D., Liu M., Hao Z., Tao W.

West China Hospital, Sichuan University, China

Background: Rheumatic heart disease (RHD) is an important risk factor for ischemic stroke in developing countries. Observational data on its characteristics and influence on outcome or recurrence of ischemic stroke is scarce. We aimed to investigate the proportion, characteristics, functional outcome and recurrence of acute ischemic stroke patients with RHD in a hospitalized Chinese population.

Methods: We prospectively enrolled consecutive patients with acute ischemic stroke who were admitted within one month of stroke onset from January, 2003 to February, 2007 into the analysis. Clinical characteristics such as age, gender, risk factors, NIHSS on admission were investigated. Basic characteristics, functional outcomes and recurrence were compared between RHD group and non-RHD group.

Results: Of the 1638 cases included, RHD was present in 130 patients $(7.9 \%)$. RHD patients, compared with those without $\mathrm{RHD}$, were younger, were more frequently female, and more often had experienced AF and higher NIHSS score on admission (all $\mathrm{P} \leq 0.006$ ). After adjustment for age, sex and NIHSS on admission, the death risk in patients with RHD was 2.0-fold higher at three months, six months and one year compared to patients without RHD (all $\mathrm{P} \leq 0.013$ ). Compared with patients without RHD, those with RHD showed a significantly higher cumulative recurrence rate $(13.6 \%$ versus $6.0 \%, \mathrm{p}=0.001, \log$ rank test) by one year cumulative recurrent curves.

Conclusion(s): Stroke due to RHD is still common in Chinese population. RHD was associated with two fold increased risk of death and one fold increased risk of recurrence in stroke patients. 
24

\section{Epidemiology of Headaches in Adolescence Population of Tehran Urban Area: A Population- Based Study in District 8, Year 2010}

Fereshtehnejad S.-M. ${ }^{\text {a }}$, Shahbeigi S. ${ }^{\text {b }}$, Mohammadi N. ${ }^{c}$, Golmakani M.M. ${ }^{\mathrm{d}}$, Tadayyon S. ${ }^{\mathrm{e}}$, Jalilzadeh G. ${ }^{\mathrm{e}}$, Pakdaman H. ${ }^{\mathrm{b}}$

${ }^{a}$ Karolinska Institutet, Sweden; 'bShaheed Beheshti University of Medical Sciences, Iran; 'Tehran University of Medical Sciences, Iran; ${ }^{\mathrm{d}}$ Health Deputy of Tehran Municipality, Iran;

eJondishapour Neurology Research Center (JNRC), Iran

Background: Many population-based studies have been performed to determine the prevalence of different types of headaches; however, none of them was performed in Tehran urban area as a huge and crowded metropolitan with multiple serious problematic crises. This study was performed to evaluate the prevalence rates of different types of headache among adult population of Tehran urban area in the year 2010.

Methods: In this cross-sectional survey, a "face-to-face, indoor" structured interview was developed and used in district 8 of Tehran urban area in the year 2010. A form concerning the prevalence of different types of headaches which also comprised the characteristics of the headaches and sociodemographic data was designed. Subjects were diagnosed in accordance to the most frequent type of headache experienced in the last 1 year, using ICDH-3 criteria.

Results: Of 3655 recruited individuals, 2778 (76\%) people have experienced headache within last year. Tension and migraine headaches were the most common types with the prevalence of 48.6\% ( $\mathrm{n}=1777)$ and $18.2 \%(\mathrm{n}=665)$, while, drug-induced, chronic daily $(\mathrm{CDH})$ and cluster headaches were presented in $4.9 \%(\mathrm{n}=180), 3.3 \%(\mathrm{n}=122)$ and $0.1 \%(\mathrm{n}=3)$, respectively. Migraine was significantly more occurred in females (22\%) than males $(10.6 \%)[\mathrm{OR}=2.38$ (95\% CI: $1.93-2.93), \mathrm{P}<0.001]$ and the mean age of migrainous subjects was significantly lower than the ones without migraine $[34.66(\mathrm{SD}=12.33)$ yr vs. $36.14(\mathrm{SD}=$ 15.40) yr, $\mathrm{P}=0.009$ ].

Conclusion(s): The prevalence of primary headaches in a sample of Tehran adult population is considerable. This high prevalence of headaches necessitates further evaluation of possible risk factors derived from leaving in such a crowded metropolitan area.
25

Determination of the Prevalence and Associated Factors of Restless Legs Syndrome (RLS) in Iranian Non-Traumatic Patients Who Referred to Neurology and Orthopedic Clinics

Fereshtehnejad S.-M. ${ }^{a}$, Zamani B. ${ }^{b}$, Jamali Danesh Y. ${ }^{\mathrm{b}}$, Bakhti S. ${ }^{\mathrm{b}}$, Mehrabani $\mathrm{M}^{\mathrm{b}}$

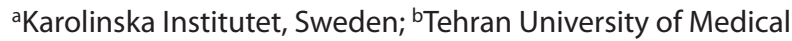
Sciences, Iran

Background: Restless legs syndrome (RLS) is a poorly understood sensory-motor neurological disorder whose prevalence in Caucasian populations ranges from $10-15 \%$. RLS could affect patients' quality of life and may need to be treated. Although common, RLS often escapes diagnosis and many patients are referred to different clinics. Therefore, the aim of this study is to evaluate the prevalence and associated factors of restless legs syndrome (RLS) in Iranian patients who referred to neurology and orthopedic clinics.

Methods: A total of 1103 non-traumatic patients consecutively admitted to the neurology and orthopedic outpatient clinics of Rasoul-e-Akram, Shafa Yahyaeian and Milad hospitals in Tehran, Iran were studied. The diagnosis of RLS was based on the criteria adopted by the International Restless Legs Syndrome Study Group Rating Scale (IRLS). Other variables such as demographics, chief complaint, family history of RLS and systemic diseases were recorded in the check list.

Results: Out of 1103, RLS was detected in 116(10.5\%) patients. The prevalence of RLS among patients who referred to neurology and orthopedic outpatient clinics was $9.7 \%$ and $11 \%$, respectively, whereas, only $3(2.7 \%)$ patients were admitted with chief complaint of RLS symptoms. The prevalence of RLS was significantly higher in women than men $(15 \%$ vs. $4.3 \%, \mathrm{P}<0.001)$ and the mean age of RLS patients was significantly higher than non-RLS patients $[47.9(\mathrm{SD}=15.34)$ years vs. $40.71(\mathrm{SD}=17.8)$ years, $\mathrm{P}<$ 0.001].

Conclusion(s): The results of our study show the considerable prevalence of RLS in Iranian patients. However, most of them are referred to orthopedic clinics than neurology. It seems that increasing both patients and physicians knowledge about RLS may lead to better diagnosis, treatment and improvement of RLS symptoms. 


\section{9}

Acute Stroke in Nógrád County, Hungary, 2005 Female Mortality Is Higher, But Surviving Female Are Less Disabled

\section{Szucs A., Óváry C. ${ }^{a}$, Garancsi G. ${ }^{b}$, Szabó G. ${ }^{c}$}

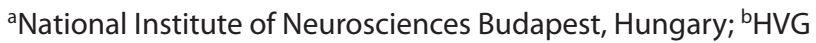
Editorial Office, Hungary; ${ }^{\circ}$ Dr. Kenessey Albert Hospital, Hungary

Background: The pattern of healthcare-use of the two genders as well as features establishing a diagnosis of many diseases differs in the two genders. These non-biologica factors may contribute to different success of diagnosis and unequal outcome of pathologic conditions in the two genders. We wanted to analyse the risk factors, etiologic types and outcome in a cohort of acute stroke inpatients in Nógrád, a poor, relatively underdeveloped county with high health risks in Hungary.

Methods: The data of 646 consecutive patients admitted to the neurology/stroke departments of the county Nógrád for acute stroke during 6 months in 2005 were collected using the HungaroStudy database questionnaire. The data were statistically analyzed by the Statistical Package for the Social Sciences (SPSS) method.

Results: Haemorrhagic stroke made up $10 \%$ of all cases, the overall stroke mortality within 28 days was $30 \%$. Most patients carried several known risk factors for stroke (average 3.6); the number of risk factors correlated with negative outcome expressed by values of the Rankin scale on the 28th day after stroke. Stroke outcome was negatively influenced by advanced age, low educational level, lack of physical activity as well as hopelessness, lack of interest/inactivity and inability to work. The only statistically significant factor however, for stroke outcome in this cohort was the gender: mortality was higher in women; but surviving women had significantly better outcome than men.

Conclusion(s): Acute stroke in our multi-risk population had high mortality and relatively poor outcome; the number of risk factors correlated with bad outcome. Similarly to myocardial infarction, women had higher stroke mortality than men, however, if surviving, female gender seems to be a protective factor for better outcome.

\section{6}

Epidemiology and Management of Stroke in Western Ukraine: Results from a Population-Based Stroke Registry

Filipets 0 .

Bukovinian State Medical University, Ukraine

Background: According to official statistical reports, Ukraine has some of the highest stroke morbidity rates in Europe. However, there is a lack of studies that provide reliable information on key indicators of stroke burden such as incidence and case fatality.

Methods: A population-based stroke registry in the city of Chernivtsi, Western Ukraine (240 476 inhabitants) was set up to evaluate epidemiology of stroke during 2006-2007. All first-ever stroke events were registered and assessed prospectively using standard WHO definitions.

Results: Over the study period, a total of 1080 cases of firstever stroke were identified. Mean (SD) age of patients was 66.7 (12.6) years and 550 (50.9\%) were women. The average crude incidence rate was $224.6 / 100000$ (95\% CI 206.2-244.5), in men $240.1 / 100000$ (212.4-271.2), in women 211.5/100000 (187.5238.3), $\mathrm{p}=0.139$. After adjustment to the world population, the values were 176.1, 223.5 and $143.8 / 100000$ respectively. $84.0 \%$ of patients were admitted to hospitals. Of these, $32.4 \%$ arrived within 3 hours after symptom onset. Only $30.6 \%$ of persons diagnosed with stroke had undergone cerebral imaging that hampered the definition of total proportion of stroke subtypes. None of the patients received thrombolysis. Within 28 days of the onset 296 deaths from stroke were recorded. The average crude mortality rate was 61.5/100000 (95\% CI 52.2-72.5). Overall 28-day case fatality was $27.4 \%$.

Conclusion(s): The present study adds to the evidence that effective stroke surveillance system needs to be established in Ukraine. It will allow for planning prevention strategies, decreasing stroke incidence as well as improving stroke management, e.g., reducing delay in healthcare delivery and increasing availability of neuroimaging procedures.

\section{7 \\ Geomagnetic Disturbances May Be Environmental Risk Factor for Multiple Sclerosis: An Ecological Study for Preliminary Evaluation of a New Hypothesis}

Sajedi Seyed A., Abdollahi F.

Ahvaz Jundishapur University of Medical Sciences, Iran

Background: We noticed that a hypothesis based on the effect of geomagnetic disturbances (GMD) has the ability to provide probable explanations for special features of multiple sclerosis (MS) such as the cause of its relapsing-remitting nature, MS incidence and prevalence trend, the birth time effect, and MS epidemics. Areas around geomagnetic 60 degree latitude (GM60L) experience the greatest amount of GMD. The easiest way to evaluate our hypothesis was to test the association of MS prevalence (MSP) with "angular distance to geomagnetic 60 degree latitude" (AMAG60) and compare it with the known association of MS with geographical latitude (GL). We did the same with "angular distance to geographic 60 degree latitude" (AGRAPH60) as a control.

Methods: English written papers with MSP keywords, done in Europe (EUR), North America (NA) or Oceania (OC) were retrieved from the PubMed. Geomagnetic coordinates were determined for each location and AMAG60 was calculated as absolute value of numerical difference between its geomagnetic latitude from GM60L. Only studies were entered in the analyses that used an approved MS diagnostic criterion with more than 20 prevalent cases and clearly indicated the number of prevalent cases. For studies that used patients' self-report of having MS according to 
their neurologists' diagnosis, we accepted the data only if the study population were such large that choosing self-report method seemed practically inevitable and reasonable. By an ecological study with using meta-regression analyses, relationship of MSP with GL, AMAG60 and AGRAPH60 were evaluated separately. MSP data were weighted by square root of number of prevalent cases. Models were compared by their adjusted R square $(A R \wedge 2)$ and standard error of estimate (SEE).

Results: $111 \mathrm{MSP}$ data were entered in the study. In each continent, AMAG60 had the best correlation with MSP, the largest $\mathrm{AR}^{\wedge} 2$ (0.47, 0.42 and 0.84 for EUR, NA and OC, respectively) and the least SEE. Merging both hemispheres data, AMAG60 explained $56 \%$ of MSP variations with the least $\mathrm{SEE}(\mathrm{R}=0.75, \mathrm{AR} \wedge 2=0.56$, $\mathrm{SEE}=57)$, while GL explained $17 \%\left(\mathrm{R}=0.41, \mathrm{AR}^{\wedge} 2=0.17, \mathrm{SEE}=\right.$ 78.5) and AGRAPH60 explained $12 \%$ of those variations with the highest SEE $\left(\mathrm{R}=0.35, \mathrm{AR}^{\wedge} 2=0.12, \mathrm{SEE}=80.5\right)$.

Conclusion(s): Our results confirmed that AMAG60 is the best describer of MSP variations and has the strongest association with MSP distribution. They clarified that the well-known latitudinal gradient of MSP may be actually a gradient related to GM60L. Moreover, the location of GM60L can elucidate why MSP has parabolic and linear gradient in the north and south hemisphere, respectively. This preliminary evaluation supported that GMD may be an important environmental risk factor for MS. We believe that this hypothesis deserves to be considered for further validation studies.

\section{8 \\ Mortality and Factors Associated with Mortality in People Living with Convulsive Epilepsy in a Rural Area of Kenya}

Ngugi A. ${ }^{a}$, Bottomley $C .{ }^{b}$, Fegan $G .{ }^{a}$, Kleinschmidt I. ${ }^{b}$, Newton $C .{ }^{a}$

${ }^{a}$ Centre for Geographical Medicine Research -Coast, KEMRI, Kenya; ${ }^{b}$ London School of Hygiene and Tropical Medicine, United Kingdom

Background: Most epilepsy mortality studies from Low and Middle Income Countries (LMIC) are based on small cohorts while some have been conducted in areas with high incidence of epilepsy. Also, there are no studies on risk factors of mortality in people with epilepsy. We measured mortality and identified associated factors in a cohort of people with active convulsive epilepsy (ACE) in a rural area of Kenya.

Methods: People with ACE were identified in a cross-sectional survey and followed up quarterly for three years to collect information on putative risk factors of mortality. We estimated case fatality proportion (CFP), mortality ratios and standardized mortality ratio (SMR). We used Poisson regression analysis to examine the influence of potential risk factors on mortality.

Results: We registered 61 deaths among 754 people with ACE $(\mathrm{CFP}=8.1 \%)$. Mortality rate was 33.3/100, 000/year $(95 \% \mathrm{CI}$ : 25.9-42.8). The SMR was 6.5 (95\% CI: 5.0-8.3) and ACE mortality was higher in for all age groups. Non-adherence to treatment was associated with mortality in this population; adjusted Odds Ratio was 4.7 (95\% CI: 2.2-10.4).
Conclusion(s): Epilepsy confers an additional risk of mortality in this population and is associated with non-adherence to treatment which is amenable to public health intervention.

\section{4 \\ The Validation of a Three-Stage Screening Methodology for Detecting Active Convulsive Epilepsy in Population-Based Studies in Health and Demographic Surveillance Systems}

\author{
Ngugi A. ${ }^{a}$, Bottomley C. ${ }^{\text {, }}$, Chengo E. ${ }^{a}$, Kombe M. ${ }^{a}$, Kazungu M. ${ }^{\text {a }}$ \\ Bauni E. ${ }^{\mathrm{a}}$ Mbuba C. ${ }^{\mathrm{a}}$, Kleinschmidt I. ${ }^{\mathrm{b}}$, Newton $\mathrm{C}^{\text {a }}$ \\ ${ }^{a}$ Centre for Geographical Medicine Research-Coast, KEMRI, \\ Kenya; 'bondon School of Hygiene and Tropical Medicine, \\ United Kingdom
}

Background: There are few studies on the epidemiology of epilepsy in large populations in Low and Middle Income Countries (LMIC). Most studies in these regions use two-stage populationbased screening surveys, which are time-consuming and costly to implement in large populations required to generate accurate estimates. We examined the sensitivity and specificity of a three-stage cross-sectional screening methodology in detecting active convulsive epilepsy (ACE), which can be embedded within on-going census of demographic surveillance systems.

Methods: We validated a three-stage cross-sectional screening methodology on a randomly selected sample of participants of a three-stage prevalence survey of epilepsy. Diagnosis of ACE by an experienced clinician was used as 'gold standard'. We further compared the expenditure of this method with the standard two-stage methodology.

Results: We screened 4442 subjects in the validation and identified 35 cases of ACE. Of these, 18 were identified as false negatives, most of whom (15/18) were missed in the first stage and a few $(3 / 18)$ in the second stage of the three-stage screening. Overall, this methodology had a sensitivity of $48.6 \%$ and a specificity of $100 \%$. It was also $60 \%$ cheaper than a two-stage survey.

Conclusion(s): This was the first study to evaluate the performance of a multi-stage screening methodology used to detect epilepsy in demographic surveillance sites. This method had poor sensitivity attributed mainly to non-response in the first stage. This method needs to take into consideration the poor sensitivity and the savings in expenditure and time as well as validation in target populations. 


\section{5}

\section{Epidemiology of Primary Brain Tumours in Georgia: Two-Year Results of a Prospective Population-Based Study}

Gigineishvili D., Shegelia N., Kartsivadze S., Shalashvili G., Tsiskaridze A., Shakarishvili R.

Tbilisi State University, Faculty of Medicine, Georgia

Background: The aim of this study was to determine the incidence patterns of primary brain tumours.

Methods: A population-based cohort study of all patients diagnosed with primary brain tumours was set-up in Georgia starting in April 2009. Data from medical records of 11 hospitals and brain scan reports from 9 imaging units were collected and reviewed. Follow-up scan reports and tumor recurrent cases were excluded.

Results: 981 new cases were detected during the first two years of the study; males accounted for $44.2 \% .83$ cases were diagnosed in children ( $<20$ years). Annual incidence rate adjusted to WHO world standard population was 9.72 per 100, 000 person-years. $60 \%$ of all tumors were diagnosed neuroradiologically. Nonmalignant tumors accounted for $65 \%$ of all tumors (3.23 per 100 , $000)$. The most common histology was meningioma (43.6\%), followed by pituitary tumors (22.3\%). Among major histology groups, crude incidence rates were highest for tumors of the meninges $(3.22$ per 100,000$)$, followed by tumors of the sellar region $(1.47$ per 100,000$)$ and neuroepithelial tumours $(1.47$ per $100,000)$. Incidence rates by specific histology were highest for meningioma (2.88 per 100,000$)$, pituitary adenomas (1.30 per $100,000)$ and glioblastomas $(0.54$ per 100,000$)$. Crude rates of all major histology groups are higher among females than males.

Conclusion(s): This is the first epidemiological study of primary brain tumours in the Caucasus region based on current 2007 WHO classification. The observed low incidence rates by tumour behavior compared with international published data may be explained by high percentage of unclassified tumours (40.6\%) due to extensive use of neuroimaging as a diagnostic modality.

Supported by the S. Rustaveli National Science Foundation (grant\#08_207_6_329).

\section{7}

Spatial Distribution of Cerebral White Matter Lesions Predicts Progression to $\mathrm{MCl} /$ Dementia

Mortamais M. ${ }^{a}$, Reynes C. ${ }^{b}$, Brickman A. ', Provenzano F.c, Muraskin J. ', Portet F. , Berr C. ${ }^{\text {, }}$, Touchon J. ${ }^{a}$, Bonafé A. ${ }^{d}$, Le Bars E. ${ }^{\text {, }}$ Maller J. ${ }^{\mathrm{e}}$, Meslin C. ${ }^{\mathrm{f}}$, Sabatier R. ${ }^{\mathrm{b}}$, Ritchie K. ${ }^{\mathrm{a}}$, Artero S. ${ }^{\mathrm{a}}$

aINSERM U1061, Montpellier, France; 'bniversité Montpellier1, France; 'Taub Institute for Research on Alzheimer's Disease and the Aging Brain, USA; ${ }^{d} \mathrm{CHU}$ Montpellier, France; ${ }^{\mathrm{e}}$ Monash Alfred Psychiatry Research Centre, The Alfred \& Monash University School of Psychology and Psychiatry, Australia; ${ }^{f}$ Centre for Mental Health Research, Australian National University, Australia

Background: White matter lesions (WML) increase the risk of dementia. The relevance of location of WML with respect of dementia is less clear. The objectives of this study were to determine whether a particular profile of WML distribution, characterized by density and location, might be associated with the increased risk of developing either mild cognitive impairment (MCI) or dementia over the following 7 years.

Methods: The study population consisted of 426 healthy subjects from a cohort of community-dwelling persons aged 65 years and over (Esprit project). Standardized cognitive and neurological evaluations were repeated at 2, 4 and 7 year follow-up. We examined the patterns of WML using a supervised data mining approach (decision trees) with regional WML volumes (frontal, parietal, temporal, and occipital regions) and total WML volume estimated at baseline. Cox proportional hazards models were then constructed to study the association between the patterns of WML and risk of $\mathrm{MCI} /$ dementia.

Results: Total WML volume and percentage of WML in the temporal region were the best predictors of subsequent MCI and dementia. The pattern characterized by a severe WML load with a high proportion of lesions in the temporal region was significantly associated with the risk of developing MCI or dementia, independently of potential confounders.

Conclusion(s): Above a certain threshold of damage, WML cluster in the temporal region in some subjects, who are more likely to become MCI or demented. This distribution is observed before the onset of clinical symptoms, which could facilitate the detection of patients at risk of $\mathrm{MCI} /$ dementia.

\section{8 \\ Vestibular Schwannoma: A 1:1 Matched Case-Control Study of the Environmental Risk Factors and Epidemiology}

Berkowitz O., lyer A., Talbott E., Lunsford L.D.

University of Pittsburgh, USA

Background: There is little known about the etiology of vestibular schwannomas. The goal of this study has been to describe the epidemiology of vestibular schwannoma and explore some of 
the potential risk factors that are not yet well understood such as medical history, tobacco and social history, environmental exposures, and occupational history.

Methods: A hospital based case-control study with 1:1 matching based on age and gender was designed with a written questionnaire format. Multiple conditional logistic regression models were created to determine odds ratio (OR).

Results: A total of 353 matched pairs showed the average age at diagnosis was 53 (StDev \pm 12 ). More than $90 \%$ of the participants were Caucasian. Patients were evenly distributed by gender and $73.1 \%$ of cases reported ( $\geq 13$ years) of education compared with $49.6 \%$ of controls. Multivariate models revealed significantly increased associated risk in the following variables: Family history of cancer $(\mathrm{OR}=1.8295 \% \mathrm{CI}=1.04-3.18)$, history of hay fever (OR $=3.91,95 \% \mathrm{CI}=1.35-11.30)$, and managerial/professional occupations $(\mathrm{OR}=4.41,95 \% \mathrm{CI}=2.07-9.40)$. Tobacco use was found to have a protective effect $(<20$ pack years $\mathrm{OR}=0.10,95 \% \mathrm{CI}=$ $0.04-0.28 ; \geq 20$ pack years $\mathrm{OR}=0.0395 \% \mathrm{CI}=0.01-0.12)$. Loud noise and chemical exposure were not significant.

Conclusion(s): Patients with vestibular schwannoma have the profile of being Caucasian, either gender, in their 50-60's, and working in managerial, professional jobs. Better healthcare access might have a role in the diagnosis of this tumor. Hay fever and family history of cancer are strongly associated with an increase risk of vestibular schwannoma. Tobacco use demonstrates a protective effect but this mechanism is poorly understood and tobacco remains a significant public health problem.

\section{9 \\ The Associated Risk of Cell Phone Use and Vestibular Schwannoma: A Case Control Study}

Berkowitz O., Han Y.-Y., Talbott E., Lunsford L.D.

University of Pittsburgh, USA

Background: The use of cellular phone technology has increased dramatically across the globe. Much interest has been dedicated to its association with the development of brain tumors. The goal of this study has been to examine the association of cell phones with the development of vestibular schwannoma.

Methods: We performed a case-control study with 353 1:1 matched pairs based on age and gender. Participants were recruited from our neurosurgery database along with hospitalbased controls. A questionnaire format was used. Multivariate conditional logistic regression models were created to estimate odds ratio (OR).

Results: A greater proportion of vestibular schwannoma patients reported ever using cell phone $(\mathrm{OR}=1.67,95 \% \mathrm{CI}=1.17-$ 2.38) but this was not found to be significant in our multivariate analysis model after adjusting for several social factors $(\mathrm{OR}=1.03$, $95 \% \mathrm{CI}=0.63-1.69)$. There was no significant association with analogue vs. digital technology $(\mathrm{OR}=1.77,95 \% \mathrm{CI}=0.96-3.24$; $\mathrm{OR}=0.87,95 \% \mathrm{CI}=0.51-1.46$, respectively). Cell phone usage was not significant for periods over ten years and even over fifteen years in our multivariate model $(\mathrm{OR}=1.38,95 \% \mathrm{CI}=0.75-2.56$; $\mathrm{OR}=1.87,95 \% \mathrm{CI}=0.77-4.55$, respectively). Cordless phone use was not significant $(\mathrm{OR}=0.94,95 \% \mathrm{CI}=0.62-1.41)$.
Conclusion(s): In a large group of patients, we found that cell phone usage was not significantly associated with vestibular schwannoma. No significant relationship could be found with analogue technology or after usage periods of $\geq 15$ years. Although a short term effect could not be found with vestibular schwannoma, the long-term health effects of cell phone use on a variety of biological processes require further long term studies to better understand this question.

\section{2 \\ Epidemiology of Parkinson's Disease and Atypical Parkinsonism in Eastern Europe}

\author{
Winter Y.a , Bezdolnij J. ${ }^{\mathrm{b}}$, Katunina E. ${ }^{\mathrm{b}}$, Oertel W. ${ }^{\mathrm{a}}$, Dodel R. ${ }^{\mathrm{a}}$, \\ Gusev E. ${ }^{\text {b }}$
}

aPhilipps-University Marburg, Germany; ${ }^{\text {b}}$ Russian Medical State University Abstract, Russian Federation

Background: Epidemiological data on Parkinson's disease (PD) and atypical parkinsonian syndromes (APS) in East European countries are limited. The objective of this prospective study was to investigate and compare the epidemiological data on PD and APS in the Russian population.

Methods: Data were collected between 07/2006 and 12/2008 in a large district of Moscow with a population of 1, 237, 900 inhabitants. All cases of PD or APS were identified by a specialist in movement disorders and followed up prospectively to confirm the diagnosis. Disease severity, cognitive function and depression were assessed by the Hoehn and Yahr scale, the Mini-Mental State Examination and the Beck Depression Inventory, respectively.

Results: The age-standardized incidence rates per 100, 000/ year were 9.03 (95\% CI: 8.01-10.15) for PD and 0.21 (95\% CI: 0.09-0.31) for APS. The prevalence of PD was 76.5 per 100, 000. The age-standardized male-to-female ratio of PD was 0.87 for all ages and 1.46 for those aged 60 and older. Depression was present in $51 \%$ and cognitive impairment in $44 \%$ of prevalent PD-cases.

Conclusions: Given the rapid growth of the elderly population in Eastern European countries and Asia, the epidemiology of PD and APS in these regions should be more thoroughly investigated. The incidence and prevalence of PD in our study was slightly lower than in studies of Western populations and the male-tofemale ratio was closer to those reported in studies from Asia. The clinical implication of our study is that it highlights the need for better diagnosis and treatment of non-motor symptoms in PD. 


\section{5 \\ Prevalence of Multiple Sclerosis in Verona, Italy: An Epidemiologic and Genetic Study}

Gajofatto A., Stefani A., Turatti M., Bianchi Maria R., Gomez Lira M., Salviati A., Benedetti M.D.

University of Verona, Italy

Background: Recent multiple sclerosis (MS) prevalence studies have classified Italy as a high-risk area. The aim of this study was to determine MS prevalence in Verona, Italy, and the frequency of myelin oligodendrocyte glycoprotein (MOG) gene G511C polymorphism and HLA-DRB1 ${ }^{\star} 15$ locus in a sample of cases and healthy controls.

Methods: The study area population on the prevalence date (December 31st 2001) was 253, 208 (133, 508 women, 119, 700 men). Multiple case sources were examined and medical records were reviewed to verify the diagnosis. Patients who satisfied the McDonald's criteria (2001) for MS were included. Crude, age- and sex-specific prevalence rates were computed. MOG G511C polymorphism and HLA-DRB $1^{\star} 15$ were determined by standard methods.

Results: We identified 270 MS cases yielding a crude prevalence rate of 106.6/100, 000, 95\%CI: 94-120). Prevalence was higher in women $(140.8 / 100,000)$ than in men $(68.5 / 100,000)$. The age-adjusted prevalence rate standardized to the European population was 96.0/100, 000. Age-specific prevalence rates showed a peak in the age group 35-44 years in women and 55-64 years in men. Median Expanded Disability Status Scale (EDSS) score was 3.0 in women and 3.5 in men (range 0-9.5). MOG G511C polymorphism did not differ between cases and controls. HLA-DRB ${ }^{\star} 15$ frequency was $38 \%$ in cases and $15.4 \%$ in controls $(\mathrm{p}<0.001)$.

Conclusions: The high MS prevalence in Verona is in accordance with the most recent epidemiological findings confirming Italy as a high-risk area for the disease, without evidence of an intra-regional latitudinal effect. While HLA-DRB1 ${ }^{\star} 15$ allele is a relevant MS susceptibility locus in mainland Italian population, susceptibility to autoimmunity is influenced by a genetic modulator other than HLA-DRB1* 15 locus.

\section{7 \\ Prevalence of Recurrent Traumatic Brain Injury (TBI) in a New Zealand Population-Based Incidence Sample}

Theadom A. ${ }^{a}$, Feigin V. ${ }^{a}$, Barker-Collo S.B. ${ }^{b}$, Starkey N. ${ }^{c}$, Jones K. ${ }^{a}$

${ }^{a}$ AUT University, Auckland, New Zealand; bUniversity of Auckland, New Zealand; 'The University of Waikato, Hamilton, New Zealand

Background: Little is known about the prevalence, risk factors and long-term consequences of sustaining more than one (recurrent) TBI in the general population. However, evidence suggests the effects of recurrent TBI are far in excess of what would be expected by the nature of the subsequent injury alone. Knowledge of the risk factors, impact and prevalence of recurrent TBI are essential to inform clinical treatment and preventive efforts.

Methods: All new cases of TBI were identified using prospective and retrospective surveillance methods, in the total population of Hamilton and Waikato districts over a one-year period (2010-2011) as part of the Brain Injury Outcomes New Zealand In the Community (BIONIC) study. To facilitate complete case ascertainment, all new hospitalised and non-hospitalised TBI cases (fatal and non-fatal) were identified through multiple overlapping sources. Consenting participants were followed-up for one-year following injury to assess the occurrence of prior and recurrent brain injuries, neuropsychological functioning, post concussive symptoms and mortality. The impact of recurrent injuries within the follow-up period will be examined as pre and post injury data is available.

Results: Preliminary findings revealed that $35 \%$ of the total sample had sustained $>1$ TBI in their lifetime. There was a higher than anticipated rate of injuries prior to entry in the study $(27 \%)$ and recurrent TBI within the follow up period (8\%). $60 \%$ of recurrent TBIs occurred in males, with highest peaks in prevalence in the under 5's and 18-25year olds. 53\% of recurrent TBIs were classified as mild with a high risk of complications (Servadei et al, 2001). The most frequent mechanisms of recurrent injuries in the year following the registering injury were falls (47\%) and assaults (16\%).

Conclusion: This is the only known epidemiological investigation of the prevalence and impact of recurrent TBI in a population based sample. The preliminary results suggest that greater efforts are needed to prevent recurrent TBIs that predominantly affect children and young adults.

Traumatic brain injury, recurrent brain injury, epidemiology.

\section{8 \\ Circulating IL-6 and the Severity of Dilated Virchow- Robin Spaces in the Elderly

\author{
Doijiri R. ${ }^{a}$, Miyashita K. ${ }^{a}$, Kokubo Y. ${ }^{a}$, Miyamoto Y.a , Uno $H_{.}{ }^{a}$, \\ Naritomi H. ${ }^{\mathrm{b}}$, Nagatsuka K. $^{\mathrm{a}}$, Miyashita K. ${ }^{\mathrm{a}}$ \\ ${ }^{a}$ National Cerebral and Cardiovascular Center, Suita, Japan; \\ benri Chuo Hospital, Japan
}

Background: Acute vertigo or dizziness(V/D) is a common presenting symptom in ambulatory care settings, and stroke is its leading. While approximately $10 \%$ of patients with acute isolated V/D show cerebrovascular lesion on CT or MRI, little has yet been known as to whether isolated V/D patients without cerebrovascular lesion have high risks for stroke. This study aimed to determine the risk of stroke in isolated V/D patients without cerebrovascular lesion. We conducted a case control study to examine the association between those patients and general population.

Methods: The study consisted of 170 patients hospitalized with a principal diagnosis of isolated V/D and no vascular lesion on brain CT and MRI who were admitted during the period between April 1988 and March 2010. Suita city residents without 
V/D who underwent medical examinations for epidemiological Suita study during the period between September 1989 and February 2000 comprised the control group $(n=3970)$. The risk of stroke occurrence in the follow-up period of acute isolated V/D patients was examined by logistic regression analysis adjusting for possible confounding variables.

Results: During 12-year follow-up period, 11 patients with acute isolated V/D (6.4\%) and 70 control subjects (1.8\%) developed ischemic stroke. In multivariate analysis adjusted for age and sex, isolated V/D had the significant relationship with stroke events (odds ratio: 2.80; 95\% confidence intervals (CI): 1.44-5.44; $\mathrm{p}=0.002$ ). The significant relationship between isolated $\mathrm{V} / \mathrm{D}$ and stroke was observed even after adjustment for age, sex, body mass index, hypertension, diabetes mellitus, dyslipidemia, current smoking and excess(> 2 alcoholic units/ day) (odds ratio: 2.21; 95\% CI: $1.07-4.57 ; \mathrm{p}=0.03$ ).

Conclusion(s): The patients with acute isolated V/D and no cerebrovascular lesion showed more than twice higher risk for ischemic stroke than general population. Further studies are warranted for developing better diagnostic and follow-up strategies in those patients after discharge.

\section{3}

\section{Cognitive Investigation Methods in A Multicentric Brazilian Cohort Study of Adults}

Passos V., Giatti L., Caramelli P., Bensenor I., Barreto S.

Faculty of Medicine, Universidade Federal de Minas Gerais, Brazil

Background: We describe the methodology of the Longitudinal Study of Adult Health (ELSA-BRASIL), a multicentric cohort to investigate the incidence and natural history of cardiovascular diseases, diabetes and cognitive impairment among 15,105 adults.

Methods: Every 3 years, the cognitive status of the participants is evaluated using tests of the Brazilian version of the CERAD cognitive battery. Memory isevaluated by word learning and retention tests. $\mathrm{V}$ validated versions of the semantic and phonemic verbal fluency evaluate language, memory and concentration. Executive functions are evaluated by Trail Making Test B. The performance is evaluated taking into account intra-individual changes and the influences of clinical and context variables over time. Baseline examination included interview, anthropometric and BP measures, laboratory exams, ECG, echocardiogram, carotid scan and retinal photos. A repository for biological samples was established. Annual telephone surveillance starting in 2009 will continue for the duration of the study and the first follow up examination is scheduled for 2012-2014.

Results: All participants did the cognitive tests. The cohort compromises $54 \%$ women and $46 \%$ men; $61 \%$ aged between $35-54$ years, $28 \%$ aged $55-64$ years and $11 \%$ aged $65-74$ years; $52 \%$ considered themselves to be white, the remaining to be brown, black, Asian or indigenous. Most participants (7950; 53\%) had at least college education, although 1922(13\%) had not completed secondary education.
Conclusion: The predictive value of biological, behavioral, occupational and psychosocial variables on cognitive decline will be investigated in a multi-ethnic population of a medium-income country.

\section{8 \\ Serum 25-Hydroxyvitamin D Predicts Severity and Prognosis in Stroke Patients}

Béjot Y., Daubail B., Jacquin A., Guilland J.C., Hervieu M., Giroud M.

Registre dijonnais des AVC, EA 4184, IFR 100 Santé-STIC, France

Background: We aimed to evaluate the association between 25-hydroxyvitamin D (25(OH)D) levels and both clinical severity at admission and outcome at discharge in stroke patients.

Methods: From February 2010 to December 2010, consecutive stroke patients admitted to the department of neurology of Dijon, France were identified. Clinical information was collected. Serum concentration of $25(\mathrm{OH}) \mathrm{D}$ was measured at baseline. Stroke severity was assessed at admission using the NIHSS score. Functional impairment was evaluated at discharge using the modified Rankin scale (m-Rankin). Multivariate analyses were performed using logistic regression models.

Results: Of the 386 recorded patients, serum 25(OH)D levels were obtained in 382 (median value: $35.1 \mathrm{nmol} / \mathrm{L}$; IQR = 21-57.8). At admission 208 patients had a NIHSS $\leq 5$, with a higher mean $25(\mathrm{OH}) \mathrm{D}$ level than that observed in patients with moderate-tohigh severity ( 45.9 versus $38.6 \mathrm{nmol} / \mathrm{L}, \mathrm{p}<0.001)$. In multivariate analyses, a $25(\mathrm{OH}) \mathrm{D}$ level in the lowest tertile $(<25.7 \mathrm{nmol} / \mathrm{L})$ was a predictor of a NIHSS $\geq 6(\mathrm{OR}=1.67 ; 95 \%$ CI: $1.05-2.68, \mathrm{p}=$ $0.03)$. The mean $25(\mathrm{OH}) \mathrm{D}$ level was lower in patients with moderate-to-severe handicap at discharge (m-Rankin 3-6) than in patients with no or mild handicap (35.0 versus $47.5 \mathrm{nmol} / \mathrm{L}, \mathrm{p}<$ $0.001)$. In multivariate analyses, the lowest tertile of $25(\mathrm{OH}) \mathrm{D}$ level $(<25.7 \mathrm{nmol} / \mathrm{L})$ was associated with a higher risk of moderate-to-severe handicap $(\mathrm{OR}=2.06$; 95\% CI: 1.06-3.94, $\mathrm{p}=0.03)$.

Conclusion(s): A low serum 25(OH)D level is a predictor of both severity at admission and poor early functional outcome in stroke patients. The underlying mechanisms of these associations remain to be investigated. 


\section{1}

\section{Short-Term and Long-Term Stroke Survival: The Belgrade Prognostic Study}

Gallo V.a , Wark P.A. ${ }^{\mathrm{b}}$, Mazda J. ${ }^{\mathrm{c}}$, Pearce N. ${ }^{\mathrm{d}}$, Brayne C. ${ }^{\mathrm{e}}$, Vermeulen $R^{f}{ }^{\mathrm{f}}$, Andersen P.A. ${ }^{\mathrm{g}}$, Kyrozis A. ${ }^{\mathrm{h}}$, Vanacore $N .{ }^{\mathrm{i}}$

aLondon School of Hygiene and Tropical Medicine, United Kingdom; ' Imperial College London, United Kingdom; International Agency for Research on Cancer, France; 'London School of Hygiene and Tropical Medicine; eUniversity of Cambridge, United Kingdom; fUniversity Medical Centre Utrecht, Netherlands; 9Umeå University, Sweden; ' University of Athens, Greece; 'Italian National Institute of Health, Italy

Background: Clinical observations have consistently reported that patients with Amyotrophic Lateral Sclerosis (ALS) are generally lean with a decrease of energy stores. The aim of this research is to investigate for the first time the association between body fatness and risk of ALS with an appropriate prospective study design.

Methods: The EPIC study included 518, 108 individuals recruited from the general population across 10 Western European countries. At recruitment, information on lifestyle was collected and anthropometric characteristics (height, weight, waist and hip circumference) were measured. Cox hazard models were fitted to investigate the associations between anthropometric measures and ALS mortality.

Results: 222 ALS deaths (79 men and 143 women) occurred during the follow up period (mean 13 years). The interaction between categories of BMI with ALS by gender was statistically significant $(\mathrm{p}=0.009)$ : BMI categories showed a tendency of decreased risk among overweight and obese compared to normal weight men with a significant risk reduction increasing BMI units $\left(\mathrm{HR}=0.93,95 \%\right.$ C.I. $0.86-0.99$ per $\left.\mathrm{kg} / \mathrm{m}^{2}\right)$; similarly the risk significantly increased more than 3-fold for underweight compared to normal weight women. Among women only a significant risk reduction increasing the waist-hip ratio (WHR) was evident: women in the top quartile had a more than halved risk of ALS compared to those in the bottom quartile $(\mathrm{HR}=0.48,95 \%$ C.I. $0.25-0.93)$ with a borderline significant $\mathrm{p}$-value for trend across quartiles $(\mathrm{p}=0.056)$.

Conclusion(s): The present study suggests an inverse association between body fat and risk of ALS mortality, although this showed different patterns in men and women.

\section{4 \\ Dementia in Central Africa}

Preux P.M. ${ }^{\mathrm{a}}$, Guerchet M. ${ }^{\mathrm{a}}$, Mbelesso P. ${ }^{\mathrm{b}}$, Mouanga A. ${ }^{\mathrm{c}}$, Ndamba Bandzouzi B. ${ }^{c}$

aUniversity of Limoges, France; b Bangui University, Central African Republic; 'Brazzaville University Hospital, Congo Rep.

Background: Dementia and related syndromes are of major public health concern because of the ageing of global population. Few researches have been carried out in Africa compared to developed countries parameters.
Methods: A research program on dementia in Central Africa (Central African Republic and Republic of Congo) was performed during the last years. Door to door surveys were carried out in districts of Bangui and Brazzaville. Participants aged $\geq 65$ years old were interviewed for sociodemographical data, medical history and depressive disorders. Subjects who had a poor performance at cognitive tests were clinically assessed by a neurologist. Further psychometrical tests were conducted. The DSM-IV and NINCDSADRDA criteria were required for dementia and Alzheimer's disease diagnoses.

Two years later, all subjects with dementia or cognitive impairment at the initial study and controls free-of-dementia were retrieved. Verbal autopsies and cognitive assessment were carried out.

Results: Prevalence of dementia was $6.7 \%$ in Brazzaville and $8.1 \%$ in Bangui. Increasing age, female gender, hypertension, a $\mathrm{BMI}<18.5 \mathrm{~kg} / \mathrm{m}^{2}$, depressive symptoms and the lack of a primary education were significantly associated with dementia. Death of one parent during childhood and recent moving were also associated with dementia. At the follow-up, crude mortality rates were $27.5 \%$ and $40.0 \%$ in demented subjects respectively in Bangui and Brazzaville, $15.3 \%$ and $12.1 \%$ among elderly with cognitive impairment and $10.7 \%$ and $7.1 \%$ in controls.

Conclusion(s): These results add to the few available figures from developing countries. Comparison of these results with other studies in developed countries could lead to new physiopathological hypotheses.

\section{7}

\section{Prior Transient Ischemic Attack (TIA) and Early Dementia After Subsequent Ischemic Stroke}

Jacquin A. a, Rouaud O. ', Aboa-Eboulé C. , Osseby G.V. , Binquet C. ${ }^{b}$, Giroud M. ${ }^{a}$, Béjot $Y{ }^{a}{ }^{a}$

aRegistre dijonnais des AVC, EA 4184, IFR 100 Santé-STIC, France; ${ }^{b} \mathrm{CIC}-\mathrm{EC}$, France

Background: Whereas functional recovery and survival after ischemic stroke seem to improve in patients with prior Transient Ischemic Attack (TIA), little is known about the effect of prior TIA on post-stroke cognition. Our aim was to evaluate, in a population-based study, the impact of prior TIA on early dementia in patients who survived the first month after first-ever ischemic stroke.

Methods: A total of 1697 non-aphasic patients who survived the first month after first-ever ischemic stroke were identified from the population-based registry of Dijon, France, from 1985 to 2007 and divided into three groups according to the time interval between prior TIA and stroke ( $<4$ weeks, $\geq 4$ weeks, no TIA) or the duration of TIA ( $\leq 30$ minutes, $>30$ minutes, no TIA). Outcome was dementia diagnosed by neurologists using DSM-III or IV criteria over the first month after stroke. The Chi-squared test was used to compare the prevalence of dementia in each group. Multivariate analyses were performed using logistic regression models.

Results: The prevalence of early dementia was 20.6\% (95\% CI: 18.5-22.7), 26.8\% (95\% CI: 13.3-40.4; p < 0.0001) and $33.1 \%$ 
(95\% CI: 27.3-38.9) among the patients without TIA, with a prestroke TIA $\geq 4$ weeks and with a prestroke TIA $<4$ weeks, respectively. Patients with prestroke TIA $<4$ weeks (adjusted OR: 1.83; 95\% CI: $1.32-2.52 ; \mathrm{p}=0.0003$ ) and $\leq 30$ minutes (adjusted OR: 1.97; 95\% CI: $1.39-2.78 ; \mathrm{p}=0.0001$ ) had a higher risk of early dementia than those without TIA. Interaction terms between age and TIA $(\mathrm{p}=0.024)$.

Conclusion(s): An ischemic stroke is harmful for cognition especially in patients who have had a recent short TIA.

\section{1 \\ Female Reproductive Factors, Postmenopausal Hormone Use, and Parkinson's Disease}

Chen H. ${ }^{\mathrm{a}}$, Liu R. ${ }^{\mathrm{b}}$, Baird D. ${ }^{\mathrm{a}}$, Park Y.c, Freedman N. ${ }^{\mathrm{c}}$, Huang X. ${ }^{\mathrm{d}}$, Hollenbeck A. ${ }^{\mathrm{e}}$, Blair A. ${ }^{\mathrm{c}}$

${ }^{a} \mathrm{NIEHS}$, USA; ${ }^{\mathrm{b}}$ National Institute of Environmental Sciences, USA; ${ }^{C} \mathrm{NCl}$, USA; ${ }^{\mathrm{H}}$ Hersey Medical Center, USA; ${ }^{\mathrm{e}} \mathrm{AARP}, \mathrm{USA}$

Background: Parkinson's disease (PD) is less prevalent in women; the possible influence of hormone-related factors has been of interest, but prior studies have included small samples. We prospectively examined reproductive factors and exogenous hormone use in relation to PD risk among postmenopausal women.

Methods: Participants comprised 119, 166 postmenopausal women ages 50-71 years in the NIH-AARP Diet and Health Study, who completed a baseline questionnaire in 1995-1996 and a follow-up survey in 2004-2006. A total of 410 PD cases were identified between 1995 and 2006. Multivariate odds ratios (OR) and $95 \%$ confidence intervals (CI) were derived from logistic regression models.

Results: Overall, PD risk was not associated with female reproductive factors including age at menarche, age at first live birth, parity, and age at menopause. For example, compared with women with menopause at ages $50-54$ years, the ORs were 1.18 , (95\% CI 0.78-1.79) for women with menopause at ages < 45, 1.19 $(0.88-1.61)$ for menopause at ages $45-49$, and $1.34(0.92-1.94)$ for menopause after age 55 . We found that oral contraceptive use for $\geq 10$ years (vs. never use) was associated with lower PD risk (OR = $0.59 ; 0.38-0.92)$. Replacement hormone use showed inconsistent results. Compared with non-hormone users, current users of $<5$ years showed a higher $\mathrm{PD}$ risk $(\mathrm{OR}=1.52 ; 1.11-2.08)$. However, no associations were observed for past users of $<5$ years or current users of $\geq 5$ years.

Conclusion(s): This large prospective study provides little support for an association between reproductive factors or exogenous hormone use and PD risk.
89

\section{Age and Sex Specific Mortality in Amyotrophic Lateral Sclerosis (ALS)}

Gallo V. ${ }^{\text {a }}$ Brayne C. ${ }^{\mathrm{b}}$, Forsgren L. ${ }^{\mathrm{c}}$, Vermeleun . $^{\mathrm{d}},{ }^{\text {, Kyrozis A. }}{ }^{\mathrm{e}}$, Teucher B. ${ }^{\mathrm{f}}$, Arriola L. ${ }^{\mathrm{g}}$, Erro E. ', Gavrila Chervase D. ', Sacerdote C. ${ }^{\mathrm{j}}$, Sieri S. ${ }^{k}$, Masala G. ', Panico S. ${ }^{m}$, Vanacore N. ${ }^{n}$, Hansson O. ${ }^{\circ}$, Middleton L. ${ }^{\mathrm{p}}$, Riboli E. ${ }^{\mathrm{p}}$

aLondon School of Hygiene and Tropical Medicine, United

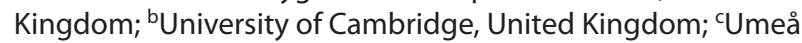
University, Sweden; dUniversity Medical Centre Utrecht, The Netherlands; 'University of Athens, Greece; ${ }^{\mathrm{f}}$ German Cancer Research Centre, Germany; ${ }^{9}$ Public Health Division of Gipuzkoa, Spain; hNavarre Hospital Complex, Spain; 'Murcia Regional Health Council, Spain; 'ंCentre for Cancer Prevention (CPOPiemonte), Italy; ${ }^{k}$ Fondazione IRCCS Istituto Nazionale dei Tumori, Italy; 'Cancer Research and Prevention Institute, Italy;

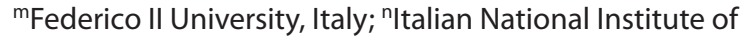
Health, Italy; ${ }^{\circ}$ Skåne University Hospital, Sweden; PImperial College London, United Kingdom

Background: Surprisingly few risk/protective factors have been consistently identified in Parkinson's disease (PD), so far. Prospective cohort studies are the optimal study design in observational epidemiology for causal inference; they are recall biasfree and the potential for selection bias is minimised if the follow-up is complete. Aim of this research is to identify PD cases within the EPIC cohort.

Methods: A total of 299, 568 individuals recruited across 16 EPIC centres were included in the study. Case ascertainment procedures were articulated in 3 phases: phase I - possible cases identification via record linkage and/or self-report; phase II - case validation and collection of additional clinical information; phase III - across centre validation.

Results: A total of 735 PD case were expected on the basis of the age distribution of the populations. During phase I, 1, 349 possible cases were identified. Of these, 555 cases were reviewed in 10 out of 16 centres. Among reviewed cases, 355 PD cases were identified, in addition to 16 multiple system atrophy, 9 progressive supranuclear palsy, 12 vascular parkinsonisms, 16 Lewy body dementia, 15 essential tremor, and $4 \mathrm{PD} /$ essential tremor cases. All cases were either labelled as definite, very likely, probable or possible on the basis of the quality and amount of clinical information available, and the degree of confidence of the reviewing neurologist. The algorithm will be further revised in phase III.

Conclusion(s): The present study represents a proof-of-principle of methods for ascertaining PD cases in large populationbased studies. 
92

\section{Care Management for ALS Patients}

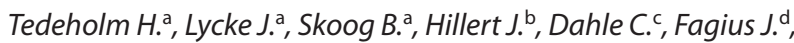
Fredrikson S. ${ }^{b}$, Landtblom A.M. ${ }^{c}$, Malmestrom C. ${ }^{a}$, Martin C. ${ }^{e}$, PiehI F. ${ }^{\mathrm{b}}$, Runmarker B. ${ }^{\mathrm{a}}$, Stawiarz L. ${ }^{\mathrm{b}}$, Vrethem M. ${ }^{\mathrm{c}}$, Nerman O.f, Andersen $\mathrm{O}^{\mathrm{a}}$

${ }^{a}$ Neuroscience and Physiology, Sweden; ${ }^{b}$ Clinical Neuroscience, Sweden; 'Clinical and Experimental Medicine, Sweden;

${ }^{\mathrm{d}}$ Neurology, Sweden; ${ }^{\mathrm{e} C l i n i c a l}$ Science, Sweden; ${ }^{\mathrm{f}}$ Mathematical Statistics, Sweden

Background: Several surveys reported decreasing disability in current multiple sclerosis patients treated by immunomodulatory drugs. Yet it is not clear whether this is a change of the long-term evolution (trajectory) of the disease, or whether it depends on identification of an increased number of mild cases. The objective is to facilitate the use of historical controls in a study of long-term effects of treatment with first generation immunomodulatory drugs (IFN beta or glatiramer acetate).

Methods: A "contemporary" material of patients treated with first generation immunomodulators (onset 1995-2004, $\mathrm{N}=730$ ) and a "historical" incidence cohort (onset 1950-64, $\mathrm{N}=186$ ) were combined in a survival analysis with secondary progression as outcome. Predictive characteristics at onset were used 1) to adjust for different severity and 2) to reveal selection of mild cases in the contemporary group.

Results: The adjusted hazard ratio of secondary progression was $1.69(\mathrm{p}<0.001)$ in the historical material compared to the contemporary. A higher proportion of mild cases was identified in the contemporary material.

Conclusion: The trajectory of MS has changed in the patients treated by first generation DMD, with $41 \%$ lower hazard of secondary progression. It may be possible to further develop the method of historical controls in national registers.

94

Prognostic Factors for Medically Intractable Epilepsy: A Systematic Review

Wassenaar $M$.

UMC Utrecht, The Netherlands

Background: One third of all epilepsy patients have medically intractable epilepsy. Knowledge of prognostic factors that, in an early therapeutic stage of epilepsy, herald intractability could facilitate patient management. In this systematic review, we examined the evidence for independent prognostic factors of intractability in patients with epilepsy.

Methods: MEDLINE and EMBASE were searched for cohort studies reporting on prognostic factors for medically intractable epilepsy. After selection of abstracts, full-text articles were obtained and their quality was assessed by two reviewers, using the QUIPS checklist. All independent prognostic factors in the individual studies were summarized.
Results: Eleven cohort studies were included, of which ten hospital-based. Younger age at seizure onset, symptomatic etiology, high initial seizure frequency, medical history, epileptic EEG abnormalities, and failure of previous antiepileptic-drugs (AEDs) were documented as independent prognostic factors of intractability in at least 2 of the 11 studies; none of these factors was reported in all 11 studies. None of the studies considered genetic, neurobiological, or immunological factors. The studies were of moderate quality, mostly because they did not provide a conceptual model for the choice of predictors. Heterogeneity in study design, population, candidate prognostic factors, and outcome definitions precluded statistical pooling.

Conclusion: While potentially relevant prognosticators of medially intractable epilepsy have been identified, the evidence for these factors is not consistent. There is a need for well-designed prognostic population-based cohort studies that also include pharmacological, genetic, neurobiological, and immunological factors. A valid model for the early prediction of medically intractable epilepsy could improve patient management.

\section{5}

\section{The British Neurodegenerative Outcome Working Group}

Gallo V.a , Brayne C. ${ }^{\text {, }}$ Blossom S. , Barker R. ${ }^{b}$, Shlomo Yoav B. ${ }^{\text {, }}$ Beral V. , Canoy D. ${ }^{e}$, Doyle P. ${ }^{\mathrm{e}}$, Gallacher J. ${ }^{f}$, Green J. ${ }^{\mathrm{e}}$, Ishihara L. ${ }^{\mathrm{g}}$, Sudlow $C$.

aLondon School of Hygiene and Tropical Medicine, United Kingdom; ' University of Cambridge, United Kingdom; 'Newcastle University, United Kingdom; ${ }^{\mathrm{d} B r i s t o l}$ University, United Kingdom; ${ }^{\mathrm{e} O x f o r d ~ U n i v e r s i t y, ~ U n i t e d ~ K i n g d o m ; ~}{ }^{\mathrm{f} C a r d i f f}$ University, United Kingdom; ${ }^{9}$ Glaxo-Smith Kline, United Kingdom; ' 'University of Edinburgh, United Kingdom

Background: Neurodegenerative diseases are relatively neglected in epidemiological research. Most are rare (except for dementia) and their incidence increases with increasing age. Very little is known in terms of their risk factors. Information generated by large, well-established, prospective population-based studies is of paramount importance for defining a risk profile for these conditions.

Methods: A Neurodegenerative Outcome Working Group (NOWG) was set up in 2011 to discuss the development of guidance for ascertaining neurodegenerative conditions within large longitudinal studies and promote the development of internationally recognised guidelines. The focus of the NOWG is to analyse strengths and limitations of different ascertainment methods. Of particular interest is the suitability of different methods for different conditions, and how this might vary according to context.

Results: Guidance on options for the identification of Amyotrophic Lateral Sclerosis (ALS) cases is being produced, mainly based on the experience gathered in European Prospective Investigation into Cancer and Nutrition (EPIC) and in the Million Women Study. Guidelines on options for the ascertainment of Parkinson's disease (PD) cases are currently under study, and the guidelines will be enriched by insights obtained from validation of 
the diagnosis of PD in the UK BioBank cohort. Dementia case ascertainment is the most challenging task, and will be the focus of later stages of the NOWG activities.

Conclusion(s): The NOWG will produce toolkits for evaluating costs and benefits of different strategies for ascertaining neurodegenerative diseases in large population-based studies.

\section{8}

\section{The ALS Register Swabia - Epidemiology and Risk Factors of ALS in Southern Germany}

Nagel G., Ludolph A., Ünal H., Rothenbacher D., Rosenbohm A.

Ulm University, Germany

Background: Rare diseases are increasing recognized as important medical and social issue. Amyotrophic lateralscerlosis (ALS) is the most prevalent of the motorneuron diseases. ALS is characterized by rapidly progressive damage to the motor neurons with survival of 3-5 years. Population-based registries are important elements in the etiological research of ALS.

Methods: Our aim was to implement the first clinical-epidemiological ALS registry in order to estimate (crude and according to EU-Standard population age-standardized) ALS incidence to investigate risk factors in a register-based case-control study.

Results: The ALS registry Swabia started in October 2010 with both the retrospective $(1.10 .2008-30.09 .2010)$ and prospective collection (from 1.10.2010) of ALS cases in a target population of 8.6 million in Southern Germany. In the retrospective data, 419 cases 216 men and 194 women have been identified. On average, the age at diagnosis was 64 (SD12) years. Most ALS cases occurred in the age group over 75 years. The crude incidence was 2.4 and the age-standardized incidence rate was 2.1 (95\% CI 1.9-2.3) per 100,000 person-years. Prospectively data of 152 patients were collected, of which about $75 \%$ agreed to participate in a populationbased case-control study. All participants in case- control study provided a blood sample.

Conclusion(s): The ALS registry Swabia has been implemented successfully. The in Swabia observed incidence rates are comparable with other European data which ranged between 1.5 and 2.7 (Longroscino et al. 2010). The comparison with a control group and the analyses parameters in the blood sample will facilitate to identify risk factors and to explore the underlying pathophysiological mechanisms.

\section{3}

\section{Prevalence of Dementia in Québec, Canada 1999- 2010: An Observational Study Based on Administrative Data}

\author{
Bocti C. ${ }^{\mathrm{a}}$, Rochette L. ${ }^{\mathrm{b}}$, Emond V. ${ }^{\mathrm{b}}$, Kroger E. ${ }^{\mathrm{c}}$ \\ aResearch Centre on Aging, Canada; ' Institut National de Santé \\ Publique du Québec, Canada; 'CEVQ, Hôpital St-Sacrement, \\ Canada
}

Background: In Canada, no estimates of the prevalence of dementia have been published since the landmark Canadian Study of Health and Aging in 1994. Our objective was to estimate prevalence of dementia over a 12-year period (1999-2010) for people 65 years and older using administrative data.

Methods: This population-based study used data from a linked medico-administrative database in the province of Québec, Canada, including an admissibility registry, outpatient medical services, pharmaceutical prescription, hospital-based services and death registry. Dementia cases were identified using three algorithms with different combinations of ICD dementia-related diagnostic codes from physician claims/hospital discharge, with or without prescription claims for 4 approved dementia medications. Prevalence was age-standardized for the study of trend between 1999 and 2010.

Results: There were about 1.1 million individuals included in this study. In 2009-2010, between 5.5\% and 8.8\% were identified by the three different case definitions. Physician claims was the main data source for case ascertainment followed by prescription data. About two-thirds of cases were women. Prevalence rises steadily but exponentially in older age groups. In 2009-2010, about $1 \%$ of persons aged 65 to 69 years were identified by the algorithms; estimates varied from $27 \%$ to $38 \%$ for people 90 years and older. Preliminary trend analyses indicate a relative increase of about 30\% in the prevalence of dementia from 1999 to 2006 followed by stabilization until 2010. This plateau was apparent for the 3 algorithms.

Conclusions: Preliminary results from these case definitons may underestimate prevalence due to possible underreporting in administrative data, especially for older age groups. Further research will validate these estimates. Ongoing surveillance for chronic diseases such as dementia is useful to guide and evaluate decisions about policies, programs, services, education and research.

\section{6 \\ Risk of Grave's Disease Among MS Patients on Disease Modifying Drugs}

Svenson L., Schopflocher D., Warren S.

University of Alberta, Canada

Background: The objective of this study was to determine if multiple sclerosis (MS) patients on disease modifying therapies had an increased risk of developing Grave's disease. 
Methods: The province of Alberta maintains a publicly funded universally available health care system. The Ministry of Health maintains linkable population-based data that includes a population registry, physician services, hospital visits, emergency department visits, and drug plan data.

Individuals were classified as having MS if they had a least two physician visits (ICD-9 code 340) in a two year period or at least one hospitalization (ICD-10 code G35). This definition has a specificity of $99.7 \%$ and sensitivity of $73.4 \%$. All individuals receiving a MS disease modifying drug were extracted from the pharmacy drug database for the period 2006 to 2011. To determine Grave's disease status, individuals with an ICD-9 code of 242.0 was extracted. The analysis was limited to those individuals present in the health insurance registry in 2011. Multiple logisitic regression was used to assess the odds of Grave's given the use of MS drugs.

Results: There were 9, 722 individuals over the age of 20 years with MS for a crude prevalence of 340 per 100, 000. 2, 811 (28.9\%) were on an MS drug therapy. There were 56 patients (2\%) on drug therapy with Grave's. Females were more likely to be diagnosed $(\mathrm{OR}=3.6,95 \% \mathrm{CI}: 3.5,3.7)$ and those over age 50 years $(\mathrm{OR}=1.3$, $95 \%$ CI: $1.3,1.4)$. Those receiving MS drugs did not have a higher odds of being diagnosed with Grave's disease $(\mathrm{OR}=1.2,95 \% \mathrm{CI}$ : $0.8,1.7)$.

Conclusion(s): The use of MS drugs does not appear to elevate the risk of developing Grave's disease.

\section{6 \\ Natural History of Unruptured Intracranial Aneurysms}

Juvela S. ${ }^{\mathrm{a}}$ Poussa K. ${ }^{\mathrm{b}}$, Lehto H. ${ }^{\mathrm{b}}$, Porras M. ${ }^{\mathrm{b}}$

aUniversity of Helsinki, Finland; ${ }^{b}$ Helsinki University Central Hospital, Finland

Background: To investigate the long-term natural history of unruptured intracranial aneurysms in a patient population without surgical selection of cases.

Methods: A total of 142 patients with 181 unruptured aneurysms diagnosed between 1956 and 1978 were followed until death or subarachnoid hemorrhage, or until 2011. Incidences of aneurysm rupture as well as risk factors for the rupture were studied with Kaplan-Meier survival analysis and the Cox proportional hazards regression models.

Results: Median follow-up time was 21.0 (mean 21.5, range 0.8 to 52.3 ) years. During 3048 person-years of follow-up, there were 34 first episodes of hemorrhage from a previously unruptured aneurysm, giving an average annual incidence of $1.1 \%$. The cumulative rate of bleeding was $10.5 \%$ (95\% confidence interval, $5.2 \%-15.8 \%)$ at 10 years, and $30.1 \%(21.3 \%-38.9 \%)$ from 30 to 50 years after diagnosis. None of index aneurysms bled after a followup of 25 years. In multivariable analysis, current smoking at the time of diagnosis (adjusted hazard ratio [HR] 2.44, 95\% CI 1.02$5.88, \mathrm{p}<0.05)$, location of aneurysm in anterior communicating artery $(3.73,1.23-11.36, \mathrm{p}<0.05)$, young patient age $(0.96$ per year, $0.92-1.00, \mathrm{p}<0.05)$, diameter of aneurysm ( 1.11 per $\mathrm{mm}$ in diameter, $0.99-1.25, \mathrm{p}=0.088$ ) predicted aneurysm rupture after adjustment for sex, history of hypertension, and aneurysm group. In univariable analysis only, alcohol consumption (1.27 per $100 \mathrm{~g} /$ week, 1.05-1.53, p < 0.05) also predicted an aneurysm rupture.

Conclusion(s): Cigarette smoking, patient age, size and location of unruptured intracranial aneurysm, and possibly alcohol consumption are factors determining risk for subsequent aneurysm rupture.

\section{9
Structural Changes of the Corpus Callosum in Study Cognitive Decline and Dementia: A 7-Year Follow-Up}

Artero S. ${ }^{\mathrm{a}}$, Mortamais M. ${ }^{\mathrm{a}}$, Poulain V. ${ }^{\mathrm{a}}$, Maller J. ${ }^{\mathrm{b}}$, Meslin C. ${ }^{\mathrm{c}}$, Le Bars E. ${ }^{\mathrm{d}}$, Bonafé A. ${ }^{\mathrm{d}}$, Touchon J. ${ }^{\mathrm{d}}$, Ritchie K. ${ }^{\mathrm{d}}$

aINSERM U1061, Montpellier, France; b Monash Alfred Psychiatry Research Centre, The Alfred \& Monash University School of Psychology and Psychiatry, Australia; 'Centre for Mental Health Research, Australian National University, Australia; ${ }^{\mathrm{d}} \mathrm{CHU}$ Montpellier, France

Background: Numerous studies have documented white matter (WM) pathology associated with Alzheimer's disease (AD). The corpus callosum (CC) is the largest WM fiber bundle in the human brain, it has been shown to be particularly susceptible to atrophy in Alzheimer's disease. Few studies, however, have assessed the long-term impact of CC size on cognition. The aim of this 7-year follow up study was to investigate whether CC atrophy may predict cognitive decline and dementia in the healthy elderly population.

Methods: We studied 607 right-handed individuals without dementia from a cohort of community-dwelling persons aged 65 to 80 years (The ESPRIT study). At baseline, T1-weighted magnetic resonance images were traced to measure the midsagittal areas of the anterior, mid and posterior CC. At 2-year, 4-year and 7-year follow-ups, participants were screened for cognitive decline and dementia (clinical diagnosis). Severity of cognitive decline was defined as none, moderate or severe calculated from neuropsychological test performance change. The relation between CC size and longitudinal change in cognition or dementia was studied using respectively polytomous logistic regression and Cox regression models controlling for potential confounders.

Results: Subjects with severe cognitive decline profile were associated to significantly lower anterior and mid areas (OR Anterior CC 1.91 [1.22-3.00], $p=0.005$; OR Mid CC 1.60 [1.05$2.46], p=0.03)$. Moreover, subjects having lower anterior, mid and post areas were significantly more likely to develop dementia (HR Anterior CC 2.23 [1.37-3.65], $p=0.001$; HR Mid CC 1.85 [1.093.13], $p=0.02$; HR Post CC 1.62 [1.05-2.50], $p=0.03$ ).

Conclusion(s): Our findings emphasize the potential pathological role of the CC in dementia and cognitive decline. Its anterior-posterior fiber caliber gradient can help in understanding the pathophysiological mechanisms underlying white matter changes in cognitive decline. 


\section{0}

\section{Past Exposure to Neuroleptics and Risk of Parkinson's Disease}

Foubert-Samier A. ${ }^{\mathrm{a}}$, Helmer C. ${ }^{\mathrm{a}}$, Perez F. ${ }^{\mathrm{b}}$, Le Goff M. ${ }^{\mathrm{a}}$, Auriacombe S. ${ }^{a}$, Elbaz A. ', Dartigues J.-F. ${ }^{\text {, }}$, Tison F. ${ }^{\text {b }}$

aINSERM Unit 897, France; bUniversity Hospital of Bordeaux, France; 'INSERM Unit 708, France; dUniversity of Bordeaux, France

Background: Neuroleptics and neuroleptic-like drugs are known to induce parkinsonism, wich may reveal underlying Parkinson's disease (PD) in some cases. We assessed the long-term risk of developing $\mathrm{PD}$ after a past exposure to these drugs in a 15-year prospective population-based elderly cohort study.

Methods: We used the Cox proportional-hazards model to assess the relation between past exposure to neuroleptics and the risk of developing incident PD. All incident cases of parkinsonism were identified by standardized procedure and validated by a committe of experts.

Results: Of 2991 subjects followed, 117 developed parkinsonism and 43 developed probable PD during follow-up, of whom $22.2 \%$ and $32.6 \%$ respectively have been exposed to neuroleptics, compared to $16.6 \%$ for subjects without parkinsonism. About a third of subjects presented transient parkinsonism during drug exposure. After adjustment for gender and past occupation, past exposure to neuroleptics was associated with incident PD (relative risk 3.16; 95\% CI 1.65 to 6.04). The relative risk was 3.65 (95\% CI 1.41 to 9.45 ) for benzamides and 2.59 (95\% CI 1.23 to 5.43 ) for phenotiazines. The population-attributable fraction of the risk for developing PD was $8.2 \%$ for benzamides and $12.2 \%$ for phenotiazines.

Conclusion(s): In a French elderly cohort, the risk of probable PD was increased by 3.2-fold after exposure to neuroleptics. This finding pleads for limiting the use of such drugs in elderly people.

\section{3 \\ Wilson Disease in France: Follow-Up of a Cohort of 395 Patients}

\section{Girardot-Tinant N. ${ }^{a}$, Trocello J.M. ${ }^{a}$, Ruano E. ${ }^{\text {, }}$, Pelosse M. ${ }^{\text {, }}$ Woimant $F^{\text {a }}$}

${ }^{a}$ French National Center of Reference for Wilson Disease, France; ${ }^{\text {b}}$ Eurowilson, France

Background: One of the priorities of the French National Reference Center (FNRC) for Wilson Disease (WD), labeled end of 2005, is to improve the epidemiological knowledge of this rare disease.

The objective of database for WD is to better define different profiles likely to evoke the diagnosis and to learn more about the disease evolution.

Methods: Demographical, clinical, biological, radiological and therapeutic data of the 395 patients (aged from 1 to 77 years old) in the two reference centres (AP-HP and Lyon) and the 6 competence centres (Besançon, Lille, Aix/Marseille, Toulouse, Bordeaux and Rennes) of the FNRC for WD have been collected at time of diagnosis and/or during follow up.

Results: The diagnosis has been set up most of the time after neurological symptoms (33\%), mean age of 23 years old, or hepatic symptoms $(34 \%)$ or with familial screening $(20 \%)$. The genetic investigation was not significant for $16 \%$ of the families. D-Penicillamine has been the initial treatment for $85 \%$ of the patients and still prescribed after 15 years of follow up for $44 \%$ of the cases. The mean causes of death are hepatic failure, infections and hepatocellular carcinoma.

Conclusion(s): As far as we know, this is the most important cohort of WD patients. However, it covers only about $1 / 3$ of the French patients. In order to improve the registration of the WD patients in the database either at time of diagnosis or during follow up, the coordination of all healthcare professionals with a multidisciplinary approach must be encouraged.

The French registry contributes greatly to Eurowilson database due to the number of patients included and followed.

\section{5 \\ Serum Uric Acid and Risk of Parkinson's Disease in a Prospective Study}

Van Den Eeden S., Albers K., Shan J., Quesenberry C.

Kaiser Permanente, USA

Background: Urate levels have been associated with risk of PD. The objective of this study was to examine the risk of Parkinson's disease (PD) associated with prediagnostic serum uric acid levels in a prospective cohort study.

Methods: (We studied a cohort of individuals who were members of Kaiser Permanente Northern California (KPNC) after 1995 and had participated in Multiphasic Health Checkup (MHC) Cohort exam in 1964-1973 $(n=66,438)$. Cases were determined by review of KPNC electronic clinical records for neurologist diagnosed PD ( $\mathrm{n}=1,154)$. Serum uric acid levels were determined at baseline questionnaire on the entire cohort. Covariate data on age, sex, race/ethnicity, smoking and BMI were also obtained from the MHC. Cox proportional hazard models were used to estimate the hazard ratio (HR) and 95\% confidence inter$\operatorname{val}(95 \%$ CI) for PD associated with serum uric acid level.

Results: The mean follow-up time for the full cohort was 38.8 years and included over 2, 575, 000 person-years of follow-up. Cohort members had a mean age at baseline of 36 years, while the cases were approximately 70 years old at diagnosis. Overall we found no association between serum uric acid levels and PD (HR $=0.95,95 \%$ CI $0.75-1.21$ ). However, when stratified by sex, there was no association in men and an inverse association among women.

Conclusion(s): These prospective data suggest that serum uric acid levels antecedent to Parkinson's disease onset is associated in women, but not men. 


\section{8 \\ Comparison of Coronary Heart Disease and Stroke Risks Attributable to Vascular Risk Factors: Results from the PRIME Study}

Bodenant M. ${ }^{\mathrm{a}}$, Dallongeville J. ${ }^{\mathrm{b}}$, Arveiler D. ${ }^{\mathrm{c}}$, Kee $\mathrm{F}^{\mathrm{d}}{ }^{\mathrm{d}}$, Montaye M. ${ }^{\mathrm{b}}$, Ruidavets J.-B. ${ }^{\mathrm{e}}$, Wagner A. ${ }^{\mathrm{C}}$, Evans $A .{ }^{\mathrm{d}}$, Ducimetière P. ${ }^{\mathrm{f}}$, Ferrières J. ${ }^{9}$

${ }^{a} \mathrm{CHRU}$ Lille, Roger Salengro Hospital; Institut Pasteur de Lille; U744 INSERM; Université Lille Nord de France, Lille, France; 'Department of Epidemiology and Public Health, EA 3430, Université de Strasbourg; Hôpitaux Universitaires de Strasbourg, Strasbourg, France; dUKCRC Centre of Excellence for Public Health (NI), Queen's University, Belfast, United Kingdom; eDepartment of Epidemiology, Centre Hospitalier Universitaire (CHU) de Toulouse, Toulouse, France; ${ }^{f} U M R 780$ INSERM - Université Paris Sud XI, Villejuif, France; ${ }^{9}$ UMR1027 INSERM - Université Toulouse 3, Toulouse, France

Background: Coronary heart disease (CHD) and stroke are the leading causes of death worldwide, although these diseases are preventable. In order to set priorities for public health policies, we sought to assess and compare within a single cohort, CHD and stroke population-attributable risks (PARs) for various cardiovascular risk factors.

Methods: The PRIME study is a multicenter prospective population-based cohort of men living in France or Northern Ireland, aged 50-59 in 1991-93, and followed over 10 years to record CHD (coronary death, myocardial infarction, unstable angina) and stroke.

Results: The sample comprised 9701 men, free of CHD and stroke at baseline. During the follow-up, 410 and 118 cases of CHD and stroke occurred. After adjustment for age, centre, antihypertensive and lipid-lowering treatments, alcohol consumption and other significant cardiovascular risk factors, PARs for CHD were $21.1 \%$ (high blood pressure), $14.8 \%$ (smoking), $11.1 \%$ (hypercholesterolemia), 9.8\% (low HDL-cholesterol), 5.4\% (obesity) and $1.9 \%$ (diabetes). The overall PAR for CHD, referring to the presence of at least one of the above detailed risk factors reached $71.2 \%(79.1 \%$ after exclusion of unstable angina). Adjusted PARs for stroke were 32.8\% (high blood pressure), $15.9 \%$ (smoking) and $6.4 \%$ (diabetes), with no significant impact of the other risk factors. The overall PAR for stroke was $43.7 \%$ (44.6\% for ischemic stroke).

Conclusion(s): The impact of traditional cardiovascular risk factors on CHD and stroke are different. As prevention should primarily focus on factors exhibiting high PARs for both CHD and stroke, high blood pressure and smoking are the best targets for prevention.

\section{2}

Autoimmune Thyroiditis as a Risk Factor for Stroke: A Historical Cohort Study Using the Health Improvement Network Database (THIN)

Karch A. ${ }^{a}$, Thomas S. ${ }^{b}$

aUniversity hospital Goettingen, Germany; ${ }^{b}$ London School of Hygiene and Tropical Medicine, United Kingdom

Background: Autoimmune thyroiditis (AIT) is one of the most frequent autoimmune diseases and the most common cause of hypothyroidism in central Europe. Hypothyroidism is associated with an increased risk for $\mathrm{CHD}$; evidence for an association between hypothyroidism or AIT and stroke is inconclusive. The primary objective of this study was to assess the effect of AIT on stroke. It was also investigated whether the effect varied by age or by AIT duration.

Methods: This was a historical cohort study using data from THIN. Rates of first stroke during follow-up in thyroxine-treated AIT patients ( $\mathrm{n}=64975)$ were compared to those in matched (by age, sex, practice) individuals without AIT $(n=270373)$ using random-effects Poisson regression models. A sub-cohort restricted to incident AIT cases and their matched unexposed was used to investigate possible causal pathways to stroke and effect modification by age and AIT duration.

Results: Initial analysis of the entire cohort showed strong evidence for a slightly increased risk of stroke in AIT patients (RR: 1.09, 95\% CI: 1.03-1.15). In the subsequent analysis restricted to incident AIT cases and their matched unexposed, similar RRs were observed, varying slightly depending on a-priori assumptions. Higher effect sizes were identified in the first year after AIT diagnosis, but not in the long-term.

Conclusion(s): Results of this study support the hypothesis of a slightly increased risk of stroke in AIT patients compared to individuals without AIT. High effect sizes in the first year after diagnosis are compatible with an increased risk due to thyroid-hormone deficiency, but not due to autoimmune processes. Better screening and early treatment of asymptomatic hypothyroid AIT patients should be considered to help reduce excess risk of stroke in the first year after diagnosis.

\section{9 \\ Risk Factors for Incident Dementia and Alzheimer Disease in the NEDICES Cohort}

Bermejo-Pareja F. ${ }^{\mathrm{a}}$, Trincado R. ${ }^{\mathrm{b}}$, Moreno T. ${ }^{\mathrm{c}}$, Sambricio J. ${ }^{\mathrm{b}}$, Villarejo A. ${ }^{\mathrm{b}}$, Vega S. ${ }^{\mathrm{d}}$, Benito-León J. ${ }^{\mathrm{b}}$

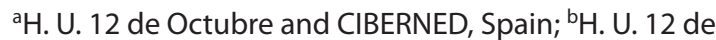
Octubre, Spain; 'Neurology Department, University Hospital "Clínico de San Carlos", Madrid, Spain; 'ARévalo Health Centre, Spain

Background: The scarce success in the treatment of established dementia promotes the risk factors (RF) study to undertake preventive measures. The objective is this work is to analyse the 
RF for dementia and Alzheimer disease (AD) in the NEDICES (Neurological Disorders in Central Spain) elderly cohort.

Methods: 5, 278 census-based elderly participants were evaluated at baseline (1994-1995) and at follow-up (a median of 3.2 years later in 1997-1998). At baseline a long questionnaire (500 items) about health status and life style variables were completed in the majority of participants.

Results: 161 incident dementia cases were identified among 3, 891 individuals assessed at follow-up. The large majority had AD: 115 (71.4\%) (J Neurol Sci, 2008, 264:63-72). In several Cox regression analyses, age, education (to be illiterate), previous cognitive performance (MMSE-37), to suffer a previous stroke (and to have more than four vascular RF) were the main consistent, statistically significant and biological plausible $\mathrm{RF}$ for dementia and $\mathrm{AD}$. Others less consistent RF were: low physical activity (and functional disability), subjective health status, and excessive sleeping behavior. Moderate alcohol intake was slightly associated with less dementia incidence. The RF statistical analysis description is too long to be described in the abstract.

Conclusion(s): NEDICES data suggest, like other observational cohorts, that the reduction of vascular risk factors and an increase in physical activity in the elderly must be tested in dementia and $\mathrm{AD}$ preventive trials.

\section{6}

\section{Is Maternal Influenza Associated with Autism?}

Svenson L., Schopflocher D.

University of Alberta, Canada

Background: It has been suggested that fever during pregnancy may increase the risk of developing autism among offspring. The purpose of this study was to assess the risk of being diagnosed with autism among children whose mothers had been diagnosed with influenza during pregnancy using populationlevel data.

Methods: The province of Alberta maintains a publicly funded, universally available health care system. As part of managing this system, a unique lifetime identifier is provided to all residents and is captured for all health services allowing linkages to be done. A cohort of 48, 813 births occuring in 2007 were extracted from the Newborn Metabolic Screening program. These data were linked to the Alberta Vital Statistics birth registration system for both maternal and newborn characteristics. Linkage was also done with the physician fee-for-service (FFS) data, from 2006 and 2007, to determine if the mother had been diagnosed with influenza (ICD-9 487) during pregnancy. The FFS data were also used to identify who had been diagnosed with autism (ICD-9 299.0) between 2007 and 2011.

Results: Among the 48, 813 (24, 025 Female; 24, 788 Male) children in the cohort, 227 were diagnosed with autism within the first four years of live for a birth cohort prevalence of 4.7 per 1, 000 live births. Males were more likely to be diagnosed with autism (OR $=4.70,95 \% \mathrm{CI}: 3.33,6.64)$. Among the children diagnosed with autism, 6 (2.6\%) of the mothers had been diagnosed with influenza, compared to $2.2 \%$ of women without having a child diagnosed with autism ( $\mathrm{p}>.05)$. Overall, 6.69\% of the cohort were low birth weight. For those diagnosed with autism, 12.1\% were low birth weight. Statistically significant odds ratios were found for low birth weight $(\mathrm{OR}=1.72 ; 95 \% \mathrm{CI}: 1.21,2.46)$ and maternal age between 40 and $44(\mathrm{OR}=2.54,95 \% \mathrm{CI}: 1.25,5.17)$ or maternal age greater than 45 years $(\mathrm{OR}=12.27,95 \% \mathrm{CI}: 2.83$, 53.18).

Conclusion(s): A diagnosis of influenza during pregnancy does not appear to be associated with an increased likelihood of autism. Older maternal age and low birth weight were associated with an increased likelihood of autism being diagnosed among off-spring.

\section{5}

\section{ARCOS IV: Stroke Incidence and Outcomes}

\section{Krishnamurthi R., on behalf of ARCOS IV Programme Collaborators}

National Institute for Stroke and Applied Neurosciences, AUT University, Auckland, New Zealand

Background: Stroke is the 2nd most common cause of death worldwide and a frequent cause of adult disability in developed countries. ARCOS IV is a population-based stroke/TIA incidence and outcomes study, with case ascertainment from the geographical boundaries of the Greater Auckland Region, New Zealand. The aim of the Auckland Regional Community Stroke Study (ARCOS IV) is to measure and reduce stroke burden in New Zealand. The study is conducted at the National Institute for Stroke and Applied Neurosciences, AUT University, and preliminary findings are presented on behalf of the ARCOS IV Programme Group.

Methods: The "hot-pursuit" method of case ascertainment is being used to register all first-ever and recurrent stroke and transient ischaemic attack (TIA) cases (both hospitalised and nonhospitalised, fatal and non-fatal) from 1st March 2011 to 29th February 2012 using multiple overlapping sources of information. Participants consented into the study are followed up at baseline, 1, 6 and 12 months post-stroke (in this section, design, subjects, and statistical analysis used in the study are briefly described).

Results: To date, there were just over 2200 incident cases of stroke and TIA. Cross-checking with official databases, including mortality data, is underway. Approximately $98 \%$ of cases presented at a hospital and $2 \%$ were from rest homes or visited their GP only. Males represented $48 \%$ of strokes, and $48 \%$ of strokes were in the $\geq 75$ years age group with $3 \%$ being $\leq 40$ years. NZ Europeans represented $57 \%$ of strokes and TIAs, Maori 6\%, and Pacific Islanders $11 \%$. Approximately $55 \%$ of participants consented to follow-up, due to to completed by 28th February, 2013.

Conclusion(s): ARCOS recruitment is now complete and completion of follow-up, cross-checking for complete case ascertainment, and data cleaning is underway. Final reports of stroke/TIA incidence and outcomes, including case-fatalities are expected to be available in the next 1-2 years. (in this section, importance of results of the study for practice and/or future research is briefly described). 
197

Epidemiology of Epilepsy Among Elderly People in Al Quseir City, Egypt

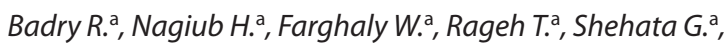
Badry N. ${ }^{\mathrm{b}}$, Abd Al Wareth A. ${ }^{\mathrm{a}}$, Kandil M. ${ }^{\mathrm{a}}$

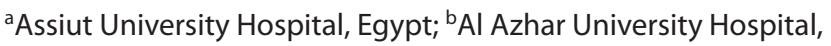
Egypt

Background: Older people face special challenges in living with and adjusting to epilepsy and seizures. Epilepsy in elderly people is one of the underestimated health problems in Egypt. Objectives: To estimate the prevalence of epilepsy in elderly people in Al Quseir city- Red Sea Governate, Egypt and to estimate its magnitude of treatment gap.

Methods: The study was a part of door to door study, including every door to screen all inhabitants in Al-Quseir city $(33,818$ inhabitants); by 3 specialists of neurology and 15 female social workers. Number of people $\geq 60$ years is 2106 persons among whom we detected 13 cases with epilepsy. All suspected cases were subjected to detailed history, clinical examination, EEG, Neuroimaging studies.

Results: The study revealed that life time prevalence of epilepsy is $6.2 / 1000$, with highest peak during the eighth decade of life, while the prevalence of active epilepsy is $2.4 / 1000$ population. The prevalence rate of epilepsy in elderly males is double that in elderly females. Epilepsy in elderly detected in this study is caused by symptomatic cause $(30 \%, \mathrm{n}=4)$, idiopathic $(53.8 \%, \mathrm{n}=7)$, and cryptogenic $(15.4 \%, \mathrm{n}=2)$. The annual incidence rate is $142.4 / 100$, 000 . Focal epilepsy is the most frequently encountered type $(84.6 \%)$. The treatment gap of epilepsy in elderly in Al Quseir city is $80 \%$.

Conclusion: Epilepsy among elderly is an underestimated health problem in Egypt and treatment gap is high which necessitates developing health services in this locality.

\section{7 \\ The Incidence and In-Hospital Case Fatality of First- Ever Stroke in the Northern Aegean Island of Lesvos, Greece

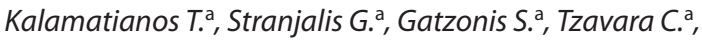 Loufardaki M. b', Sakas D. ${ }^{\text {a }}$, Group Lesvos \\ anniversity of Athens, Greece; ${ }^{b}$ Hellenic Centre of Neurosurgical Research, Professor Petros S. Kokkalis, Greece}

Background: There is a paucity of research on the incidence and distribution of stroke types in Greece. This is the first study investigating the incidence of stroke in a Greek island, namely the Northern Aegean island of Lesvos (population 90643).

Methods: A multisource prospective population-based register was established and all subjects in the study area with first-ever stroke (FES) between June 1st 2010 and May 31st 2011 were identified.

Results: During the 12-month study period, 197 subjects with FES were registered, 112 males and 85 females, with a mean age $( \pm$
SD) of $75 \pm 12$ years. In men, cerebral infarction was diagnosed in $78.6 \%$ of cases, intracerebral hemorrhage in $20.5 \%$, and subarachnoid hemorrhage in $0.9 \%$, while in women the respective figures were $94.1 \%, 2.4 \%$ and $3.5 \%$. The incidence rates (per 100000 ) by age group ( 45 to 54,55 to 64,65 to 74,75 to $84,>/=85$ years) for men were $95,365,594,1396$, and 2528 , respectively. For women, these rates were 131, 169, 243, 941, and 1871, respectively. Ageand sex- standardized to the European population, the annual incidence rate of stroke for subjects aged 45 to 84 years was $303.9 / 100000$ (95\% CI, 254 to 360 ). In-hospital 30 day case fatality for the total sample was $13.5 \%$ (13.8\% for men and $13.3 \%$ for women).

Conclusion(s): The present findings indicate that the incidence of stroke in the studied population is comparable to that estimated previously by several population based studies in the Mediterranean area.

This study was supported by an unrestricted research grant by Sanofi, Greece.

\section{0}

\section{Cancer and Alzheimer Disease: An Inverse Relationship of Occurrence. Results from a Population Italian Study}

\author{
Musicco M. ${ }^{a}$, Prinelli F. ${ }^{a}$, Russo A. ${ }^{b}$, Adorni F. ${ }^{\mathrm{a}}$

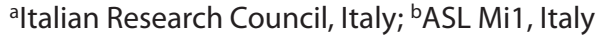

Background: Alzheimer's disease (AD) and cancer increase their frequency with age and can be considered main negative consequences of senescence. Few previous studies reported a lower incidence of cancers in persons with Alzheimer's disease (AD) and lower incidence of AD in persons with cancers. This result needs to be confirmed in different sets and with different study designs since it might be due to confounding due to underreporting and/or shortening of life expectancy of persons with cancer or $\mathrm{AD}$.

Methods: This was a prospective/retrospective cohort study. Persons living in the geographical area of the local health authority (ASL) of Milano 1 (Mi1) of 60 or more year from 2004 to 2009 with a first diagnosis of AD or cancer were recruited in their specific diagnostic cohort. Diagnoses were retrieved from the ASL Mil informative health system. Based on the person-years of observation during the time period from 2004 to 2009 we calculated the incidences of $\mathrm{AD}$ in the cohort of cancers and the incidence of cancers in the $\mathrm{AD}$ cohort in the period preceding and following the index diagnosis. The incidence of $\mathrm{AD}$ and cancers were calculated also for the general population of ASL Mil and observed cases in the two cohorts with respect to those expected from the general population were compared. Separate comparisons were made for the period preceding and following the diagnosis of $\mathrm{AD}$ or Cancer, for $\mathrm{AD}$ occurrence in smoking and non smoking related cancers and for persons surviving or dying during the period 2004-2009.

Results: In the whole population of ASL Mi1 we registered 21451 (12225 men) newly diagnosed cancers and 2832 AD (947 men) giving respectively a crude incidence rate per 10000 p-y of 
Table for Abstract 224 Digit-Symbol Substitution Test scores, by saturated fat intake and statin use*

\begin{tabular}{|c|c|c|c|c|c|c|c|}
\hline \multicolumn{2}{|c|}{ Saturated fat intake } & \multicolumn{2}{|l|}{ Statin user } & \multicolumn{2}{|l|}{ Non-user } & \multicolumn{2}{|c|}{$\mathrm{P}$-value } \\
\hline (\% or energy) & $\mathrm{n}$ & mean $+/-S E$ & $\mathrm{n}$ & mean $+/-\mathrm{SE}$ & difference & diff & interaction \\
\hline$>/=10$ & 201 & $46.58+/-0.91$ & 932 & $49.23+/-0.69$ & -2.65 & 0.035 & 0.002 \\
\hline$<10$ & 228 & $50.09+/-0.95$ & 845 & $46.89+/-0.67$ & 3.20 & 0.004 & \\
\hline
\end{tabular}

${ }^{*}$ Results are controlled for age, gender, race-ethnicity, years of education, history of smoking and alcohol use, diabetes status, fish intake, exercise status (i.e., did or did not engage in vigorous aerobic activity; did or did not engage in moderate aerobic activity), and serum concentrations of folate, vitamin B-12, methylmalonic acid, homocysteine, creatinine, alkaline phosphatase, and globulin.

175.1 and of 22.1. The cohort of persons with AD contributed a total of $15063.0 \mathrm{p}$-y of observation and the corresponding figure for the cancer cohort was 101317.9 p-y. In 161 cases cancer and $\mathrm{AD}$ were diagnosed to the same person with $\mathrm{AD}$ preceding cancer in 68 cases. In persons with cancer 246 cases of $\mathrm{AD}$ were expected from the incidence observed in the whole population of 60 years or older; the risk of $\mathrm{AD}$ occurrence, relative to that of the general population of the same age and gender was therefore significantly reduced (observed/expected $=0.6595 \%$ Confidence Interval $[\mathrm{CI}]$ 0.56-0.76). The corresponding figure for the cohort of $\mathrm{AD}$ were 241.2 cancer expected with a relative risk (RR) of 0,57 (95\% CI $0.49-0.67)$. No significant differences in the estimates were observed for the follow-up periods preceding and following the diagnosis, for smoking related cancers or for members of the cohort not surviving for the entire period of follow-up.

Conclusion(s): Cancer and $\mathrm{AD}$ are negative manifestation of senescence and have an inverse relationship of occurrence. This result might be interpreted as a confirmation for the theory of pleiotropic genes for aging and senescence.

\section{4}

\section{Interaction Between Statin Use and Saturated Fat Intake in Relation to Cognitive Test Performance}

Morris M., Wang $H_{\text {., }}$ Jacques $P$.

Tufts University, USA

Background: Strokes, microvascular disease, and Alzheimer's disease adversely affect cognitive function in older people. High circulating cholesterol levels and amyloid-beta peptide deposition contribute to these conditions. Statins lower serum cholesterol by interfering with cholesterol biosynthesis, and they may also inhibit amyloid-beta production. Consequently, it is reasonable to hypothesize that statins would help prevent age-related cognitive impairment. However, study results have been mixed, and the US Food and Drug Administration warns of memory loss and confusion as possible consequences of statin use. The circumstances under which statin use might enhance or impair cognitive function deserve attention. Statin users are advised to consume a diet low in saturated fat to obtain maximum benefits. We were interested in potential effect modification by such a diet on the association between statin use and cognitive function.
Methods: Participants aged $>/=60$ years in the US National Health and Nutrition Examination Survey (1999-2002) were administered the Digit-Symbol Substitution Test (DSST) of information processing speed. Dietary data were collected via 24 -hour recall; blood was drawn for the determination of many nutritional and health-status indicators, and information on lifestyle factors and chronic disease history was collected via questionnaire. Multiple linear regression analysis was used to assess the effect of meeting (versus not meeting) the 2010 Dietary Guidelines for Americans recommendation for saturated fat intake on the association between statin use and DSST performance among 2206 subjects who did not report a history of stroke.

Results: After controlling for demographic, nutritional, and lifestyle factors, the mean DSST score for statin users whose saturated fat intakes comprised at least $10 \%$ of their energy intakes was lower than that of non-users who also had high saturated fat intakes $(\beta=-2.65, P=0.035)$ (Table). On the other hand, among subjects who met the current recommendation for saturated fat intake of $<10 \%$ of energy, statin users had higher DSST scores than non-users of statins $(\beta=3.20, \mathrm{P}=0.004$, P-interaction $=$ 0.002).

Conclusion(s): Whether statin use enhances or impairs cognitive function may depend on saturated fat intake.

\section{9}

\section{The Shanghai Parkinson Study: Prospective Parkinson Research in China}

Chen $\mathrm{H}$.

NIEHS, USA

Background: China has the largest population of Parkinson's disease (PD) patients; however few etiological studies have been conducted in China.

Methods: The Shanghai Women's Health Study recruited 74, 941 women in urban Shanghai, aged 40 to 70, from 1996 to 2000. Baseline blood and urine samples were collected from most participants. Self-reported PD cases were invited for a neurological examination and diagnoses were made by movement disorder specialists according to established criteria.

Results: This cohort had very few ever smokers (2.8\%), alcohol drinkers (2.2\%), and post-menopausal hormone users (2.6\%); however, tea drinking (29.8\%) and secondary smoking from hus- 
band (61.7\%) were common. A total of 301 self-reported PD were identified from the third and the fourth follow-up surveys. Of these, 95 PD diagnoses were confirmed via either direct clinical examinations $(n=76)$ or medical history review $(n=19)$. In this cohort, PD risk appears to be inversely associated with secondary smoking from husband and tea drinking, but directly with education. The age adjusted OR was $0.8(95 \% \mathrm{CI}=0.5-1.3)$ for husband ever smoking, $0.6(0.4-1.0)$ for husband smoking at the baseline survey, and $0.8(0.5-1.3)$ for ever tea drinking. Compared with primary education or lower, the age adjusted OR was $1.3(0.7-2.4)$ for middle school education and $1.6(1.0-2.7)$ for high school or above.

Conclusion(s): PD research in this unique cohort is feasible and, with extended follow-up of the cohort, will allow us to conduct prospective etiological research in China.

\section{3 \\ Morbidity and Mortality Trends at 28 Days in First- Ever-Stroke Cases in Mumbai Stroke Registry (2005- 06 and 2009)}

Dalal P., Bhattacharjee M., Vairale J.

LKMM Trust Research Centre, India

Background: India is facing socioeconomic burden on rehabilitation of stroke survivors.

Methods: A well-defined population in H-district Mumbai with verifiable census data was selected. 156, 861 persons between the age of 25 and 94+ years were screened in Study I(2005-06) and 174,398 persons from the same population were screened in Study II (2009). WHO STEPwise approach to stroke surveillance was the operational protocol (http://www.who.int/chp/steps/ Manual pdf). Disability status at 28 days was assessed by Modified Rankin Scale (mRS). mRS (0-2) denotes independent status and mRS (3-5) denotes dependant status and severe disability.

Results: At 28 days $29.8 \%$ died and $66.2 \%$ survived in Study I and $35.4 \%$ died and $56.5 \%$ survived in Study II. Nearly 38.5\% were left with moderate to severe disability at 28 days in Study I and $39.9 \%$ in Study II by Modified Rankin Scale (score 3-5). Major stroke parameters that remained unchanged between the two studies (I and II) were: mean age of stroke $(\mathrm{p}=0.9519)$, stroke subtype $(\mathrm{p}=0.151)$, risk factor profile $(\mathrm{p}=0.562)$, vital status at day $28(\mathrm{p}=0.168)$.

Conclusion(s): It is evident that burden of stroke is heavy for health care providers in developing country like India, particularly in the absence of organized stroke care facilities and domiciliary rehabilitation services. Role of public health nurse in co-ordinating needs of patients and caregivers for control of comorbid state with local physician is vital. WHO recommendations on prevention of stroke needs urgent implementation and judicious use of anti-platelets, anti-hypertensives and statins to prevent recurrent stroke will be helpful.

\section{0 \\ Pattern of Migraine in Upper Egypt; Clinico- Epidemiological Study}

Rageh T. ${ }^{a}$, Nagiub H. ${ }^{a}$, Farghaly W. ${ }^{a}$, Shehata G. ${ }^{a}$, Badry R. ${ }^{\text {, }}$ Badry N. ${ }^{\mathrm{b}}$, Kandil M. ${ }^{\mathrm{a}}$

${ }^{\mathrm{a}}$ Assiut University Hospital, Egypt; ${ }^{\mathrm{b}} \mathrm{Al}$ Azhar University Hospital, Egypt

Background: Migraine is a significant health problem due its frequency and accompanying morbidity. This study aims to estimate the prevalence of migraine headache among the population of Al-Quseir city and its impact on the patients' life.

Methods: This study forms a part of door-to door survey of major neurological disorders in Al-Quseir ciy, Red-sea governorate, Upper Egypt. All inhabitants ( $\mathrm{n}=33285$ persons) were screened through door to door (including every door) by three specialists of Neurology. Then, positive cases were subjected to clinical and neurological examination by three staff members of neurology each separately. Respondents were identified as suffering from migraine with aura, without aura and probable migraine as defined by the diagnostic criteria of the International headache society (IHS). Migraine Disability Assessment Scale (MIDAS) questionnaire was used to assess the impact of the disease on the patients' daily life.

Results: We identified 928 patients suffering from migraine with a female to male ratio 2.5: 1 . The life time prevalence was 2.8 / 100 with male prevalence of $1.6 / 100$ and female prevalence of 4.0 /100. The highest prevalence figures were found during early adult life (18-40 years) among both genders reaching a total prevalence of 4.77 / 100 (2.89 / 100 for male and 6.53 / 100 for female). Among migraine patients, most of the attacks recorded were of moderate to severe intensity (97\%). Although virtually $66 \%$ of migraine patients reported that headache significantly interfered with their daily activities, prophylaxis is only used by few patients (8.4\%).

Conclusion: Migraine headache represents a major health problem with adverse effects on patient's quality of life and deleterious impact on the patiens't functional and wellbeing.

\section{3 \\ Persons with Epilepsy Enrolled in the Ohio Medicaid Program: An Analysis of their Survival Patterns}

Koroukian S., Schiltz N., Bakaki P., Kaiboriboon K.

Case Western Reserve University, USA

Background: This section outlines importance, objectives/ aims or hypothesis of the study.

Because of the requirements associated with low income and disability, persons with epilepsy (PWE) on Medicaid are a particularly vulnerable to poor health outcomes. To identify possible areas of intervention aimed at reducing mortality in PWE, we examined survival patterns in PWE enrolled in the Ohio Medicaid program. 
Methods: In this section, design, subjects, and statistical analysis used in the study are briefly described.

We used linked 1991-2008 Ohio Medicaid claims and enrollment data and Ohio death certificate data. In addition to using comorbid conditions, we characterized PWE by whether their epilepsy was in remission (REM) for the last 36 months of follow-up (PWEs receiving anti-epileptic drugs, but without history of admission to the hospital or emergency department for seizures); refractory (REF) during these 3 years (those with 2 or more medications, and history of admission to the hospital or emergency department for seizures), or neither (ACTIVE). We conducted descriptive analysis, and developed a multivariable logistic regression model to study the association between each type of epilepsy with mortality, after controlling for demographics and comorbidities. We also conducted survival analysis, accounting for the aforementioned variables.

Results: In this section, main results of the study are presented as clearly as possible.

Our study population included 67, 038 PWEs age 18-64 years of age, and enrolled in Medicaid during the study period (mean age: 36.5 years). While $17.3 \%$ in PWE were identified as being in remission, $2.4 \%$ and $80.3 \%$ were characterized as having REF or ACTIVE epilepsy, respectively. The proportion of those who died was lowest in PWEs who had their disease in remission (9.3\%), and highest in those with refractory disease (35.0\%). Of those with ACTIVE disease, $20.3 \%$ died. Compared to those with ACTIVE disease, those in REM and REF epilepsy were 60\% less likely and 2.7 times more likely, respectively, to die during the study period (adjusted odds ratio (AOR): 0.41, (95\% Confidence Interval (CI): $0.38,0.43)$, and AOR: $2.65(2.37,2.97))$. The multivariable survival analysis yielded similar results.

Conclusion(s): In this section, importance of results of the study for practice and/or future research is briefly described.

Compared to those with ACTIVE or REF epilepsy, PWEs in seizure remission have favorable survival outcomes. The findings highlight the importance of seizure control in PWEs.

\section{8}

\section{Epidemiology of Parkinson's Disease in Cuiaba}

Vilela Gomes B. ${ }^{\mathrm{a}}$, Siqueira $H^{\mathrm{b}}{ }^{\mathrm{b}}$, Borges $E^{\mathrm{a}}{ }^{\mathrm{a}}$, Lellis B. ${ }^{\mathrm{b}}$, Torres $F^{\mathrm{b}}$, Mello S.R. ${ }^{\mathrm{b}}$, Rodrigues M. ${ }^{\mathrm{b}}$

aUniversity of Cuiaba, Brazil; ${ }^{b}$ Hospital Geral Universitario, Brazil

Background: Parkinson's Disease (PD) is a neurodegenerative disease, idiopathic and irreversible, affect accounted for approximately $2 \%$ of the population over age 65 and $0.3 \%$ of the general population. Therefore, screening of PD in individuals members of the Centre for the Elderly Padre Firmo and Maria Ignes becomes fundamental to the epidemiological data survey of this disease in Cuiabá, and to compare these with those presented today by literature. OBJECTIVE: Identify individuals with PD in Centers the Elderly.

(this section outlinesimportance, objectives/aims or hypothesis of the study).
Methods: This is a population study in two phases with the first stage screening for movement disorders. All individuals positive for the disorder of movement were assessed using a structured questionnaire containing demographic data, clinical features and diagnosis of PD according to the London Brain Bank: tremor, bradykinesia, rigidity and / or postural instability.

(in this section, design, subjects, and statistical analysis used in the study are briefly described).

Results: Cuiabá has the general population of 551, 098 inhabitants and $8.1 \%$ of individuals over 60 years, among these 258 people were assessed, representing $0.57 \%$ of this group. The average age of the sample was 68.7 years wich $80.6 \%$ female and $19.4 \%$ male. We identified 24 patrons with idiopathic PD, 2 PD of vascular origin and six drug-induced $\mathrm{PD}$, the main drug-related cinnarizine and flunarizine. The prevalence of IPD in this population was $9.3 \%$. The analysis of data on quality of life in the sample presents PDQ-39 with a mean of 19.4 (7.89 to 35.89), Hohen and Yahr stage 1 and 2 with $36 \%$ to $64 \%$. We did not identify any individual in the sample with less than $80 \%$ ADL.

(in this section, main results of the study are presented as clearly as possible).

Conclusion(s): The present data show that the prevalence of $\mathrm{PD}$ in these patients was higher than that reported in the literature today and a better quality of life in newly diagnosed individuals, indicating the importance of this morbidity in the elderly population, requiring active research in this group for early diagnosis and early treatment.

(in this section, importance of results of the study for practice and/or future research is briefly described).

\section{0 \\ Multicenter External Validation of the ASTRAL Score Predicting Functional Outcome After Acute Ischemic Stroke: Result from China National Stroke Registry}

\author{
Wang Y. ${ }^{\mathrm{a}}$, Zheng H. ${ }^{\mathrm{a}}$, Gaifen L. ${ }^{\mathrm{a}}$, Wang Y. ${ }^{\mathrm{a}}$, Fang J. ${ }^{\mathrm{b}}$, Michel P. ${ }^{\mathrm{c}}$, \\ Papavasileiou V. ', Ntaios G. , Liu L. a , Dong K. a, Wang C. a , Zhao X. , \\ Wang D.Z.

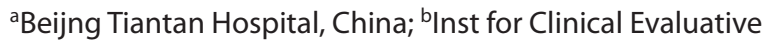 \\ Sciences, Toronto, Canada; 'Centre Hospitalier Universitaire \\ Vaudois and University of Lausanne, Switzerland; dIllinois \\ Neurological Institute Stroke Network, OSF Healthcare \\ Systems, College of Medicine at Peoria, USA
}

Background: The ASTRAL score was developed as a prognostic tool for acute ischemic stroke. This scoring system has been reported to be able to predict the short-term outcome accurately in both the derivation and validation cohorts originated from Europe. The purpose of this study was to validate the ASTRAL score in the Chinese stroke population and explore its predictive value for long-term outcome.

Methods: We applied the ASTRAL score to patients presenting with an acute ischemic stroke registered in the China National Stroke Registry that included 132 study sites in Mainland China from 2007 to 2008 . The clinical outcome was assessed by the modified Rankin Scale (mRS) at 3, 6 and 12 months after onset. 
Unfavorable outcome was defined as modified Rankin scale $>2$. Areas under the curve (AUC) of the corresponding receiver operator characteristic were calculated to quantify the prognostic value.

Results: Of the 3755 patients who met the study criteria, $1473(39.66 \%)$ had a unfavorable outcome. Compared to those with good outcome, the patients with unfavorable outcome were older(odds ratio $[\mathrm{OR}]=1.22 ; 95 \% \mathrm{CI}, 1.18$ to 1.26 ), had significantly higher NIHSS scores on admission $(\mathrm{OR}=1.20 ; 95 \% \mathrm{CI}, 1.18$ to 1.22$)$, delay from stroke onset to admission $(\mathrm{OR}=1.27 ; 95 \% \mathrm{CI}$, 1.07 to 1.49$)$, abnormal glucose levels $(\mathrm{OR}=1.33$; $95 \% \mathrm{CI}, 1.11$ to 1.59), severe impaired level of consciousness $(\mathrm{OR}=1.35 ; 95 \% \mathrm{CI}$, 1.03 to 1.76$)$ and visual field $\operatorname{defect}(\mathrm{OR}=1.01 ; 95 \% \mathrm{CI}, 0.73$ to 1.41). The area under the ROC curve for the ASTRAL score predicting unfavorable outcome was $0.821,0.815$ and 0.817 for 3,6 and 12 months on follow up.

Conclusion(s): This study externally validated the ASTRAL score in Chinese stroke population. ASTRAL score has shown to be a useful tool that can be readily applied in clinical practice.

\section{2 \\ Short-Term Trends in Stroke Profile and Outcome in Mumbai Stroke Registry}

Dalal P., Bhattacharjee M., Vairale J.

LKMM Trust Research Centre, India

Background: In developing countries where health statistics are incomplete, stroke surveillance on representative population samples may be justified. Such resurveys should be on well defined stroke protocol at reasonable interval.

Methods: We carried out trend studies in 2005-06 and in 2009 using identical and well defined protocol (http://www.who.int/ chp/steps/Manual pdf) in the same census verifiable district (H-ward), by the same team. During the period intensive campaign on stroke awareness continued but there was no intervention.

Results: It was observed that the stroke profile parameters on "first-ever-strokes" in 2005-06 was almost replicated in 2009. The indices which remained unchanged in this period were: mean age of stroke $(\mathrm{p}=0.9519)$, stroke subtype $(\mathrm{p}=0.151)$, risk factor profile $(p=0.562)$, vital status at day $28(p=0.168)$. The recovery at 28 days both by MRS $(p=0.1938)$ and Barthel Index $(p=0.1336)$ did not show any statistical significance in 2005-06 and in 2009. Hospital admissions in both the studies was unchanged (60-67\%), whereas nursing home admissions were more in 2009 (25.1\%) compared to 2005-06 (15.3\%) which was statistically significant(p $=0.0364$; OR: 1.8133$)$.

Conclusion(s): All patient characteristics like mean age, gender, vital status at day 28, Modified rankin scale at day 28, stroke sub type, and risk factor profile were nearly identical, indicating that such quick re-surveys may not be truly indicative of trend. This re-survey suggests that a more cost-efficient approach to stroke re-surveillance is to study the same population once in 5 or 10 years rather than earlier, with a view to design awareness and intervention strategies. This may help.

\section{Session 04: Treatment/Management}

\section{3 \\ Treatment of Epilepsy in Daily Clinical Practice: Have Outcomes Improved Over the Past 10 Years?}

Wassenaar M. ${ }^{\mathrm{a}}$, Leijten F. ${ }^{\mathrm{a}}$, Van Der Linden P. ${ }^{\mathrm{b}}$, Uijl S. ${ }^{\mathrm{a}}$, Egberts T. ${ }^{\mathrm{c}}$, Carpay $H^{\text {b }}$

aUMC Utrecht, The Netherlands; ${ }^{\text {b}}$ Tergooi hospitals, The Netherlands; 'Utrecht University, The Netherlands

Background: New anti-epileptic drugs (AEDs) have increased epilepsy treatment options and have been promoted for better safety profiles. It is unknown if this has resulted in better patient outcomes in daily practice. We describe changes over a 10 -year time period in health-related quality of life (QoL), seizure control and adverse effects (AEs) in a community-based sample of epilepsy patients and assess potential predictors of QoL.

Methods: We compared two cross-sectional communitybased studies in the same Dutch suburban region performed in 2000 and 2010. These addressed treatment outcomes in epilepsy. Epilepsy patients were identified using pharmacy records. We included 344 and 247 patients respectively. Outcomes: QoL, seizure control and AEs were measured by dedicated questionnaires such as QOLIE-31. Factors predicting QoL (AEs, seizure control, seizure acceptability, demographic, epilepsy- or treatment related determinants) were assessed by multivariable linear regression.

Results: New AED use was $9 \%$ in 2000 and $34 \%$ in 2010 . We found no differences in QOLIE-31 scores (72.5 versus 72.4) or in complete seizure control (51\% versus 55\%). AEs were reported more often (59\% versus $82 \%$ ). Seizure control and the occurrence of AEs were the main independent predictors of QoL in both studies explaining $70-75 \%$ of the variance.

Conclusion: We found no evidence that the availability of new AEDs has improved QoL in epilepsy patients over the past decade. Seizure control was unchanged and AEs were reported more frequently. The low proportion of complete seizure control and high rate of AEs in this community-based sample suggests that treatment is still suboptimal. Due to the important contribution of AEs to QoL, more efforts to reduce AEs are likely to benefit epilepsy patients. 


\section{0}

TYSEDMUS: Observational Prospective Follow-Up Study of Patients with MS and Treated with Natalizumab in France Using the French EDMUS (European Database for Multiple Sclerosis) Network Five-Year Results

\section{Vukusic S. ${ }^{\mathrm{a}}$, Passante N. ${ }^{\mathrm{b}}$, Dufour M. ${ }^{\mathrm{a}}$, Rocher F. ${ }^{\mathrm{b}}$, Van Ganse E. ${ }^{\mathrm{c}}$,} Clanet M. ${ }^{d}$, Falip E. ${ }^{\mathrm{e}}$, Confavreux $\mathrm{C}^{\mathrm{a}}$

aHôpital Neurologique et Fondation EDMUS pour la Sclérose

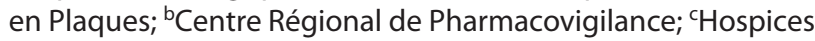
Civils de Lyon; ${ }^{d}$ Hôpital Purpan; ${ }^{\mathrm{e}}$ Agence Nationale pour la Sécurité des Médicaments, France

Background: In November 2007, a national institution-driven Risk Management Programme (RMP) has been set up in France by the French Medicines Agency (ANSM), to minimize the risks and monitor the safety and efficacy of Natalizumab (NZ) in daily practice. It is focused on TYSEDMUS, a pharmaco-epidemiological study based on the French network of neurologists using EDMUS. The main objective is to establish the safety profile of NZ in real life settings. Secondary objectives are to describe the efficacy in terms of relapses and disability and the patterns of use.

Methods: TYSEDMUS is a multicentre observational cohort study including all patients exposed at least once to NZ. Patients' characteristics, utilisation patterns and occurrence of adverse events are described. The study duration is planned to be at least 5 years.

Results: In May 2012, circa 5000 MS patients were treated with NZ in France, with 3918 referred to the OFSEP Coordinating Centre. The median follow-up was 23.6 months. $5 \%$ experienced a serious adverse event: 21 Progressive Multifocal Leukoencephalopathy (10 reported in TYSEDMUS), 1 fatal acute leucoencephalopathy due to Mycoplasma pneumonia, 1 VZV retinitis, 1 herpetic encephalitis and 18 severe allergic reactions. The immediate infusion-related tolerance was good. $18 \%$ of the patients definitively discontinued treatment. The annualised relapse rate decrease by $83 \%$ after 1 year and $85 \%$ after 2 years. The mean EDSS score decreased from $3.7 \pm 1.8$ to $3.3 \pm 1.9$ after 1 year. The French neurologists usually followed the RMP recommendations.

Conclusion(s): Results are confirmative regarding safety data, but also efficacy and good practice of use of NZ by French neurologists. Updated results will be presented at the meeting.

\section{5}

The MRI DRAGON Score: 3-Month Outcome Prediction After IV Thrombolysis for Ischemic Stroke

Turc G. ${ }^{a}$, Apoil M. ${ }^{\text {, }}$ Tataru A.M. ${ }^{a}$, Allibert R. ${ }^{a}$, Meder J.-F. ${ }^{a}$, Mas J.-L. ${ }^{\text {, }}$ Baron J.C. ${ }^{\text {a }}$ Oppenheim C. ${ }^{\text {, }}$ Touze E. ${ }^{\text {a }}$

aUniversité Paris Descartes Sorbonne Paris Cité, Centre de psychiatrie et de neurosciences, INSERM UMR S894, Hôpital Sainte Anne, Paris, France; ${ }^{b}$ Centre Hospitalier Sainte-Anne, France

Background: The DRAGON score, which includes clinical and CT scan-based parameters, showed a high specificity to predict 3-month outcomes in acute ischemic stroke (AIS) patients treated by IV-tPA. We adaptated this score for the increasing number of patients undergoing MR as the first-line imaging.

Methods: We reviewed all anterior circulation AIS patients treated within $4.5 \mathrm{~h}$ by IV-tPA between 2003 and 2012 in our academic center, where MRI is systematically implemented as firstline diagnostic work-up. We derived the MRI DRAGON score keeping all clinical parameters of CT-DRAGON (age, initial NIHSS and glucose level, prestroke handicap, onset to treatment time [OTT]), and considering proximal middle cerebral artery (MCA) occlusion on MRI and DWI ASPECTS score $\leq 5$ as new imaging parameters. Unfavorable 3-month outcome was defined as modified Rankin scale $>2$. We calculated C-statistics as a measure of predictive ability, and performed an internal cross-validation.

Results: 228 patients (Mean \pm SD age 67.3 \pm 14.9 , median(IQR) baseline NIHSS score 14(8-19), 145(64\%) with proximal MCA occlusion, 45(20\%) with $\leq 5$ DWI ASPECTS score) were included. Unfavorable outcome was observed in 98(43\%) patients and was significantly associated with all parameters of the MRI DRAGON score in multivariate analysis, except for OTT (non-significant trend). The c-statistic was 0.84 (95\%CI 0.78-0.89) for unfavorable outcome prediction. All patients with a MRI DRAGON score $\leq 2$ $(\mathrm{n}=20)$ had a favourable outcome, while all patients with $\mathrm{a} \geq 8$ score $(\mathrm{n}=11)$ had an unfavorable outcome.

Conclusion(s): The MRI DRAGON score is a new simple tool to predict 3-month outcome in patients treated by IV t-PA for AIS and imaged by MR, and may help optimizing acute management.

180

Non-Inferiority of Azathioprine versus Interferon Beta for Relapsing Remitting Multiple Sclerosis: A Multicenter Randomized Trial

\begin{abstract}
Benedetti M.D. ', Massacesi L. ', Tramacere I. ', Filippini G. ', La Mantia L. ', Solari A. ', Milanese C. ${ }^{c}$

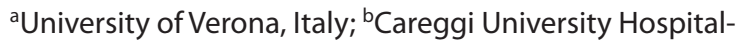
University of Florence, Italy; 'Istituto Neurologico Carlo Besta Milano, Italy
\end{abstract}

Background: Since the approval of interferon beta (IFNs) as a first-line treatment of relapsing-remitting multiple sclerosis (RRMS), azathioprine (AZA) has mainly been used as a second 
line therapy, although a direct comparison between the two treatments has never been performed. We present the results of a multicenter randomized controlled trial, comparing the efficacy and safety of AZA on clinical and MRI outcome measures with the currently available IFNs.

Methods: Inclusion criteria were: relapsing-remitting course, at least 2 relapses in the last 2 years, age 18-55, Expanded Disability Status Scale (EDSS) 1.0-5.5. Eligible patients recruited in 30 Italian MS Centers were randomly assigned to AZA or to an approved IFN and followed for 2 years. Primary outcome was efficacy on relapse rate. Key secondary outcome was the number of MRI new brain lesions in T2 weighted scans. Other clinical, safety, neuroradiologic and health related quality of life end-points were also analyzed. The study was single-masked: at each participating center the patients, aware of the treatment received, were assessed by a treating (unblinded) and by an examining (blinded) neurologist. Brain MRI images were centrally analyzed by two independent experts, unaware of treatment and patients outcome. The efficacy of the two treatments was compared by applying a noninferiority design.

Results: One-hundred-fifty patients were randomized in two groups (77 AZA and 73 IFN) that resulted highly comparable as for clinical and demographic baseline characteristics. Clinical evaluation was completed in 127 patients (62 AZA, 67 IFN). Annualized relapse rate in the AZA group was lower $(0.26,95 \%$ C.I. $0.19-0.37)$ than in the IFN group (0.39, 95\% C.I. $0.30-0.51)$. Non-inferiority analysis showed that AZA was at least as effective as IFNs on this outcome measure (relapse rate ratio AZA/IFN = 0.67 , one sided $95 \% \mathrm{CI}=0.96 ; \mathrm{p}<0.01$ ). The MRI outcomes were analyzed in 97 patients (50 AZA and 47 IFN). Annualized new T2 lesion rate was $0.76(95 \%$ CI $0.61-0.95)$ in the AZA group and 0.69 (95\% CI $0.54-0.88)$ in the IFN group and non-inferiority analysis showed that the effect of AZA was at least $73 \%$ of the IFNs (rate ratio AZA/IFN $=1.10$, one sided 95\% CI $=1.45, \mathrm{p}<$ 0.01 ). The number of patients with an adverse event was similar in the two groups, although the total number of adverse events was higher under AZA when lymphocytopenia was included. The number of treatment discontinuations due to adverse events was also higher in the AZA group. These events were more frequent in the first months of treatment with AZA, whereas in the IFN group discontinuations occurred mainly during the second year.

Conclusion(s): Our data indicate that in RRMS patients AZA is at least as effective as IFNs. Given the convenience of the oral administration and the low cost for the Heath Service providers, AZA should be used as a first line treatment in RRMS.

\section{9 \\ Impact of Anti-Epileptic Drug Treatment on the Natural History of Patients with Cerebral Palsy Exhibiting Interictal Epileptiform Discharges Even Without Clinical Epilepsy}

Jaseja Harinder

G R Medical College, India

Cerebral palsy (CP) is tradionally known to be a non-progressive motor disorder. This belief inculcates a feeling of complacence and at times even helplessness in the minds of clinicians and caretakers of patients with CP resulting in a significantly constrained approach to the management of such patients. The disorder is associated with a high incidence of epilepsy, interictal epileptiform discharges (IEDs) and cognitive impairment. Co-morbidity with epilepsy is estimated to be as high as $30-50 \%$, often with the epileptic attacks exhibiting refractoriness to the conventional anti-epileptic drugs (AEDs); further, the attacks are frequently subtle or micro-seizures that are often missed and unreported by the patients and/or caretakers. In view of the prevailing impression that the natural course of $\mathrm{CP}$ disorder is generally unalterable, the AED therapy is limited to $\mathrm{CP}$ patients presenting with unambiguous epileptic attacks leaving a significant section of $\mathrm{CP}$ patients with micro seizures and/or IEDs untreated. In the context of growing evidence in favor of causaleffect relation between IEDs and cognitive impairment, this paper is intended to propose a new guideline to include a mandatory EEG in the management protocol of all CP patients and treatment of IEDs (if detected) irrespective of seizure occurrence. IEDs have been shown to cause premature $\mathrm{Ca}++$ induced death of cortical neurons that may result in aggravation of existing cognitive impairment and cortical dysfunctioning. Uncontrolled reports and a few preliminary randomised controlled trials of suppression of IEDs by AEDs have yielded significant improvement in psychosocial function. Further, recent studies have reported improvement in cognitive impairment and even onset of speech upon treatment of IEDs detected in mentally challenged patients' EEG even without a history of clinical epilepsy. Yet another recent study has also suggested that IEDs in CP patients without seizure history predisposes them to subsequent unprovoked seizures or epilepsy. Thus, it is claimed that the inclusion of a mandatory EEG in the management protocol of all patients with CP and treatment of IEDs (if detected) can definitely alter its natural history and course and yield a favorable impact on the prognosis of patients with $\mathrm{CP}$ with IEDs and/or subtle or micro seizures. 
257

\section{The Influence of Immunomodulatory Therapy on Vaccination Response Among Patients with Multiple Sclerosis}

Myhr K.M. ${ }^{\mathrm{a}}$, Olberg H.K. ${ }^{\mathrm{a}}$, Cox R.R. ${ }^{\mathrm{b}}$, Nastbakken J.K. ${ }^{\mathrm{b}}$, Aarseth J.H. ${ }^{\mathrm{a}}$, Vedeler C.A. ${ }^{\text {a }}$

${ }^{a}$ Haukeland University Hospital; b University of Bergen, Norway

Background: To explore whether immunomodulatory treatment influenced the immune response to pandemic H1N1 vaccination in 2009 (monovalent AS03 adjuvanted vaccine) among patients with multiple sclerosis (MS).

Methods: We investigated the immune response to $\mathrm{H} 1 \mathrm{~N} 1 \mathrm{vac}-$ cination among 131 MS patients and 216 health care workers (controls) during the pandemic of 2009. We recorded demographic variables (age, sex, disease onset), clinical variables and immunomodulatory treatment (interferon beta, glatiramer acetate, natalizumab and mitoxantron).

The vaccine response was evaluated by the haemagglutination inhibition (HI) assay, commonly used to measure influenza specific antibody responses after vaccination. A HI titre $\geq 40$ is established as a surrogate correlate of protection.

Results: Only $27.4 \%$ of MS patients that received immunomodulatory treatment had protection from the vaccine after 10 months, compared to $43.5 \%$ of the controls after 12 months. The specific protection levels divided according to medication were: interferon beta (44.4\%), glatiramer acetate $(21.6 \%)$, natalizumab (23.5\%) and mitoxantron (0.0\%).

Younger patients had significantly better protection than older patients. Self-reported influenza illness was associated with significantly higher titres. Gender, family members undergoing influenza like illness, or Tamiflu intake did not influence the immune response.

Conclusion(s): MS patients that receive glatiramer acetate, natalizumab and mitoxantron had reduced long-term protection after $\mathrm{H} 1 \mathrm{~N} 1$ vaccination. Interferon beta treated patients had a normal vaccine response. These preliminary findings suggest that MS patients on other immunomodulatory therapy than interferon beta may need two doses of vaccines in a new pandemic of influenza.

\section{Session 05: Neurosurgery}

\section{4 \\ Cryosurgical Method at Open Operative Treatment in the Patients with Brain Tumors}

Volov M., Mozhayev S.

N.P. Bechtereva Institute of Human Brain of Russian Academy of Science, Russian Federation

Background: Purpose: to define opportunities of cryosurgical action in an improvement of results of brain tumors surgical treatment.

Methods: 129 patients with brain tumor, with indications for open operative intervention, were examined: the I group - 62 patients which were operated with application cryosurgical method (26 men, 36 woman, mean age 44, 06 2 , 1 years) and the II group - 67 patients which were operated without of cryosurgery method (32 men, 35 woman, mean age 46, 25 $\pm 1,71$ years). Cryosurgical action during operative intervention used in the form of: cryofixation, cryoresection, cryodestruction, cryoextirpation, cryocoagulation, and combination of the techniques. Computer, magnetic-resonance and positron emission tomographie of a brain were performed on the all patients before, after operation and in follow-up period (53, 57 $\pm 4,54$ month).

Results: Total brain tumors removal was reached in more cases in the I group in comparison to control group (37 and 28 patients accordingly, $\mathrm{p}=0,04)$. Duration of operation with application of cryosurgical method did not vary between the groups significantly $(263,93 \pm 13,07 \mathrm{~min}$ and $257,53 \pm 19,5 \mathrm{~min}$ - in control; $\mathrm{p}=0,71)$. In follow-up period a number of the patients with continued growth of a tumor was less in the I to group (I group $30,65 \%$ and II group - 56, $72 \% ; p=0,01)$. Death from continued growth of a tumor occured more rare in the I group in comparison with the II group of the patients, and the difference was veliable $(\mathrm{p}=0,02)$.

Conclusion(s): An application of cryosurgical action during the open operative intervention in patients with a brain tumor increases a rate of tumor removal radicalism and decreases a rate of continued growth of a tumor. 


\section{Session 07: Neuroimaging Studies}

\section{9 \\ Association Between Cerebral Microbleeds, Hippocampal Volume and Cognitive Function in Old Age}

Maier I. ${ }^{\text {, }}$, Van der Grond J. ${ }^{\text {a, }}$ Konishi J. ${ }^{\mathrm{b}}$, van Es A. ${ }^{\mathrm{a}}$, Bollen E. a, Middelkoop H. ${ }^{a}$, Blauw G.J. ${ }^{a}$, Westendorp R. ${ }^{\text {, }}$, De Craen A. , Van Buchem M. ${ }^{a}$

aLeiden University Medical Center, The Netherlands; ${ }^{\text {KKobe }}$ University, Japan

Background: Alzheimer's disease (AD) and cerebral microbleeds (CMBs) are highly prevalent in the elderly. CMBs with a lobar distribution are suggestive of cerebral amyloid angiopathy (CAA). Both $\mathrm{AD}$ and CAA are characterized by beta-amyloid $(\mathrm{A} \beta)$ accumulation. Since a common underlying pathophysiological mechanism for $\mathrm{AD}$ and $\mathrm{CAA}$ has been hypothesized, we assessed hippocampal volume and cognitive function in subjects with and without CMBs.

Patients and Methods: All data come from the MRI-substudy of PROSPER (PROspective Study of Pravastatin in the Elderly at Risk). For the present analysis we included 430 subjects with an average age of 75 years. Presence of CMBs, hippocampal volume, and cognitive function were assessed at baseline and after 33 months.

Results: A total of 105 subject (24\%) had CMBs, from which 47 (45\%) had CMBs with a lobar distribution. There was no difference in hippocampal volume or cognitive test scores between subjects with and without cerebral microbleeds. Moreover, subjects with cerebral microbleeds with a lobar distribution did also not have a lower hippocampal volume or lower cognitive test performance. There were also no significant changes over time in hippocampal volume or cognitive test scores between study groups.

Conclusion: Neither the presence nor a lobar distribution of CMBs is associated with hippocampal volume and cognitive function. This finding does not support the hypothesis of a common underlying pathophysiological mechanism for CAA and AD.
278

\section{Diagnostic Challenges of Primary Thalamic Gliomas Identification of a Minimally Enhancing Neuroradiological Subtype with Aggressive Neuropathology and Poor Clinical Outcome}

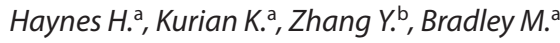

${ }^{a}$ Frenchay Hospital UK, United Kingdom; b'Bath Hospital, United Kingdom

Background: To evaluate neuropathology and neuroradiology in the diagnosis and clinical outcome of a retrospective cohort of thalamic gliomas.

Methods: Neuropathological and neuroradiological review was undertaken of 25 cases of radiologically suspected thalamic glioma (excluding childhood pilocytic astrocytoma) over an 8 year period (2004-2012) at Frenchay Hospital and compared to the clinical outcome.

Results: In $48 \%(12 / 25)$ there was a difference in neuropathological and suspected neuroradiological grading of the lesion of one or more grades. In $5 / 12(42 \%)$ cases the neuroradiology was lower grade than the pathology. In $4 / 5(90 \%)$ of these cases, we identified a minimally enhancing subtype where the neuroradiology was predicted to be lower grade than neuropathology. In $4 / 12$, (33\%) the suspected neuroradiology grade was higher than the final pathology. In $3 / 4,(75 \%)$ of these cases the suspected neuroradiology grade was higher than the neuropathology possibly due to unusual differentiation within the thalamic glioma (central neurocytoma, anaplastic oligodendroglioma, and diffuse astrocytoma with piloid features). In $25 \%$ (3/12) the biopsy was nondiagnostic.

Neuropathology was a better predictor of clinical outcome than neuroradiology. 9/10 (90\%) WHO 4 gliomas and 8/9 (88\%) Grade 3 gliomas on neuropathology were dead between $3-7$ years after diagnosis. 3/3 (100\%) Grade 2 gliomas on neuropathology were alive 3-7 years after diagnosis. 2/3 (67\%) of the non-diagnostic cases were alive 3-7 years after biopsy. In 1/3 (33\%) of the nondiagnostic cases the outcome was unknown.

Conclusion(s): Diagnosis of primary thalamic glioma is challenging. This is the first description of a pattern of diffuse infiltration with minimal enhancement on imaging within the thalamus that represents high grade glioma. Neuropathology is overall the best predictor of clinical outcome. Knowledge of the value of neuropathology in predicting clinical outcome may inform a decision to biopsy suspected thalamic tumours. 


\section{Session 08: Neurogenetics}

\section{3 \\ Epidemiological and Genetic Studies of Parkinson's Disease in Russia}

Pchelina S., Emelyanov A., Schwarzman A., Yakimovskii A.

Pavlov's State Medical University, Russian Federation

Background: Parkinson's disease (PD) is a common neurodegenerative disorder. Although PD is generally a sporadic neurological disorder mutations at least in five genes (SNCA, PARK2, PINK1, DJ1 and LRRK2) have been shown to course a Mendelian pattern of PD inheritance. Mutations in the GBA gene are high risk factors for PD development.

Methods: We conducted a cross-sectional analysis of 486 PD patients in Russia to estimate the epidemiological characteristics of tremor-dominant PD and early onset PD with age at onset before 50 years (EO-PD). The prevalence of tremor-dominant form and EO-PD have been documented via clinical examinations and questionnaire surveys in two groups of randomly selected PD patients referred to clinics of Pavlov State Medical University: 230 were recruited from 1999-2001 and another 256 - from 2008-2010. To investigate the epidemiology of inherited forms of PD in Russia we screened mutations in the LRRK2, PARK2, SNCA and GBA genes in 100 patients with familial PD.

Results: The frequency of EO-PD did not differ between the groups and was $19.2 \%$ and $16.4 \%$, respectively. The increased frequency of tremor-dominant $\mathrm{PD}$ was found in patients recruited in $2008-2010$ (39.0\% compared to $24.6 \%, \mathrm{p}<0.001)$. Among patients with familial PD 8 revealed mutations in the LRRK2 gene (7 G2019S, 1 - V1613A), 1 patient had duplication of the SNCA gene, 1 patient had heterozygous deletion of exon 3 in the PARK2 gene and 3 patients had the L444P mutation in the GBA gene.

Conclusion(s): Our results suggest the increase of tremordominant PD over past ten years and showed that the G2019S LRRK2 mutation is the main cause of familial PD in Russia.

97

Relevance of Endothelial Nitric Oxide Sintase, Elastin and Endoglin Genetic Variants in Familial Intracranial Aneurysm

Gregorio M.L., Pinhel M., Florim Greiciane M., Amorim G.S., Nakazone M.A., Martins D.P., Silva S., Crestani A.M., Santos M.L.T., Ferraz-Filho J.R.L., Rossi Silva S.D., Tognola Waldir A.

Sao Jose do Rio Preto Medical School, Brazil

Background: Intracranial aneurysm (IA) risk factors can be environmental like smoking and alcoholism, and genetic like variants of endothelial nitric oxide sintase (eNOS), elastin (ELN) and endoglin (ENG). Analyze eNOS, ELN and ENG polymorphisms, environmental risk factors and the association with IA.
Methods: 836 individuals divided in 6 groups: G1- 40 (familial IA); G2- 176 (G1 family); G3 - 113 (sporadic IA); G4 - 277 (G3 family); G5 - 104 (controls); G6 - 126 (G5 family). Polymorphisms analysis eNOS, ELN and ENG was done by PCR (polymerase chain reaction). Significance level $\mathrm{P}<0.05$.

Results: eNOS: allele A more prevalent in G1 (0, 93), G2 (0, $83), \mathrm{G} 3(0,79), \mathrm{G} 4(0,89)$ than $\mathrm{G} 5(0,61)$ and $\mathrm{G} 6(0,75 ; \mathrm{P}<0$, 0001). A/A genotype more frequent in G1 (86\%); G2 (77\%) G3 $(79 \%)$ and G4 (78\%), than G5 (26\%) and G6 (50\%; P < 0, 0001). ELN: similarity between groups $(\mathrm{P}>0,05)$. ENG: allele Wt more prevalent in $\mathrm{G} 5(0,81)$ than in $\mathrm{G} 1(0,61 ; \mathrm{P}=0,01)$; allele I more prevalent in $\mathrm{G} 2(0,30)$ than $\mathrm{G} 6(0,19 ; \mathrm{P}=0,003)$ and in $\mathrm{G} 3(0,34)$ than $\mathrm{G} 5(0,24 ; \mathrm{P}=0,027)$. Genotype -/Wt more frequent in G5 (89\%) than G1 (69\%; P = 0, 009) and in G2 (77\%) than G4 (88\%; P $=0,003)$. Genotype II prevailed in G2 $(22 \%)$, rather than G6 $(8 \%$ $\mathrm{P}=0,001)$. Smokers and alcoholics more prevalent in $\mathrm{G} 1(79 \%$; $40 \%$, respectively) and G3 (61\%; 36\%), than G5 (29\%; 20\%; P < , 05).

Conclusion: eNOS and ENG genetic polymorphisms are associated with IA, differentiating familial or sporadic IA and controls, as well as smoking and alcoholism.

\section{5 \\ Genetic Epidemiology of Tay-Sachs Disease: The Prevention of a Rare Disease \\ Salviati A. ${ }^{a}$, Pisoni N. ${ }^{a}$, Taioli F. ${ }^{a}$, Zidanes Lira A. ${ }^{a}$, Alberti D. ${ }^{\text {, }}$ Belli S. ${ }^{b}$, Bertorelli R. ${ }^{c}$ \\ aUniversity of Verona, Italy; ${ }^{\text {b} A P S S ~ T r e n t o, ~ I t a l y ; ~ ' U n i v e r s i t y ~ o f ~}$ Trento, Italy}

Background: Tay-Sachs disease is a fatal autosomal recessive disorder caused by hexosaminidase A deficiency. Its frequency is high in Ashkenazi Jews ( 1 in 30) and in some isolated populations. The aim of this study was heterozygote screening for reproductive counseling in a small Italian village in which an unusual frequency of Tay-Sachs disease had been detected. This section outlines importance, objectives/aims or hypothesis of the study.

Methods: The population of S. Lorenzo in Banale (TN), Northern Italy, at June 30th 2010 was 1177 (587 women and 590 men). Three Tay-Sachs cases have been diagnosed in the last 50 years. An educational program supported by the local administrative, health and ecclesiastic authorities was launched in the community to determine the frequency of Tay-Sachs carriers. Hexosaminidase A was dosed in the blood of consenting subjects and the screening was completed by molecular diagnosis on genomic DNA (the mutation detected was c.1183delG in exon 11 of gene HEXA). Christening Parish registers were reviewed since 1545 to 1923.

Results: Out of 587 women $116(19,7 \%)$ participated in the screening. Fifteen carriers were detected, corresponding to a carrier frequency of 1 in every 39 . The minimal prevalence of carriers in this village is 2.5 per 100 women, considering as non-carriers all the women who did not participate. This is the third population for Tay-Sachs frequency, the second in Europe after Ashkenazi Jews. Genetic counseling was provided both before and after 
screening. Confidentiality of test results and privacy were fully respected. From parish registers, to all intents considered birth certificate registers, a birth rate fall was observed in 1630, the year of epidemic Black Death in Europe, in the neighbouring villages but not in S. Lorenzo in Banale.

We detected an isolated population with a very high frequency of Tay-Sachs disease carriers, probably result of a founder effect. To explain the high frequency of carriers a heterozygote advantage is suspected.

\section{8 \\ IDH Mutation Analysis in Gliomas as a Diagnostic and Prognostic Biomarker}

Haynes H. ${ }^{a}$, Kurian K. ${ }^{a}$, Crosby C. ${ }^{b}$, Hopkins K. ${ }^{c}$, Williams M. ${ }^{b}$

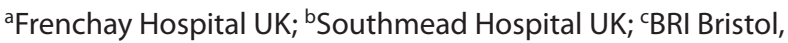
United Kingdom

Introduction: There is a high rate of IDH1/2 mutations in low grade gliomas and in high grade gliomas deriving from them. IDH analysis of gliomas is a novel method of classification and an independent prognostic marker. We compared antibody and sequencing methods for the detection of IDH mutations.

Method: 88 samples from 74 patients were identified: 16 WHO Grade 2 gliomas, 30 WHO Grade 3 gliomas and 28 WHO Grade 4 GBM. 31 samples were excluded from molecular genetic analysis due to insufficient sample available for DNA extraction. For immunohistochemistry: sections were stained with antiIDH1R132H antibody. For sequencing: DNA was extracted from fresh, frozen tissue. Sanger sequencing of the R132 region of IDH1 and the R172 region of IDH2 was carried out.

Results: $20 / 72$ cases (28\%) were positive for the R132H IDH1 mutation by antibody. An IDH1 mutation was detected by molecular genetics in $21 / 57$ cases (37\%) and no IDH2 mutations were detected (6\% expected from previous studies). 5/21 cases (24\%) had rare IDH1 mutations not detected by immunohistochemistry (7\% expected). Three of these cases displayed the p.Arg132Cys mutation (x2 anaplastic astrocytomas; x1 fibrillary astrocytoma) and two displayed p.Arg132Gly (x1 anaplastic astrocytoma; $\mathrm{x} 1$ anaplastic oligoastrocytoma). Where sufficient tissue was available, immunohistochemistry and DNA analysis were fully concordant for the p.Arg132His mutation.

We found a high rate of IDH1 mutations in lower grade lesions (WHO grade II \& III) (54\%) and a low rate in GBMs (7\%). Both grade II gliomas and anaplastic astrocytomas showed a statistically different distribution of IDH1 mutation load compared to GBMs ( $\mathrm{p}<0.0001 ; \mathrm{p}=0.0021$ respectively).

Conclusion: A rationalised combined approach involving $\mathrm{R} 132 \mathrm{H}$ antibody testing and sequencing of negative cases would be ideal for the detection of IDH1 mutations - antibody testing is cheaper than sequencing but sequencing demonstrates rare IDH1 mutations not detected by immunohistochemistry. IDH1 mutation analysis may also have a novel diagnostic role in tumour samples (or biopsies) lacking classical histological features, for example differentiating lower grade tumours from GBM.

\section{0}

Survey of Hereditary Ataxias and Spastic Paraplegias in Portugal: A Population-Based Prevalence Study

\author{
Ruano L. a, Coutinho P. ${ }^{\mathrm{b}}$, Loureiro J L. a, , Cruz Vitor T. a , Barros J.', \\ Tuna A. ${ }^{\mathrm{c}}$, Barbot C. ${ }^{\mathrm{d}}$, Guimaranes J. ${ }^{\mathrm{e}}$, Alonso I. ${ }^{\mathrm{b}}$, Silveira I. ${ }^{\mathrm{b}}$, \\ Sequeiros J. ${ }^{b}$, Marques Neves J. ${ }^{f}$, Serrano P. ${ }^{\mathrm{f}}$, Carolina S.M. ${ }^{\mathrm{b}}$ \\ aHospital de San Sebastian, CHEDV, Portugal; bUniversidade do \\ Porto, Portugal; ' ${ }^{2}$ ospital de Santo Antonio, CHP, Portugal; \\ dHospital Maria Pia, CHP, Portugal; 'British Hospital XXI, \\ Portugal; ${ }^{\mathrm{P}}$ Ministerio da Saude, Portugal
}

Background: Epidemiological data on the hereditary ataxias (HCA) and spastic paraplegias (HSP) are scarce. The precise clinical and genetic diagnosis of many affected families is uncertain. Our aim is to estimate the prevalence of HCA and HSP and describe some of the main clinical groups found in a Portuguese population-based survey.

Methods: This study covered all Portuguese regions, sequentially, throughout 1994-2004. Independent prevalence days were defined for each region Multiple sources were used to identify patients, including clinical files search, active call for the collaboration of neurologists and geneticists, but relying mainly in the active survey of general practitioners. Neurologists from the research evaluated all the patients using defined inclusion criteria. The clinical and genetic investigation of the included families extended until 2010.

Results: During the study 368 health centres were contacted, $86.1 \%$ cooperated. From 2724 suspected patients, 1336 patients were diagnosed with HCA/HSP. The estimated overall prevalence was 12.9:100, 000 for a population of 10, 322 millions. The prevalence of HCA was 5.6:100, 000 for dominant and 3.3 for recessive. The HSP are less prevalent, 2.4:100, 000 for dominant and 1.6:100, 000 for recessive. Machado-Joseph disease (SCA3) is te most prevalent hereditary ataxia, especially in the Azorean islands, almost eight-fold that in the whole country; the cluster in the island of Flores showed a prevalence of 10.4:1, 000. In the mainland, the cluster in the Tagus Valley had a prevalence of 1.4:1, 000. Friedreich ataxia and ataxia with oculomotor apraxia (AOA) were the most prevalent recessive HCA, 1.0:100, 000 and 0.4:100, 000 respectively. The most prevalent HSP forms were SPG4 and SPG3, $0.91: 100,000$ and $0.14: 100,000$ respectively. The genetic cause has not been identified in $39.6 \%$ of the patients.

Conclusions: This population-based survey covered all the Portuguese territory and mobilized the majority of family doctors and health centres. It is probably the largest ever performed in the field of HCA and HSP. Most population-based studies yielded similar overall prevalence values. Lower figures are often found in hospital-based or genetic centres studies, suggesting the great advantage of population-based surveys and of using all possible sources of information Several novel genes were discovered. New disease clusters and multiple undiagnosed families were identified, setting the basis for assistance, prevention and counselling programs. 


\section{Session 09: Neuropharmacology}

85

\section{Survey of Pharmacotherapy in Pediatrics with Autistic Disorder}

Nakagawa E.

National Center Hospital, National Center of Neurology and

Psychiatry, Japan

Background: The most main core symptoms of autistic disorder are impairment in behavioral manifestations or maladaptive behaviors. Medication can be an effective for autistic symptoms of childhood but its use should be based on a comprehensive psychiatric evaluation and treatment plan. The aim of this study was to evaluate psychotropic medication use for children with autistic disorder in Japan.

Methods: We surveyed the circumstances of drug therapy in autistic symptoms for 1003 pediatric neurologist and 150 doctors certified by the Japanese Society for Child and Adolescent Psychiatry during the period from October 2010 to May 2012 in Japan.

Results: Response rate was 73\% (626/1153) for our questionnaire survey and these 54\% (457/626) doctors been answered were worked on drug therapy. We have found that the $67 \%$ of pediatric neurologist were started to treat with pharmacotherapy from preschool age, and $90 \%$ of these doctors from school age. They had prescribed psychotropic medication to sleep disorder and irritability up to 3-4 years old, and to impulsiveness, hyperactivity, selfinjury, and harm to others up to 5-6 years old. Risperidone was most commonly prescribed medication for irritability and selfinjury and harm to others. Melatonin and risperidone were most commonly administered drugs for sleep disorders from one year old. For impulsiveness, risperidone was most prescribed in preschool children, methylphenidate in school-age children. Methylphenidate was also most commonly administered for hyperactivity and inattention in preschool children. Risperidone had been administered $0.3 \sim 1.0 \mathrm{mg}$ /day for preschoolers, $0.6 \sim 2.4$ $\mathrm{mg} /$ day for school-age children in Japan.

Conclusion(s): We should need to establish treatment guidelines for the symptoms of autistic children in Japan.

\section{4}

\section{Neurotrophin Delivery Using Nanotechnology \\ Angelova A. ${ }^{\mathrm{a}}$, Angelov B. ${ }^{\mathrm{b}}$, Lesieur S. ${ }^{\mathrm{a}}$ \\ ${ }^{a}$ CNRS UMR8612 Institut Galien Paris-Sud, Univ Paris-Sud 11, France; 'Institute of Macromolecular Chemistry, Academy of Sciences of the Czech Republic}

Background: Neurotrophins are becoming leading therapeutic candidates but their therapeutic use is restrained by the fragility and rapid degradation in the biological medium. The neurotrophin encapsulation in nano-sized vector systems is anticipated to overcome the problems resulting from the unfavorable pharmacokinetics in the cerebral circulation. Towards the aim to make use of nanotechnology for neurotrophin delivery, we performed a critical comparison of the nanoparticulate carriers, which may provide controlled release profiles and prolonged presence of the therapeutic proteins in the circulation as well as receptor targeting. The purpose of this study was to design and characterize LC lipid nanoparticles (NPs) suitable for transport and delivery of neurotrophic molecules.

Methods: The NPs were characterized by small-angle X-ray scattering (SAXS) and quasi-elastic light scattering (QELS).

Results: Among the soft-matter nanocarriers (liposomes, lipid nanocapsules, solid lipid nanoparticles, nanoemulsions, polymeric micelles, dendrimers, nanospheres, nanogels, and nanoporous scaffolds), we focused on liquid-crystalline (LC) lipid nanoparticle formulations for neurotrophin delivery. Sterically stabilized nano-dispersed lipid systems were prepared from a PEGylated liquid-crystalline lipid phase. Such lipid nanovectors, derived by self-assembly, are of ongoing interest thanks to their biocompatible compositions and the relatively low energy input required for their manufacture. The stability of the prepared PEGylated LC NPs was investigated. The obtained structures provide milti-compartment reservoirs for therapeutic molecules.

Conclusion(s): These advantages make the nanostructured LC lipid systems very interesting carriers for the administration of neurotrophins.

\section{Session 010: Neuropsychology}

42

\section{Neuropsychological Profiles of 5-Year Ischemic Stroke Survivors by Oxfordshire Stroke Classification}

Barker-Collo S. ${ }^{a}$, Starkey N. ${ }^{\mathrm{b}}$, Lawes C. ${ }^{\text {, }}$, Valery F. ${ }^{\mathrm{c}}$, Varsha P. ${ }^{\mathrm{a}}$

aUniversity of Auckland, New Zealand; 'The University of Waikato, New Zealand; 'AUT University, Auckland, New Zealand

Background: The neuropsychological literature typically examines stroke outcomes by hemisphere of lesion, while the medical literature provides classifications more closely linked to circulatory distribution impacted by stroke. This paper examines profiles of cognitive function by hemisphere and by Oxfordshire Community Stroke Project(OCSP) stroke classification.

Methods: This study included a sample of 315 5-year ischemic stroke survivors. Assessment included tests of verbal memory, visual memory, word finding/verbal fluency, abstract visual reasoning, executive functioning, and speed of processing.

Results: The sample produced average scores within 1SD of the normative mean on tests of abstract visual reasoning, verbal memory, and visual recall. Impaired performances were observed 
for executive function and processing speed. Profile analysis revealed no significant differences in overall cognitive performance or in the profile of performance across measures by hemisphere of lesion. However, groups defined by OCSP categories produced significantly different cognitive profiles. Post hoc analyses indicate those with Posterior stroke performed best overall on all tests except the Stroop dots trial, while those with Total Anterior stroke produced significantly worse scores on tasks requiring visual abstract reasoning (Block Design, Rey Figure Copy), word finding (Boston Naming Test), and processing speed (Stroop Dots, Trails A).

Conclusion(s): OCSP stroke subtypes differed significantly suggesting this classification system is of greater use than hemisphere of lesion in predicting likely post-stroke cognitive outcomes.

\section{Session 011: Neuropsychiatry}

\section{4 \\ Italians and Panic Attacks: Incidence and Knowledge of the Disease}

Pezzella F.R., Sorrentino R.

ISNEG Istituto Neuroscienze Globali, Italy

Background: Only limited information exists about the epidemiology of DSM-IV panic attacks (PAs) and panic disorder (PD), as well as on community awareness, kwnoledge and attitude toward the disease. We developed a computer assisted survey to estimate incidence of panick attacks in the Italian Population.

Methods: To enhance the survey discrimination accuracy a stepwise approach has been chosen. In the first stage assessment, respondents were asked a checklist of symptoms containig distractors and three panic disorders constellations of symptoms; in second stage respondents were openly asked about their possible previous experience of panic attacks; in the third phase respondents who were positive at the screening phase (first survey stage) received an explanation and information on reported symptoms and panic disorder and were asked to confirm or not if they ever suffered PD or PAs. The fourth step consisted of a follow up check to verify feedbacks consistency.

The survey has been administerd with computer assisited web interview modality.

Results: The survey was conducted twice: in 2009 on a sample of 1017 Italian respondents aged 18-60 years, and in 2011 in a sample of 833 aged 18-60 years respondents who were residents in the Rome metropolitan area. According to surveys projection incidence of PD with or without agorafobia and of PAs is respectively $14 \%$ and $7 \%$ in the Italian population and $15 \%$ and $11 \%$ in the Rome metropolitan area. Direct cost of the disease has been estimated 1800 euro per year per patient at a National level, 1470 euro in the rome metropolitan area.
Conclusion(s): This survey describes how PD and PAs are perceived in the Italian population. In this respect the survey represents a real world picture, altough with limitations due to possible overestimation of disease incidence.

\section{3}

\section{Prazosin, a New Treatment for the Post-Traumatic Stress Disorder?}

Rousseau P.F., Simon P.Y., Clervoy P.

Hôpital d'Instruction des Armées Sainte Anne, Toulon, France

Background: Post-Traumatic Stress Disorder affects 10\% of soldiers leaved waging war. It doesn't exist a standart treatment for PTSD. The selective serotonin reuptake inhibitors (SSRIs) are considered as the first-line pharmacological treatment for PTSD. However response rate rarely exceeds $60 \%$ and less than $20-30 \%$ of the patients achieve full remission. Excessive brain responsiveness to norepinephrine particularly appears to play a significant role in PTSD. Prazosin is a brain active alpha-1 adrenergic receptor antagonist used to treat hypertension and benign prostatic hypertrophy. We present a review of studies using prazosin as treatment for PTSD.

Methods: We search all clinical studies using prazosin to treat PTSD in Pubmed until june 2012. We find only five studies and we present the main results.

Results: At least 99 patients were included in the five studies from 2004 until 2008. Patients have received a civilian trauma for $29 \%$ compared to a war trauma for $71 \%$. Prazosin significantly (p $<0.005$ ) decreases trauma nightmares, distressed awakening, avoidance, sleep disturbance, hyper-arousal, re-experiencing and improve global clinical status compared to placebo in all studies. Blood pressure at the end of the treatment did not differ between prazosin and placebo conditions in each study case.

Conclusion(s): Prazosin appears as an outcome to treat patient with PTSD without major adverse effect. But studies published have few patients for a short time of follow-up. A longer duration study in larger samples would be necessary to confirm the preliminary findings.

\section{5}

\section{Unipolar Depression in Elderly Treated with Home Visits: Follow-Up Study}

Flemming N. ${ }^{\mathrm{a}}$, Kjeld A. ${ }^{\mathrm{b}}$, Lolk A. ${ }^{\mathrm{b}}$

aUniversity Hospital of Copenhagen, Hvidovre, Denmark;

bUniversity Hospital of Odense, Denmark

Background: Depression in elderly is frequent. In nearly all psychiatric departments in Denmark, ambulatory care is delivered as outgoing teams doing home visits in the patients' home, either private or in nursing home. Newer studies have raised serious questions and diverging results on the efficacy of antidepressive medication $(\mathrm{ADM})$ in elderly patients. 
In this study we investigated if data with quality improvement system using clinical indicators were useful in evaluation of treatment efficacy in a naturalistic setting.

Material and Methods: In a period of two years we evaluated all consecutive patients referred to two geriatric psychiatric services for depressive symptoms. Patients with evident dementia were excluded. Standards for evaluation and treatment were developed according to quality of good standard treatment in Danish Health Authorities. Quality improvement system using clinical indicators was implemented in both departments. We trained in rating-scales for all staff members involved.

Demographic, social and medical data were registered. The patients were followed for up to two years and more, and evaluated at discharge. Number of visits, use of medication, Hamilton-6 item, and other ratings for depressive symptoms were registered. Statistics used were frequencies, two by two tables, correlations, logistic regression, and survival analysis.

Results: We evaluated 66 patients. 10 were excluded (dementia, death before evaluation, and lack of symptoms). The 56 remaining patients were followed up. At present time, not all patients are discharged from follow-up. Preliminary results show $87 \%$ females, on average 81 years old. Recurrent depressive disorder was found in $55 \%$. Average number of home visits was 5.3 (SD 2.85). Average Hamilton- 6 at first visit was 8.1 and at discharge visit was 3.0. The main other findings (time in waiting, ADMs used, and changes in ADM, regression analyses on various parameters etc.) are presented.

Discussion: In a naturalistic setting, we evaluated, which elements in the ambulatory home visit service were important in treating elderly patients for newly developed moderate to severe depressive disorder episode.

Conclusion: Preliminary results indicated that home visits by psychiatrically trained and experienced nurses were the single most effective factor with influence on the outcome on depressive symptoms in elderly patients. ADM was of lesser importance. Side effects were in the doses used here of insignificant importance. This study indicates that a larger study with double-blind design of a clinical trial with placebo arm should be conducted.

\section{Session 012: Basic and Translational Neuroscience}

\section{6 \\ Developmental Effects of Neonatal Anesthesia with Sevoflurane}

Martynyuk A.

University of Florida, USA

Background: There is a high level of concern that exposure of newborns to general anesthesia may alter the course of brain development. Although strong evidence for age-dependent devel- opmental effects of general anesthetics is provided by numerous laboratory investigations, the results of human retrospective epidemiological studies are less conclusive. The design of human epidemiological studies suffers from an incomplete understanding of the mechanisms of developmental effects of anesthetics, even in animal studies and a limited focus on pathophysiologic factors that may modify susceptibility. This study was designed to contribute to resolving these issues.

Methods: We sought whether subjects with pathophysiological conditions that are characterized by elevated levels of aldosterone (preterm birth, urinary infection and many others) have increased susceptibility to the side effects of neonatal anesthesia with sevoflurane.

Results: Sevoflurane, administered to postnatal day 4-6 (P46) rats, resulted in seizure-like EEG patterns, increased levels of aldosterone in the blood, activated caspase- 3 in the cerebral cortex and delayed reduction in the prepulse inhibition (PPI) of the acoustic startle response. Exogenous aldosterone further increased sevoflurane-induced seizure-like activity, levels of activated caspase- 3 and disruption of PPI of startle, while spironolactone, a mineralocorticoid receptor inhibitor, caused opposite effects.

Conclusion(s): These results suggest that findings by human epidemiological studies could be more significant if the study subjects were restricted to those with pathophysiological conditions characterized by excess aldosterone at the time of exposure to sevoflurane.

\section{Session 015: Health Economics}

\section{6 \\ Preventing Alzheimer Disease: Is There a Place for Cost-Effective Interventions? Dynamic Modeling Applied to a Pan European Analysis}

Roze S. ${ }^{\text {, }}$ Marty R. ${ }^{\text {a }}$, Vainchtock A. ${ }^{\text {a }}$, Krolak-Salmon P. ${ }^{\text {b }}$

aHEVASAS, France; bHospices Civils de Lyon, France

Background: With population aging across European countries, Alzheimer's disease (AD) represents an increasing burden for societies and health care payers. The present study assessed the health-economic potential benefits of hypothetical interventions in preventing $\mathrm{AD}$ across 9 European countries.

Methods: A 3-state dynamic Markov model was developed in a population of $65+$ year old patients. Age-specific conversion rates from No $\mathrm{AD}$ to $\mathrm{AD}$ were retrieved from a European study. On top of the non-specific mortality rates, an adjustment factor was applied to patients with $\mathrm{AD}$. Annual costs data for $\mathrm{AD}$ were retrieved for 9 major European countries including France, Germany and the UK. These costs (societal perspective) ranged from $€ 9,856$ in Ireland to $€ 36,885$ in the UK. Two preventing AD interventions were simulated, based on relative risk reductions (RRR) of 0.8 and 0.5 . 
Results: In order to be break-even, annual costs of interventions ranged from $€ 90$ in Denmark, Ireland and Sweden to $€ 335$ in the UK and from $€ 230$ up to $€ 831$ in the same countries, respectively for interventions with RRR 0.8 and 0.5 . For a given willingness to pay of $€ 50,000$, interventions were cost-effective in France at $€ 380$ and $€ 970$, in Germany at $€ 290$ and $€ 770$, respectively for RRR of 0.8 and 0.5 . For these 2 interventions, average per patients expected savings ranged from $€ 1,859$ and $€ 4,829$, respectively for RRR 0.8 and 0.5, in Belgium. The demographic projections led in France to a reduction of $\mathrm{AD}$ prevalent patients of 25, $295(0.8)$ and $66,334(0.5)$ cases. This translated into annual savings in France of $€ 559$ million and $€ 1.465$ billion, respectively for RRR of 0.8 and 0.5 .

Conclusion(s): These analyses demonstrate the large potential health-economic benefits of $\mathrm{AD}$ preventing interventions. The results are robust across 9 major European countries and should encourage intervention development and financing.

\section{Session 018: Public Health}

\section{0 \\ Knowledge of Stroke Warning Signs and Risk Factors in Rural Population of India}

SoahamD., Devangi D., Udgeeth T., Jyoti M.

Pramukhswami Medical College and Shree Krishna Hospital, India

Background: Stroke is a leading cause of death and disability. Limited data from India suggest poor knowledge about stroke warning symptoms and risk factors in urban population. The awareness regarding these in rural population is likely to be lesser than the urban population. This study intended to study awareness of stroke warning signs and risk factors in a rural based setting.

Methods: Amongst persons visiting Shree Krishna hospital, a rural teaching hospital sit in Karamsad in Western India, 656 randomly selected persons were asked to respond to structured questionnaire, administered in written/ verbal format, by trained persons. Questions assessed knowledge of stroke warning signs, risk factors and personal risk factors amongst the interviewees.

Results: 230 [35.06\%] described limb weakness, 99[15.09\%] described numbness, 65[9.90\%] described headache, 20[3.04\%] described difficulty in speaking as a warning signal of stroke. None of the participants had knowledge that dizziness or imbalance or difficulty in vision could be a warning signal of impending stroke. 360[55\%] had no knowledge about any of the warning signs. None of the participants had correct knowledge about all the warning signals.

Amongst participants, only 223 [33.99\%] were aware of at least one of the risk factors of stroke. Awareness about individual risk factors was 85 [12.9\%] hypertension, 74 [11.2\%] diabetes, 39 [5.94\%] smoking, 15 [2.2\%] alcohol, 33 [5.03\%] dyslipidemia, 14 [2.13\%] sedentary life, $5[0.77 \%]$ age.

Conclusion: There is severe lack of knowledge of stroke warning signs and risk factors, thus calling for urgent need for public campaigns for stroke awareness, especially in rural India. 


\section{Session P1: Prevention}

\section{9}

\section{New Era in Understanding of Atheroaclerosis and Plaque Morphology. From Cholesterol Through Lipoproteins, LDL/HDL-Subfractions to Specific Vascular Inflammatory LP-PLA2 Marker}

\section{Bartko D.}

\section{Central Military University Hospital, Slovakia}

Background: Some years ago new data have been published, showing that no cholesterol and LDL-lipoproteins but specific vascular inflammation marker could be responsible for dvelopment of atherosclerois.

Aim: To analyse various parameters of arterial wall and LP-PLA2 in AH, CAD, iCI and controls. Prospective multicenter, multidisciplinary study.

Methods: Material: 566 subjects divided into four groups: 1 . $\mathrm{AH}(\mathrm{n}=117)$, mean age $60.0+/-11.2$, men $48.3 \%, 2$. CAD ( $\mathrm{n}=$ 95), mean age $70.8+/-1.8$, men $51.5 \% 3$. iCI $(\mathrm{n}=102)$, mean age73.4+/-9.9, men 54.2\%, 4. Controls $(\mathrm{n}=102)$, mean age $47.5+/-18.5$, men $53,7 \%$.

In all pts:NIHSS, BI, mRS, LP-PLA2, lipid spectrum, other biochemical-hematological parameters, CT/MRI (ischemic volume, a x b x c x 0.5), Stiffness, IMT, long-term ECG and BP monitoring. Student-test, Pearson-Spearman-Kandell, Whitney U tests.

Results: Development of atheroslerosis started by $\mathrm{t}$-cholesterol, continued by lipopreteins, LDL/HDLsubfractions and at present new specific inflammatory vascular enzyme(LP-PLA2) is on horizon. Study showed :LP-PLA2 significantly higher in AH $(219.01+/-27.64)$, CAD $(234.02+/-51.58)$ and iCI groups (259.2+/-86) comparing to controls $(183,27+/-25.2)$. No significant correlation between LP-PLA2 and T-chol. Stiffness, IMT significantly higher in AH/CAD/iCI. Significant correlatation between plaque morphology-ulcer and LP-PLA2.

Conclusions: Study showed significant changes in LP-PLA2, stifness, IMT plaque morphology not only in iCI/CAD but also in $\mathrm{AH}$ (candidates of $\mathrm{iCI}$ ) comparing to controls. It could signalized new era in understanding mechanisms of atherosclerosis. Lp-PLA2 is a key inflammatory marker, specifically linked to plaque inflammation and plaque rupture.

Supp.by Intern/gov.grants ITMS26220220099, ITMS26220153, APVV0586-06, LPP0186-06.

\section{2}

\section{Are the Changes in Baroreceptor Sensitivity Age- Related in Healthy Subjects, Arterial Hypertension, Coronary Artery Diseases and Ischemic Cerebral Strokes?}

Bartko D.

Central Military University Hospital, Slovakia

Background: Age-related changes in barorecepor sensitivity(BRS)in healthy subjects are poorly documented, and literary data of its complications $(\mathrm{AH}, \mathrm{CAD}, \mathrm{iCI})$ are lacking.

Aim: To evaluate age-related BRS changes in $\mathrm{AH}$, and its complications (CAD, iCI)comparing to healthy controls.

Methods: 746subjects, four groups: 1 AH, $\mathrm{n}=226$ ), mean age $60,85+/-9.99$, men $48.3 \%, \mathrm{BMI}=29.2+/-4.8,2 . \mathrm{CAD}, \mathrm{n}=177)$, mean age $70,96+/-9.8$, men $42.5 \%), \mathrm{BMI}=28.8+/-3.8,3 . \mathrm{iCI}, \mathrm{n}=$ 213), mean age $66.8+/-10.7$, men $52.7 \%$, BMI $27.6+/-4.5,4.4$. controls $(\mathrm{C}, \mathrm{n}=130)$, mean age $50.74+/ 11.3$, men $44,2 \%$, BMI 23.9+/-4.4. Three age-related subgroups: 1st < 40yr, 2nd: 41-60yrs, 3rd > 60yrs. Diagnosis: CT/MRI(volume), USG, NIHSS, lipid spectrum, inflammation markers, BRS, IMT, stiffness. Pearson-Spearman-Kandell, Student, Whitney U tests.

Results: We have previously documented BRS decreasing in $\mathrm{AH}, \mathrm{CAD}, \mathrm{iCI}$ pts Regarding age-relating BRS changes, the study showed following findings. Controls: statistically significant differences between 1 st vs 2 nd age-group ( $p<.001)$, 1st vs $3 \mathrm{rd}$ ( $\mathrm{p}<$ .0001 )and 2 nd vs 3 rd age-group $(\mathrm{p}<.02)$. AH: the same trend of differeneces. CAD: Significant changes betwen 2 nd and 3rd group. iCI: Differences similar to AH, but significance with higher power. Results documented strong negative correlation between increasing age and decreasing BRS $(\mathrm{p}=.001)$. Significant nonlinear correlations between BRS and stiffness, IMT, carotid plaques.

Conclusions: 1 . The largest prospective, multicenter, multidisciplinary study documented significant lower BRS values in all three groups of pts $(\mathrm{AH}, \mathrm{CAD}, \mathrm{iCI}) .2$. Changes are starting already before $40 \mathrm{yrs}$ of age and are most significant in age $>60 \mathrm{yrs}$. 3 . Close negative correlations between arterial stiffness, IMT and carotid plaques was documented only in the $2 \mathrm{nd}$, and $3 \mathrm{rd}$ group of pts. Results can contribute to implementation to practice and future research.

Supp, by gov/EUgrants APVV0586-06, ITMS26220220099, ITMS2622022153, LPP0186-06.

\section{KARGER \\ Fax +41613061234 \\ E-Mail karger@karger.ch}

๑) 2012 S. Karger AG, Basel www.karger.com
Accessible online at: www.karger.com/ned 
251

\section{Ideal Cardiovascular Health Metrics on the Prevalence of Asymptomatic Intracranial Artery Stenosis}

Zhang Q., Shufeng Z., Chunxue W., Heng Z., Liheng B., Zhao X.

Beijing Tiantan Hospital, China

Background: Intracranial Artery Stenosis (ICAS) is one of the most common causes of ischemic stroke among Asian patients. Previous studies showed a strong relationship between the number of ideal cardiovascular health metrics and lower risk of stroke. However, little is known regarding its relationship with ICAS.

Methods: A random sample of 5, 412 participants (selected from Kailuan Study as a reference population) aged 40 years or older $(40.10 \%$ women) free of stroke, transient ischemic attack, and coronary disease was enrolled in the Asymptomatic Polyvascular Abnormalities Community study (APAC) in 20102011. We collected information on cardiovascular health metrics (including smoking status, body mass index, dietary intake, physical activity, blood pressure, and serum concentrations of total cholesterol and fasting blood glucose) and assessed the presence and severity of ICAS with transcranial Doppler ultrasonography. We then investigated the relationship between ideal cardiovascular health metrics and the prevalence of ICAS with logistic regression.

Results: The multivariate-adjusted odds ratios for ICAS with adherence to 0 or 1 (reference), 2, 3, 4, 5, and 6 or 7 ideal health metrics were $1,0.76,0.55,0.49,0.43$, and 0.36 ( $\mathrm{p}$-trend $<0.0001$ ) respectively after adjusting for age, sex, and other covariates. Similar inverse associations were observed in different age groups, both male and female ( $\mathrm{p}$-trend $><0.05$ for all).

Conclusions: We observed a clear inverse dose-response relationship between the number of ideal cardiovascular health metrics and lower prevalence of ICAS in a Chinese population, which supports the importance of ideal health behaviors and factors in the prevention of ICAS.

\section{Session P2: Diagnosis}

\section{8}

\section{Different Characterisitic Phenotypes in Three Types} of Myasthenia Gravis

Oh Shin J.

University of Alabama at Birmingham, USA

Background: This study is done to characterize three types of MG.

Methods: We analyzed all the clinical, laboratory, and electrophysiological data among 235MG patients who were evaluated in the Neuromuscular Disease Clinic of the University of Alabama at Birmingham between May 2003 and January 1, 2008. A diagnosis of MG was made when there was fluctuating muscle weakness and at least one of four tests was abnormal: AChR-Ab, MuSK-Ab, repetitive nerve stimulation (RNS) test, and single-fiber EMG (SFEMG). MuSK-Ab was checked in all AChR-Ab negative patients.

Results: There were 15 cases of MuSK-MG, 59 cases of DSN-MG, and 161 cases of AChR-MG. AChR-Ab was positive in $69 \%$ of cases and MuSK-Ab, in 6\% of cases. MuSKMG was more common (14\%) in African Americans compared with whites (4\%). AChR-MG is characterized by male predominance, later onset, a fewer cases of ocular MG, and a higher association with thymoma. DSN-MG is characterized by a greater prevalence of ocular MG, milder forms of MG with less number of crisis, and fewer abnormalities in the RNS test. MuSK-MG is characterized by younger age at onset, severe and bulbar forms of MG, predominant facio-bulbar-neck weakness, and a poor response to edrophonium, anti-cholinesterase, and IVIG. Long-term-outcome showed no difference among three types of MG.

Conclusion(s): AChR-MG and DSN-MG are similar, with the exception of less severity in the latter. MuSK-MG has distinct clinical and electrophysiological features (in this section, importance of results of the study for practice and/or future research is briefly described).

\section{0 \\ Distinct Patterns of Spontaneous Speech Decline in Probable Alzheimer's Disease}

Jung-Wan $K$.

Daegu University, South Korea

Background: Detecting probable Alzheimer's disease (AD) at an early stage is very crucial in modifying the disease and conducting an initial drug therapy for more effective management. Thus, in this study, we focused on identifying the speech features, which allows us to distinguish between patients with probable $\mathrm{AD}$, one of the neuropsychological deficits of $\mathrm{AD}$, and normal individuals.

Methods: This paper reports on four speech parameters of picture description in three stages of $\mathrm{AD}$ (probable, mild, and moderate) compared to education and age-matched normal controls. Four parameters of speech was measured in Korean native speakers with AD and normal elderly: speech tempo, hesitation(sec), rate of articulatory errors, and rate of grammatical errors.

Results: The results revealed significant differences in most of these speech parameters among the four groups (probable, mild, moderate $\mathrm{AD}$ and normal controls). Additionally, the clearest difference between the normal controls and the probable $\mathrm{AD}$ involved the speech tempo $(\mathrm{F}=15.645, \mathrm{p}<.001)$, which was significantly lower in the later stage in $\mathrm{AD}$.

Conclusion(s): Spontaneous speech in $\mathrm{AD}$ is affected by dementia. The domain affected by the disease varies depending on the degree of severity. By evaluating speech tempo parameters 
more sensitively during a picture description task, we were able to distinguish probable $\mathrm{AD}$, which was difficult to detect with cognitive decline during routine medical practice using interviews. Prospective assessment of spontaneous language skills with a picture description task is useful to detect those subtle spontaneous speech impairments caused by $\mathrm{AD}$ even at an early stage of the disease.

11

\section{Anticoagulants Followed by Long Term Use of Azathioprine and Aspirin in Childhood Primary Arterial Stroke}

MalikM.A.

BrainAssociates Lahore, Pakistan

Background: Primary anggiitis of CNS in children is a poorly understood entity.

Methods: Over the period of 2 years, 68 patients with acute cAIS were admitted and ischaemic strokes, who presented within 14 days of onset. Patients with ischaemic infarcts were treated with anticoagulants at least for 04 weeks and this was followed by long term use of Aspirin and Azathioprine. Patient were followed in Hospital based cohort study at single center and were systemically assessed for clinical presentation, classification of cAIS, adverse effects of the therapy.

Results: 68 children with cAIS (boys 62\%, girls 38\%) with mean age of 8.5 years, were enrolled in this study. Motor deficit (70\%); headache $(64 \%)$ and fever $(20 \%)$ were the commonest symptoms, whereas, hemiparesis (60\%); seizure $55 \%$ (focal $35 \%$, generalized 20\%); and decreased conscious level (30\%), were the commonest neurological findings. Neuroradiological findings of head revealed; ischemic strokes 50 (73.5\%); hemorrhagic strokes $10(14.7 \%)$ and ischaemic- haemorrhagic lesions 8(11.8\%). Conventional angiography and/or MRA revealed that at the time of admission $51(51 / 68,75 \%)$ of the cohort had non-progressive (obliterative) and $17(17 / 68,25 \%)$ had evidence of progressive arteriopathy. No secondary hemorrhagic was documented among infarcts strokes who were treated with heparin and anticoagulants. Hospital outcome was as; survivors $56(81.5 \%)$ and deaths 12 (18.5\%), 40patints discharged on long term oral aspirin and 14 children of these 40 were commenced also on Azathioprine and are on follow-up. The Neurological findings among 56 survivors were; normal 20\%; minor disabilities $25 \%$; moderate disabilities $20 \%$ and severe disabilities $35 \%$.

Conclusion(s): Characteristic features at diagnosis can be used to predict later progression, to identify a distinct high-risk cPAIS cohort, and to help guide selection of patients for immunosuppressive therapy.
18

\section{Manganese (Mn) Dependent Neurological Disorders and $\mathbf{M n}$-Speciation}

Bernhard M.

Helmholtz Zentrum München, Germany

Background: For humans manganese $(\mathrm{Mn})$ is an essential trace element, but at higher doses a neurotoxic metal. Chronic Mn exposure is affecting the central nervous system. Mainly occupational $\mathrm{Mn}$ overexposure leads to accumulation in brain and has been shown to cause progressive, permanent, neurodegenerative damage, resulting in syndromes similar to idioparthic Parkinson 's disease. $\mathrm{Mn}$ is transported by an active mechanism across neural barriers (NB) finally into brain, but to date modes of Mn neurotoxic action are poorly understood.

Methods: This paper investigated the relevant $\mathrm{Mn}$-carrier species which are responsible for a widely uncontrolled transport across NB. Mn speciation in paired serum / cerebrospinal fluid (CSF) samples was performed by SEC-ICP-DRC-MS and CZEICP-DRC-MS.

Results: In serum protein-bound Mn-species like $\mathrm{Mn}$-transferrin (Mn-Tf) were dominant, which had main influence on total $\mathrm{Mn}$ in serum if Mntotal was $<1.5 \mu \mathrm{g} / \mathrm{L}$. Above 1.6 $\mu \mathrm{g} / \mathrm{L}$ Mntotal in serum was ruled by the increasing concentration of Mn-citrate (Mn-Cit). In parallel Mntotal and Mn species were determined in CSF samples. It turned out that Mntotal from CSF was ca. half of Mntotal in serum, Mn-Tf even only about $10 \%$ compared to serum, however in turn, Mn-Cit was enriched by a factor of 4-5 in CSF. If Mntotal concentration in serum was > 1.6 $\mu \mathrm{g} / \mathrm{L}$, then $\mathrm{Mn}$-Cit was the leading Mn-species in serum, and moreover was significantly influencing the Mntotal concentration and $\mathrm{Mn}$-Cit concentration in CSF.

Conclusion(s): It can be concluded that elevated Mn-Cit in serum could be a valuable marker for increased Mn concentration in CSF (and brain), i.e. a marker for elevated risk of Mn-dependent neurological disorders such as Manganism (Mn dependent Parkinsonism-like disease) in occupational health.

\section{9}

\section{ROS and Brain: Se-Speciation Investigations}

Bernhard M.

Helmholtz Zentrum München, Germany

Background: The beneficial health effects like protection against reactive oxygen species (ROS) of Se-species initiated Se-speciation for a deeper insight into Se-metabolism and transport. This is of paramount importance for Se transport across neural barriers (NB).

Methods: Se-speciation was performed in paired serum and cerebrospinal fluid (CSF) samples by SAX-ICP-DRC-MS.

Results: Serum samples had $58 \mu \mathrm{g} \mathrm{Se/L}$ and CSF 0, $86 \mu \mathrm{g} \mathrm{Se} / \mathrm{L}$ (median). Prominent Se species were SeP, GPx, TrxR, Se(IV) and Se-HSA. Other Se-species were monitored only in few samples close to LoD. 
Relationships were calculated between Se-species from serum and CSF to evaluate Se-species passage across NB: SeP-serum ruled total Se-serum when the latter was $>65 \mu \mathrm{g} / \mathrm{L}$, however, SePP-CSF appeared independent on SeP-serum. For Se-HSAserum vs Se-HSACSF a weak linear relationship was found ( $\mathrm{r} 2=$ 0.1722 ), but for Se-enzymes protecting against ROS higher $\mathrm{r} 2 \mathrm{val}$ ues were calculated: GPx-serum vs GPx-CSF had r2 $=0.3837$ and TrxR-serum vs TrxR-CSF reached $\mathrm{r} 2=0.6293$, confirming influence of TrxR-serum on that in CSF.

Additionally, the ratios (CSF/serum) of Se-species were drawn. For Se-HSA the ratio was $3.8^{\star} 10-3$, fitting to molecular mass dependent restriction at NB. Significantly increased ratios were seen for anti-oxidative Se-enzymes: TrxR had $21.4^{\star} 10-3$ and GPx $8.3^{\star} 10-3$. This cannot be explained by molecular size dependence, as GPx is larger than Se-HSA and the TrxR ratio is increased much more than regarding the somewhat smaller molecular mass.

Conclusion(s): These findings allow hypothesizing, whether for these two anti-oxidative Se-enzymes a facilitated diffusion across NB exists or whether they might be also expressed in brain.

\section{3 \\ Delayed Cognitive Impairment After Electrical Injury: Neuropsychological and MR Spectroscopy Findings in a 48-Year-Old Woman}

Gasser A.-l. ${ }^{a}$, Simko M. ${ }^{b}$, Rossier P. ${ }^{a}$

${ }^{a}$ Hôpital fribourgeois HFR Billens, Switzerland; ${ }^{b}$ Centre d'Imagerie Médicale, Switzerland

Background: Electrical injury (EI) represents a potentially permanent source of cognitive and emotional symptoms, which can interfere with a patient's ability to return to work. While delayed onset of cognitive dysfunction is frequently reported in the literature, few cases have been published that document the progressive neuropsychological manifestations of EI. Furthermore the pathogenesis of these deficits remains poorly characterised and controversial. Structural brain imaging frequently shows no abnormalities and the patients are therefore often considered as suffering from psychiatric pathology, or malingering in order to seek compensation.

Methods: We report the case study of a 48-year-old female who suffered a low-voltage EI with a short loss of consciousness of 1 minute. She was hospitalised during 3 days without complications except a mild rhabdomyolysis. She underwent neuropsychological evaluations at 1, 4 and 19 months post-injury, as well as structural MRI at 2 months and MRI with MR spectroscopy at 19 months.

Results: Neuropsychological pattern showed mild deficits in executive functions and attention at 1 month, followed by a normalisation of neuropsychological tests at 4 months, and a global cognitive dysfunction at 19 months, which was reported as progressive by the patient and her family, with severe impairment in memory, executive functions and attention at examination. Structural MRI was within normal limits, both at 2 and 19 months, but MR spectroscopy showed signs of widespread axonal destruction with important reduction of $\mathrm{N}$-Acetylaspartate (NAA) and diffuse intraparenchymal lipid accumulation at 19 months post EI.

Conclusion(s): This case illustrates the early improvement of initial mild neuropsychological deficits, followed by a delayed progressive cognitive decline that may occur within months following EI. It also shows the utility of MR spectroscopy to support the presence of a brain dysfunction. Moreover it highlights the importance of long term follow-up with patients after EI.

\section{9 \\ GLUT-1 Deficiency Syndrome or De Vivo Disease: A Case Report}

Ahmane H., Abrous N., Arhab D.

Hospital Nedir Mohamed Tizi-Ouzou, Algeria

Background: GLUT-1 deficiency syndrome was first described in 1991 by De Vivo and al., approximately 100 GLUT-1 DS patients have been identified in the United States and elsewhere in the world and only 5 cases were reported in the literature review with the mutation R $126 \mathrm{H}$.

Methods: We report a 5 months old male infant from a term pregnancy and a normal delivery, neonatal period without incidents, which has réccurents abnormal movements since the age of 1 month and type of eyelid blinking, nystagmus, with occasional brief loss of contact without loss of muscle tone that occurs prior to meals, duration ranging from 1 to 3 minutes on average every 15 days. The magnetic resonance imaging of the brain was normal, and the electroencephalographic (EEG) showed a multifocal spike-wave discharges.

Results: GLUT-1 deficiency was suspected in a lymphocytic meningitis with persistent hypoglycorrhachia, a cerebrospinal fluid /serum glucose ratio $<28 \%$. A deficit in GLUT-1 was confirmed by molecular analysis which showed a missense mutation pArg126His. The ketogenic diet was instituted the outcome was favorable by decrease in seizures.

Conclusion(s): The GLUT-1 deficiency is a rare affection, of recent identification and probably underdiagnosed.There were no obvious correlations between phenotype, genotype, or biochemical measures. The ketogenic diet produced good seizure control.

86

\section{Reliability of Dystonia Diagnosis}

Messina M. ${ }^{a}$, Regio V. ${ }^{a}$, Papantonio A.M. ${ }^{b}$, Beghi E. ${ }^{a}$

${ }^{a}$ Mario Negri Institute for Pharmacological Research, Italy;

${ }^{\text {b}}$ Ospedali Riuniti di Foggia, Italy

Background: The diagnosis of dystonia is mostly based on clinical assessment and may reflect personal background and experience. The aim of the study was to assess the inter-rater reliability of dystonia diagnosis among neurologists with differing professional experience. 
Methods: 29 case reports were selected from an Institute of Neurology by the caring neurologist (gold standard). 18 patients were affected by different forms of dystonia; 9 had other movement disorders; 2 were healthy controls. All subjects underwent video-recording including history and short neurological examination. 35 raters (12 general neurologists, 21 neurology residents and 2 experts in extra-pyramidal diseases) from different centers evaluated the videos and filled a questionnaire, identifying for each clinical condition the diagnosis and the affected body sites. Inter-rater agreement was tested with the kappa statistic. Sensitivity and specificity (dystonia: yes/no) were also calculated.

Results: Specificity and sensitivity were, respectively, 95.2\% and $66.7 \%, 76.3 \%$ and $75.2 \%, 84.6 \%$ and $71.6 \%$ for experts, general neurologists, and residents. The kappa values on dystonia diagnosis ranged from 0.30 to 0.46 . Agreement for experts and residents was moderate $(0.40-0.60)$ and for general neurologists was fair (0.20-0.40). Kappa values were highest for experts for cranial and laryngeal dystonia (0.61-1), but not for cervical dystonia (0.37).

Conclusion(s): The diagnosis of dystonia is difficult as agreement is at best moderate. Experts may report a lower level of accuracy than the other professional categories (false negatives 33\%). Experts reported an almost perfect agreement only for specific types of focal dystonia.

\section{1}

Trigeminal Neuralgia and Temporomandibular Joint Disorder - Differential Diagnostics in a Sample of Patients with Orofacial Pain

\section{Badel T. ${ }^{\mathrm{a}}$, Bašić Kes V. ${ }^{\mathrm{b}}$, Savić Pavičin I. , Zadravec D. ${ }^{\mathrm{b}}$, Kern J. ${ }^{\mathrm{c}}$, Krolo I. ${ }^{\mathrm{b}}$ \\ aschool of Dental Medicine, Croatia; ${ }^{b}$ Clinical Hospital Centre "Sisters of Charity", Croatia; 'School of Public Health "Andrija \\ Štampar", Croatia}

Background: The aim of this study was to evaluate accurate differentiating between temporomandibular joint (TMJ) disorder and trigeminal neuralgia (TN) in the sample of patients from subspecialist dental practice.

Methods: The study included 32 patients (group G-1) with TMJ disorder who were previously neurologically examined due to unconfirmed suspicions of a neurological origin of orofacial pain, 12 female patients (group G-2) with determined co-morbidity of TMJ disorder and TN, and 13 patients (group G-3) with only TN confirmed and the TMJ disorder ruled out. Clinical characteristics, pain intensity (in groups 1 and 2 to TMJs at mouth opening, in groups 2 and 3 pain related to TN) rated on a visual-analogue scale (VAS with range $0-10$ ) and maximal mouth opening capacity (in $\mathrm{mm}$ ) measured by gauge of all three groups were compared. The level of anxiety was evaluated by State-Trait Anxiety Inventory (STAI). Data were analyzed by t-test, chi-squared test, and correlation analysis.

Results: TMJ pain on the VAS scale for G-1 patients was 6.96 and for $\mathrm{G}-2$ patients 6.91 ( $\mathrm{p}=0.9325)$. TN related pain symptoms on the VAS scale were for G-2 patients 9.0 and for G-3 patients $7.98(p=0.2921)$. However, there was a statistically significant difference in the intensity of TMJ and TN related pain $(\mathrm{p}<0.001)$. Burning sensation in the mouth $(\mathrm{p}<0.0007)$ as well as toothache $(\mathrm{p}<0.002)$ were dominant symptoms in patients with TN (G-2 and G-3 patients). Maximal mouth opening was statistically significant $(\mathrm{p}=0.002$ ) between $\mathrm{G}-1 / \mathrm{G}-2$ groups and $\mathrm{G}-3$ patients: $39.34 / 39.77$ and $49.50 \mathrm{~mm}$. A positive correlation $(\mathrm{p}<0.05)$ existed between STAI 1 and STAI 2 scores independently within each group of patients.

Conclusion(s): Correct diagnosis is the key to managing facial pain of non-dental origin, which includes participation of several experts from the fields of dentistry, neurology and radiology.

\section{2 \\ Epilepsy: Electroencephalogram and Brain Maturation}

Bahbiti Y., Moutaouakil F. , Benazzouz B. ${ }^{\text {, }}$, Ouichou A. ${ }^{\text {, }}$ El Hessni A. ${ }^{\mathrm{b}}$, Mesfioui A. $^{\mathrm{b}}$

aDepartment of Neurology, Al Kortobi Hospital, Tangier, Morocco; 'baboratory of Genetics, Neuroendocrinology and Biotechnology, Unit of Nervous and Endocrine Physiology, Department of Biology, Faculty of Sciences, University Ibn Tofail, Kenitra, Morocco

The results of neurological examination are highly dependent on the maturation of the nervous system. The recording of brain electrical activity is an element of simple diagnostic and valuable, in particular to follow the normal and pathological maturation. A clinical study based on analysis of electroencephalograms of epileptic and normal subjects of different ages was carried out at the hospital Al-Kortobi of Tangier, Service of Neurology. Analysis of electroencephalograms showed, first, that during childhood the EEG is characterized by the gradual transformation of brain waves in time reflecting the different stages of brain maturation. On the other hand, there is a regional component of brain maturation in a posterior-anterior gradient chronological. Completion is set between 12 and 14 years. Brain maturation allowed therefore understanding the chronology of appearance of such épilepsie in such well determined age, motor seizures in newborns, and occipital epilepsy in the first months of life while the frontal and temporal epilepsy appear after the age of 2 years.

\section{4 \\ Standard CSF Dementia Markers Can Be Used for Differentiation of Molecular Subtypes in Sporadic Creutzfeldt-Jakob Disease}

Karch A., Gawinecka J., Hermann P., Ponto C., Zerr I.

University Hospital Goettingen, Germany

Background: Creutzfeldt-Jakob disease (CJD) is a rare neurodegenerative disorder. Six molecular subtypes of sporadic CJD 
(sCJD) have been identified. Classification is based on the methionine-valine polymorphism (MVP) at codon 129 of the Prion Protein gene and on two different types of prion protein (PrPSc type 1 and 2). Subtypes differ from each other with regard to disease course, survival time and MRI profiles. MVP can be analysed by genetic testing during lifetime, whereas information about PrPSc type is restricted to post-mortem autopsy.

Methods: We analysed csf dementia markers ( $t$-tau, p-tau, $\beta$-amyloid 1-42, $\beta$-amyloid 1-40, NSE, S-100) as well as csf desmoplakin from 400 CJD patients with confirmed molecular subtypes. Marker levels were compared between the different subtypes to identify biomarkers which might be helpful in subtype differentiation.

Results: Levels of all investigated markers varied across molecular subtypes. Subtypes with faster courses of disease were associated with higher levels of neuronal destruction markers. High levels of tau, NSE and S100 were more frequent in patients with PrPSc type 1. A combination of t-tau, NSE and S-100 was evaluated as a marker for subtype differentiation with promising results.

Conclusion(s): In this single-center study it could be shown for the first time that standard CSF dementia markers can be used in the differentiation of CJD subtypes. T-tau can be used as a biomarker for distinguishing between PrPSc Type 1 and 2 during lifetime when MVP is known. Moreover, a combination of standard CSF markers can be a useful diagnostic tool in the pre-mortem identification of the distinct subtypes. This will result in more detailed information for caretakers and families with respect to expected courses of disease and further medical support.

\section{8 \\ Rheumatoid Leptomeningitis: A Rare Diagnosis That Should Be Considered}

Koizia L. ${ }^{\mathrm{a}}$, Zielicka Z. ${ }^{\mathrm{b}}$, Koizia A. ${ }^{\mathrm{a}}$, Hamda A. ${ }^{\mathrm{a}}$, Rosenfelt K. ${ }^{\mathrm{a}}$

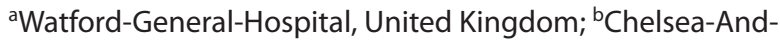
Westminster-Hospital, United Kingdom

Background: Rheumatoid arthritis (RA) is a chronic multisystem inflammatory condition with articular and extra-articular manifestations; with neurological complications being extremely rare.

Methods: A 66-year-old right-handed gentleman, with a 10 -year history of RA, presented with new onset generalized tonic-clonic seizures and associated right foot weakness. He underwent blood tests, MRI, lumbar puncture and finally a meningeal biopsy.

Results: Significant blood results included a raised CRP of $30.9 \mathrm{mg} / \mathrm{L}$, rheumatoid factor positive, negative angiotensin converting enzyme (ACE) and acid fast bacilli (AFFB). MRI revealed high signal within the meninges of the left frontal lobe on FLAIR imaging. Lumbar puncture identified no evidence of malignant cells, 9 white cells (100\% lymphocytes) and no organisms. Meningeal biopsy was performed and identified chronic inflammatory changes with no evidence of a neoplastic process, resulting in the diagnosis of rheumatoid leptomeningitis. The patient responded, clinically and radiologically, to prednisolone and has had no further seizures and no long standing neurological disturbances.

Conclusion(s): Leptomeningeal involvement in RA is rare but should be considered in patients when clinical and laboratory findings do not support the other differential diagnoses. Early consideration and diagnostic confirmation with a biopsy should be performed to allow effective initiation of treatment to prevent further neurological deficit.

\section{7}

\section{Neurological Manifestations of Acute Dengue Fever}

Ruikar D., Surath M., Jayalakshmi S., Varma R., Varalakshmi E.

Krishna Institute of Medical Sciences, India

Background: Dengue virus is generally considered as non neurotropic arbovirus, dengue viral infection is one of the commenest infection in tropics. Apart from case reports there is paucity of clinical and neuroradiological data available on neurological manifestation of acute dengue viral infection.

Methods: A prospective single centre study done at KIMS Hyderabad, during Nov 2007 to June 2011. Acute dengue virus infection defined as positive serology for IgM antibodies or PCR positive for dengue. CT/MRI brain, EEG and other necessary lab investigations were done. Patients admitted with acute dengue virus infection studied for neurological and radiological manifestations of acute dengue virus infection and followed for next 6 months.detailed statistical analysis done.

Results: Out of 281 patients with acute dengue fever/DHF who were admitted in our hospital during the study period, $31(11.08 \%)$ had neurological manifestations. Age was 14 to 68 years with mean age 36.6 years with M:F ratio 2:1. Mean GSC on admission was 11.8. headcache and altered conscioness was the most common clinical complaints followed by wide range of neurological manifetations. Diffuse slowing to be the commonest finding and there was no correlation of EEG findings on hospital stay or outcome[p-0.459]. On MRI T2 and flair thalamic hyperintesnities were the commenest radiological manifestation. Pontine T2 hyperintensity and blooming in thalamus was significantly associated with hospital stay[p $<0.005]$. GSC at admission has significant correlation with hospital stay, neurodeficits or condition at discharge \&outcome measures[p < 0.005] On 6 months follow up one patient died, two lost for follow up and one had new onset focal deficit at the end of six month.

Conclusion(s): Neurological manifestations of dengue present in wide range as encephalitis at one end and GBS or Myositis at other end. Dengue associated encephalopathy should be considered as differential diagnosis in patients having fever, thrombocytopenia and recent onset neurological deterioration or in patients having acute encephalitis/encephalopathy of unknown cause.

MRI is useful aid in diagnosis and GCS on admission is important prognostic factor. 


\section{3}

\section{Episodic Headache in Children: The Association of Headache with Epileptiform Discharges in the Electroencephalography}

Abdul-Latif A., Abdul-Latif M.

LaTrobe Neurology Centre, Welfare Hospital, Iraq

Background: Recurrent episodic headache in children is still a challenging issue in neurology. The accurate diagnosis is essential to differentiate between different types of headache particularly migraine that affect the type of management.

Methods: Thirty two children, aged between $6-14$ years old, have been included in this study. They were all presented with headache that occurs in recurrent episodes lasting between 5 minutes to 2 hours, once -4 times a month. 23 (72\%) of them reported post headache fatigue, confusion and / or increase desire to sleep.

Medical history was insignificant for all included patients. Clinical examination revealed no neurological deficit. The patients were sent for routine laboratory tests (Biochemistry and hematology), Brain MRI and EEG tests.

Results: Laboratory tests and Brain MRI were normal. EEG in all of the patients revealed epileptiform discharges of sharp wave, spike, or sharp and slow wave complexes in non-paroxysmal bursts. These abnormal EEG changes were seen bilaterally in an independent pattern.

Carbamazepine 200-400mg controlled release tablet was used in a single daily dosage for 6 months. All the patients showed satisfactory improvements reported as a decrease in the frequency of the attacks in the first 1-2 months then the disappearance of the headache while using the medications.

Conclusion(s): Episodic headache in children associated with abnormal EEG changes could be treated with anti-epileptic medication (carbamazepine).

The pathophysiology of this headache may be related to a type of vascular disorder that cause temporary reduction of cerebral blood flow causing excitation of cerebral cortex. This excitation is the cause of abnormal electrical activity of the brain reflected by the EEG.

\section{9}

\section{Spectral Signatures of Time Averaged EEG Source Density During Brain Disease in Comparison to a Norm Database of $\mathbf{3 0 0}$ Healthy Subjects}

Dimpfel W.

Justus-Liebig-University Giessen, Germany

Background: Conventional recording of the electroencephalogram (EEG) results in a very complicated depiction of potential differences whose interpretation takes a lot of skill and also is very time consuming. In addition, this subjective evaluation is not able to average data over time. But computer-aided quantitative frequency analysis based on Fast Fourier Transformation provides spectral power values within specially defined frequency ranges, which can be averaged during longer time of recording. This objective parametrisation now allows for individual comparisons. In order to uncover deviations from normality a norm database was needed.

Methods: Source density of the EEG spectra is calculated using a Laplacian algorithm. In order to use the information of the quantitative EEG for diagnostic purposes, a total of 300 healthy volunteers of both sexes aged 18 to 80 participating in our clinical studies served to construct a "norm-data-base". Individual data were averaged over a time period of 5 minutes. Distribution of 102 variables (17 electrode positions times 6 frequency ranges) serves for comparison of single patients to this database. According to the distribution function, an error probability for each single value was calculated. This value was called an "aberration index". For mapping of the EEG frequencies, power values were coded into spectral colours followed by additive colour mixture according to the RGB mode (like in TV). The same type of colour code was used for depiction of the original data and for statistical deviation from normality.

Results: With this index an objective measure for the occurrence of a functional disturbance of the brain has been developed, which not only is used as a diagnostic aid, but also serves for control for therapeutic success, as was shown recently in 30 patients suffering from Parkinson's disease after 4 months of treatment with rasagiline. Cumulative aberration indices decreased from 4.92 to 3.45. Brain regions involved decreased from 2.17 down to 1.4. Likewise, aberration from normality was shown in an epileptic patient with respect to theta power on electrode position T3 (statistical deviation from normality was 10000:1), which disappeared after successful treatment with valproic acid. No more seizures were observed for more than one year.

Conclusion(s): The definition of a statistically valid aberration index by using comparison of individual patient data to a norm database is not only useful for diagnostic purposes but also for the evaluation of the effect of medications.

\section{3 \\ Headache and Lower Back Pain as the Presenting Symptom of Idiopathic Meningeal Carcinomatosis}

OkS.

Korea University Ansan Hospital, South Korea

Background: Meningeal carcinomatosis is the malignant infiltration of the meninges from a distant solid tumor, most commonly adenocarcinoma of the breast or lung and melanoma. It is diagnosed mostly in patients with a known malignancy, and it is rare for a headache or lower back pain to be the first manifestation of idiopathic meningeal carcinomatosis (MC). We present here an unusual case of idiopathic MC marked by headache and lower back pain and treated with steroid pulse therapy.

Case report: A 39-year-old female came to hospital with headache for 2 weeks. Magnetic resonance imaging (MRI) of the brain, cervical and lumbar spine from 2 weeks ago showed left maxillary sinusitis, herniated nucleus pulposus at L5/S1, central spinal stenosis at $\mathrm{C} 5 / 6$ and questionable signal change at $\mathrm{C} 5 / 6$ 
level spinal cord. She got lumbar epidural steroid injection 2 weeks ago, after that injection lower back pain has subsided little bit, but headache was worsened as vomitting developed. We examined cerebrospinal fluid (CSF) and whole spine MRI with enhancement as soon as she came to emergency room, the CSF opening pressure was $28.5 \mathrm{~cm}$ and whole spine MRI showed leptomeningeal enhancement along the cerebellar folia/stem surface and film terminale. The meningeal biopsy revealed only severe fibrosis. There was no evidence of bacterial, fungal, tuberculous or neoplastic infiltration even though all the examinations including positron emission tomography (PET) of whole body. Idiopathic MC was diagnosed and steroid pulse therapy initiated. A week later, her headache subsided. But her level of consciousness started to deteriorate significantly over the next two weeks and other symptoms appeared: extraocular palsy, masseter palsy, ileus and acute renal failure. She died from cachexia 7 weeks after admission to our hospital. Idiopathic MC is a diagnosis of exclusion. The absence of underlying infective, neoplastic, or systemic autoimmune disease favors Idiopathic MC.

Discussion: The incidence of MC appears to be increasing, perhaps due to the longer survival of patients with malignancy. Nevertheless, $\mathrm{MC}$ is still considered infrequent, and its variable clinical manifestations make clinical diagnosis difficult, especially in patients with unknown malignancies. The clinical manifestations of MC are heterogeneous and symptoms are typically widespread, involving multiple levels of the central and peripheral nervous system. Symptoms like headache and lower back pain are very common, so idiopathic $\mathrm{MC}$ is difficult to be considered in the differential diagnosis. Median survival in untreated patients is 4-6 weeks, which may be extended up to 6 months with aggressive treatment in some cases. Both MRI scans and CSF evaluation are necessary diagnostic tools for idiopathic MC. Steroid pulse therapy is worth trying for treatment of idiopathic MC for a while.

\section{Session P3: Epidemiology}

\section{9 \\ A Common Link Between Three Sporadic Amyotrophic Lateral Sclerosis Cases in Annapolis, MD, USA}

Caller T. , Field N. ${ }^{\mathrm{a}}$, Banack S. ${ }^{\mathrm{b}}$, Cox P. ${ }^{\mathrm{b}}$, Metcalf J. ${ }^{\mathrm{b}}$, Stommel E. ${ }^{\mathrm{a}}$

${ }^{a}$ Dartmouth-Hitchcock Medical Center, USA; 'bnstitute for Ethnomedicine

Background: The majority of amyotrophic lateral sclerosis (ALS) cases occur sporadically, and spatial clustering of ALS has been described. A number of environmental triggers have been implicated, including beta-methylamino-L-alanine (BMAA), a cyanobacteria-produced neurotoxin linked to ALS on Guam. We report a cohort of three ALS patients diagnosed within 8 years of each other who lived for a minimum of 10 years on the same street in a suburb of Annapolis, Maryland, USA. Cyanobacteria blooms have been present in the Chesapeake Bay for decades, providing a potential for BMAA exposure through the food web in this region.

Methods: The diagnosis of ALS in all 3 cases was confirmed through their regional ALS Center. A questionnaire was completed by a surviving immediate family member of each patient, which ascertained lifelong dwelling history, exposure history and social history. The questionnaire also assessed recreational water use and potential exposure to cyanobacteria.

Results: One common exposure identified from the questionnaires was the regular consumption of freshly caught Chesapeake Bay blue crabs. Fresh Chesapeake Bay crabs from the same fish market where the three patients regularly bought their crabmeat were collected and tested for BMAA using well established methods. Two out of the three crabs tested were positive for BMAA.

Conclusion(s): The lifelong consumption of blue crab contaminated with BMAA may be a common risk factor for sporadic ALS in all three patients. Other factors may have also contributed to disease development.

\section{7 \\ Predicting Cognitive Impairment in Community- Dwelling Elderly Persons}

Jung-Wan $K^{\text {a }}$, Seung-In $K^{\text {b }}$

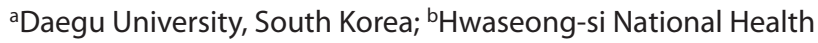
Center, South Korea

Background: Cognitive impairment is a major mental health problem affecting elderly persons. We examined the effects of social engagement, socioeconomic status, depression, age, sex, and level of education on cognitive functioning in communitydwelling elderly persons in Korea.

Methods: Between November 2009 and January 2011, we examined 449 subjects aged 65-92 years old, living in Gunpo, Korea. All participants were volunteers recruited from a local senior citizen's center. Mini mental status examination (MMSE) and Geriatric Depression Scale-Short Form (GDSSF) were used to assess the elderly's mental status. A social engagement scale was constructed from the following indicators: presence of a spouse, weekly contact with two or more relatives or friends, and regular physical activities. Socioeconomic status was rated as high, medium, or low.

Results: The prevalence of cognitive impairment in elderly persons was $14.25 \%$. The rates for depressive symptoms were $12.92 \%$. In the multiple regression analysis, frequency of contact with a relatives or friends, symptoms of depression, age, and level of education had an accountability of $26.3 \%$ to cognitive function. We found that poorer performance in attention \& calculation, delayed recall, and visuo-spatial construction are important characteristics of elderly at geriatric risk for cognitive decline.

Conclusion(s): In this research, vigorous social activity and symptoms of depression was the most powerful predictor of cognitive decline. Furthermore, older age and lower education level were significantly related to cognitive impairment among Korea subjects. Findings of this study may be useful in understanding 
the mental status of community-dwelling elderly persons and developing more specific public health services.

26

\section{Multiple Sclerosis Mortaliy and Dietary Factors in Spain: An Ecological Study}

Lauer K.

Germany

Background: Beside EBV infection, vitamin D deficiency and smoking, diet is also considered of importance in the aetiology of MS. An ecological correlation with the mortality or prevalence of multiple sclerosis (MS) was shown in the majority of regions with measures of production and/or consumption of smoked meat, and this association was confirmed in case-control studies. Food data from Spain were now tested for an ecological correlation.

Methods: The indirectly age-adjusted and sex-specific MS mortality (MS-M) in 47 provinces of Spain was published by Llorca et al. (Neuroepidemiology 2005;24:135). Food consumption originated from a household survey in 1964-1965, thus reflecting an earlier risk period. Spearman's rank correlation was applied. $\mathrm{P}</=0.05$ was considered as significant, and $0.05<\mathrm{p}<$ / $=0.10$ as borderline significant.

Results: In females, the following foods were correlated with the MS-M: potatoes $(r=0.292 ; \mathrm{p}=0.047)$; pork $(\mathrm{r}=0.298 ; \mathrm{p}=0.042)$; processed meat $(\mathrm{r}=0.270 ; \mathrm{p}=0.066)$; total meat $(\mathrm{r}=0.335 ; \mathrm{p}=$ $0.021)$; wine $(\mathrm{r}=0.337 ; \mathrm{p}=0.022)$; and other alcoholic beverages than wine, beer and liquors $(r=0.246 ; p=0.096)$. Vegetables and malt coffee were inversely related. In males, the MS-M was associated with: processed meat $(\mathrm{r}=0.269 ; \mathrm{p}=0.068)$; total meat $(\mathrm{r}=0.255$; $\mathrm{p}=0.084)$; tinned fish $(\mathrm{r}=0.283 ; \mathrm{p}=0.054)$; and wine $(\mathrm{r}=0.370 ; \mathrm{p}=$ 0.011 ). Cheese, sugar, malt coffee and tobacco were inversely related. When the interaction term "processed meat * firewood production by population," was tested, the correlation increased considerably (females: $\mathrm{r}=0.378, \mathrm{p}=0.009$; males: $\mathrm{r}=0.523, \mathrm{p}=0.0002$ ); no such interaction was evident with total meat consumption.

Conclusion(s): Processed meat consumtion was correlating with MS in Spain, as in the vast majority of other regions.

\section{5}

Clinical, Epidemiological, Demographical Study of Multiple Sclerosis Patients at the Department of Neurology / MS, Hospital de São Marcos de Braga, Portugal

Figueiredo J. ${ }^{\mathrm{a}}$, Pereira P. ${ }^{\mathrm{b}}$, Cerqueira J. ${ }^{\mathrm{b}}$

${ }^{a}$ Hospital Privado de Braga, Portugal; bUniversidade do Minho, Portugal

Background: Multiple Sclerosis (MS) is a chronic progressive inflammatory disease, demyelinating and degenerative, affecting the Central Nervous System (CNS). The importance of the disease in the current context is undeniable considering that it corresponds to the second cause of neurological disability in young adults in developed countries.

Our main goal is to characterize the MS population in the Hospital de São Marcos de Braga, that serves a population of 564.333 residents in the District of Braga, North of Portugal, with respect to prevalence, incidence and survival.

Methods: We considered the epidemiological measurements according to age, gender, and their diagnosis of MS. We will also present some preliminary results from the analysis of the geographical distribution of the disease and its possible relation with, explanatory variables as region of residence.

Results: In the present MS study, we found an estimated disease prevalence of 41, 6 per 100.000 inhabitants. In addition, for all MS patients followed at MS Outpatient Clinic, we report patient demographic characteristics (sex, age, age at diagnosis, treatment, spatial distribution of place of residence) and clinical outcome: type of the disease, treatment, clinical severity score (EDSS rating scale), year and month of diagnosis.

Conclusion(s): Our main conclusions were obtained comparing the results of this study with the ones from the other MS studies in Portugal. In this study we estimated a prevalence of 41, $6 / 100.000$.

For future work we intend to collect more cases in hospital and private clinics to get better estimates for prevalence and other epidemiological measurements in this region of Portugal.

\section{6}

\section{Neurofibromatosis Type 1 and Malignant Peripheral Nerve Sheath Tumour in Brazil}

Vasconcelos R. ${ }^{\mathrm{a}}$, Maia P. ${ }^{\mathrm{b}}$, Papaiz-Alvarenga R.M. ${ }^{\mathrm{a}}$, Rodrigues Santos C.E. ${ }^{\mathrm{b}}$

aUnirio, Brazil; ' Inca, Brazil

Background: The neurofibromatosis type 1 (NF1) is the most important syndrome related to cancer and the malignant peripheral nerve sheath tumour (MPNST) is its more frequently malignant complication. Both conditions are described over all the population of the world, but their numbers in brazilian population remain unknown.

Methods: To know the frequency of NF1 and MPNST in brazilian patients with soft tissue sarcomas (STS) we did a revision of data records of patients during a period of consective 10 years in a cancer treatment center.Descriptive statistical analysis was compound by percentage and mean.

Results: We found 447 patients with STS with next histopathological types: malignant fibrous histiocytoma -81 cases $(18.1 \%)$, Liposarcoma - 81 (18.1\%), Synovial sarcoma - 56 (12.5\%), Spindle cell sarc. - 49 (11.0\%), Fibrosarcoma - 51 (11.4\%), Leiomyosarcoma - 26 (5.9\%), Desmoid-type fibromatoses - 20 (4.5\%), Rhabdomyosarcoma - 12 (2.7\%), MPNST - 34 (7.6\%), Epitheliod sarc. -11 (2.5\%), Dermatofibrosarcoma protuberans 4 cases $(0.9 \%)$, Alveolar sarcoma - 6 (1.3\%), Clear cell sarcoma - 5 (1.1\%), Chondrosarcoma - 2 (0.4\%), Pnet - 3 (0.7\%), Sclerosing epithelioid fibrosarcoma - $1(0.2 \%)$, Fibromyxoide sarc. - 1 
(0.2\%), Hemangiopericytoma - $1(0.2 \%)$, Myxoinflammatory sarc. - $1(0.2 \%)$, Osteosarcoma - $1(0.2 \%)$, Giant celltumour - 1 $(0.2 \%)$.

The frequency of NF1 in patients with STS was 3, 8\% (17 cases).

MPNST was 7, 9\% (34).

MPNST with NF1 was 17 cases (50\%), 11 male and 6 female; average age 38 years (12-63); 5 white, 6 black and 6 mixed.

MPNST without NF1: 17 cases, 5 male and 12 female; mean age 58 years (17-92); 11 white, 2 black and 4 mixed.

The histological type of STS in all patients with NF1 was MPNST.

Conclusion(s): In patients with STS the NF1 was present in 3, $8 \%$, all these cases with MPNST. MPNST was 7, $6 \%$ of all STS, $50 \%$ had NF1.

\section{9 \\ Circulating IL-6 and the Severity of Dilated Virchow- Robin Spaces in the Elderly \\ Satizabal C. ', Zhu Y.-C. ${ }^{\text {, }}$ Dufouil C. ${ }^{\text {, }}$ Tzourio C. ${ }^{c}$ \\ aINSERM U708, Université Pierre et Marie Curie, Bordeaux, France; beking Union Medical College Hospital, Bejing, China; 'Université Victor Segalen Bordeaux 2, France}

Background: Virchow-Robin Spaces are perivascular compartments surrounding the small penetrating cerebral vessels. Recent studies suggest dilated Virchow-Robin Spaces (dVRS) could be a manifestation of cerebral small-vessel disease, but little is known about their risk factors. As inflammation has been associated with other brain MRI findings, we investigated whether interleukin-6 (IL-6) was associated with the severity of dVRS in elders.

Methods: Our sample included 1820 participants from the 3C-Dijon Study aged 65 to 80 years. IL- 6 concentrations were measured from fasting blood samples. dVRS were assessed in basal ganglia and white matter using high-resolution MRI and then rated on a severity scale. The association between elevated IL-6 levels and the severity of dVRS in basal ganglia or white matter were modeled with multinomial logistic regression adjusting for age, gender, hypertension and total intracranial volume.

Results: We found that participants with elevated IL-6 levels, relative to those with lower IL-6 levels, were more likely to have 2nd degree dVRS (OR $=1.23$; 95\% CI 1.0-1.5) and even more likely to have 3 rd degree dVRS and above (OR = $1.38(1.0-2.9)$ than 1st degree dVRS in basal ganglia. No associations were found between IL-6 levels and dVRS in white matter.

Conclusion(s): These results suggest that the presence of this MRI finding may be accompanied by an inflammatory process in aging population and dissimilar mechanisms underlying the enlargement of perivascular spaces in basal ganglia and white matter. This association might provide additional data supporting the contribution of inflammation to cerebral small-vessel disease.

\section{1 \\ Cognitive Tests Reliability in a Brazilian Multicentric Cohort Study of Adults}

Passos V., Alves B.J., Giatti L., Barreto S.

Faculty of Medicine, Universidade Federal de Minas Gerais, Brazil

Background: A test-retest reliability study was conducted in order to assure quality of cognitive function evaluation in the Brazilian Longitudinal Study of Adult Health (ELSA-BRASIL), a multicentric cohort study that is being carried out with the aim of investigating risk factors for development and progression of chronic diseases.

Methods: The same battery of cognitive tests, in different order of appearance, were applied by the same interviewer, with an approximate 20-day interval, among 160 adults (age 35-74 years). The Intraclass Correlation Coefficient (ICC) was used to measure the reliability of ratings, and a Bland-Altman plot was included to examine the patterns of ratings disagreement.

Results: On retest, it was observed a significantly increase of the scores of all cognitive tests. ICC values for each cognitive test were as following: CERAD's Word List Learning Test (0.56), Word Recall (0.50), Word Recognition (0.35), Phonemic Verbal Fluency Test (0.61), Semantic Verbal Fluency Test (0.53) and Trail Test B (0.91). The Bland-Altman plot showed better correlation of executive function tests (Verbal Fluency and Trail Test) than memory tests.

Conclusions: Overall increase of cognitive scores in retest may be related to learning effect, and suggests that retest should be repeated after a longer period than the interval used in this study Although largely utilized in the country, this first Brazilian reliability study 1 shows, in a sample of higher schooling individuals than the general population, that the reliability was only moderate for memory tests and, the measurement of executive function is more reliable than memory measurements.

62

Prevalence of Dementia and Dementing Diseases in the Old-Old Population in Northern Japan: The Osaki and Kurihara (Miyagi Prefecture) Projects

Meguro K.

Tohoku University Graduate School of Medicine, Japan

Background: There have been no reports on the prevalence of dementia and dementing diseases among the old-old people in Japan. Vascular contributions to Alzheimer's disease (AD) with cerebrovascular diseases (CVD), vascular dementia (VaD) have not been clarified.

Methods: We targeted the selected old-old population aged $75+$ years by block randomization in Osaki $(\mathrm{n}=593)$ and Kurihara $(\mathrm{n}=1,252)$ (total old-old population, 17, 553 in Osaki and 14, 579 in Kurihara), northern Japan. Totally, 754 people agreed to participate $(4.09 \%)$ : the prevalence of dementia and dementing dis-

Neuroepidemiology 2012;39:177-283

Poster Presentations 
eases were analyzed by intensive evaluation including MRI. Locations of CVD were also assessed for the patients with $\mathrm{AD}$ with CVD and VaD.

Results: Eighty nine (11.8\%) people were diagnosed with dementia (DSM-IV). The most common cause was AD with CVD, followed by AD (NINCDS-ADRDA) and VaD (NINDS-AIREN). The AD with CVD group had non-"strategic" CVDs, whereas the$\mathrm{VaD}$ group had at least cortical CVDs or subcortical "strategic" CVDs including the thalamus or caudate head.

Conclusion(s): The prevalence of dementia among the old-old population was higher than that reported previously. AD with CVD was the most common cause, coincided with the previous findings of $65+$ years; however, the ratio of $\mathrm{VaD}$ was greater. Our data showed an importance of subcortical lesion for causing cognitive deterioration.

\section{7 \\ The Vanishing Deleterious Effect of Weekends and Bank Holidays on Stroke Mortality: A 26-Year Population-Based Study}

Béjot Y., Aboa-Eboulé C., Jacquin A., Durier J., Osseby G.V., Giroud M.

Registre dijonnais des AVC, EA 4184, IFR 100 Santé-STIC, France

Background: Suffering a stroke during the weekend may be associated with a poorer prognosis because of a lower quality of stroke care. We evaluated the effect of weekends/bank holidays on 30-day mortality and its temporal trends.

Methods: All cases of stroke and transient ischemic attack (TIA) from 1985 to 2010 were identified from the populationbased Stroke Registry of Dijon, France. Clinical information and day of onset (weekdays versus weekends or bank holidays) were recorded. Cox regression models were used to evaluate the associations between day of onset and 30-day all-cause mortality.

Results: Among the 5864 recorded patients with stroke/TIA, 1465 (25\%) had their event during weekends or bank holidays.

Thirty-day mortality in patients with stroke/TIA during weekends/bank holidays was greater than in those with events during weekdays (15.2\% versus $12.1 \%$, unadjusted HR 1.27 ; $95 \% \mathrm{CI}$ : $1.08-1.48, \mathrm{p}<0.01)$. In multivariate analysis, weekend/bank holiday onset was a predictor of 30-day mortality (HR 1.19; 95\% CI: $1.02-1.39, \mathrm{p}=0.03)$, especially in patients with decreased consciousness 1.32 (95\% CI: 1.09-1.60, p < 0.01). A decreasing trend in the excess mortality was noted over time (HR 1.31; 95\% CI: 1.04-1.69 for $1985-1993$, HR 1.21; 95\% CI: $0.94-1.56$ for $1994-$ 2003, and HR 1.03; 95\% CI: 0.72-1.48 for 2004-2010).

Conclusion: The deleterious effect of weekends and bank holidays on early stroke mortality did exist but has declined over time, probably due to major changes in stroke care organisation in our community. Our findings lend support to initiatives to improve care delivery and outcomes in patients with stroke.

\section{0}

\section{Short-Term and Long-Term Stroke Survival: The Belgrade Prognostic Study}

\section{Pekmezovic T. ${ }^{\text {, }}$ Sanja M. ${ }^{\text {, }}$, Beslac-Bumbasirevic L.,} Kisic-Tepavcevic D. ${ }^{\text {a }}$

aFaculty of Medicine, University of Belgrade, Yugoslavia;

bepartment of Neurology, Clinical Center Dr Dragisa Misovic

Background: The aims of this study are to determine the 28-day and the 1-year survival probabilities after first-ever ischemic stroke and to identify the baseline predictors of mortality.

Methods: We prospectively and consecutively collected data on 300 patients with first-ever acute ischemic stroke admitted to two main neurological institutions for cerebrovascular diseases in Belgrade, during March 2008. The Kaplan-Meier method was used to estimate the cumulative survival probability of 28 days and one year. We defined stroke onset as zero time and death as end-point. The predictive value of different variables was assessed by Cox proportional hazard regression model.

Results: The cumulative 28-day and 1-year survival probability of ischemic stroke patients in the cohort were $81.0 \%$ and 1 -year $78.3 \%$, respectively. The highest cumulative 28 -day survival probability was observed in patients with small artery occlusion (94.1\%), and the lowest one in those with cardioemolism (65.4\%) $(p=0.001)$. The multivariate predictive model revealed that hypertension ( $p=0.017)$, NIHSS score $(p=0.001)$, and in-hospital medical complications $(\mathrm{p}=0.029)$ were significant unfavorable independent outcome predictors, while early physical therapy ( $\mathrm{p}$ $=0.001$ ) was significant favorable prognostic factor for 28-day mortality in our first-ever ischemic stroke patients. Multivariate Cox regression analysis showed that age $(\mathrm{p}=0.001)$, NIHSS score $(\mathrm{p}=0.001)$ and in-hospital complications $(\mathrm{p}=0.008)$ remained significant predictors of 1-year mortality.

Conclusion(s): The findings support the need for optimal control of vascular risk factors and careful treatment of atherosclerotic vascular disease as well as appropriate prevention and management of in-hospital complications, in order to improve short-term and long-term prognoses after an ischemic stroke.

\section{3}

\section{Effects of Clopidogrel and Aspirin versus Aspirin Alone on Hematological Parameters: Retrospective Database Study in a Japanese Population}

Takahashi Y., Nishida Y., Asai S.

Nihon University School of Medicine, Japan

Background: Clopidogrel and aspirin are antiplatelet agents that are recommended to reduce the risk of recurrent stroke and other cardiovascular events. Dual antiplatelet therapy with clopidogrel and aspirin has been shown to increase the risk of hemorrhage, but the effects of the drugs on laboratory parameters have not been studied in clinical settings. This poster presents a retrospective observational, cohort study to compare the effects of 
combination therapy with clopidogrel plus aspirin and aspirin monotherapy on hematological parameters.

Methods: We used study data from the Clinical Data Warehouse of Nihon University School of Medicine obtained between Nov 1, 2004 and May 31, 2011, to identify cohorts of new users $(n=130)$ of clopidogrel (75 mg per day) plus aspirin (100 $\mathrm{mg}$ per day) and propensity-score matched new users of aspirin alone $(n=130)$. We used a linear regression model to compare laboratory parameters including hemoglobin level, hematocrit, and white blood cell (WBC), red blood cell (RBC) and platelet counts up to 2 months after the start of study drug administration.

Results: After propensity-score matching, there were no significant differences for all characteristics between clopidogrel plus aspirin users and aspirin alone users. The reductions of WBC and $\mathrm{RBC}$ counts, hemoglobin level and hematocrit in clopidogrelplus aspirin users were significantly greater than those in aspirin alone users.

Conclusion(s): Our study suggested that hematological adverse effects may be greater with combination therapy of clopidogrel plus aspirin than with aspirin monotherapy.

\section{5 \\ Visit-To-Visit Variability in Systolic Blood Pressure and White Matter Lesions: A Community-Dwelling Elderly Cohort, the $3 C$ Study}

Soumare A. ${ }^{a}$, Alperovitch Y. ${ }^{\mathrm{a}}$, Zhu Y.-C. ${ }^{\mathrm{b}}$, Mazoyer B. ${ }^{\mathrm{c}}$, Dufouil C. ${ }^{\mathrm{a}}$, Tzourio C. ${ }^{\text {" }}$

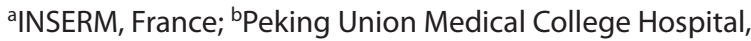
Beijing, China; 'CNRS-CEA; ${ }^{d}$ Université Victor Segalen

Bordeaux-2, France

Background: White matter hyperintensities' (WMH) etiology remains unclear. Variability of blood pressure might be a contributing factor via its impact on cerebral blood flow. We studied the relationship between visit-to-visit variability in systolic blood pressure (SBP) and $\mathrm{WMH}$ at baseline and over time in community-dwelling elderly from the Dijon (France) center of the ThreeCity cohort study.

Methods: 1,300 participants aged 80 years or younger who had BP measured by electronic devices during the 3 study visits at 2 -year intervals, and who underwent a cerebral magnetic resonance imaging (baseline and 4-year examination). Volumes of WMH (total, periventricular, deep) were estimated using a fully automatic procedure. Linear regression models were used to assess the association between SBP variability (Standard Deviation/ mean SBP) and WMH at baseline and over time.

Results: Increased SBP variability was associated with higher baseline total $(\Omega[\mathrm{SE}]=0.023[0.008], \mathrm{P}=0.003)$ and periventricular $\mathrm{WMH}(\Omega[\mathrm{SE}]=0.026[0.009], \mathrm{P}=0.003)$ volumes. These associations were independent of potential confounders including age and gender. Moreover they were not modified by mean SBP level across visits ( $\mathrm{P}$ for interaction $>0.44$ ). Those with higher SBP variability had higher increase of WMH (total, periventricular) over time, although this increase was not statistically significant.
Conclusion(s): Visit-to-visit variability in SBP correlated with $\mathrm{WMH}$ volumes, in particular in the periventricular region. These findings reinforce the hypothesis of a hypoxic component of $\mathrm{WMH}$ and suggest that limiting $\mathrm{BP}$ variability might help to reduce the burden of $\mathrm{WMH}$.

\section{7 \\ Prior Transient Ischemic Attack (TIA) and Early Dementia After Subsequent Ischemic Stroke}

Pupillo E. ${ }^{a}$, Beghi E. ', Chiò A. ${ }^{\mathrm{b}}$, Logroscino G. ${ }^{\mathrm{b}}$, Preux P.M. ${ }^{\mathrm{c}}$, Mitchell D. ${ }^{\mathrm{d}}$, Hardiman O. ${ }^{\mathrm{e}}$, Messina P. ${ }^{\mathrm{f}}$, Giussani G. ${ }^{\mathrm{f}}$

aMario Negri Institute for the Pharmacological Research, Italy; bAOU “LeMolinette"-s.Giovanni Battista, Italy; 'University of

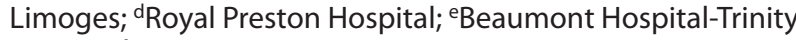
College; ' Mario Negri Institute for Pharmacological Research, Italy

Background: Amyotrophic lateral sclerosis (ALS) is a rare and severe clinical condition of unknown etiology. Environmental factors have been implicated, including exaggerated physical activity, but no single factor has been consistently associated with the disease. The study purpose was to assess whether physical activity is a risk factor for ALS in a large representative population.

Methods: From February 2008 to April 2012, 461 patients with ALS (El-Escorial Classification) from European populationbased registries (France, Ireland, Italy, United Kingdom) and 922 population controls (matched for age, sex, and residency) were enrolled. Upon direct interview, data were collected on occupation and history of sport and leisure activities, physical activity, and accidental injuries. Physical activity was coded as none, jobrelated and sport-related, the latter stratified into amateur, organized or professional. Risks were calculated using conditional logistic regression model (adjusting for age, country, trauma and job-related physical activity) and expressed as odds ratios (OR) with $95 \%$ confidence intervals (CI).

Results: The sample had a slight male predominance $(56.0 \%$ vs. $44.0 \%)$ and a median (range) age of 66 years (28-89)(ALS) and 67 years (30-93)(controls). Job-related physical activity was reported by $122(26.5 \%)$ and $335(36.5 \%)$ cases and controls. Numbers for sport-related amateur, organized and professional activity were $172(37.6 \%)$ and $363(39.6 \%), 65(14.2 \%)$ and 211 (23.0\%) and $12(2.6 \%)$ and 17 (1.9\%). In the logistic regression model, amateur and organized sport activity were inversely correlated to ALS (OR 0.62, 95\%CI 0.46-0.84 and, respectively, 0.36, $95 \%$ CI $0.24-0.55)$ while professional activity was unrelated (OR 0.97, 95\%CI 0.41-2.32).

Conclusion(s): Sport-related physical activity may be protective for ALS if non-professional. 
80

\section{Epidemiology of Syringomyelia and Chiari Malformations: Role of Interpopulation Studies of the Phenotype of Posterior Cranial Fossa}

Zabbarova A. ${ }^{\mathrm{a}}$, Bogdanov E. ${ }^{\mathrm{a}}$, Odin P. ${ }^{\mathrm{b}}$, Kyoshima K. ${ }^{\mathrm{c}}$, Pfleiderer S. ${ }^{\mathrm{d}}$, Honig $\mathrm{H}^{\mathrm{b}}{ }^{\mathrm{b}}$, Mikhailov l. ${ }^{\mathrm{a}}$

${ }^{a}$ Kazan State Medical University, Russia; ${ }^{b}$ Department of Neurology, Klinikum-Bremerhaven, Germany; 'Shinshu University School of Medicine, Nagano, Japan; ${ }^{d}$ KlinikumBremerhaven, Germany

Background: Detection of the association Chiari-like symptoms and syringomyelia with phenomenon of "overcrowded" posterior cranial fossa (PCF) has caused in recent years interest to PCF craniometry. The identification of ethno-geographical differences in prevalence of Chiari-associated syringomyelia has substantiated the prospects of interpopulation standardized studies of phenotype of PCF.

Methods: We performed a pilot study of 50 craniometric parameters of "normal" PCF patients from three geographic regions with different prevalence of syringomyelia and Chiari malformation type 1: regions with low prevalence - group $1(\mathrm{n}=$ 61; Lower Saxony, Germany) and group $2(\mathrm{n}=60$, Nagano Prefecture, Japan), a region with a high prevalence - group $3(\mathrm{n}=$ 79, Tatarstan, Russia). To study were selected the MRI images (1 Tesla) of adult patients without focal changes in the brain, hydrocephalus, cerebellar tonsillar ectopia, tight cisterna magna, syringomyelia, basilar impression.

Results: For every region, PCF in men in comparison with women were characterized by large size (the 17 indicators) and did not differ in its shape. The mean values of six parameters PCF in women and of four - in men were not significantly different between all groups. We found the relationship between geographical factor and PCF phenotype: in group 1 - long PCF with largest volume, minimum length of foramen magnum and minimum cerebrospinal kinking; in group 2 - high and narrow PCF, with greatest width of cerebrospinal fluid spaces; in group 3 - broad and low PCF, with a flattened skull base, smallest width of cerebrospinal fluid spaces and greatest width of upper cervical spinal canal. The greatest overcrowdedness of the "normal" PCF has been featured in group 3 of patients living in region with highest prevalence of syringomyelia and Chiari malformation.

Conclusion(s): Continue of interpopulation studies of genetic and environmental components of geographical factor can be useful for diagnosis and prevention of clinical syndromes of PCF.

\section{3}

\section{The Distribution of Stroke Among the Japanese Is} Quite Different from That of the European Countries

\author{
Izumi M. a, Suzuki K. ${ }^{\text {b }}$
}

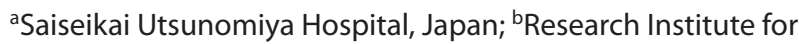
Brain and Blood Vessels, Akita, Japan

Background: Stroke has been a leading cause of mortality of disability all over the world. Numerous attempts have been made by scholars to demonstrate the characteristics of strokes, such as distribution, risk factors, mortalities and so on. Although a large number of studies have been made on accumulating evidence among Caucasian, little is known about the epidemiological evidence among the Japanese. The purpose of this study is to reveal the epidemiological differentiation of stroke among the races.

Methods: We have evaluated the total of 36305 incidence of strokes (ischemic, hemorrhage and subarachnoid hemorrhage) among Akita Stroke Registry from 1995 to 2004. Akita Stroke Registry consisted of the basis of informed consent from all the patients with all stroke events and stroke related death that required immediate hospitalization in all emergency medical facilities of Akita Prefecture. The incidence of strokes is adjusted with age, gender and distribution of population among European countries.

Results: The incidences of intra-cranial hemorrhages in each countries were 28\% (Japan), 11\% (France, Australia and Italy) and $10 \%$ (Sweden). The incidences of subarachnoid hemorrhage were 14\% (Japan), 1\% (France) and 5\% (Australia). The incidences of ischemic stoke were 58\% (Japan), 79\% (Italy) and 82\% (Denmark). The characteristics of stroke distribution in Japan were quite different among those of European countries. In other words, the Japanese have the higher tendency to have hemorrhagic stroke and the lower tendency to have ischemic stroke than the Europeans.

Conclusion(s): Compared with European countries, the incidence and distribution of strokes were significantly different. We must have to focus on the differences among races to develop strategies for each types of stroke.

\section{7 \\ Age and Sex Specific Mortality in Amyotrophic Lateral Sclerosis (ALS)}

Messina M., Pupillo E., Millul A.

Mario Negri Institute for Pharmacological Research, Italy

Background: Information on excess mortality of ALS by sex and age is limited. The aim of this study was to assess excess mortality of ALS using the standardized mortality ratio (SMR) by sex, age and symptom onset.

Methods: All consecutive patients from a population-based registry diagnosed during the years 1998-2002 in nine provinces of Lombardy, Northern Italy (population 4,947,554) were followed until death or to April 2012, whichever came first. Observed 
deaths occurred within 3 and 5 years after ALS diagnosis were compared to the expected deaths obtained from the statistical database (ISTAT) for the Lombardy region using SMRs.

Results: Included were 517 patients. Fifteen cases were later excluded because they were duplicate (10), the diagnosis was outside the study period (3) or were not resident in Lombardy (2). After diagnosis confirmation (still ongoing) other 38 patients were excluded because found not to have ALS (24) or unavailable (14). Therefore, the analysis was conducted in 464 patients, 199 (42.9\%) females, 296 (64.8\%) spinal-onset, 209 (45.0\%) with definite ALS at inclusion. Mortality within 5 years after diagnosis was significantly increased for the youngest age $(<54 \mathrm{y})$ with differences in sex (women: SMRs (95\%CIs): $20-44 y=51.5(0.0,123.0)$, $45-54 \mathrm{y}=47.8(18.2,77.4)$; men: $20-44 \mathrm{y}=20.4(7.1,33.7), 45-54 \mathrm{y}$ $=24.3(13.7,35.0))$. Spinal-onset SMRs ranged from 31.1 (17.8, $44.5)$ to $0.9(0.0,2.0)$, bulbar-onset from $43.4(0.9,86.0)$ to $0.9(0.0$, 1.7). Unremarkable SMRs were above 85 years old both for women and men $(0.8$ and 1.0$)$, and at $75-84 y$ in men $(1.1,2.2)$. Results obtained within 3 years after diagnosis were similar with lower risks.

Conclusion(s): The odds of death in very elderly people with ALS is no different from those expected in the general population.

91

\section{Care Management for ALS Patients}

Doi Y. ${ }^{\mathrm{a}}$, Nakano I. ${ }^{\mathrm{b}}$, Atsuta N. ${ }^{\mathrm{c}}$, Morita M. ${ }^{\mathrm{b}}$, Sobue G. ${ }^{\mathrm{c}}$

${ }^{a}$ National Institute of Public Health, Japan; ${ }^{b} J i c h i$ Medical University School of Medicine; 'Nagoya University Graduate School of Medicine, Japan

Background: Amyotrophic lateral sclerosis (ALS) is a progressive and fatal neurodegenerative disease with a median survival of 3-5 years after diagnosis. With advances in medical and communication technology, patients can live longer than ever before. Thus, as part of the program designated "Research on CNS Degenerative Diseases in Japan", we conducted an investigation of nutritional and respiratory care for ALS patients.

Methods: We examined 7,461 ALS patients registered to the national database in Japan from April 2009 to March 2010 (1,795 new and 5, 666 renewed patients). We performed descriptive analyses of the frequency and duration of care (tube feeding, noninvasive positive pressure ventilation [NIPPV] and tracheostomy positive pressure ventilation [TPPV]) according to living conditions, and the duration from disease onset to the introduction of care according to initial symptoms.

Results: Patients were 4,305 men and 3,156 women, and 66.9 $( \pm 11.0)$ years old in 2009 . Overall, $32.3 \%, 7.5 \%$ and $25.1 \%$ of ALS patients were managed with tube feeding, NIPPV and TPPV, respectively. The corresponding durations of such cares (median) were 36.1, 21.6 and 51.3 months. Patients in hospitals and nursing-care facilities were more likely to use the cares than those at work, school, performing household work or receiving home care. The corresponding durations from disease onset to the introduction of those cares were 11.5, 11.1 and 5.5 months. Onset with respiratory distress caused increased-ratio and rapid introduction of NIPPV and TPPV.

Conclusion: Special attention should be paid to ALS patients with respiratory distress at onset to support them properly.

\section{8 \\ Comparison of Clinical Features in Patients with Pediatric and Young Adult Onset Multiple Sclerosis}

Ragonese P., Mazzola M.A., Lo Re V., Realmuto S., Lo Re M., Vazzoler G., D'Amelio M., Aridon P., Salemi G., Savettieri G.

University of Palermo, Italy

Background: Approximately, 5\% of Multiple sclerosis (MS) patients develop their first symptoms in childhood or adolescence but there is still lack of concordance about clinical features of these patients, because of the different definitions used to classify the condition. Objective. To investigate characteristics of early onset MS patients and to compare them with patients who experienced MS onset at later ages.

Methods: We included patients affected by MS who experienced their onset of symptoms until the age of 25. MS patient were then categorized in three groups according to the age at onset; group A: until 16 years old, group B: 17-20, and group C: $21-25$ years old. Characteristics of these groups were compared with respect to the following variables: sex ratio, disease onset (mono vs. polisymptomatic or ADEM), clinical presentation at onset of symptmos, paraclinicalcriteria like proportion of patients with positive oligoclonal bands (OCB), MRI, proportion of relapsing patients, frequency of relapses, disability at follow-up measured by EDSS.

Results: We included 526 MS patients (74 group A, 162 group B, 290 groupC). Sex ratio varied from 1.55:1 in the first group, to 2.24 and 1.99 in the second and third respectively. Only 5 individuals (1\%) had a progressive course since the onset (one in the younger group and two in each of the others). A poly-symptomatic onset occurred in 16.2 and $17.9 \%$ of group A and B, compared to the $11 \%$ of the patients in group $\mathrm{C}(\mathrm{p}=0.2$ and $\mathrm{p}=0.04$ respectively). We did not observed significant differences in the rates of patients positive for OCB in the three groups (85\% group A, $74.1 \%$ group $B, 77.1 \%$ group $C$ ).

Conclusion: This study confirm that individuals with a younger onset MS present characteristics different from patients with an older age at onset. 
99

\section{Anthropological and Epidemiological Study of Epilepsy in the Region of Tangier (Morocco)}

Bahbiti Y., Benazzouz B. ${ }^{\text {a }}$ Moutaouakil F. ${ }^{\text {, }}$, Ouichou A. , El Hessni A. ${ }^{a}$, Mesfioui $A{ }^{\text {a }}$

aLaboratory of Genetics, Neuroendocrinology and Biotechnology, Unit of Nervous and Endocrine Physiology, Department of Biology, Faculty of Sciences, University lbn Tofail, Kenitra, Morocco; ${ }^{b}$ Department of Neurology, Al Kortobi Hospital, Tangier, Morocco

Epilepsy is a chronic neurological disorder characterized by recurrent seizures.

Objective: determine the knowledge, attitudes and beliefs of the public towards epilepsy in Tangier region (Northern Morocco).

Methodology: Performed in the Neurology Department at the Hospital El Kortobi Tangier, via a standardized questionnaire about epilepsy, conducted on 180 participants (patients and family members).

Results: The study revealed that the disease is unknown in this region because $66.1 \%$ of respondents have not "read" or "heard", about a disease called "epilepsy", $60 \%$ did not know a person with epilepsy, yet $90 \%$ of respondents attended convulsions. Concerning the public's attitude towards epilepsy, the least we can say is that the rejection and marginalization watch with epilepsy: $70 \%$ do not allow their children to play with people who may suffer from convulsions. $80 \%$ of the participants not allow their children to marry sometimes with people making seizures. $50.6 \%$ of respondents believed that epileptics cannot exercise any craft. Worse, $40 \%$ of respondents believed that epilepsy is a form of mental retardation. $15.6 \%$ of people interviewed believe that the main cause of epilepsy is supernatural (jinn, witchcraft) and 20.6\% think it's caused by stress, $15.6 \%$ think it is related to the alcohol and drugs. As for treatment, $68.9 \%$ of our sample suggested to ask doctor advice to treat epilepsy, $46.7 \%$ using Fkih's help, $8.3 \%$ see a psychologist. $11.10 \%$ of people with epilepsy use the plant Ruta graveolens to treat epilepsy.

Conclusions: Epilepsy remains a misunderstood disease and still subject to wrong interpretation. Awareness campaigns should be conducted to clarify certain aspects of epilepsy.

\section{2 \\ Bacterial Brain Abscess Formation: Is It a Delayed Complication of Radiation Therapy?}

Chuang Jimmy M.-J.

Kaohsiung Chang Gung Memorial Hospital, Taiwan

Background: Until recently, post-radiotherapy brain abscess was considered rare, but it has become an increasingly important etiology in Taiwan. Discussions on the relationship between bacterial brain abscess and radiotherapy (RT) are rare in the literature. Our clinical study was conducted to analyze the role of RT in the pathogenesis of bacterial brain abscess, with the objective of improving therapeutic strategies for this potentially fatal infection of the central nervous system (CNS).

Methods: For our retrospective study, 146 patients with bacterial brain abscess were recruited. Ten patients with a history of RT before brain abscess formation were reviewed.

Results: Ten patients had a history of RT before bacterial brain abscess formation. Eight of these patients underwent RT treatment for nasopharyngeal carcinoma. One received RT for olfactory neuroblastoma, and another for nasal plasmacytoma. Statistically significant differences between the RT- and non-RT patients were found for radionecrosis, bone defect between the middle fossa/ sphenoid sinus, and the presence of nasal infection/otitis media. The mortality rate in post-irradiated patients was $30 \%$.

Conclusion(s): Both the radiation effects and the tumor effects contributed to the pathogenesis of bacterial brain abscess formation in post-irradiated patients. Post-irradiated patients with a skull base defect were prone to CNS infection.

For such patients, aggressive treatment for nasal infection or chronic otitis media may be required. Radiation-related temporal lobe necrosis was a predisposing factor. However, we do not support radionecrosis resection. Further study of the pathogenesis of bacterial brain abscess formation in post-irradiated patients is warranted.

\section{6 \\ Detection and Quantification of Human Herpesvirus 6 in Samples from Patients with AIDS and Progressive Multifocal Leukoencephalopathy}

Fink M., Canto C., Sumita L., Boas L., Vidal J., Penalva A., Pannuti C. Instituto de Medicina Tropical de São Paulo, Brazil

Background: Progressive multifocal leukoencephalopathy (PML) is a demyelinating disease of the central nervous system (CNS). PML usually occurs in immunocompromised patients and mainly in aids. There are few reports suggesting the association between JCV and HHV6 in PML and other demyelinating diseases.

The aim of this study was to investigate the presence and quantification of HHV6 DNA in samples of cerebrospinal fluid from patients with PML and other neurological diseases, by Sybr Green real time PCR.

Methods: Cerebrospinal fluid samples from patients with aids with suspected PML were tested for HHV6. DNA was extracted by QIAamp ${ }^{\circledast}$ DNA Mini Kit.Five microliters of DNA were analyzed for the presence of HHV6 by Sybr Green Real Time PCR $\left(\right.$ Qiagem $\left.^{\oplus}\right)$ with primers which codify the main viral capsid protein.

Results: Among the 82 patients investigated, HHV6 DNA was found in $4(4.87 \%)$. One of them had a PML diagnosis confirmed by clinical, neuroimaging and laboratorial findings. JCV DNA was not detected in the other three positive samples.

Conclusion(s): Our data show that, despite being a rare occurrence, the presence of HHV6 in cerebrospinal fluid samples from patients with aids and PML can be detected. 
107

\section{Herpes DNA Detection in Multiple Sclerosis}

Fink M., Sumita L., Olival G., Vidal J., Nali L., Romano C., Moraes L., Casseb J., Penalva A.

Instituto de Medicina Tropical de SãoPaulo, Brazil

Background: The etiology of MS is still unknown and is considered a multifactor disease. One theory suggests that MS is triggered by an infectious agent, andviruses are main candidates. Although many different viruses have been isolated from cerebrospinal fluid or tissue of the central nervous system, has not been possibleto establish the relationship of an agent, or a group of infectious agents with MS. Among viruses the main candidates are the human herpes virus. We evaluate the presence of herpesviruses DNA in the blood by Polymerase Chain Reaction (PCR) for in MS patients, with special attention to patients with immunosuppressant drugs.

Methods: The study included 93 patients from the outpatient of public hospitals of Sao Paulo. Blood samples were sent to the Virology laboratory of theInstituto de Medicina Tropical de São Paulo. DNA extraction was performed on whole blood, using the QIAmp DNA Mini Kit from Qiagen ${ }^{\circledR}$. A panel of herpesviruses ("panherpes") PCR test was performed. After amplification was carried out an enzymatic reaction to determine eight subtypes.

Results: We analyses 93 blood samples. Of these, $35.5 \%$ (33/93) were positive for a herpesvirus. The subtype's virus was identified: HHV-7 in $75.8 \%$ (25/33); EBV $12 \%$ (4/33) and HHV-6A in 3\% (1/33). In three patients $(9 \%)$ detected the presence of EBV DNA viruses and HHV-7 concurrently. In the 33 patients with herpesvirus DNA detected, 78.8\% (26/33) were not receiving immunosuppressant drugs, whereas 7 (21.2\%) were undergoing natalizumab treatment. In the study group, 14 patients (15\%) undergoing natalizumab treatment were not identified any herpesvirus DNA.

Conclusion(s): The preliminary results obtained so far showed a high prevalence of HHV-7 on blood samples in the study population.

\section{0}

Trends in Stroke Hospitalisation Rates and Case Fatality in Aragon (Spain) from 1998 to 2010

Giménez-Muñoz A. ${ }^{a}$, Ara Jose R. ${ }^{\mathrm{b}}$, Abad Diez J. ', Campello I. ${ }^{\mathrm{a}}$, Marta J. ${ }^{\mathrm{b}}$, López Gastón J. ${ }^{\mathrm{b}}$, Ara Jose R. ${ }^{\mathrm{b}}$

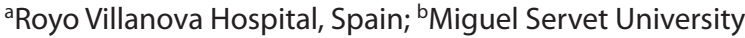
Hospital, Spain; 'Department of Health and Consumer Affairs of Aragón, Spain

Background: While stroke mortality in Spain has decreased significantly in the last decade, trends in stroke hospitalisation and case fatality are less known.

We sought to evaluate trends in stroke hospitalisation and case fatality in a Spanish region (Aragón) over a period of 13 years.
Methods: We identified retrospectively all stroke patients admitted to acute care hospitals in Aragon between January 1, 1998, and December 31, 2010, through the Minimum Basic Data Set - Health Service of Aragon.

The major inclusion criterion was an admission with a principal diagnosis of acute stroke as identified through the ICD-9CM system (430, 431, 433.x1, 434.x1, and 436).

Negative binomial regression models were performed for trends analysis.

Results: We recorded 21.581 (77\%) ischaemic strokes (IS), 5.314 (19\%) intracerebral haemorrhages (IH) and 1.127 (4\%) subarachnoid haemorrhages (SAH).

Age and sex standardized hospitalisation rates for IS showed a significant decrease $(9,7 \%$ in men and 5, $4 \%$ in women). Intracerebral haemorrhage rates were highest in 2001 in men and in 2006 in women, with a subsequent decrease till 2010.

28-day in hospital case fatality showed a significant decrease for IS $(16,3$ to $10,7 \%$; $p<0,001)$ but remained stable for haemorrhagic stroke (IH and SAH).

Conclusion(s): There has been a declining trend in stroke hospitalisation rates in Aragon in the period from 1998 to 2010.

Ischaemic stroke case fatality has shown a declining trend during this period. The role of primary prevention and improvement of acute care for stroke may explain in part this decrease.

\section{2}

\section{Examining the Incidence of Traumatic Brain Injury in} New Zealand, the Bionic Study (2009-2012)

\author{
Barker-Collo S. ${ }^{\mathrm{a}}$, Valery F. ${ }^{\mathrm{b}}$, McPherson K. ${ }^{\mathrm{b}}$, Starkey N. ${ }^{\mathrm{c}}$, \\ Theadom A. ${ }^{\mathrm{b}}$ \\ aUniversity of Auckland, New Zealand; b AUT University, New \\ Zealand; 'The University of Waikato, New Zealand
}

Background: Traumatic brain injury(TBI) causes disability and death in young adults and has a significant impact on the injured person, their family, friends, and society. In NZ, annual estimated direct costs of TBI are over $\$ 100 \mathrm{M}$. However, the exact costs of TBI are unknown as its true incidence has yet to be established. This study aimed to establish the true incidence of TBI in New Zealand.

Methods: A prospective population-based TBI register in the total population of Hamilton and Waikato districts(representative of New Zealand) in 2010-2011(173, 208 residents). All new TBI cases were ascertained over 12-months using prospective and retrospective surveillance. Consenting survivors were followed for 1 year. Complete case ascertainment was assured using multiple overlapping sources of information for all hospitalised/non-hospitalised TBI cases (fatal and non-fatal). This included private and public hospitals, emergency departments (e.g. surgery \& neurosurgery departments), CT/MRI records, hospital discharge register, coroner/autopsy records, death certificates, community health services (GP practices, rehabilitation/outpatient clinics), Accident and Medical Centres in Hamilton and Waikato districts, Accident Compensation funding agency and National Health System databases for all fatal/non-fatal TBIs. 
Results: The findings suggest a significantly higher burden for Maori across the life span, with indcidence in males four times the rate suggested internationally. In addition, while the international literature suggests a peak in incidence from age 15-25, the data here suggest an extended peak until age 35 in all New Zealanders. Incidence for Maori far exceeded that suggested by previously published data on TBI hospitalisations for New Zealand.

Conclusion(s): This study examined incidence of TBI in a large and well-defined population. The study provides accurate and nationally representative estimates of TBI incidence, and identified ethnic inequalities in incidence. Data regarding risk factors for incidence, natural course of recovery, and factors influencing early and late outcomes are currently being collated and will be of use to inform policy, resource allocation, planning of relevant prevention, therapeutic and rehabilitation services.

\section{4}

\section{Clinical and Demographic Profile of Childhood and Adolescence Epilepsy in a Tropical Country}

Dalbem J. ${ }^{a}$, Dalbem Â. ${ }^{\text {a }}$ Siqueira H. ${ }^{\mathrm{b}}$, Papaiz-Alvarenga R.M. ${ }^{c}$

anniversidade Federal de Mato Grosso, Brazil; ' ${ }^{\circ}$ Hospital Geral Universitário, Brazil; ' Unirio, Brazil

Background: Epilepsy affects approximately 50 million individuals around the world, $90 \%$ of whom live in developing countries. It is estimated that 2.4 million new cases occur each year worldwide, with around $50 \%$ of these cases occurring in childhood and adolescence.The objective is to recognize the clinical and demographical profile of children and adolescents.

Methods: Cross-sectional study, door-to-door, in the city of Barra do Bugres, state of Mato Grosso, in children and adolescents aged 0-19 years with epilepsy in the year 2011-2012.

Results: Forty-nine children with diagnosis of epilepsy were evaluated, $61.2 \%$ of whom were male with average age of 8.93 years $(5 \mathrm{~m}-18 \mathrm{y})$. In $61.2 \%$ of the patients, the first epileptic seizure occurred within the first two years of life. Generalized epileptic seizures were the most common type, occurring in $73.4 \%$ of cases. In the prenatal and perinatal periods, $49 \%$ of the children had suffered complications such as hypoxia and epileptic seizures. A family history of epilepsy was found in $73.5 \%$ of cases. In $40.8 \%$ of cases, neurological pathologies associated with epilepsy were found, the most common being cerebral palsy and mental retardation. The family income was up to one minimum wage ( $\mathrm{R} \$ 540$; $€$ 217 ) in $51 \%$ of the cases.

Conclusion(s): Epidemiological studies in epilepsy are important, since these studies are able to provide an estimate of the extent of the problem in the population. This work is still in course in order to establish the prevalence of epilepsy in this region.
115

\section{Epidemiology of Epilepsy in Barra Do Bugres, Brazil}

Siqueira H. ${ }^{\mathrm{a}}$, Dalbem J. ${ }^{\mathrm{b}}$, Papaiz-Alvarenga R.M. ${ }^{c}$, Preux P.M. ${ }^{\mathrm{d}}$

${ }^{a}$ Hospital Geral Universitário, Brazil; ${ }^{b}$ Universidade Federal de Mato Grosso, Brazil; ' UNIRIO, Brazil; dUniversity of Limoges, France

There are few articles discussing population-based studies to analyze the frequency of epilepsy in Brazilian populations. Consequently knowledge about the frequency and clinical characteristics of this population is underestimated.

Objective: The aim of this study is to estimate prevalence of epilepsy and define clinical characteristics about epileptic seizures and epilepsy in a Brazilian countryside population.

Methods: A two-phase model prevalence study was conducted: screening by health agents using Limoges questionnaire and diagnostic confirmation in all suspected cases was performed by a neurologist based on a structured questionnaire during period of January to December of 2011 in Barra do Bugres city, Mato Grosso - Brazil.

Results: Was evaluated 7.689 houses totaling 30.132 subjects interviewed by health agents that detected 264 suspect cases of epileptic seizures: 16 non-epileptic cases was identified, 10 individuals had moved from the city before neurological assessment, 76 was children cases and 162 adults with epileptic seizures. Prevalence of epilepsy in this sample was 7.8/1.000 inhabitants and active epilepsy $6.1 / 1.000$. In our sample $93.8 \%$ of patients with epilepsy used toilets situated inside their house, $59.8 \%$ had completed up to two years of education and $22.2 \%$ have declared himself as illiterate. Family history positive for epilepsy was observed in $47.2 \%$ of patients.

Conclusion: Prevalence data of epilepsy shows less impressive than others Latin America and even some Brazilians' studies. Despite being a Brazilian' rural area, sanitary conditions and socioeconomic characteristics of this population could be a factor responsible for low frequency of epilepsy.

\section{8 \\ Clinical Assessment of Subjects with Idiopathic Normal Pressure Hydrocephalus with MRI Findings in a Community-Dwelling Elderly Population Sample in Japan \\ Nakashita S. ${ }^{a}$, Wada-Isoe K. ${ }^{a}$, Yusuke U. ${ }^{\text {, }}$ Yamawaki M. ${ }^{\text {a }}$, Tanaka K. ${ }^{a}$ Nakashima K. ${ }^{a}$

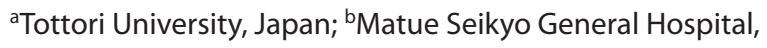 Japan}

Background: It is sometimes difficult to identify idiopathic normal pressure hydrocephalus (iNPH), since the symptoms are often attributed to aging and other neurological disorders. In order to examine the prevalence of possible iNPH on MRI, and clinical backgrounds of subjects having "iNPH on MRI", we con- 
ducted a population-based study in a rural island town in western Japan, Ama-cho.

Methods: Participants included 900 subjects, aged 65 years or older, residing in the town. Six hundreds and ninety-one (76.8\%) of the residents participated in this study. The "iNPH on MRI" was defined as an Evans index of $>0.3$, a narrowing of the subarachnoid space and cortical sulci at the high convexity of the cerebrum. A standardized neurological examination was conducted by board-certified neurologists, including the abbreviated (10-item) version of the motor portion of the Unified Parkinson's Disease Rating Scale (UPDRS). The cognitive impairments were evaluated, using the Mini-Mental State Examination and Clinical Dementia Rating.

Results: Twenty-one subjects (3.0\%) were diagnosed as having "iNPH on MRI". Three subjects $(0.4 \%)$ were diagnosed with possible iNPH under the criteria proposed by the Japanese Society of Normal Pressure Hydrocephalus, five with Alzheimer's diseases, three with mild cognitive impairment, one with Dementia with Lewy bodies, one with vascular dementia, and eight (1.2\%) were asymptomatic of clinical features.

Conclusion(s): A crude prevalence of possible iNPH was $0.4 \%$ in participants over 65 years of age, $1.2 \%$ of participants had no symptoms despite of "iNPH on MRI".

\section{0}

\section{Registry of Bulgarian Patients with Dementia and Mild Cognitive Impairment}

Dimitrov I. ${ }^{\mathrm{a}}$, Milanov I. ${ }^{\mathrm{b}}$, Traykov L. ${ }^{\mathrm{c}}$

aSveta Marina University Hospital, Bulgaria; ${ }^{\text {SSt Naum }}$ University Hospital of Neurology and Psychiatry, Bulgaria;

'University Hospital Alexandrovska, Bulgaria

Background: The design of patient registries in the field of cognitive impairment and dementia should conform to specific professional or social requirements and competences. The necessity to elaborate such registry in Bulgaria has recently become evident, and a protocol was created for the purpose of the first study of dementia and mild cognitive impairment which was conducted in the country.

Methods: A community-based random sample of individuals over the age of 65 years was included in the two-phase, cross-sectional study which was performed in Varna, Bulgaria. The questionnaire included data on demographics, education, professional activities, family status, current health state and medical history, subjective cognitive complaints, motor complaints, dietary habits, alcohol consumption and smoking, use of medicines; a neuropsychological battery, laboratory assessment and imaging were also included. An electronic version of the protocol was created using EpiInfo software, allowing further extension and statistical analysis.

Results: Information according to the study protocol was gathered from 540 subjects who signed the informed consent, out of 605 randomly drawn, and was entered into the electronic database. Thirty-nine patients with different dementia subtypes were initially included in the registry. MCI cases were 36. A total of 465 subjects with normal cognition were also included for future follow-up.

Conclusion(s): No detailed database of patients with dementia and mild cognitive impairment has been created in Bulgaria up to date. The registry elaborated for the purpose of the prevalence study could be used accordingly, being flexible and easy to expand. The collection of supplementary data during patient follow-up could facilitate the assessment of other important epidemiological measures, such as incidence and mortality. It could allow additional analysis to be performed in the future as well.

\section{4}

\section{Are Smoking and Alcohol Use Related to Multiple Sclerosis Severity? A Case-Control Study}

Ivashynka A., Leone M., Lucenti A., Naldi P., Raymkulova O., Ruggerone $S$.

University of Eastern Piedmont, Italy; Maggiore della, Carità Hospital, Italy

Objectives: The influence of smoking on the clinical course of multiple sclerosis (MS) is controversial. We aimed to evaluate the relationship of smoking and alcohol use with the severity of the disease, according to gender.

Methods: We included $236 \mathrm{MS}$ patients with disease duration $>1$ year and age range 20-69 years. Smoking and alcohol histories were ascertained through the European Prospective Investigation into Cancer and Nutrition project questionnaire. Severity of MS was estimated through the Multiple Sclerosis Severity Score (MSSS). Patients were examined at outpatient's department during the follow-up visits.

Results: Women were 154 and men 82. Clinical forms were: 174 relapsing-remitting, 38 secondary progressive, and 24 primary progressive. Drinkers were 185, non-drinkers 51. 138 patients had ever smoked, whereas 98 never smoked. The median MSSS and interquartile range were 2.87 (1.28-5.65), women 3.14 (1.28-5.28), and men 2.34 (1.28-6.24). Median MSSS was lower $(2.82 ; 1.28-5.15)$ for drinkers than for non-drinkers $(3.17 ; 1.77-$ 6.66) Patients that ever smoked had a median MSSS higher (3.17; $1.7-6.0)$ than never smoked $(2.3 ; 1.25-4.96)$. The extreme quartiles of the MSSS score distribution in our patients were $=6$. There were 40 smokers and 43 non-smokers in the lower and 36 and 20 in the highest quartile. Mantel-Haenszel Odds Ratio (ORMH) for smokers after correction for alcohol use and gender was 2.09 (1.03-4.22). There were 69 drinkers and 14 non-drinkers in the lower and 39 and 17 in the highest quartile; the ORMH was 0.42 (0.18-0.99).

Discussion: Smoking is associated with MS severity, whereas alcohol use may be a protective factor. 
131

\section{A Spatial Analysis of Sporadic Amyotrophic Lateral Sclerosis}

Caller T. ${ }^{\mathrm{a}}$, Field $N^{\mathrm{a}}{ }^{\text {, }}$ Shi $X^{\mathrm{b}}{ }^{\mathrm{b}}$, Stommel . $^{\mathrm{a}}$

${ }^{a}$ Dartmouth-Hitchcock Medical Center, USA; b Dartmouth

College, USA

Background: The majority of cases of Amyotrophic Lateral Sclerosis (ALS) are not familial, but sporadic. Detailed epidemiology studies can be used to generate hypotheses for disease etiology and potential contributing environmental factors. In the United States, lack of quality databases has limited detailed epidemiologic investigations. Our objective was to perform a surveillance study of sporadic ALS incidence within Vermont (VT) and New Hampshire $(\mathrm{NH})$, and to determine if there were areas of higher incidence within this region.

Methods: Incident cases of ALS diagnosed 1997-2011 in NH and VT (underlying population 1,942,211 persons) were identified through Dartmouth-Hitchcock Medical Center, Fletcher Allen Health Care, the Muscular Dystrophy Association, and private neurologists. Dwelling addresses at time of diagnosis were geocoded into spatial coordinates using the Google Maps API. Landscan Global 2008 and 2000 US Census data were integrated using ArcGIS $\odot 10$ software and used to create a high resolution background population layer. The expected number of ALS cases was calculated based on expected disease rates and the background population. A Monte Carlo simulation of the distribution of ALS cases was then performed based on the location of actual cases compared to the location of expected cases.

Results: Spatial analysis revealed several regions of statistically significant higher than expected sporadic ALS incidence. Each of these areas contained a minimum of seven cases.

Conclusion(s): Our data suggest that ALS occurs at a higher frequency within certain locations, and environmental exposures could play a role in this geographic variation. Detailed surveillance studies and application of spatial analysis techniques could help further investigation of environmental influences in the development of ALS.

\section{4 \\ Incidence of Guillain-Barré Syndrome (GBS) in Alberta, Canada}

Svenson L. ${ }^{\mathrm{a}}$, Ackah F. ${ }^{\mathrm{b}}$, Sanderson M. ${ }^{\mathrm{b}}$

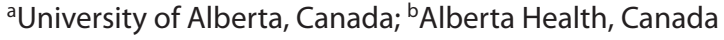

Background: The purpose of the present analysis was to describe the incidence of Guillain-Barré syndrome (GBS).

Methods: The province of Alberta maintains a publicly funded, universally available health care system. Data on all inpatient stays are provided to the Ministry of Health and were used for the present analysis. All in-patient records with an ICD-9 or ICD-10 code for GBS (ICD-9: 357.0; ICD-10: G61.0) were extracted for the period 2000 to 2010. Age-specific and age-stan- dardized rates were computed. The direct method was used to standardize rates to the 1991 Canadian census population.

Results: The province of Alberta maintains a publicly funded, universally available health care system. Data on all inpatient stays are provided to the Ministry of Health and were used for the present analysis. All in-patient records with an ICD-9 or ICD-10 code for GBS (ICD-9: 357.0; ICD-10: G61.0) were extracted for the period 2000 to 2010. Age-specific and age-standardized rates were computed. The direct method was used to standardize rates to the 1991 Canadian census population.

Conclusion(s): The incidence of GBS in Alberta was stable over the time period of study. Incidence increased with increasing age. The incidence in Alberta appears to be among the higher estimates found internationally.

\section{8}

\section{Time of Delay in Stroke Patients of Eastern}

Kadojić D. ${ }^{a}$, Dikanović M. ${ }^{\mathrm{b}}$, Kadojić M. ${ }^{\mathrm{a}}$, Rostohar Bijelić B. ${ }^{\mathrm{a}}$,

${ }^{a}$ Clinical Hospital Center Osijek, Croatia; ${ }^{b}$ General Hospital “Dr. Josip Benčević", Croatia

Background: Thrombolytic therapy improves the outcome after ischemic stroke when applied within 3 hours from symptoms onset. In the past four years we have started preventive campaigns in order to inform the population in Eastern Croatia about the symptoms of stroke and about contemporary therapeutic possibilities for this disease. The aim of this study was to investigate prehospital delay interval after acute stroke and factors that contribute to the late arrival.

Methods: In this prospective one-center study time of delay and main reasons for it were evaluated in 200 patients (96 males and 104 females) admitted to the University Hospital Osijek, Eastern Croatia. Average age of patients was 69.98 years. Time of delay was defined as the time from symptoms onset to hospital arrival. Reasons for delay indicated by patients were divided in three groups: non-recognized symptoms, no knowledge about urgency and late transport.

Results: Mean interval of prehospital delay was 502 minutes, 254 minutes for hemorrhagic stroke, and 542 minutes for ischemic stroke. Time of delay was shortest in patients with subarachnoid hemorrhage (240 minutes) and longest in small vessel stroke (804 minutes). Among investigated patients, $40 \%$ arrived within 3 hours, and another $13 \%$ in the next 3 hours, i.e. 53\% within 6 hours. The most frequent reason for delay was non-recognized symptoms $(52.5 \%)$, followed by late transport $(33.5 \%)$ and no knowledge about urgency (14\%).

Conclusion(s): Our results still show significant prehospital delay for acute stroke patients in Eastern Croatia. This emphasizes the requirement of more effective public health activities and more efficient urgent medicine system, which should reduce time of delay and improve the outcome. 
140

\section{The Survey About the Epidemic of Rampant Cancers in Tehran Province by Geographical Information System}

Ighanian B., Tavakoli A.

GIS activities, Iran

Background: Geographical Information System is a computerized system for managing and analyzing the geographic information. It has the capability to gather, save, analyze and show the geographic information.

Methods: Considering utilizing of zip codes has segregation of information in residential usage, the experts of the ministry of health and medical support this method (utilizing zip codes) as the best way to reach to their aims. Digitizing 18 sheets of the plans of Tehran considering various methods of clarification of existent information including 700 thousand informational records, were analyzed and tested by different softwares like ArcGIS, Arcview, Edvisi, Ilwis and AutoCAD. These methods contain, rechecking the information by operators and clarification of information on the postal and geographical plans by mentioned soft -wares. As the addresses in the files of The Office of Codifying were edited in three parts (sector, penultimate pass way, last pass way). The program firstly verifies all three fields with data bank of cancer to attribute 5 digits codes to patient's address, and then if two fields in one record are verified with data bank, 5 digits of zip code is attributed to the patient's address and finally all records which have received codes are eliminated thus these records are moved out of the comparing circle.

Results: The plans show that the most aggregated zone of cancer epidemy is Bazar and south west is the second most aggregated zone.

Conclusion(s): This method can be use in several cases such as EOC GIS (Emergency Operation Center), Sustainable development, health GIS, Crime GIS, Educational management, Economical cases and any other macro managements system.

141

Sex Differences in Stroke Incidence and 28-Day Case Fatality Rate in a Basic Health Area of Extremadura, Spain, 2000-2009

Ramírez-Moreno J. ${ }^{\text {, }}$ Hernández-Ramos F. , Felix-Redondo F. ${ }^{\mathrm{b}}$, Lozano-Mera L. ', Millan-Nuñez V. ${ }^{\mathrm{d}}$, Fernández-Bergés D. ${ }^{\mathrm{e}}$

${ }^{\text {aH }}$ ospital Universitario Infanta Cristina, Spain; ${ }^{b} \mathrm{C}$. S. Villanueva Norte., Spain; 'Centro de Salud Urbano1, Spain; ${ }^{\mathrm{d}}$ Hospital Universitario Infanta Cristina, Spain; ${ }^{\mathrm{H}} \mathrm{Hospital}$ Don Benito, Hospital Don Benito, Spain

Background: In Spain, stroke is one of the major causes of death and the main cause of severe disability in people over 65 years. Few studies have examined the impact of sex across different age groups and over time on stroke. The objective of this study was to explore the effect of sex across different age groups and over time on stroke incidence and 28-day case fatality in a rural area of Extremadura, Spain.

Methods: Over 2600 first hospitalizations for stroke in this area (from 2000 to 2009) were identified from the Minimum Basic Data Set of the Hospital Register. The 28-day mortality was derived from the linked national death register. Age-specific rate ratios (RRs; men to women) for incidence of first hospitalization and the 28-day case fatality were estimated based on Poisson regression models after adjustment for year of hospitalization.

Results: Over a ten year period, 2287 (82, 8\%) ischemic strokes, $433(15,7 \%)$ cerebral hemorrhages and $41(1,5 \%)$ subarachnoid hemorrhages were registered. Men below the age of 85 years had a higher incidence of first hospitalization that women, and the effect size varied with age [35-44 years: $R R=1,29,95 \%$ confidence interval $(\mathrm{CI})=0,75-2,24 ; 45-54$ years: $2,34,1,51-3$, 62 ; 55-64 years: $2,35,1,82-3,02 ; 65-74$ years: $1,75,1,51-2,03$; $75-84$ years: $1,26,1,12-1,42)]$. Women aged $\geq 85$ years had a significant stroke incidence higher that men $(0,69,95 \% \mathrm{CI}=0$, $55-0,85)$. And the adjusted 30-day mortality was significantly higher than that of men aged $>85$ years $(R R=0,59,95 \% C I=0$, 49-0, 73).

Conclusion(s): Higher rates of incidence were observed in men than women aged 35-84 years in a rural area of Extremadura, Spain; and interestingly short-term mortality is greater in women aged $>85$ years.

\section{6 \\ Obesity and Risk of Parkinson's Disease in a Prospective Study}

Van Den Eeden S., Shan J., Quesenberry C.

Kaiser Permanente, USA

Background: The association between obesity and risk of Parkinson's disease remains unclear. The objective of this study was to examine the risk of Parkinson's disease (PD) associated with obesity in a prospective cohort study.

Methods: We studied a cohort of individuals who were members of Kaiser Permanente Northern California (KPNC) after 1995 and had participated in Multiphasic Health Checkup (MHC) Cohort exam in 1964-1973 ( $\mathrm{n}=66,438)$. Cases were determined by review of KPNC electronic clinical records for neurologist diagnosed PD $(n=1,154)$. Body mass index $(B M I)$ was calculated from the height and weight determined at baseline questionnaire on the entire cohort. Covariate data on age, sex, race/ethnicity, and smoking were also obtained from the MHC. Cox proportional hazard models were used to estimate the hazard ratio (HR) and $95 \%$ confidence interval $(95 \% \mathrm{CI})$ for PD associated with quartile of BMI.

Results: The mean follow-up time for the full cohort was 38.8 years and included over 2,575,000 person-years of follow-up. Cohort members had a mean age at baseline of 36 years, while the cases were approximately 70 years old at diagnosis. Relative to the lowest quartile of BMI, we found no association between quartile 2 or 3 and PD. However, there was a small elevation in risk for quartile 4 (highest) relative to quartile 1 ( $\mathrm{HR}=1.25$, 95\% CI $1.02-$ 1.54). 
Conclusion(s): These prospective data suggest that the highest levels of BMI are associated with an increased risk of Parkinson's disease.

\section{7 \\ Is Dizziness Always Covering a Serious Illness?}

Varga C., Nagy F., Drubics K., Varga-Győrfi K., Papp Z., Lelovics Z., Oláh T.,

"Kaposi Mór"Teaching Hospital, Kaposvár, Hungary

Dizziness is one of the most common symptoms at doctor's office, while setting up the diagnosis is a serious challenge for the medical practitioner. Many factors complicate the correct diagnosis. Patients often use different words to describe the feeling of complex dizziness; in other hand they often use vertigo to describe other changing, subjective, mostly unpleasant experiences or psychical state. Epidemiological characterization of patients with compliant of dizziness at the emergency department. (this section outlinesimportance, objectives/aims or hypothesis of the study).

Retrospective analysis of 471 (29.3\% males) cases of patients with dizziness from the emergency department. $24.0 \%$ of the patients from the emergency department, $76.6 \%$ were ambulatory treated. $46.1 \%$ were transported by the NRS, $53.9 \%$ were outpatients ( $10.6 \%$ alone, $43.3 \%$ with someone else).

Distribution according to GCS: 15 : $98.5 \% ; 14: 1.3 \%$ and 13 : $0.2 \%$. In $43.9 \%$ CT scan were made. The result of the cranial CT scan: $45.9 \%$ negative, $39.6 \%$ old damage, $9.7 \%$ known damage, with the rate of observed changes, 3.9\% new damage and $1.0 \%$ other. Cranial CT scan were made more often in older patients than in younger $(\mathrm{p}=0.016)$ and also in patients who arrived with headache $(\mathrm{p}<0.001)$. Significantly less $(\mathrm{p}=0.021)$ cranial CT was if dizziness was known previously. $56.9 \%$ had nausea besides dizziness, $43.7 \%$ had headache, $31.4 \%$ were vomiting, $18.5 \%$ had arrhythmia and syncope occurred in 21 cases. $81.3 \%$ went home, $7.2 \%$ were taken to the neurology and $5.7 \%$ to the otolaryngology department.

The connection between triage category and making CT scan was significant $(\mathrm{p}=0.016)$, while GCS $(13,14$ and 16$)$ did not affected it $(\mathrm{p}=0.356)$. The nature $(0.081)$ and also the duration (0.350) of dizziness had no significant correlation with the making of CT scan.

\section{0 \\ High Prevalence of Multiple Sclerosis in Shahriar Hospital}

Qorbani M.

Shahriar Hospital, Social Security Org, Iran

Background: Patients with multiple sclerosis (MS) have different prevalence in different regions and jobs.

The objective of our study was to describe the reasons of prevalence of MS is rated so high in Shahriar Hospital, Shahriar, Iran.
Methods: The survey was done among 7828 healthcare workers which were of 23 different facilities in Tehran province. A review of the scientific literature and analysis of information of MS prevalence were used to compare the number of patients in this hospital and other similar health care facilities in Tehran province with comparison to normal population of Shahriar and Tehran region. The patients were evaluated by interview and a questionnaire about the risk factors and the medical history of their disease.

Results: Prevalence of MS was $1.33 \%$ (6 in 450) and all patients were asked to complete questionnaire (response rate: 6 of 6 was 100\% in Shahriar hospital).These items was evaluated between them and normal population and other health facility workers in Tehran province: Age/Month of birth/Gender/family history/ Infections (Epstein-Barr virus). viral exanthemas. Varicella zoster virus. Hepatitis B vaccine/Race and Ethnicity/ Living in temperate climates/other autoimmune diseases/History of severe trauma/History of major surgery/History of pregnancy/ Major anxiety disorder/Major depression/Attendance in war/ smoking/Other neurological disease/First sign and symptom/ Estrogen and Oral Contraceptives/Beginning date /work in shahriar hospital /education/Needle stick injury. There was no meaningful difference between them and others. MS rates were highest among our facility (Shariar hospital) that may be related to water source. The conditions of tests and surveys were similar for all the regions and facilities, but nevertheless this facility (Shariar hospital) had the highest rate among all. The only difference have been noticed between the facilities was water source which at Shahriar hospital has its own private well. Hazardous material in source of water was suspected to be the reason of the problem.

Conclusion(s): It must be studied further more environmental and occupational factors that may be effect on beginning of the disease or accept a hypothesis of accidental gathering of patient in a same place.

\section{5 \\ Challenges Posed to Large Prevalence Studies on Medication Overuse Headache in Scandinavia}

\section{Westergaard M.L.}

Danish Headache Center, Glostrup Hospital, Glostrup, Denmark

Background: Several large studies on medicaton overuse headache $(\mathrm{MOH})$ have been published in Scandinavia in recent years, most notably: a cross sectional study of 44, 300 randomly selected individuals in Sweden interviewed in a national telephone survey (Johnsson, Hedenrud and Linde, 2011), and an 11-year follow up study of 25, 596 persons in Norway (Hagen, Linde, Steiner, Stovner and Zwart, 2012). The objective of this study is to review the methodologies used to estimate the prevalence of $\mathrm{MOH}$ in Scandinavian countries, and to examine potential sources of bias and confounding in the analysis of data available in Denmark.

Methods: This study used data gathered from the Danish 2010 health survey which targeted 129, 150 persons in Region Hovedstaden and Region Sjælland. The comprehensive questionnaire focused on chronic disease, lifestyle factors affecting health, 
and socioeconomic status. Several questions on headache and its treatment with over-the-counter (OTC) analgesics have been included, allowing a presumptive (but not conclusive) diagnosis of $\mathrm{MOH}$. Data on use of prescription medications among those with frequent headache were accessed from the Danish National Prescription Registry.

Results: Analysis of data from Denmark is currently underway, with 68,518 respondents to the survey questionnaire (53\% of targeted). Preliminary results estimate an $\mathrm{MOH}$ prevalence of $2.0 \%$, not unlike the $1.8 \%$ reported in Sweden. Reporting bias and the assumption of causality may lead to over-estimation of prevalence, whereas lower response rates among those with low socioeconomic status may lead to under-estimation. Misclassification of exposure and/or disease are important consideratons.

Conclusion(s): Attempts to estimate the prevalence of $\mathrm{MOH}$ must acknowledge the challenge of establishing a case definition that is useful for population-based studies. There are biases in the different strategies employed to gather data, leading to both overand under-estimation of prevalence. Preliminary data from Denmark show a prevalence of $\mathrm{MOH}$ that is similar to the $1-2 \%$ prevalence reported in global studies. There may be limitations in the generalisability of results despite the large numbers of participants in the Scandinavian studies due to the relatively ethnically and culturally homogenous western European study populations. Data from health registries and prescription drug databases in Denmark (as well as Sweden and Norway) can be optimally used for $\mathrm{MOH}$ research.

\section{6 \\ Environmental Risk Factors and Rural Living in Parkinson's Disease: A Patient's Perception}

Daunt L., Sharma J., Aw D.

United Lincolnshire Hospitals, United Kingdom

Background: Environmental factors have been found to be associated with developing Parkinson's Disease. In particular exposure to pesticides, chemicals, farming and rural living. We wanted to investigate the environmental exposures of patients attending a Parkinson's Disease clinic at a district general hospital in a rural setting.

Methods: Retrospective, anonymous purpose designed questionnaire was distributed in clinic to patients with diagnosed Parkinson's Disease.

Results: Fifty patients were surveyed, with a mean age of 70 years, $60 \%$ were male. Two thirds of patients considered themselves to be living in a rural location. One quarter felt they had previously been exposed to pesticides and another quarter felt they had been exposed to industrial chemicals, including detergents, solvents and paints. All of these chemical exposures were related to occupation, although $75 \%$ of patients were now retired. Over $90 \%$ of patients felt exposure to pesticides was related to living in a rural setting. $39 \%$ of patients surveyed had worked with farm animals as a result of rural living, despite only one patient being a farmer by occupation. Overall, half of patients felt that past exposures were in some part to blame for their condition.

Conclusions: In this small survey, our findings have demonstrated that rural living is associated with increased exposure to pesticides and farm animals, which may predispose to developing Parkinson's Disease. Patients perceive that past exposures have a part to play in disease causality. In addition, some occupations are associated with chemical exposure and measures should be taken to reduce the risk of harm.

\section{8 \\ OFSEP: Observatoire Français de la Sclérose en Plaques}

\author{
Vukusic S. ${ }^{a}$, Clanet M. ${ }^{\mathrm{b}}$, Confavreux C. ${ }^{\mathrm{a}}$
}

aHôpital Neurologique et Fondation EDMUS pour la Sclérose en Plaques, Toulouse, France; b Hôpital Purpan, Toulouse, France

In January 2011, the "Observatoire Français de la Sclérose en Plaques - OFSEP" project was selected by the French Ministry of Higher Education and Research after the call for proposals "Cohortes 2010" issued by the Agence Nationale de la Recherche (ANR), providing a continuous grant for ten years.

The care of MS by neurologists in France has several unique strengths:

(1) a structuration based on two complementary and closely interlinked networks: 28 referral MS centres in university hospitals and 18 regional "city-hospital health networks";

(2) the availability of a single databasing system, EDMUS, fully integrated in daily practice, allowing a comprehensive description of all aspects of MS;

(3) the availability of a nationwide cohort of more than 35.000 patients with MS (PwMS) registered at the EDMUS format;

(4) the breakdown, within this large 'mother' cohort, of nested cohorts: natural history, childhood onset MS, neuromyelitis optica, patients treated with natalizumab;

(5) the availability of two Biological Resource Centres (BRCs): REFGENSEP, entirely dedicated to the collection of genetic material from PwMS, and NEUROBIOTEC.

With this funding, the OFSEP will be able to increase the size of the national cohort and will allow extending its scope of investigations, combining clinical data with biological samples and imaging data. This selection should also enable the project to implement pharmaco-epidemiological studies in collaboration with regulatory authorities and the industry.

By supporting OFSEP, the Ministry provides the country with a powerful epidemiological tool and an "MS plan" which are unique in the world. 


\section{1}

\section{Severe Head Injury and Amyotrophic Lateral Sclerosis}

Peters T. ${ }^{\mathrm{a}}$, Fang F. ${ }^{\mathrm{a}}$, Weibull C. ${ }^{\mathrm{a}}$, Sandler ${ }^{\mathrm{b}}{ }^{\mathrm{b}}$, Kamel F. ${ }^{\mathrm{b}}$, Ye W. ${ }^{\mathrm{a}}$

${ }^{a}$ Karolinska Institutet, Sweden; ${ }^{b}$ National Institutes of Health, USA

Background: Previous studies on the association between head injury and risk of amyotrophic lateral sclerosis (ALS) have provided inconsistent results. The objective of this study was to examine whether severe head injury, subtypes of head injury, or repeated head injuries are associated with ALS risk based on the Swedish population and health registers.

Methods: We conducted a case-control study, nested within a cohort of 5, 764, 522 individuals who were born in Sweden during 1901-1970 and followed up between 1991 and 2007, that included 4, 004 ALS patients identified from the Swedish Patient Register during follow-up and 20,020 randomly selected controls matched by sex and birth year (1:5 matching). We evaluated hospitalization for severe head injury before ALS diagnosis. Conditional logistic regression was used to estimate odds ratios (ORs) and 95\% confidence intervals (CIs).

Results: There was an association of ALS risk with severe head injury $\leq 1$ year before diagnosis (OR: 3.9, 95\% CI: 2.6-6.1). No association was observed for severe head injury $>1$ year before ALS diagnosis. Considering subtypes of head injury and repeated injuries occurring $>3$ years before diagnosis did not further modify the association.

Conclusion(s): Our findings from the Swedish registers provide little support for an etiological relationship between severe head injury in adulthood and ALS risk.

\section{1 \\ Nationwide Epidemiological Study of Pediatric Neurotransmitter Disease in Japan}

Shintaku $H$.

Osaka City University Graduate School of Medicine, Japan

Backgroud: Pediatric neurotransmitter disease (PND) is a relatively new concept in medical science. PNDs, which are induced by genetic disorders that affect the regulation of neurotransmitters in children, include dopamine-/serotonin-related diseases and GABA-related diseases. Left untreated, PNDs can lead to severely compromised neurological function in patients. However, diagnosing these diseases has been difficult. Thus, it is assumed that many of those patients were not diagnosed accurately and they did not receive appropriate treatments. We investigated the numbers and the distributions of patients with these diseases in Japan.

Methods: We sent a questionnaire to pediatricians or neurologists of 1622 Japanese hospitals in 2009. Between 2010 and 2011 we measured metabolites of neurotransmitters and pteridines in cerebrospinal fluid and confirmed the diagnosis by DNA analysis of these PNDs.

Results: We received replies from $60.3 \%$ of those hospitals. In dopamine-/serotonin- related diseases, 116 patients of Segawa disease (autosomal dominant guanosine triphosphate cyclohydrolase I (GTPCH) deficiency) were reported from 44 hospitals. Those patients were in every 10 region of Japan. The prevalence rate of Segawa disease was calculated as 0.96 patients/ million. Three patients of aromatic-L-amino acid decarboxylase (AADC) deficiency, another dopamine- /serotonin- related PND, were also reported from 2 hospitals. No patient with tyrosine hydroxylase (TH) deficiency or sepiapterin reductase (SR) deficiency was reported in this study. In GABA-related disease, 3 patients of succinic semialdehyde dehydrogenase (SSADH) deficiency were reported from 3 hospitals. In 2011 we found total 160 patients with Segawa disease, 3 patients with AADC deficiency, and 4 patients with SSADH deficiency.

Conclusion: The prevalence rate of Segawa disease in this study was similar to that of a previous report by Nyggard et al. in 1993 (0.5-1.0 patients/ million). However many patients with Segawa disease were newly discovered by creating diagnostic criteria and advancing diagnosis positively based on it.

\section{5 \\ Does Hypercholesterolemia Relate to Mortality? Results from the NEDICES Cohort}

Sierra-Hidalgo F., Trincado R., Sánchez-Ferro Á., Díaz-Guzmán J., Martínez-Salio A., Hernández-Gallego J., Bermejo-Pareja F.

H. U. 12 de Octubre, Spain

Background: Cholesterol is a well-documented risk factor for cardiovascular mortality, but data on hypercholesterolemiarelated mortality among the elderly are limited. The aim of the present study was to investigate the relationship between high serum cholesterol levels and mortality among a population of older adults.

Methods: The Neurological Disorders in Central Spain (NEDICES) study was a prospective population-based cohort study with a follow-up of 13 years. Elderly subjects ( $>$ / = 65 years old) were assessed for self-reported hypercholesterolemia and lipid-lowering therapy at baseline. Mortality and its causes were assessed over 13 years of follow-up using data from the Spanish Mortality Register. Cox proportional hazard models and logistic regression models were conducted in the total sample and within age groups.

Results: The final sample included 4633 subjects with $29.1 \%$ prevalence for hypercholesterolemia at baseline. Mortality rate was higher among subjects without hypercholesterolemia than among those with hypercholesterolemia (73.4 per 1, 000 personyears vs 30.1 per 1, 000 person-years). In Cox-proportional hazard models adjusted for sex, age and other potential confounders, an increased mortality risk was found to be related to male gender and older age. Mortality risk was lower among subjects with known hypercholesterolemia at baseline independent from treatment status (HR 0.87, 0.78-0.97 95\% CI). An increased risk of 
mortality caused by ischemic heart disease was found in hypercholesterolemia (OR 1.87, 1.31-2.67 95\% CI).

Conclusion(s): Long-term mortality rate is lower among subjects with hypercholesterolemia when compared to non-hypercholesterolemic subjects, although ischemic heart disease-related mortality was higher among the former.

\section{8}

\section{Is There an Association Between Varicella Immunization and Stroke?}

\author{
Svenson L. ${ }^{\mathrm{a}}$, Dover D. ${ }^{\mathrm{b}}$, Hill M. ${ }^{\mathrm{c}}$ \\ aniversity of Alberta, Alberta, Canada; ${ }^{\text {b} A l b e r t a ~ H e a l t h, ~}$ \\ Canada; 'University of Calgary, Canada
}

Background: Stroke is known to be associated with varicella disease. The purpose of our study was to assess the risk of stroke following receipt of varicella vaccine.

Methods: The province of Alberta maintains a publicly funded, universally available health care system. As part of managing the system, all residents of the province are assigned a personal health number (PHN), which is a unique lifetime identifier, that is used to manage health services. A population-based registry contains demographic details on the population and was used to create two cohorts. The first was all individuals with birth dates between 1996 and $1999(\mathrm{n}=138,115)$ or between 2006 and 2009 $(\mathrm{n}=179,126)$. The follow-up periods were to March 31, 2001 and March 31, 2011 for cohort one and cohort two, respectively. Using the PHN both groups were linked to population level immunization event data for varicella, hospital inpatient data, and physician fee-for-service data. The date of immunization was captured as well as the date of any chickenpox diagnosis. Inpatient data were used to determine if the child had had a cerebrovascular incident using ICD-9 (362.3, 433.x1, 434.x1, 436, 431.x, 430.x, 435.x) or ICD-10 codes (H34.1, H34.2, I63.x, I64.x, I61.x, I60.x, G45.x, H34.0).

Results: The number of children receiving varicella vaccine was higher among those in the second cohort (cohort 1: $0.4 \%$; cohort 2: $83.7 \%)$. A history of having had chickenpox was higher for cohort $1(6.4 \%)$ than cohort $2(0.7 \%)$. In cohort $1,0.04 \%$ ( $=$ 52) were diagnosed with a stroke compared to $0.06 \%$ for cohort 2 $(\mathrm{n}=105)$. Age was a significant predictor of the occurence of stroke. Using those aged 6-17 months as the reference, children aged 1 day to 6 months were significantly more likely to have had a stroke $(R R=15.68, p<.05)$. There was no statisically significant increased risk of having a stroke up to two years post immunization.

Conclusion(s): Receiving varicella vaccine does not appear to be associated with an increased risk of cerebrovascular disease.
189

Incidence of Primary Brain Tumours Among the Adult Population of Emilia Romagna Region, Italy

D'Alessandro R. ${ }^{a}$, Albani F. ${ }^{a}$, Ferro S. ${ }^{a}$, Baldin E. ${ }^{a}$, Testoni S. ${ }^{\text {, }}$ de Pasqua S. ${ }^{a}$, Brandes A. ${ }^{\text {b }}$, Baruzzi A. ${ }^{\text {a }}$

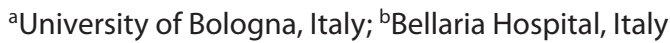

Background: There are no prospective studies on the incidence of PBT among large populations in Italy.

Methods: Specialists in neurology, neuroradiology, neurosurgery, oncology, radiotherapy, biomolecular and anatomical pathology, and genetics throughout the Region were invited use the project website (www.perno.it) to notify all new adult cases of suspect PBT observed from 1st January 2009 and living in the Emilia Romagna region. Periodic reminder were sent to all participants. Clinical findings allowing the final diagnosis were then collected from original clinical records. ICD discharge codes from hospitals of Region and neighbouring areas from 1st January 2009 to 31st March 2010 were also reviewed to retrieve cases previously non notified. PBT diagnosis was based on histological confirmation or typical radiological findings with no evidence of cancer elsewhere.

Results: Four hundred and sixteen cases (239 men, 177 women) of PBT were recorded from 1st January to 31st December 2009. Of these 109 were identified from ICD discharge codes. Histological confirmation was obtained in 236 cases (56\%). Of these, 151 (63\%) were diagnosed as glioblastoma (GBM) and 85 as other neuroepithelial tumours (37\%). One hundred and eighty patients had a clinical and radiological diagnosis only. Of these, $124(66 \%)$ showed features of high grade gliomas. Patients identified from ICD codes were older ( 73 vs 63 years) and more often women (50\% vs 40\%). The PBT 2009 incidence rate/100, 000 persons/year was 10.4 (95\% CI: 9.4-11.5), higher in men (12.4 95\%CI: 10.9-14.1) than in women (8.6 95\%CI: 7.4-10).

Conclusion(s): Our PBT incidence rates are among the highest reported. GBM is the most common tumour type. Elderly patients and women may be underreported in prospective studies. 
192

\section{Feasibility of Population Based Stroke Registry in Ludhiana City, India}

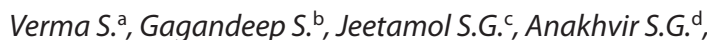
Sobti M.K. ${ }^{\mathrm{e}}$, Arora O.P. , Sehgal H. ${ }^{\mathrm{g}}$, Dhanuka A.K. ${ }^{\mathrm{h}}$, Pawar S.S.', Saxena A. ', Kaur M. ${ }^{\prime}$, Bakshi S.S.', Paramdeep K. ${ }^{a}$, Gagandeep M. ${ }^{\text {, }}$, Amber S. ${ }^{a}$, Singh Y. ${ }^{a}$, Meenakshi S. ${ }^{m}$, Grover A. ${ }^{m}$, Shah $B .{ }^{m}$, Pandian J.D. ${ }^{\mathrm{a}}$

${ }^{a}$ Christian Medical College and Hospital, India; ${ }^{b}$ Dayanand Medical College and Hospital, India; 'Delta Heart Centre, India; ${ }^{\mathrm{d} H e a r t}$ Beat Diagnostics Centre, India; ' Sobti Neuro and Super Speciality Hospital, India; ${ }^{\text {A Arora Neuro Centre, India; }{ }^{9} \text { Guru Teg }}$

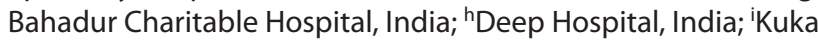
Diagnostic Centre, India; 'Mediways Hospital, India; ${ }^{\text {KShree }}$ Raghunath Hospital, India; 'Ace Health Ways, India; ${ }^{\text {mNon }}$ Communicable Diseases Division Indian Council of Medical Research, India

Background and Aims: Population based incidence studies on stroke from developing countries are scarce. Hence we carried out this study to evaluate the feasibility of establishing a population based stroke registry and to collect the methodology for data collection in Ludhiana city.

Methods: The population of Ludhiana city is 1, 398, 467 and people over 18 years as per 2007 data is 935,925 . The data collection was done from August 2010 till August 2011. The WHO STEPS methodology was used with modifications. The trained research staff visited the centres and collected stroke information.

Separate questionnaires were used for hospital, scan centres and to collect stroke deaths from Municipal Corporation Office. Verbal autopsy was done by door-to-door survey on $20 \%$ of non stroke deaths. Outcome was assessed using modified Rankin scale (mRs) by telephonic interview at 1 month.

Results: 964 patients were identified from Ludhiana city. The problems encountered were as follows: Reluctance to share data due to privacy issues; difficulty in capturing minor strokes from the general practitioners; difficulty in identifying key person from each centre; loss of medical records files from public hospitals; frequent change of mobile numbers by subjects; difficulty in conducting verbal autopsy due to incorrect addresses; poor documentation of deaths in hospitals.

Conclusion: Population based stroke registry is feasible in an urban population with the above methodology. The problems were identified and corrective measures were implemented accordingly in each centre.

Acknowledgements: Indian Council of Medical Research Task Force Project or future research is briefly described).

\section{3 \\ Physical Activity Is a Protective Factor for Dementia. Data from NEDICES Cohort}

Bermejo-Pareja F.a , Medel A. benito-León J. ' , Siebel P. , , HerreroSan Martín A. ${ }^{\mathrm{C}}$

${ }^{\mathrm{a} H}$. U. 12 de Octubre and CIBERNED, Spain; ${ }^{\mathrm{b} C i b e r n e d, ~ S p a i n ; ~}$ 'H. U. 12 de Octubre, Spain

Background: To analyse if physical activity (PA) could be a protective factor for dementia incidence in the NEDICES (Neurologic Disorders in Central Spain) elderly cohort.

Methods: The baseline survey (1994-5) in NEDICES cohort evaluated 5, 278 census-based participants. General health and life style were assessed in the basal survey, included PA with a modified Breslau-Rosow questionnaire and its scores permitted to graduate the PA in three groups (sedentary, and moderate and high PA). After a mean follow-up of 3.2 years we estimated the dementia incidence (1997-8). Several multivariate statistical analyses were performed to evaluate if PA is a protective factor for dementia incident cases, adjusted by age, sex, education and other dementia RF in this cohort (previous cognitive performance and previous stroke).

Results: 161 incident dementia cases were identified among 3, 891 individuals assessed at follow-up. The large majority had AD: 115 (71.4\%) (J Neurol Sci, 2008, 264:63-72). We were able to evaluate PA in 3, 047 participants and 129 incident dementia cases. In Cox regression, the HR of moderate PA group versus the sedentary group was $0.62[(95 \% \mathrm{CI})=0.40-0.96] ; \mathrm{p}<.03$. There was no clear PA dose effect between the moderate and high PA group.

Conclusion(s): In te NEDICES cohort, sedentary physical activity is a risk factor of incident dementia. This result indicates the need of more investigations in this subject.

\section{0}

\section{The Association Between Depression and General Hospital Outcomes: A Community-Based Data Linkage Study of Older Men}

Prina M. ${ }^{a}$, Huisman M. ${ }^{b}$, Carol B. ${ }^{a}$, Almeida O. ${ }^{c}$

a University of Cambridge, United Kingdom; 'bU University, The Netherlands; ' ${ }^{C}$ nniversity of Western Australia, Australia

Background: Studies that have investigated the relationship between depression and the type, nature, extent, cost and outcome of general hospital admission are limited by their retrospective designs and focus on specific clinical populations. We aimed to explore this relationship prospectively in a large, communitybased sample of older men.

Methods: A cohort of 5411 older men enrolled in the Health in Men Study(HIMS) was assessed at baseline for depressive symptoms, as defined by a score of 7 or higher on the Geriatric Depression Scale (GDS), and followed-up for two years for the occurrence and number of hospitalisations, types of hospital admission, length of hospital stay, in-patient deaths, and associated costs as recorded by the Western Australian Data Linkage System. 
Results: $75 \%$ of 339 men with depressive symptoms had at least one hospital admission compared to $61 \%$ of 5072 nondepressed men $(\mathrm{p}<0.001)$. In multivariate analyses, the presence of depressive symptoms was a significant, independent predictor of hospitalisation (hazard ratio [HR]: $1.31,95 \%$ confidence interval [CI]: 1.14-1.51), number of hospitalisations (rate ratio [RR]: 1.48, 95\% CI: 1.30-1.69), total length of hospital stay (RR: 1.88, 1.60-2.22), hospital in-patient mortality (HR 2.30, 1.48-3.56) and higher costs (RR 1.44, 1.23-1.68).

Conclusion(s): Depressed older men were at higher risk of hospitalisation for non-psychiatric conditions, and were more likely to have more prolonged and costly hospitalisation and worse hospital outcomes, compared to non-depressed adults. These results highlight the potential to target this high-risk group of individuals to reduce the burden of health-care costs in an ageing population.

\section{8}

\section{Epidemiology of Epilepsy in Egypt}

Farghaly W. ${ }^{a}$, Nagiub H. ${ }^{a}$, Rageh $T^{a}$, Shehata G. ${ }^{a}$, Badry R. ${ }^{a}$, Badry N. ${ }^{b}$, Abdelmonem M. ${ }^{c}$, Abd Al Wareth A. ${ }^{a}$, Kandil M. ${ }^{\text {a }}$

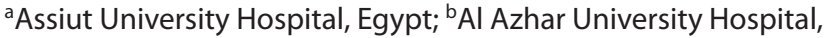
Egypt; 'Sohag University Hospital, Egypt

Background: Epilepsy is a common childhood neurological disorder throughout the world. Types of epilepsy among children, causes and prognosis, differ widely from epilepsy in adulthood and elderly. Epilepsy has serious impact on cognitive development and social activities, and might adversely affect quality of life not only of the child with epilepsy, but also of the whole family members. The objective of this study is to illustrate epidemiology of epilepsy among children in AlQuseir city- Red Sea Governorate and to estimate the magnitude of its treatment gap.

Methods: The study was a part of a door to door study, including every door to screen all inhabitants in Al-Quseir city $(33,285$ inhabitants; out of whom 12, 093 were children below 18 years old); by 3 specialists of neurology and 15 females social workers (for demographic data collection) using standardized screening Quesnnaire (Eltallawy et al, 2010). All suspected cases were subjected to detailed history, clinical examination, EEG, Neuroimaging studies, and estimation of serum drug level if needed.

Results: The study revealed that life time prevalence of epilepsy among children in AlQuseir city is 9/1000 children, with highest peak among neonates and during early infancy (0-2years). The annual incidence rate is $82.7 / 100,000$ children, again with highest incidence rate during the first two years of life and the least during late childhood (12- < 18 years). Prevalence of epilepsy was higher among males (10.5/1000) than female children(7.4/1000). Focal epilepsy is the most frequently encountered type $(58.7 \%)$. Peri-natal complications $(15.6 \%)$ is the most frequently encountered cause of structural/metabolic epilepsy in children. Treatment gap is $84.5 \%$ among Egyptian children with epilepsy in Al Quseir city.

Conclusion: Epilepsy in Egyptian children is underestimated and undertreated.
239

\section{Pattern of Major Neurological Disorders}

Nagiub H. ${ }^{a}$, Farghaly W. ${ }^{\text {, }}$ Rageh T. ${ }^{\text {, }}$ Shehata G. ${ }^{a}$, Badry R. , Badry N. ${ }^{\mathrm{b}}$, El Moselhy E. ${ }^{\mathrm{b}}$, Hassan M. ${ }^{\mathrm{b}}$, Abdelmonem M. ${ }^{\mathrm{c}}$, Hamed Y. ${ }^{\mathrm{b}}$, Abd Al Wareth A. ${ }^{\mathrm{a}}$, Shaaban I. ${ }^{\mathrm{b}}$, Kandil M. ${ }^{\mathrm{a}}$

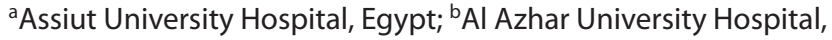
Egypt; 'Sohag University Hospital, Egypt

Background: Epidemiological studies in our country are deficient. In the absence of appropriate systems for medical registry door to door survey stands to be the most suitable method for epidemiologic studies.

Methods: A door- to - door study (including every door) was conducted for all inhabitants of Al Quseir city $(33,283)$, Red Sea Governorate by 3 specialists of neurology as well as 9 senior staff members of neurology and 15 female social workers to assess the epidemiology of major neurological disorders. Through eight phases from July 1st 2009 to Jan 31st 2012, all major neurological disorders included in the study were screened and diagnosed according to the accepted criteria by WHO.

Results: The order of frequency of prevalence of the studied neurological disorders were Dementia $(3.83 \%$ for those aged $>60$ years), Migraine (2.8\% for those aged $>8$ year), Stroke (6.2/1000, for those aged $>20$ years), Epilepsy (5.5/1000), Parkinsonism (452.1/100, 000, > 40 years), Cerebral Palsy (3.6/1000 among children $<18$ years), spinal cord disorders $(63 / 100,000)$ Dystonia (39.11/100, 000), Cerebellar Ataxia (30.01/100, 000), Trigeminal Neuralgia $(28 / 100,000>37$ years), Chorea $(21.03 / 100,000)$, Athetosis $(15 / 100,000)$, and lastly multiple Sclerosis $(13.74 / 100$, 000). The incidence rate of stroke, epilepsy, and Bell's palsy was 98.9/100, 1000 year respectively.

Conclusion: This study has the priority to evaluate the prevalence of Trigeminal neuralgia, Multiple Sclerosis, and spinal cord disorders for the first time in Egypt. There is high prevalence of Parkinson's disease, Cerebral Palsy and Stroke in Al Quseir compared with worldwide studies.There is marked decline in the Prevalence of Rheumatic Chorea in Egypt. 


\section{3}

\section{Short-Term Outcome of Stroke: Data from Ludhiana Population Based Stroke Registry}

Kaur P. ${ }^{\text {a }}$ Shavinder S. ${ }^{\text {b }}$, Shweta J.V. ${ }^{a}$, Gagandeep M. ${ }^{a}$, Amber S. ${ }^{a}$, Yashpal S. ${ }^{a}$, Meenakshi S. ${ }^{\text {, }}$ Ashoo G. ', Bela S. ${ }^{c}$, Mandaville G.D. ${ }^{c}$, Jeyaraj D.P. ${ }^{d}$

aDepartment of Social and Preventive Medicine, Christian Medical College \& Hospital, India; ${ }^{b}$ Division of Non Communicable Diseases, Indian Council of Medical Research, New Delhi, India; 'Sir Ganga Ram Hospital, New Delhi, India; dStroke Unit, Department of Neurology, Christian Medical College and Hospital, Ludhiana, India

Background: Short-term stroke outcome has not been well studied at the population level in developing countries. We carried out this research to find out the short-term outcome at 6 months from the Ludhiana population based stroke registry.

Methods: Population of Ludhiana city is 1, 398, 467 and 935, 925 were over 18 years. All first ever stroke patients ( $\geq 18$ years) between August 2010 to August 2011 were included. Stroke patients data was collected from major hospitals, scan centres and general practitioners, using separate questionnaires. We also collected information regarding recurrence of stroke, details of medications and use of complementary and alternative medicine (CAM). Details of deaths were collected from Municipal Corporation. Verbal autopsy was done in $20 \%$ of non-stroke deaths. The outcome was assessed using modified Rankin scale ( $\mathrm{mRs} \leq 2$ : good outcome) at 6 months by telephonic interview.

Results: 945 stroke patients were enrolled, out of this in 717 (75.8\%) patients' telephone numbers were available. A total of 592 patients were called and $376(63.5 \%)$ responded. The mean age was $58.3 \pm 15.1$ years and $582(61.5 \%)$ were men. The case fatality rate at 6 months was $152(30 \%) .244(64.6 \%)$ patients had good outcome and $27(7.7 \%)$ patients had recurrence of stroke. Only $229(65.6 \%)$ patients remained on secondary prevention drugs. About 62 (17.8\%) patients used CAM.

Conclusion(s): The case fatality rate was $30 \%$. Two-thirds of our patients had good outcome and remained on secondary prevention drugs. CAM was used by $17.8 \%$ of our cohort.

Acknowledgement: This project is supported by the Indian Council of Medical Research (Task Force Project).

\section{6}

\section{Mortality of Subjects with Alcohol-Related Seizures Increased After Alcohol Price Reduction}

Vaaramo K. ${ }^{\text {, }}$ Puljula J. ${ }^{a}$, Tetri S. ${ }^{a}$, Juvela S. ${ }^{b}$, Hillbom M. ${ }^{a}$

aOulu University Hospital, Finland; 'bniversity of Helsinki, Finland

Background: Alcohol-related seizures account for a large part of acute symptomatic seizures and subjects with alcohol-related seizures have an increased risk of death. We investigated whether the reduction of alcohol prices in Finland (March 1st, 2004) asso- ciated with an increase in mortality of subjects with alcoholrelated seizures.

Methods: All subjects with head trauma $(\mathrm{n}=827)$ were identified in Oulu University Hospital during 1999 and thereafter followed until the end of 2009. We used National Hospital Discharge Register, hospital charts and death records from Official Cause-ofDeath Statistics to identify seizure visits, visits due to harmful drinking and alcohol-related deaths during follow-up. KaplanMeier survival curves were used to characterize the effect of alcohol price reduction on risk of alcohol-related seizures and death. Cox proportional hazards model was used to identify independent predictors for new-onset seizures and death.

Results: Mortality of subjects with alcohol-related seizures steeply increased after the price reduction. The proportion of subjects with new-onset seizures was significantly higher $(\mathrm{p}<0.001)$ among harmful drinkers $(21.5 \%)$ than among others (3.6\%) during the follow-up. Harmful drinking (HR 24.74, 95\% CI 10.29$59.46, \mathrm{p}<0.001)$ was the most powerful predictor of new-onset seizures. Age (1.06 per year, 1.05-1.07, $\mathrm{p}<0.001$ ), harmful drinking $(2.47,1.58-3.86, \mathrm{p}<0.001)$, moderate-to-severe index traumatic brain injury $(2.31,1.58-3.38, \mathrm{p}<0.001)$ and seizures (either preceding or after the index trauma) $(1.64,1.07-2.51, \mathrm{p}=0.022)$ were independent predictors of death.

Conclusion: We conclude that the political decision to lower alcohol price associated with a significantly increased mortality of subjects with alcohol-related seizures.

\section{2 \\ Incidence of Transient Ischemic Attack from Ludhiana Population Based Stroke Registry}

Sharma A.

Christian Medical College and Hospital, India

Background and aims: The incidence of Transient Ischemic Attack (TIA) is unknown from developing countries. Hence we carried out this study to find out the incidence of TIA from the Ludhiana population based stroke registry.

Methods: The population of Ludhiana city is 1, 398, 467 and 935, 925 were over 18 years. The study period was from January 2011 to December 2011. All first ever TIA patients ( $\geq 18$ years) were included. TIA was defined as a focal (or at times global) neurological impairment of sudden onset, and lasting $<24$ hours. Patients data were obtained from major hospitals, scan centres and general practitioners using separate questionnaires.

Results: 1279 stroke patients were seen, and out of this 60 (4.7\%) had TIA. The mean age of TIA patients was $58.4 \pm 14.1$ years and $37(61.7 \%)$ were men. The annual incidence rate of TIA was 6.5/100, 000 (95\% confidence interval (CI): 5.3-8.4). The age adjusted incidence rate per 100, 000 was 9.3 (95\% CI: 6.9-11.6); men 10.1 (95\% CI: 6.9-13.4); women 6.9 (95\% CI: 4.1-9.8). Out of 60 patients, $24(75 \%)$ persons had TIA in carotid circulation and 8 $(25 \%)$ patients in vertebrobasilar territory. The common risk factors were: hypertension 25 (80.6\%), diabetes 15 (46.9\%), alcohol 4 (12.5\%) and desi ghee (clarified butter) intake 21 (65.6\%). 
Conclusions: The incidence of TIA was $6.5 / 100,000$ in this study. The average age of our TIA patients was lower than the reports from developed countries. Hypertension was the common risk factor.

Acknowledgement: Indian Council of Medical Research Task Force Project.

\section{4}

Clinical and Polysomnographic Characteristics of 89 Chinese Patients with Idiopathic RBD

Yu H. ${ }^{\mathrm{a}}$, Chen H. ${ }^{\mathrm{b}}$, Yuan L. ${ }^{\mathrm{a}}$, Zhao J. ${ }^{\mathrm{a}}$, Yang G. ${ }^{\mathrm{c}}$, Wang J. ${ }^{\mathrm{c}}$, Hong Z. ${ }^{\mathrm{a}}$

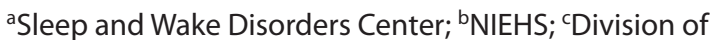
Epidemiology, China

Background: Rapid eye movement sleep behavior disorder (RBD) is a rare parasomnia that may precede the onset of several neurodegenerative diseases. However, little is known about RBD in China.

Methods: We analyzed medical records from 89 consecutive patients with idiopathic RBD diagnosed at the Sleep and Wake Disorders Center of Huashan hospital between May 2010 and July 2012.

Results: Patients were mostly male (85.4\%), and the average age at onset was $60.8 \pm 6.6$ years (duration: $4.6 \pm 3.9$ years). The average total sleep time was $309.6 \pm 56.6$ minutes with a mean sleep efficiency of $72.2 \pm 11.9 \%$, a sleep latency of $20.1 \pm 20.3$ minutes, and a REM sleep latency of $97.3 \pm 54.9$ minutes. The proportion of each sleep stage was $16.4 \pm 7.5 \%$ for $\mathrm{N} 1,47.4 \pm 9.0 \%$ for $\mathrm{N} 2$, $19.2 \pm 8.5 \%$ for slow wave sleep, and $18.1 \pm 11.2 \%$ for REM sleep. Thirty six (40.5\%) patients had periodic leg movements during sleep (PLMS $\geq 15 / \mathrm{h}$ ). The sleep apnea hypopnea syndrome was relatively rare, $5.6 \%$ had an apnea-hypopnea index of $\geq 30$ /hour ( $16.9 \% \geq 15 /$ hour). The multiple sleep latency tests showed $15.4 \%$ $(12 / 78)$ had a short sleep latency ( $\leq 10$ minutes). Six patients $(6 / 67$, $9 \%)$ reported a family history of probable RBD. Several key nonmotor symptoms were common among these iRBD patients: $63.1 \%(41 / 65)$ reported constipation, followed by hyposmia (Sniffing-Stick score $\leq 5$ : 38/68, 55.9\%), daytime sleepiness (Epworth daytime sleepiness score $\geq 10: 17 / 89,19.1 \%$ ) and depression (Beck depression inventory score $\geq 17,13 / 80,16.3 \%$ ). Approximately $37 \%$ of the patients had two or more nonmotor symptoms, and $12.4 \%$ had three or more.

Conclusion(s): This is the first report on clinical characteristics of a large number of iRBD patients in China, and nonmotor symptoms are commonly found.

\section{3 \\ Cerebrovascular Disorders in Myeloproliferative Neoplasms with the JAK2 V617F Mutation}

Kuwahara H., Hiroshi F., Ken M., Mutsufusa W., Junji T., Hiroto F.

Tokyo Metropolitan Bokutoh Hospital, Japan

Background: Cerebrovascular disorders are major complications of myeloproliferative neoplasms such as polycythemia vera and essential thrombocytosis, but the precise pathomechanism is uncertain. Janus kinase 2 (JAK2), a cytoplasmic tyrosine kinase at the erythropoietin receptor, is increasingly recognized as an essential pathological molecule in these diseases, because the JAK2 V617F mutation has been frequently and specifically identified.

Methods: We retrospectively investigated cerebrovascular disorders in patients with polycythemia vera $(n=15)$ and essential thrombocytosis $(\mathrm{n}=6)$ with the JAK2 V617F mutation.

Results: The patients were 9 males and 12 females ranging in age from 34 to 82 . About half of the patients developed headache which promptly resolved after phlebotomy. Only $10 \%$ of the patients developed lacunar syndrome (cherio-oral and lateral medullary syndrome), but radiological findings revealed lacunar infarctions in $60 \%$ of the patients who underwent brain magnetic resonance imaging or computed tomography. No patients developed dementia, and leukoaraiosis was not detected in any patients. Treatment with phlebotomy, antiplatelet drugs and hydroxycarbamide prevented brain infarction.

Conclusion(s): Headache was probably triggered by blood hyperviscosity due to non-autonomous erythrocytosis. The high prevalence of lacunar infarctions was likely a manifestation of facilitated thrombogenicity under the increased shear stress at the arterioles in the brain.

\section{4}

Selenium Species and Heavy Metals in Cerebrospinal Fluid and Risk of Amyotrophic Lateral Sclerosis: A Hospital-Based Case-Control Study

Vinceti M. ${ }^{a}$, Mandrioli J. ${ }^{a}$, Bonvicini F. ${ }^{\text {, }}$ Arcolin E. ${ }^{\text {, }}$, Georgoulopoulou E. ${ }^{\mathrm{a}}$, Solovjev N. ${ }^{\mathrm{b}}$, Michalke B. ${ }^{\mathrm{b}}$

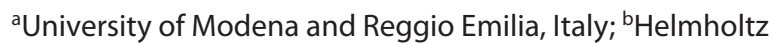
Zentrum München - German Research Center for Environmental Health, Germany

Background: Amyotrophic lateral sclerosis (ALS) is a neurodegenerative disease which has been ascribed to overexposure to selenium and some heavy metals, on the basis of epidemiologic evidence and laboratory observations. However, no data are available on the specific involvement of single selenium species, all of which have distinctive biological activities, and limited evidence has also been provided for lead, mercury and cadmium in the human.

Methods: In a hospital-admitted case-control series, we determined the Se compounds and the levels of $\mathrm{Cd}, \mathrm{Hg}$ and $\mathrm{Pb}$ in cerebrospinal fluid samples of thirty-six ALS patients and of thirty-six 
reference neurological patients. Determinations of Se compounds and of the heavy metals was performed using high pressure liquid chromatography coupled with inductively coupled plasma dynamic reaction cell - mass spectrometry according to methodologies previously established and described by one of the coauthors (B.M.).

Results: We found an excess concentration of inorganic Se forms, particularly of the hexavalent and tetravalent ones, while organic Se compounds levels were considerably decreased in ALS cases. These differences were more pronounced in older patients and in females. On the converse, no substantial differences in $\mathrm{Cd}$, $\mathrm{Hg}$ and $\mathrm{Pb}$ concentrations emerged.

Conclusions: These results suggest the occurrence of overexposure to inorganic Se species and decreased levels of Se-containing enzymes in ALS, while they offer little evidence of an involvement of three heavy metals, $\mathrm{Cd}, \mathrm{Hg}$ and $\mathrm{Pb}$, in the etiopathogenesis of this disease. However, caution must be used when inferring etiological clues from analytical results in patients affected by a severe disease such as ALS, and in hospital-referred controls. Further research on the involvement of Se in ALS etiology is clearly warranted.

\section{5 \\ Cerebrovascular Diseases Mortality Data Illustrate Improvement in Stroke Care in Poland from 1999 to 2010}

Swiat M. ${ }^{\mathrm{a}}$, Grzegorz O. ${ }^{\mathrm{a}}$, Krajewski-Siuda K. ${ }^{\mathrm{b}}$

${ }^{a}$ Medical University of Silesia, Katowice, Poland; ${ }^{b} J a g i e l l o n i a n$ University Medical College, Institute of Public Health, Poland

Background: Over the last two decades vast socioeconomic changes occurred in Poland including health care system and population aging. Stroke care system has been rapidly developing from about 2000. Cardiovascular diseases mortality started declining in Poland from early 1990s whereas cerebrovascular diseases (CVD) mortality was continuously going up at that time. CVD category constitutes stroke types of diverse etiology. To get more detailed information on recent CVD mortality trends in Poland we investigate patterns in mortality by stroke subtype from 1999 to 2010 .

Methods: We estimated age adjusted mortality rates for men and women based on death certificate data. The mortality curves were plotted for cerebrovascular diseases (CVD), cerebral infarction (CI), intracerebral hemorrhage (IH), subarachnoid hemorrhage $(\mathrm{SAH})$ and not specified stroke as haemorrhage or infarction (NSS).

Results: CVD mortality rates declined by $33 \%$ in men and by $40 \%$ in women. CI mortality remained almost unchanged but IH mortality declined by $20 \%$ in men and $38 \%$ in women. Mortality from SAH declined accordingly by $19 \%$ and $12 \%$. The most significant decline was seen in NSS mortality, $63 \%$ in men and $66 \%$ in women. NSS was recognized as a cause of death in 18280 cases in 1999 (43\% of all CVD deaths) and in 8897 cases in 2010 (25\% of all CVD deaths).
Conclusion(s): Substantial decline in CVD mortality in Poland from 1999 to 2010 was found. The number of death cases related to NSS decreased by $50 \%$ which is most likely associated with improved diagnostic standard resulting in better identification of stroke subtypes and accordingly lower decline in $\mathrm{IH}$ and $\mathrm{SAH}$ mortality and unchanged CI mortality.

\section{6}

\section{Stroke Mortality Has Finally Declined in Poland}

\author{
Swiat M. ${ }^{a}$, Grzegorz O. ${ }^{\text {a }}$ Krajewski-Siuda K. ${ }^{\mathrm{b}}$
}

${ }^{a}$ Medical University of Silesia, Katowice, Poland; ' Jagiellonian University Medical College, Institute of Public Health, Poland

Background: Stroke mortality was declining in most western and northern Europe whereas in Poland was continuously increasing in the second half of 20 century. Moreover Polish population has been continuously aging from mid 1980s what may surge stroke incidence and mortality accordingly. The aim of the study was to explore trends in mortality from stroke and cardiovascular diseases in Poland from 1976 until 2010.

Methods: Statistical data based on death certificates have been gathered from Central Statistical Office. Annual age standardized (world standard) mortality rates for cerebrovascular diseases (CVD), cardiovascular diseases (CD), ischemic heart disease (IHD) and atherosclerotic disease (ASD) from 1976 to 2010 were calculated. Men and female trends were plotted separately.

Results: Stroke mortality in Poland was increasing from 1976 until 1999 reaching 91/100 000 in men and 71/100 000 in women. Continuous decline was observed thereafter down to 60/100 000 in men and 43/100 000 in women in 2010. Similar pattern was seen for IHD but CV and ASD mortality started declining about a decade before. After switching ICD-9 to ICD-10 classification in 1997 CVD and IHD mortality rates suddenly increased but ASD mortality rate significantly dropped while gradual declining trend in CV mortality was continued.

Conclusion(s): Despite ongoing aging of the population stroke mortality started declining in Poland at last what was almost a decade behind cardiovascular disease but similar to ischemic heart disease mortality. Stroke mortality in Poland is still considerably higher then in western European countries. Death certificate data before 1997 may be biased by misclassifications of vascular death causes. 
271

\section{The Incidence of Guillain Barré Syndrome in Six Italian Regions}

D'Alessandro R. ${ }^{\mathrm{a}}$, Beghi E. ${ }^{\mathrm{b}}, \mathrm{Chi}$ A. ${ }^{\mathrm{C}}$, Filippini G. ${ }^{\mathrm{d}}$, Logroscino G. ${ }^{\mathrm{e}}$ Pugliatti M. ${ }^{f}$, Galeotti F. ${ }^{\mathrm{g}}$, Massari M. ${ }^{\mathrm{g}}$, Santuccio C. ${ }^{\mathrm{g}}$, Raschetti ${ }^{\mathrm{h}}{ }^{\mathrm{h}}$

aUniversity of Bologna, Italy; ${ }^{b}$ Istituto Farmacologico M. Negri,

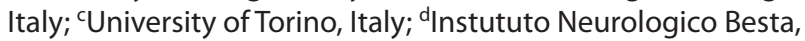

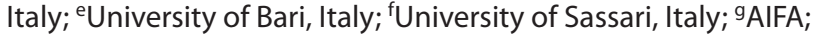
hISS

Background: The incidence of Guillain Barré Syndrome may vary from 0.16 to 3.0 per 100,000 person-years. To assess the effect of possible risk factors, carefully determined background rates are necessary. Here we present results of prospective study performed in six regions of Italy.

Methods: The six regions account for 29.5 million inhabitants (25.6 million $\geq 18$ years).

All patients $\geq 18$ years admitted to 153 neurological units for possible GBS from October 1/2010 to September 30/2011 were registered in a centralized database. Clinical and follow-up data of patients with confirmed GBS were then recorded and automatically classified according to the Brighton Criteria (BC). To assess the completeness of case collection, clinical data of patients discharged with ICD 357.0 during the study period were reviewed.

Results: Three hundred forty five patients (210 men, 135 women) were prospectively recorded through neurological units network. Among these 285 fulfilled levels 1 or 2 of the BC and nine level 3. For 51 patients the clinical-laboratory picture did not fulfil the BC.

The following forms were observed: 210 AIDP, 38 AMAN, 26 AMSAN, 24 Miller Fisher Syndrome, 47 other GBS variants. The total incidence rate was $1.4 / 100,000 /$ population. Within the six Regions the age-sex standardised incidence rates ranged from 0.80/100, 000 (in Sardinia) to 1.8/100, 000 (in Puglia).

The incidence was 1.75 among men and 1.08 among women. A clear seasonal variation was observed with a peak in January $(0.15 / 100,000)$ and the lowest rate in April $(0.05 / 100,000)$. Fiftyeight patients were further identified by reviewing the discharge codes in four regions. The whole incidence rate including these patients was 1.6/100, 000 .

Conclusion(s): The observed incidence rates are within the range observed in Europe. GBS variants may escape BC classification. A clear seasonal variation was found.

\section{4 \\ Excess Body Weight and Stroke in a New Stroke Unit in the Poorest Region in Chile}

Rivas R., Vasquez M., Daroch C.

Clinica Alemanade Temuco, Chile

Background: Stroke is the second most common specific cause of death in Chile. The risk of death from stroke in Chile is associated with age, sex and living in four specific regions of the country, including our. The increased risk in our regions is explained by the prevalence of poverty, diabetes, a sedentary lifestyle and overweight. Our objective is to assess the Excess Body Weight (EBW) in Clínica Alemana de Temuco, during January 2011 to July 2012.

Methods: We evaluated patients with a stroke diagnosis, who were evaluated in our primary Stroke Unit, were hospitalized for more than 72 hours. All patients were evaluated by two nutritionists. We obtained height and weight by a calibrated Seca scale. The study patients were divided into 3 groups according to body mass index (BMI): normal weight $(\mathrm{NM})(<25 \mathrm{~kg} / \mathrm{m} 2)$, overweight $(\mathrm{OW})(\geq 25-29.9 \mathrm{~kg} / \mathrm{m} 2)$ and obese $(\mathrm{Ob})(\geq 30 \mathrm{~kg} / \mathrm{m} 2)$.

Results: We obtained 66 patients. Mean age: 58.1 yrs (25-88), 54.5\% were men. Mean BMI: 29.1 (SD: \pm 4.35 ). NM: 33.33\%, mean BMI: 23.52 (SD: \pm 1.02), OW: 33.33\%, mean BMI: 28.08 (SD: \pm 1.64), Ob: $33.33 \%$, mean BMI: 34.12 (SD: \pm 2.61 ). EBW: $66.7 \%$. Men mean BMI: 28.67 (SD: \pm 3.49 ), Men EBW: 61.1\%. Women mean BMI: 29.53, (SD: \pm 4.45$)$.Women EBW: 70\%.

Conclusion: EBW is a preventable vascular risk factor. We can see through dietary changes and socioeconomic, an increase in excess weight. So much so that we have greater obesity and overweight than in Europe, and only similar to the U.S. According to the National Health Survey (ENS), 2010, 67\% of Chileans are in this category, however, in the National Survey of Quality of Life and Health (2012), only $33.1 \%$ claimed to be in this category. This series of case only shows that the problem is important and we should perform behaviors to lower these indicators, with much education and government guidelines.

\section{6 \\ Incidence of Transient Ischemic Attack from Ludhiana Population-Based Stroke Registry}

Sharma A.

India

Background and aims: The incidence of Transient Ischemic Attack (TIA) is unknown from developing countries. Hence we carried out this study to find out the incidence of TIA from the Ludhiana population based stroke registry.

Methods: The population of Ludhiana city is 1, 398, 467 and 935, 925 were over 18 years. The study period was from January 2011 to December 2011. All first ever TIA patients ( $\geq 18$ years) were included. TIA was defined as a focal (or at times global) neurological impairment of sudden onset, and lasting $<24$ hours. Patients data were obtained from major hospitals, scan centres and general practitioners using separate questionnaires.

Results: 1279 stroke patients were seen, and out of this 60 (4.7\%) had TIA. The mean age of TIA patients was $58.4 \pm 14.1$ years and $37(61.7 \%)$ were men. The annual incidence rate of TIA was 6.5/100, 000 (95\% confidence interval (CI): 5.3-8.4). The age adjusted incidence rate per 100, 000 was 9.3 (95\% CI: 6.9-11.6); men 10.1 (95\% CI: 6.9-13.4); women 6.9 (95\% CI: 4.1-9.8). Out of 60 patients, $24(75 \%)$ persons had TIA in carotid circulation and 8 (25\%) patients in vertebrobasilar territory. The common risk factors were: hypertension 25 (80.6\%), diabetes 15 (46.9\%), alcohol 4 $(12.5 \%)$ and desi ghee (clarified butter) intake 21 (65.6\%). 
Conclusions: The incidence of TIA was $6.5 / 100,000$ in this study. The average age of our TIA patients was lower than the reports from developed countries. Hypertension was the common risk factor.

Acknowledgement: Indian Council of Medical Research Task Force Project.

\section{Session P4: Treatment/Management}

\section{8}

\section{Subacute Administration of Zinc Induces Transcriptional Regulation of CXCL1, CXCR2 AND IGF-1 During Cerebral Hypoxia-Ischemia in the Rat}

Leon-Chavez B.A. ${ }^{a}$, Blanco-Alvarez V.M. ${ }^{a}$, Soto-Rodriguez G. ${ }^{\text {, }}$ Gonzalez-Barrios J.A. ${ }^{\mathrm{b}}$, Martinez-Fong D. ', Piña-Leyva C. a, Ugarte A. ${ }^{a}$ Eguibar J.R. ${ }^{\text {a }}$

aBenemerita Universidad Autonoma de Puebla, Mexico;

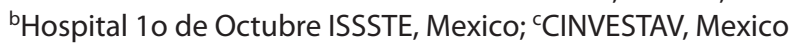

Background: In the cerebral ischemia increases the expression of chemokines RANTES, MCP-1 and CXCL1. In the brain, these chemokines promote leukocyte migration and participate in the proliferation of astrocytes and oligodendrocytes. One of the therapeutic strategies consists of zinc administration to decrease the cell death and infarct volume following brain ischemia. The aim of this work was to evaluate the effect of $\mathrm{Zn}$ subacute administration on the transcriptional regulation of IGF1, CCL2, CCL5, CXCL1 and their CCR2, CCR5 and CXCR2 receptors after a transient common carotid artery occlusion (CCAO).

Methods: Male Wistar rats were separated into two groups; one group underwent the CCAO for $10 \mathrm{~min}$., and the other group was treated with $\mathrm{ZnCl} 2$ (2.5 mg/kg i.p.) every 24 hours during 4 days before the CCAO. The temporo-parietal cortex was dissected out at different times $(4,8,12,24,36,96$, and $168 \mathrm{~h}$ post-reperfusion). Without treated rats were used as control.

Results: Our results showed two decreases in the level of mRNA for IGF- 1 at $4 \mathrm{~h}$ and at the $24 \mathrm{~h}$ post-reperfusion in the group of rats with ischemia. The subacute administration of $\mathrm{Zn}$ before CCAO increased the levels of IGF-1 mRNA at 8 hours and at $96 \mathrm{~h}$, of CXCL1 at $4 \mathrm{~h}$ post-reperfusion, in addition, CXCL1 mRNA decreased at $24 \mathrm{~h}$. CCR5 decreased at $24 \mathrm{~h}$ and CCR2 increased and CXCR2 mRNA increased at $8 \mathrm{~h}$ post-ischemia and CCL2 decreased at $8 \mathrm{~h}$.

Conclusion(s): Our results suggest that the subacute administration of zinc before CCAO modulates the neuroinflammation process by downregulation of CCR5 $\mathrm{mRNA}$ and up-regulation of CXCR2 and CCR2 mRNAs, besides of exercising a neuroregeneration effect through the up-regulation of CXCL1 and IGF-1 mRNA in the hypoxic-ischemic zone.
50

Population-Based Study on Long-Term Outcome and Caregiver Burden in Stroke

Winter Y., Nater U., Oertel W., Dodel R.

Philipps-University Marburg, Germany

Background: Studies on long-term clinical and neuropsychological outcome in stroke performed using epidemiologic design are limited. Recently, we initiated a population-based study on long-term outcome and caregiver burden in ischemic stroke.

Methods: The population-based study was started in 3/2012 in the district Marburg-Biedenkopf (reference population 160.000 inhabitants) Hessia, Germany and will stop in 2/2013. Inclusion criterion is an acute ischemic stroke. The follow-ups are performed at 6 and 12 months after stroke. Long-term outcome is evaluated using a multidimensional approach including assessment of disease severity (NIH Stroke Scale), depression (Geriatric Depression Scale), neuropsychological impairment (Mini-Mental State Examination, Vascular Dementia Assessment Scale-cog), health-related quality of life (Stroke Specific Quality of Life Scale, EuroQol) and caregiver burden (Caregiver Strain Index, Life Satisfaction Questionnaire).

Results: Preliminary results $(\mathrm{n}=150)$ of ongoing study show a high prevalence of depression in stroke patients (up to $40 \%$ ). Approximately $30 \%$ of patients develop cognitive impairment or experience a worsening of pre-existing cognitive deficits. Quality of life was considerably reduced due to stroke and its complications. Further preliminary results including factors influencing the stroke outcome will be reported.

Conclusions: We present a large-scale ongoing study using a population-based design to evaluate long-term outcome after stroke. Multidimensional approach in assessment of stroke outcome and caregiver burden will provide the most comprehensive data on the course of cerebrovascular disease and will help to reveal modifiable determinants of outcome.

\section{3}

\section{Nutritional Support Could Prevent Protein Energy} Malnutrition in Stroke Patients

Djaja N.

Atma Jaya University, Indonesia

Background: Several studies showed that Protein Energy Malnutrition, was almost always found in stroke patients either during their stay in the hospital or after they were discharged. Soybean cake contains vegetable protein with adequate essential amino acids content, easy to digest, and is expected to be able to prevent malnutrition in combination with albumin for stroke patients.

The aim of this study is to find out whether early supplementation of soybean cake and albumin formula could prevent PEM by measuring the pre albumin level before and after administration of the formula. This is used as nutritional status indicator. 
Methods: There are 22 study subjects, the respondent must be 40 years or older, they didn't get albumin nor other nutrition parenterally besides fluid and electrolytes, and all had normal liver and renal function. These subjects received soybean cake and albumin formula intervention through nasogastric tube for 4 days.

Results: Pre intervention pre albumin level average was 15, $2 \mathrm{mg} / \mathrm{dL}$, and after 4 days of nutritional intervention, there was noted an increased in pre albumin level average was $1,8 \mathrm{mg} / \mathrm{dL}$. Bivariant $\mathrm{T}$ Test statistic analysis showed significant difference toward the rise between pre and post nutrition intervention.

Conclusion: Early nutritional support using soybean cake and albumin formula could increase pre albumin serum level within few days after stroke attack.

\section{9 \\ Does Aggressive Care for Patients with Traumatic Brain Injury Alter 30-Day Mortality?}

\section{Mirek S. ${ }^{a}$, Opprecht N. ${ }^{\mathrm{a}}$, Bonithon-Kopp C. ${ }^{\mathrm{b}}$, Freysz M. ${ }^{\mathrm{a}}$ \\ ${ }^{a} \mathrm{CHU}$ Dijon, France; ' INSERM CIE01, CIC-EC, France}

Background: Intracranial pressure (ICP) monitoring of traumatic brain Injury (TBI) possesses indications defined by the ANAES 1999 guidelines1.

The purpose of this work, conducted on the data of the FIRST study2, was to compare the D 30 mortality of Glasgow score $(\mathrm{GCS})<8$ patients ICP monitored or not.

Methods: The FIRST study produced pre- and intra-hospital epidemiological data for patients with severe closed-head trauma who benefited from ICP monitoring during their stay in one of the study's 14 French University Hospitals. The study population was comprised of all patients with a GCS $<8$.

Data such as age, gender, type of accident, systolic arterial pressure, GCS, SpO2, ISS (Injury Severity Score), head AIS (Abbreviated Injury Score), mydriasis and surgery were analyzed in a univariate manner, then introduced into a multivariate model of logistic regression. A threshold of $\mathrm{p}<0.05$ was deemed to be significant.

The variable of interest was the aggressiveness of care provided by the specialized university hospital center, defined by ICP monitoring for more than $50 \%$ of patients with a GCS $<8$. The main outcome measure was mortality at D30.

Results: Of the 3205 patients in the FIRST database, 931 met the inclusion criteria with 363 patients in the aggressive center group and 568 in the non-aggressive group.

Overall, the mean age was of 40 years, the M/F gender ratio of $3 / 1$ and the median ISS of 29 . ICP was monitored in $58 \%$ of patients in the aggressive center group vs. $23 \%$ in the non-aggressive center group $(\mathrm{p}<0.001)$, the AIS was $>3$ in $79 \%$ of patients vs. $73 \%$ (ns). D3 mortality was of $18 \%$ vs. $28 \%$ (p < 0.001 ); D30 mortality was of $34 \%$ vs. $40 \%(p=0.06)$ in these two groups respectively.

After adjustment for various factors, we did not find any significant difference in mortality at D3 (OR 0.76, CI 95\% 0.45 -1.26). There was no significant overall difference in D30 mortality asso-

Poster Presentations ciated with center aggressiveness (OR $=0.99$, CI95\% 0.65-1.52). There was, however, significant interaction between center and age $(\mathrm{p}=0.02)$. In patients over the age of 55 years, center aggressiveness was associated with a reduction in D30 mortality of nearly $60 \%$ ( $\mathrm{OR}=0.41$, CI95\% $0.18-0.97)$, whereas in patients under the age of 55 years, no such association was observed (OR = 1.33 , CI95\% 0.82-2.17).

Conclusion(s): The management of TBI appears to highly heterogeneous between the different specialized care university centers, despite the existence of clearly defined guidelines, particularly for intracranial pressure monitoring. Aggressive care is associated with a reduction in delayed D30 mortality in patients over the age of 55 years, though with no impact on early D3 mortality. Even if the data resulting from this study do not allow us to compare subsequent survival quality, these results obtained in University Hospital units would tend to argue in favor of an updating of guidelines. This could spur the standardization of patient care.

References: 1. Ann Fr Anesth Reanim. 1999;18:11-159.2. Crit Care. 2011;15(1):R34.

\section{9 \\ Implementing a Multi-Drug Registry Within the
Observatoire Français de la Sclérose en Plaques}

Vukusic S. ${ }^{\mathrm{a}}$, Clanet M. ${ }^{\mathrm{b}}$, Confavreux C. ${ }^{\mathrm{a}}$

aHôpital Neurologique et Fondation EDMUS pour la Sclérose en Plaques, Toulouse, France; b Hôpital Purpan, Toulouse, France

The arrival of new drugs for multiple sclerosis (MS) means a potential multiplication of post-marketing studies. How to organize those studies without multiplying protocols and organizations? How to deal with the necessary switches of patients from one study to another? How to provide long term data when considering serious adverse events (SAEs) like cancers? How to estimate the potential of cumulative risks when using all those drugs consecutively in a same patient, which is a more than probable situation for a disease that encompasses several decades? How to deal with these new obligations without increasing the burden of work for neurologists?

The Observatoire Français de la Sclérose En Plaques (OFSEP) is a national cohort of currently more than 36.000 patients in France that has been selected in January 2011 for a ten-year tenure as part of the "Investments for the Future" programme of the Présidence de la République Française. Through systematic longitudinal follow-up and combination of clinical data with biological samples and neuro-images, the OFSEP project aims at improving the quality, efficacy and safety of patients' care and at fostering clinical, basic and translational research in MS.

A general effort for mutualizing human, financial, methodological and technical means in a vast and unique phase IV study or register could answer the requirements of each individual postmarketing study on safety, efficacy, good use and risk/benefit balance of drugs used for the treatment of MS. This observational study could be run in daily practice, on the long-term, in a large set of patients, with control groups of untreated patients followed simultaneously. Therefore, the OFSEP cohort is the perfect tool, ready to host the first national multi-drug registry for MS. 


\section{4}

\section{Clinical Factors are Related to Early Non-Response After Intravenous tPA}

Apoil M. ${ }^{a}$, Turc G. ${ }^{a}$, Tataru A.M. ${ }^{a}$, Allibert R. ${ }^{a}$, Meder J.-F. ${ }^{a}$, Mas J.-L. ${ }^{b}$, Baron J.C. ${ }^{a}$, Oppenheim C. ${ }^{\text {, }}$ Touze E. ${ }^{\mathrm{a}}$

aUniversité Paris Descartes Sorbonne Paris Cité, Centre de psychiatrie et de neurosciences, INSERM UMR S894, Hôpital Sainte Anne, Paris, France; ${ }^{b}$ Centre Hospitalier Sainte-Anne, France

Background: Little studies have focused on potential predictors of no clinical improvement 1-hour post intravenous (IV) rt-PA for acute stroke. However, the identification of early nonresponders should be helpful for the selection of the best candidates for additional endovascular treatment.

Objective: To assess simple patients' characteristics associated with early non-response to IV rt-PA.

Methods: Baseline clinical and radiological data of 269 consecutive patients thrombolysed $\leq 4.5 \mathrm{hrs}$ after stroke onset between January 2003 and June 2012 in our center were collected. Brain MRI was performed in 246 (91\%) patients. The absence of very early neurological improvement (VENI) was defined by $\leq 40 \%$ decrease in NIHSS score 1 hour after IV rt-PA. Odds ratios (OR) and $95 \%$ confidence intervals $(95 \% \mathrm{CI})$ were estimated using binary logistic regression.

Results: Among the 269 patients included (mean \pm sd age: $67.7 \pm 14.8$, 56\% male, median(IQR) baseline NIHSS: $14(8-19)$, onset-to-treatment time(OTT)(min): 160(128-195)), $75 \%$ patients had no VENI at 1 hour. In multivariate analysis, the absence of VENI was associated with no favorable 3-month outcome $(\mathrm{OR}=2.82 ; 95 \% \mathrm{CI}, 1.26-6.34 ; \mathrm{p}=0.01)$. Baseline NIHSS $(\mathrm{OR}=1.10 ; 1.04-1.16$ per 1 -point increase, $\mathrm{p}=0.001)$, and OTT $(\mathrm{OR}=1.12 ; 1.63-7.21$ per $10 \mathrm{~min}, \mathrm{p}=0.001)$ were associated with non-response at 1 hour.

Conclusion(s): Non-response 1 hour after IV rt-PA is strongly associated to poor 3-month outcome. Lack of VENI is associated with baseline NIHSS and OTT. There is a need to identify other very early radiological or biological predictors for non-response to rt-PA that could help selecting patients requiring additional reperfusion therapies.

\section{7}

\section{Does the Response to Thrombolysis with rt-PA} Depend on Thrombus Composition?

\section{Allibert $R$.}

Université Paris Descartes Sorbonne Paris Cité, Centre de Psychiatrie et de Neurosciences, INSERM UMR S894, Hôpital Sainte Anne, Paris, France

Background: It has been suggested that efficacy of intravenous (iv) tPA in ischemic stroke could be related to thrombus composition, but data are limited. We therefore assessed whether the early clinical response to iv-tPA was related to ischemic stroke etiology.
Methods: Baseline clinical and radiological data of 269 consecutive patients thrombolysed $\leq 4.5 \mathrm{hrs}$ after stroke onset between $01 / 2003$ and $06 / 2012$ were collected. Only patients who had MRI (91\%) were included. Very early neurologic improvement (VENI) was defined by a $40 \%$ improvement of NIHSS one hour after end of treatment. Recanalization was assessed on MR-angiography at 24 hours. Extensive etiological workup was done in all patients. Stroke subtype was assessed using ASCO score and was available for 184 patients at the time of our analysis.

Results: Compared to patients with atherosclerotic stroke $(\mathrm{n}=$ $21)$, those with definite cardioembolic stroke $(n=63)$ were non significantly more likely to have VENI ( $32 \%$ vs. $14 \%$; $\mathrm{p}=0.13$ ). After adjustment for age, admission NIHSS, time to thrombolysis and occlusion site, OR was 3.3 (95\%CI; 0.8-14.0, p = 0.11). After inclusion of patients with cryptogenic stroke $(A=0 ; S=0 ; C \neq 1 ; O$ $=0)(n=21)$ in the cardioembolic group, results were unchanged (adjusted $\mathrm{OR}=3.2 ; 0.8-12.6, \mathrm{p}=0.10$ ). There was no association between stroke subtype and recanalization at 24 hours.

Conclusion(s): Despite a lack of statistical power, our findings support the hypothesis that efficacy of iv-tPA depends on thrombus composition. This results need to be confirmed on a larger sample and using an additional early recanalization outcome.

\section{9 \\ Low Protein Diet Could Prevent Akut Kidney Injury in Stroke Patients}

Djaja $N$.

Atma Jaya University, Indonesia

Background: Stroke is the leading cause of death in every hospital in Indonesia. The death rate of newly formed or recurrent stroke is estimated around 750.000 case every year nation wide, 200.000 of which are recurrent stroke. Stroke patients have higher risk to develop another stroke attack. In 5 years time, the recurrence of stroke attack is estimated around $30-43 \%$. In many cases, elderly stroke patients who were admitted to the hospital with recurrent stroke attack also suffer from anorexia which leads to hypoalbuminemia, hyponatremia, hypokalemia and impaired Renal function marked by a rise in ureum level without elevation in blood creatinin levels.

Methods: This study uses pre and post nutrition intervention method. Hospital's low protein diet formula was given through nasogastric tube. The amount of calorie was adjusted according to basal needs $\mathrm{x} 1,4$ and consisted of carbohydrate, protein 0,8 gram $/ \mathrm{kg} \mathrm{BB}$ (only albumin protein) and $25 \%$ fat. This formula was given to 44 stroke patients (mean age of patients was $40 \pm 80$ years) who had been admitted to Atma Jaya Hospital for at least 7 days and met the inclusion \& exclusion criteria, without history of renal failure. All the patients' intake and fluid balance were monitor.The average albumin level of these patients was $+3 \mathrm{mg} / \mathrm{dL}$.

Results: After receiving nutrition (NGT) and fluid (parenteral nutrition) intervention during 7 day, the result is as follows: $82 \%$ patients have reduction of ureum and creatinin level significant, increase in Natrium level and Kalium level.

Conclusion(s): When there was inadequate calorie intake, protein from muscle might be broken down marked by a rise in blood 
ureum level with or without an increase in creatinin level. In this condition, electrolyte level, such as Natrium and Kalium, could be corrected with appropriate nutritional support (adequate calorie, low protein albumin and mineral) and therefore prevents acute kidney injury in elderly patients with anorexia.

\section{4}

Cardiac Autonomic Balance in Children with Epilepsy, Value of Antiepileptic Drugs

Omneya I.Y., Elrashidy O., Shatla R.

Faculty of Medicine Ainshams University, Egypt

Background: Antiepileptic drugs (AEDs) have various cardiac complications. However, they might prevent SUDEP by improving seizure control. HRV is a useful tool to assess cardiac autonomic balance.

Aim: Evaluate cardiac autonomic status in children with epilepsy on AEDS (this section outlinesimportance, objectives/aims or hypothesis of the study).

Methods: 60 children with epilepsy were divided into: group I: newly diagnosed patients not receiving AEDs (50\%) and group II: epileptic patients on regular AEDs further subdivided into: II-a receiving mono-therapy $(26.7 \%)$ and II-b receiving poly-therapy (23.3\%). Forty age and sex-matched healthy children served as controls. patients were subjected to video-recorded EEG, Holter ECG for time and frequency domains of HRV, and standard ECG recording for QTc, QTd, (in this section, design, subjects, and statistical analysis used in the study are briefly described).

Results: Mean values of all time domain, TP and HF were significantly lower, LF and LF//HF, QTc and QTd were significantly higher in group I than IIand in patients than controls. No significant difference was found between patients on different AEDs regimen regarding HRV values.. mean values of QTc, and QTd were insignificantly higher in EEG abnormality G-II. Significant Negative correlation was found between Chalfont severity score and patients pNN50 (in this section, main results of the study are presented as clearly as possible).

Conclusion(s): Children with epilepsy have cardiac autonomic dysfunction shown in their HRV assessment. Patients on AEDS had better autonomic balance than those on no AEDS. Holter and ECG follow up of should be considered for early detection of those at high-risk cardiac complications (in this section, importance of results of the study for practice and/or future research is briefly described).

\section{1}

\section{A Novel Injectable Chitosan Sponge to Enhance Remyelination Post Spinal Cord Injuries}

Mekhail M.

McGill University, Montréal, Canada

Background: A contusion spinal cord injury (SCI) occurs when the spinal cord is exposed to a severe mechanical impact. One of the consequences of SCI that take place early in the cascading secondary injury is the demyelination of intact axons due to the death of oligodendrocytes through necrosis or apoptosis. Remyelination that takes place post injury is usually abnormal; the myelin produced is thinner and have shorter internode segments. Therefore, enhancing remyelination, by increasing the number of remyelinated axons, could result in recovery of neurological function that could be lost otherwise. The research project thus involves the use of a combinatorial approach (injectable sponge + drug delivery mechanism) to develop a therapeutic measure that can be administered shortly after a SCI to prevent neuronal loss due to demyelination. The objectives of the research project are to: (1) Develop and characterize the physical and chemical properties of the injectable sponge as well as its biocompatibility; (2) Study Oligodendrocyte Progenitor Cells' (OPg) behaviour on the novel sponge; (3) Fabricate Poly(lysine) nanoparticles, with and without a lipid bilayer coating to deliver a slow release of Neurotrophin 3 (NT-3) and a burst release of Platelet-Derived Growth Factor (PDGF) respectively; (4) Study the effect of the sponge/nanoparticles on OPgs' proliferation and differentiation in-vitro; (5) Inject the chitosan sponge in a contused SCI mouse model and study remyelination in-situ.

Methods: A novel injectable chitosan sponge was developed that rapidly solidifies in-situ. Solidification in-situ occurs upon mixing a chitosan solution with a solution containing a novel anionic crosslinker (molecule cannot be identified due to pending patent application). The anionic molecule used to crosslink the chitosan has also been previously shown to enhance remyelination in-situ after a SCI. Therefore, the anionic molecule serves two purposes, crosslinking and enhancing remyelination. Four combinations were developed to provide varying sponge properties that could be tested at the site of injury. One way ANOVA Tukey Tests were carried out to measure significant differences amongst multiple experimental groups.

Results: The chitosan sponge has been shown to form rapidly after mixing the chitosan and anionic crosslinker solutions. All sponges formed in less than 1.6 seconds (as measured by an Impedance Analyzer) and SEM revealed a highly porous sponge with excellent pore interconnectivity. Sponges retained water up to ten times their weight and were shown to be much softer mechanically compared to the spinal cord. Biocompatibility studies using fibroblasts demonstrated excellent attachment and proliferation on all four sponges for up to 7 days. All results are indicative of a promising novel injectable biomaterial that could be used for enhancing remyelination post SCI.

Conclusion(s): Currently the behaviour of OPgs on the injectable chitosan sponges is being studied and nanoparticles containing NT-3 and PDGF are being developed. Findings from this research could, in the long run, result in the development of a new therapeutic modality that could lessen neurological losses after a SCI and improve the quality of life of patients. 


\section{6}

\section{The Chronic Pain Association with Reduction in Cognitive Functioning: An Exploratory Study}

Nadar M., Jasem Z.

Kuwait University, Kuwait

Background: chronic pain has been associated with reduction in cognitive skills. However, not all cognitive skills are affected and there is conflicting evidences among studies on the magnitude of cognitive effects. The purpose of the study was to examine the relationship between presence of chronic pain and decline in specific cognitive functions and if it is negatively correlated with the severity of pain or its duration.

Methods: Sixty-nine participants were assigned into two agematched groups; clients with chronic pain compared to healthy controls. The participants completed tests measuring pain and cognition. Pain assessments included its type, cause, severity, and duration of pain. the cognitive processes assessments included immediate and delayed recall (Contextual Memory Test), short term memory (Digit Forward Test), working memory (Digit Backward Test), processing speed and alternating attention (Trail Making Test), concentration and selective attention (d2 Test), and executive function (A Quick Test). one-way ANOVA and partial correlation were used to analyze the data.

Results: The chronic pain group had significantly lower performance than the control group in four cognitive measures; concentration $(\mathrm{p}=0.003, \mathrm{~F}=9.870)$, selective attention $(\mathrm{p}<0.000, \mathrm{~F}=$ $13.433)$, delayed recall $(\mathrm{p}=0.014, \mathrm{~F}=6.353)$, and executive function - naming subtest $(\mathrm{p}=0.029, \mathrm{~F}=4.980)$. Severity and duration of pain were not correlated with variations in cognitive performance.

Conclusion(s): Clients experiencing chronic pain showed decline in some cognitive skills, although the decline was not negatively correlated with the severity of pain or its duration. In view of this, targeting cognition is important to reduce the decline, even when the problem is not related to cognition.

\section{5 \\ Efficacy of Different Ketogenic Diet Modalities in Childhood Intractable Epilepsy}

Abdel-Hamid I.A., Gabr S., Shaaban S., Shatla R.H., El-Rashidy O., Nassar M.

Faculty of Medicine, Ainshams University, Egypt

Background: Considering that approximately $30-40 \%$ of patients with epilepsy are refractory to anticonvulsants and many of them are not surgical candidates, there is a need for further therapies.

Objectives: The study was designed to evaluate the efficacy, safety and tolerability of the ketogenic diet (KD) whether classic 4:1 formula or the modified Atkins diet (MAD) and their effect on the anthropometric measurements and lipid profile in intractable childhood epilepsy.
Patients and methods: Anthropometric measurements and serum lipid profile were measured upon enrollment and after 3 and 6 months in 40 patients with symptomatic intractable epilepsy. Fifteen were given MAD diet, ten were kept on classic 4:1 ketogenic liquid formula and the rest were allowed to eat as desired.

Results: Both types of KD were safe and tolerated by all except 4 patients. The anthropometric measurements showed no significant differences between the patients who received KD and those who didn`t whether initially or during the follow up. The liquid ketogenic formula group showed significantly better body mass index compared to those who didn 't receive KD after 6 months. The lipid profile of KD patients was within normal limits for age and sex during the study period. The rate of change of frequency and severity of seizures showed best improvement in ketogenic liquid formula patients followed by the MAD group then the patients on anti-epileptic medications alone.

Conclusion: The KD, whether classic 4:1 or MAD is a tolerable, safe and effective adjuvant therapy for intractable symptomatic childhood epilepsy with limited adverse effects on the growth parameters and accepted changes in the lipid profile. The liquid ketogenic formula patients showed better growth pattern and significantly more seizure control than MAD ones, importance of results of the study for practice and/or future research is briefly described).

\section{Session P5: Neurosurgery}

216

Atypical Thunderclap Headache

Holleran K., Rasiah S.

St Vincent's Hospital, Melbourne, Australia

\section{Case Report:}

Several months of fatigue, muscular aches and pains and influenza-like symptoms preceded diagnosis of a heart murmur in our previously fit and well 48 year old patient. After admission for empirical treatment of infective endocarditis, our patient suffered the classical "thunderclap" headache and was transferred to our neurosurgical unit for management of subarachnoid haemorrhage. Multiple imaging modalities revealed numerous 'mycotic' or infective aneurysms - of the left posterior cerebral artery, ulnar artery, hepatic artery and splenic hilum. Following $16 \mathrm{~S}$ rDNA sequence analysis, Rothia aeria was isolated from blood cultures. This is the first known case in the English language literature of a patient with multiple aneurysms and infective endocarditis related to R. aeria.

Preliminary results indicated that home visits by psychiatrically trained and experienced nurses were the single most effective factor with influence on the outcome on depressive symptoms in elderly patients. ADM was of lesser importance. Side effects were in the doses used here of insignificant importance. This study indicates that a larger study with double-blind design of a clinical trial with placebo arm should be conducted. 
Session P6: Neurorehabilitation

69

Post-Stroke Disposition and Associated Factors in a Population-Based Study. The Dijon Stroke Registry

Béjot Y.a , Troisgros O. ${ }^{\mathrm{a}}$, Gremeaux V. ${ }^{\mathrm{b}}$, Benaïm C. ${ }^{\mathrm{b}}$, Casillas J.M. ${ }^{\mathrm{b}}$, Giroud M. ${ }^{\mathrm{a}}$

aRegistre dijonnais des AVC, EA 4184, IFR 100 Santé-STIC, France; ' University Hospital of Dijon, France

Background: The organization of post-stroke care will be a major challenge in coming years. We aimed to assess hospital disposition after stroke and its associated factors in clinical practice.

Methods: All cases of stroke from 2006 to 2010 were identified from the population-based Stroke Registry of Dijon, France. Demographic features, risk factors, and pre-stroke treatments were recorded. Admission stroke severity was assessed using the National Institutes of Health Stroke Scale (NIHSS) score. At discharge, we collected dementia, disability using the modified Rankin scale (MRS), length of stay (LoS), and hospital disposition (home, rehabilitation, convalescent home, and nursing home). Multivariate analyses were performed using logistic regression models to identify associated factors of post-discharge disposition.

Results: Of the 1069 stroke patients included, 913 survived acute care. Among them, 433 (47.4\%) returned home whereas 206 (22.6\%) were discharged to rehabilitation, $134(14.7 \%)$ were admitted to a convalescent home and 140 (15.3\%) to a nursing home. Old patients, those under anticoagulants before stroke, those with either severe stroke on admission, severe disability at discharge, dementia, or prolonged LoS were less likely to return home. Moreover, advanced age, severe initial stroke, severe disability at discharge, and dementia were associated with admission to convalescent and nursing homes rather than rehabilitation centres.

Conclusion(s): This population-based study demonstrated that post-discharge destinations are associated with several factors. Our findings may be useful to establish health policy concerning the organization of post- stroke care.
152

\section{A Comparison of EEG Biofeedback and Classical Rehabilitation on Motor Skills of Children with Mild Central Motor Disorders and ADHD/ADD}

Ziakova E. ${ }^{\text {, }}$ Bartko D. ${ }^{\mathrm{b}},{ }$ Klobucka S. ${ }^{\mathrm{c}}$

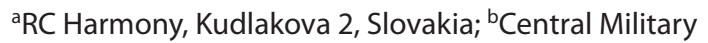
University Hospital, Slovakia; 'Rehabilitation Centre Harmony, Slovakia

Background: Medical rehabilitation helps the patients to reach physical, psychological, social potential. New, robotic aided rehabilitation has advantages.Aim of study was to compare the effect of EEG biofeedback with classical neurorehabilitation.

Methods: Material consisted of 60 children with mild central motor disorders with ADHD/ADD, divided in two subgroups:1st:30 children(mean age of 8.9), they underwent EEG biofeedback.2nd:30 children (mean age of 8.5) they underwent classic neurorehabilitation. Neurorehabilitation in both groups consisted of 30 sessions, 2-3 times/week/30-45 minutes. PANESS/ CIT tests(assessing motor ability, impulsivity, attention) before and after procedures.

Results: Our study showed different changes in gaits/hopping/ station in neurofeedback and classical neurorehabilitation:The mean achieved score of neurofeedback after finishing procedure was lower $(\mathrm{M}=25)$ comparing to score before procedure $(\mathrm{M}=$ 58.57), the mean achieved score of classical rehabilitation after finishing $(M=45.83)$ was not changed significantly(PANESS test). Wilcoxon Signed Ranks Test of gaits/hopping/station showed statistically significant better score of EEG biofeedback comparing to classical neurorehabilitation $(\mathrm{Mdn}=55,00, \mathrm{p}<0.01, \mathrm{Z}=-4,78, \mathrm{r}=$ -0.62 ). Analysis provides evidence that neurofeedback is significantly more effective comparing to classical neurorehabilitation in followed parameters.

Regarding CIT, the results showed that mean achieved age of impulsivity after neurofeedback was higher $(\mathrm{M}=9.57)$ comparing to achieved age before therapy $(M=7.73)$ and mean achieved age of attention after procedure was higher $(M=197.0)$ comparing to achieved age before procedure $(M=9.23)$. Changes in overall time in EEG neuro feedback after procedure showed that EEG neurofeedback achieved lower overall time ( $\mathrm{M}=95.02)$, comparing before procedure $(\mathrm{M}=111.78)$. The same parameters in classical rehabilitation after procedure showed lower overall time $(\mathrm{M}=$ $110.58, \mathrm{SD}=21,93)$ comparing to before therapy $(M=132.79, \mathrm{SD}$ $=23.57)$. Both procedures documented an improvement in overall time but absolute values are better in neurofeedback group.

Conclusion(s): Our study documents that EEG neurofeedback is significantly more effective in motor and neuropsychological deficit in selected group of children with mild to moderate motor and neuropsychological deficit.

Supported by EU grant ITMS2622022009. 


\section{1}

Improvement of Motor Functions in Patients with Bilateral Spastic Cerebral Palsy After Robotic Assisted Treadmill Training (RATT): The Effect of Number of Therapeutic Units

Klobucka S. ${ }^{\mathrm{a}}$, Ziakova E. ${ }^{\mathrm{a}}$, Kovac M. ${ }^{\mathrm{b}}$

${ }^{a}$ Rehabilitation Centre Harmony, Slovakia; ${ }^{b}$ Clinic of Neurology, Faculty Hospital Nove Zamky, Slovakia

Background: The aim of the study was to assess the impact of number of RATT sessions on improvement of gait and motor functions in patients with cerebral palsy (CP).

Methods: 22 children ( 8 males and 14 females) with bilateral spastic CP, aged 4, 5-25, 1 years underwent 40 therapeutic units (T.U.) of RATT using driven gait orthosis Lokomat with a frequency of 3 to 5 times a week. Before and after therapy the patients were examined through a range of Gross Motor Function Measure (GMFM-88), dimensions A (lying, rolling) B (sitting), C (crawling, kneeling), D (standing) and $\mathrm{E}$ (walking, running, jumping), 6-minute walking test (6minWT), 10 - metre walk test (10 MWT), Functional Ambulantion Categories (FAC), GMFCS (Gross Motor Function Classification System).

Results: To evaluate the results, we used Wilcoxon signedrank test. Patients demonstrated highly statistically significant improvement $(\mathrm{p}<0,001)$ in all dimensions of the GMFM after completing 20 and then the 40 sessions. We also observed a highly statistically significant increase $(\mathrm{p}<0,001)$ in walking speed, endurance when walking, and rating scales FAC, GMFCS. Comparing the average improvement (\%) in outcome parameters after 20 T.U. and after 40 T. U., we documented a statistically highly significant difference $(\mathrm{p}<0,001)$ in the dimension of $A, B$, $\mathrm{C}, \mathrm{D}$ and overall improvement in the GMFM and a statistically significant difference $(\mathrm{p}<0,05)$ in GMFM dimension E test, 10 MWT, 6 minWT.

Conclusions: After completing of 40 sessions, patients have improved more significantly than after 20 completed sessions. Our study indicates, that the number of therapeutic units of RATT affects the amount of the achieved improvement.

\section{5 \\ Transcutaneous Neuromuscular Electrical Stimulation (NMES) Is More Effective Than Traditional Therapy (TT) for Dysphagia Rehabilitation: A Meta- Analysis}

Lifen C., Tan C.

The Second Affiliated Hospital of Chongqing Medical University, China

Background: Dysphagia is a social health problem. Because of its high prevalence and its dramatic consequences, The prevalence of dysphagia among individuals over 50 years of age is estimated to range from 16 to $22 \%$. It is estimated that $20-40 \%$ of patients have feeding difficulties among special populations such as those with head injuries, cerebrovascular accidents, and Parkinson's disease. There is still debate over whether the effect of transcutaneous neuromuscular electrical stimulation (NMES) on dysphagia rehabilitation is superior than traditional therapy (TT). The purpose of this meta-analysis was to assess the overall efficacy by comparing the two treatment protocols.

Methods: Data sources: Published studies in the English language medical literature were obtained by comprehensive search (January 1966 to December 2011) of Medline, Cochrane, and EMBASE databases.

Study selection: Studies that compared the efficacy of treatment with NMES vs. TT in dysphagia rehabilitation and that clinical outcomes were ascertained were assessed.

Data extraction: Two reviewers independently performed data extraction. Data for assessment of swallowing function improvement and airway protection were extracted as scores of Swallowing Function Scale and penetration-aspiration scale (PAS) change from baseline (change scores) and pooled using a fixed effect model.

Statistical analysis: Meta-analysis was performed using Review Manager Software 5.0 (Cochrane Collaboration, Oxford, United Kingdom), according to "2009 Updated Method Guidelines for Systematic Reviews in the Cochrane Back Review Group" and "Cochrane Handbook for SystematicReviews of Interventions 5.0.2". Statistical analysis for continuous variables was carried out. Heterogeneity was measured using the I-squared (I2) and Chisquare (x2)-test. $\mathrm{P}<0.05$ was considered to indicate statistical significance.

Results: Six studies including 192 patients were analyzed. The change scores in swallowing function scales of NMES in patients with dysphagia was significantly higher compared with TT (standardized mean difference $[\mathrm{SMD}]=0.5,95 \%$ confidence interval $[\mathrm{CI}]: 0.20$ to $0.80, \mathrm{P}=0.001)$. However, there were no differences between NMES and TT in dysphagia post stroke $(\mathrm{SMD}=0.32$, 95\% CI:- 0.13 to $0.78, \mathrm{P}=0.16$ ). The change scores in PAS was significantly higher in MNES than those in TT (WMD $=1.7,95 \%$ CI:0.52to $2.87, \mathrm{P}=0.005)$, but the result is not stable when we conduct sensitivity analysis. There was no significant heterogeneity in the comparisons.

(in this section, main results of the study are presented as clearly as possible)

Conclusion(s): Transcutaneous neuromuscular electrical stimulation is more effective for treatment of adult dysphagia patients of variable etiologies than traditional therapy. However, in patients with dysphagia post stroke the effectiveness was comparable.

173

Associations of Physical Activity, Allodynia and Pain in Type 2 Diabetics with Painfull Peripheral Neuropathy

Demirtas Raziye N., Kebapci N., Efe B.

Eskisehir Osmangazi University, School of Medicine, Turkey

Background: The purpose of this study was to assess the correlations between physical activity, allodynia and pain in a sample 
of people with diabetes mellitus (DM) and painfull peripheral neuropathy (PPN).

Methods: Diagnosis of DM was established according to WHO criteria. Twenty people with DM and PPN (5 men and 15 women, mean age $=58,25$ years $[\mathrm{SD}=12.08]$, and mean body mass index $=29,78 \mathrm{~kg} / \mathrm{m}(2)[\mathrm{SD}=6.23]$ ) participated in this study. The Six-Minute Walk Test (SMWT) distance and the physical mobility (PM) domain of the Nottingham Health Profile were used to assess the physical activity.. Allodynia was identified with Visual Anolog Skale (VAS) and pain was determined with the pain domain of the Nottingham Health Profile.

Results: The VAS value and pain scores didn't related to age, height, body weight, body mass index, DM and allodynia durations ( $p>0,05)$. The VAS value and pain score were also not correlate with each other $(\mathrm{p}>0,05)$. There were negative and poor associatons between SMWT distance with pain score and VAS value $(\mathrm{p}>0,05)$. The PM score was correlated to pain score $(\mathrm{p}<0$, $05)$, but not to VAS value ( $p>0,05)$. There were relationships between diabetes duration with age $(\mathrm{p}<0,01)$ and SMWT distance $(\mathrm{p}<0,05)$.

Conclusion(s): This data suggests that physical activity doesn't correlate with allodynia specific to peripheral neuropathy, but it associates with the pain in the other regions of body. These relationships should be evaluated in a larger sample group.

\section{1 \\ Rehabilitation of Patients with Stroke}

Sameniene J., Varzaityte L.

Lithuanian University of Health Sciences, Lithuania

Background: Aim of study to evaluate cognitive and psychomotor disorders and the disturbances of activities and participation of patients with stroke and to asses the effectiveness of rehabilitation

Methods: The contingent of the examined patients consisted of 30 patients with stroke rehabilitated in the

Department of Neurorehabilitation at Kaunas University of Medicine Hospital. The average age of rehabilitees was $63.33 \pm 13.2$ yrs. During the study we evaluated their cognitive functions with MMSE, handgrip strength with dynamometer, reaction time and frequency of movements with reactiometer, activities and participation disorder was valued by International classification of functioning, disability and health.

Results: After the applied course of individualized rehabilitation programme, cognitive functions of the patients significantly improved. The increased in cognitive functions estimated with MMSE was $5.4( \pm 1.6)$ scores $(\mathrm{p}<0.001)$. The examination shoved that before rehabilitation it was significant difference between right and left hemisphere damage and patients movement frequency and handgrip strength. After rehabilitation it was estimated significant change in patients reaction time, handgrip strength and right hand movement frequency $(\mathrm{p}<0.001)$. We determined, that changing and maintaining body position, applying knowledge activities was most confused, general interpersonal interactions was confused moderately, communication producing was confused least.

Conclusion(s): The results of the research shoved, that there was correlation in patients with stroke mental state and reaction time and changing and maintaining body position, applying knowledge activities after applied rehabilitation.

\section{1 \\ Factors Contributing to Balance Deficits in People with Idiopathic Parkinson Disease: A Systematic Review}

Diab A.K., Hale L., Waters D.

Otago University, School of Physiotherapy, New Zealand

Background: Postural instability is a cardinal sign of Parkinson's disease but the reason for postural instability is not fully understood. The aim of this systematic review was to critically explore factors that may contribute to postural instability in idiopathic Parkinson's disease.

Methods: Ten databases were searched from their establishment until March 2012 for studies investigated outcomes of balance, and addressing factors postulated to cause postural instability in patients with idiopathic Parkinson's disease. Only studies which had an experimental or observational design were included. The Generic Appraisal Tool for Epidemiology (GATE) checklist was used to appraise the methodological quality of selected articles. Data from those studies rated as of strong evidence were evaluated to identify the factors associated with reduced balance in idiopathic Parkinson's disease.

Results: The search identified 59 studies, of which six were rated to have strong evidence data. Most studies $(n=43,72.8 \%)$ used posturography to measure balance. The following causative factors were investigated: rigidity, and stiffness of postural muscles causing response deficits to perturbations ( $\mathrm{n}=14,23.7 \%$ ), sensory disorders, bradykinesia, postural adjustments disorders, systemic dysfunction, and L-Dopa complications. Studies with strong evidence $(n=6)$ reported L-Dopa induced dyskinesia, bradykinesia, postural hypotension and cognitive impairment contributing to reduced balance.

Conclusion: There is limited evidence for identified causative/ associated factors for postural instability in Parkinson's disease and it may be that reduced balance is a result of a complex interplay of a number of factors. Further research is suggested to elucidate the factors involved in postural instability to appropriately target interventions to improve balance. 
228

The Application of Event-Related-Potential P300 in

Vascular Cognitive Impairment-No Dementia

Patients Before and After Cognitive Rehabilitation Training

Feng $H$., Zhu Q.

Affiliated Hospital of Qingdao Medical College, China

Background: Vascular cognitive impairment has high incidence and is currently treatable. Event-related potentials P300 have a large application in the evaluation of cognitive processes. Few studies exist on the event-related-potential P300 and patients with vascular cognitive impairment-no dementia VCIND. We aim to study the effect of cognitive scale and auditory event-related potential P300 on VCIND patients before and after cognitive rehabilitation training and to further explore the application value of P300 in the early diagnosis of vascular cognitive impairment.

Methods: 57 patients meeting the diagnostic criterion of VCIND were randomly allocated to treatment group $(\mathrm{n}=30)$ and control group $(n=27)$. Another 30 healthy volunteers without cognitive deficits recruited in the same period were used as normal control. Each participant was evaluated by auditory-P300, the Cognitive Assessment Scale scores including the Mini-Mental State Exam (MMSE) and the Montreal Cognitive Assessment (MoCA) scale respectively before and after the treatment. These measurement indicators were analyzed and evaluated taking into account of factors such as education, age and so on.

Results: Before the treatment, compared with the normal control, the P300 latency was significantly longer in the VCIND group $\mathrm{i}^{1} 1{ }^{\wedge} \mathrm{P}<0.01 \mathrm{i} 1 / 4 \%$ and the amplitude was significantly lower in the VCIND group $\left(\mathrm{P}<0.05 \mathrm{i}^{1} \frac{1}{4} \%\right.$; The Cognitive Assessment Scale scores of MMSEã $€$ MoCA and ADL(Barther Index) were significantly lower in the VCIND group; However, there were no significant difference with respect to naming ã€ speech and orientation (P $>0.05 \mathrm{i}^{1} / 4 \%$. The P300 latency was negatively correlated with age $\mathrm{i}^{1} 1{ }^{\wedge} \mathrm{P}<0.05 \mathrm{i}^{1} / 4 \%$ and positively correlated with years of education $\mathrm{i}^{1 / 1}{ }^{\wedge} \mathrm{P}<0.05 \mathrm{i}^{1 / 4} \%$. After cognitive rehabilitation therapy, compared with before the treatment, the P300 latency in VCIND treatment group was significantly shorter while the amplitude was significantly higher $\mathrm{i}^{1 / 4} 4^{\wedge} \mathrm{P}<0.05 \mathrm{i}^{1 / 1} 4 \%$ and the cognitive scale scores increased significantly $\mathrm{i}^{1 / 4}{ }^{\wedge} \mathrm{P}<0.05 \mathrm{i}^{1} / 4 \%$. After cognitive rehabilitation therapy, compared with the VCIND control group, the change of each indicator was more significant in the treatment group $\ddot{1}^{1} / 4{ }^{\wedge} \mathrm{P}<0.05 \mathrm{i}^{1} / 4 \%$ o than the control group.

Conclusion(s): Event-related-potential P300 can objectively reflect early cognitive dysfunction in the vascular cognitive impairment-no dementia patients. Early cognitive training can improve the cognitive function of patients with vascular cognitive impairment.

\section{Session P7: Neuroimaging Studies}

13

\section{Enhanced Prefrontal Activity and Concentration After Play Around: A fNIRS Study}

Yanagisawa $\mathrm{H}$.

Physical Fitness Research Institute, Japan

Background: Several studies have shown that even moderate exercise would improve cognitive function. However, the region of the brain affected by exercise that gets activated in a cognitive task has not yet been clarified in children. Multichannel nearinfrared spectroscopy (NIRS) offers a brain imaging tool to study hemodynamic changes during activation of specific brain loci. And unlike other imaging techniques, such as fMRI and PET, fNIRS is appropriate for functional cognitive studies relevant to sports science since no movement restraint is required.

Methods: In this study, 18 healthy subjects (age: 5-6) were asked to perform the task. This task using bead make value concentration. During the task we measured changes in concentration of oxy- and deoxy-hemoglobin. The concentration of change were measured during the task by fNIRS. In this study we set the exercise session to 20 minutes. Before and after exercise they perform the task.

Results: After the exercise there was significant change in task performance. The cerebral blood flow in response to the task task also was increased in around the dorso-lateral prefrontal cortex.

Conclusion(s): In this study, we applied NIRS measurements to assess the neural correlates underlying the cognitive effect of an acute bout of exercise target children. These results strongly suggest that exercise improves cognitive function which would probably due to the more efficient neuroactivation at the DLPFC and their vicinity.

40

\section{Atheromatous Plaques in the Aortic Arch or Supra- Aortic Arteries Are Not a Risk Factor of Cryptogenic Stroke}

Cui X., Jiahe X., Simiao W., Liu M.

West China Hospital, Sichuan University, China

Background: Atheromatous plaques dropping off from the arterial wall of aortic arch or supra-aortic arteries (innominate artery, bilateral subclavian arteries and bilateral common carotid arteries) may cause embolic stroke and may account for part of the cryptogenic stroke. But the incidence of these plaques in the stroke patients especially the cryptogenic stroke patients is unknown. The objective of this study was to investigate whether atheromatous plaques in the aortic arch or supra-aortic arteries are a risk factor of cryptogenic stroke. 
Methods: A consecutive series of patients with acute ischemic stroke were divided into case group (cryptogenic stroke) and control group (stroke of determined etiology). The plaques were detected by CTA and assessed by an experienced radiologist and a neurologist. Clinical history and risk factors of vascular event were also collected.

Data analysis was carried with SPSS 19.0. Proportions between groups were compared by the Chi-Square test. The importance of atheromatous plaques for cryptogenic stroke was assessed by logistic Regression analysis controlling of age, sex and other risk factors of vascular event.Results were expressed as ORs with 95\% CI.

Results: In our pilot study, 92 patients $(60.98 \pm 15.45$ years $)$ were investigated. Atheromatous plaques presented in $68.57 \%$ $(24 / 35)$ of case subjects and $56.14 \%(32 / 57)$ of the controls. The complex plaques(mobile, ulcerated, or $\geq 4 \mathrm{~mm}$ thick) rate in the two groups was $47.37 \%$ VS $66.04 \%$. The incidence of plaques in the aortic arch and supra-aortic arteries is not significantly associated with cryptogenic stroke (OR 1.705, 95\%CI 0.704 to $4.13, \mathrm{p}=$ 0.236 ), even after adjusting the confounding factors.

Conclusion(s): Atheromatous plaques in the aortic arch or supra-aortic arteries are not a risk factor of cryptogenic stroke. As the sample size of this pilot study is small, large-scale studies are needed to further explore the role of aortic arch or supra-aortic plagues in cryptogenic stroke.

\section{7}

\section{Treatment of the EEG: A Hung Jury

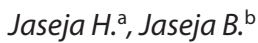 \\ ${ }^{\mathrm{a} G} \mathrm{R}$ Medical College, India; ${ }^{\mathrm{b}}$ Madhav Institute of Technology and Science, India}

EEG is a non-invasive neuroimaging technique that is widely accepted as mainstay in the investigation and management of epilepsy especially for identifying an epileptogenic region. Significant concordance between focal EEG interictal slow-wave discharges and focal blood oxygen level-dependent (BOLD) activation on fMRI has been detected and which has been found to correspond to the epileptogenic area. The interictal epileptiform discharges (IEDs) detected by EEG are recognized as hallmarks of epilepsy; however, since they are also found to correlate well with local metabolic changes in the brain, the IEDs may be associated with localization-related alteration in functioning of the affected cortical neuronal pools. This is evidenced by several studies in which transient cognitive impairment has been observed during IEDs and suppression of which has resulted in cognitive improvement. Further, in recent microstructural and functional MRI studies of cognitive impairment, volumetric MRI and voxel-based morphometry have revealed significant volume losses in the seizure zones where as diffusion tensor imaging studies has shown loss of integrity of connections from the seizure focus to distant areas, thus highlighting the anatomical and functional disruptions in the region of seizure focus, the region of generation of the IEDs. These observations have led to a dilemma on treatment of the IEDs or rather treatment of the EEG with emergence of one school of thought advocating the treatment of the EEG and the other school challenging it. The advocates of treatment of the EEG hold their ground on the non-benign nature and involvement of a substantial cortical area (at least $10 \mathrm{~cm} 2$ ) for scalp detection of IEDs along with the pathophysiology of generation of IEDs and their deleterious effects on cortical functioning while those against their treatment base their arguments strongly on overinterpretation and/or misinterpretation of the EEG. Uncontrolled reports and a few preliminary randomised controlled trials of suppression of IEDs by anti-epileptic drugs (AEDs) have yielded significant improvement in psychosocial functioning. Another recent study by Stecker (2009) has shown that an abnormal EEG is associated with increased morbidity and mortality even in diseases not directly warranting an EEG investigation. Thus, well designed prospective and retrospective studies are recommended in attempts to resolve the dilemma on treatment of the EEG.

\section{Session P8: Neurogenetics}

\section{3 \\ First Case of Spinocerebellar Ataxia Type 1 in a Mexican Female}

\author{
Garcia-Cruz D., Moran-Moguel C., Sanchez-Corona J., \\ Castañeda-Cisneros G. ${ }^{a}$, Sanchez-Garcia D.M. ${ }^{\text {b }}$, Orozco- \\ Gutierrez M.H. ', Nuñez-Reveles N., Alcaraz-Ochoa R. ', Davalos- \\ Rodriguez I. , Davalos-Rodriguez N. ', Ramirez-Garcia S. , \\ de Jorge L. ${ }^{\text {, }, ~ S a n ~ N i c o l a s-F e r n a n d e z ~} H .{ }^{e}$, Volpini $V^{f}$
}

anstituto Mexicano del Seguro Social, Mexico; 'bITESO, Mexico;

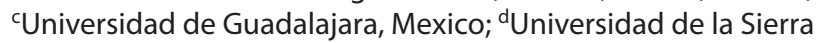
Sur, Mexico; ${ }^{\text {IIDIBELL, Spain; }{ }^{\mathrm{I} I D I B}, \text { Mexico }}$

Background: Spinocerebellar ataxia type 1 is an autosomal dominant disease caused by expansion of the CAG trinucleotide repeats in the ataxin-1 gene. Clinically characterized by cerebellar signs, upper motor neuron signs and extensor plantar responses, scanning speech, incoordination, slow motor-nerve conduction and choreiform movements in some instances. The aim of this study is to describe the first case affected of SCA1 in Mexico.

Methods: A female aged 24 year-old was studied due to gait ataxia, dysmetria, dysarthria, disdiadochokinesis, scanning speech and hypermetric bilateral saccades.

Results: Molecular analysis revealed a number of expansion repeats of $32 /^{*}$ exp.

Conclusion: In previous studies done in Mexican population there was not detected any patient with SCA1, so its frequency is unknown. 


\section{5}

\section{Clinical Study of Polish Patients with SPAST Gene Mutations}

Stepniak Iwona

Institute of Psychiatry and Neurology, Poland

Background: Hereditary spastic paraplegias (HSP) are a group of clinically and genetically heterogeneous, neurodegenerative disorders characterized by progressive spasticity of the lower limbs. The most common form of HSP is caused by mutations in the SPAST gene. The phenotype associated with mutations in the SPAST gene tends to be pure, however additional features may occur.

Methods: In a group of 59 patients with SPAST gene mutations, the diagnostic criteria for HSP were used according to Fink. All individuals were assessed by Spastic Paraplegia Rating Scale (SPRS) and classification used by Dürr et al. In a group of 27 from 59 patients, the cerebral, cervical and thoracic spines MRI, ophthalmological examinations as well as neurophysiological assessment were performed.

Results: We identified 11 microrearrangements (10 deletions and 1 duplication) and 17 point mutations in the SPAST gene. Age at onset ranged from 1 to 58 years (mean 18). SPRS scores ranged from 1 to 46 (mean 18). The progression of disability tended to increase with disease duration. $28 \%$ patients could not move without walking aids (disability score 4 or 5). 2 mutations carriers didn't complain of gait problems, but neurological examination detected evident signs of spasticity. Brain MRI showed white matter abnormalities (35\%), thinning of corpus callosum (14\%), cortical (25\%) and cerebellar (21\%) atrophy.

Conclusions: The present study revealed considerable variability in functional profile among the affected individuals. No significant differences in the clinical picture among patients with SPAST gene point mutations and microrearrangements were found. Furthermore almost all patients presented pure form of HSP, only in 1 patient peripheral neuropathy was identified.

\section{2}

Decreased Platelet Serotonin Level is Associated with Fatigue and Depression in Sjogren's Syndrome

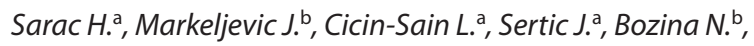
Barsic I. ${ }^{c}$, Erdeljic V. ${ }^{c}$, Henigsberg N. ${ }^{b}$,

aUniversity Hospital Zagreb, Croatian Institute for Brain Research, Croatia; ${ }^{\circ}$ Medical School University of Zagreb, Croatia; 'University Hospital Zagreb, Croatia

Background: Platelet serotonin level (PSL) is decreased in various inflammatory rheumatic disorders and is associated with the disease activity. We presume that PSL is decresed in primary Sjogren's syndrome (pSS) and is associated with fatigue and inflammation induced depression.

Methods: PSL was determined in 61 patients with pSS and 100 healthy controls by using spectrofluorimetric method. Clinical assesment was done in patients with pSS by using ESSDAI, ESSPRI, VAS, Beck depression inventory.

Results: PSL was significantly decreased in patients with pSS in comparisons to helathy controls. PSL is significantly associated with symptoms of chronic stress including fatigue and inflammation induced depression. PSl was not association with any standard or specific soluble markers of immunoinflammation including SE, CRP, ANA, ENA; SS-A and SS-B.

Conclusion: Fatigue and depression are associated with abnormalities in 5-HT system in pSS. Biopsychosocial model exist in pSS. Changes in 5-HT signaling occur in pSS.

\section{2 \\ Phenotypic Variability: A Case Study of 12 Year Old Identical Twins with Neurofibromatosis I}

Hartnett $E$.

NYU Langone Medical Center, USA

Background: The purpose of this case study is to explore the use of genetic and genomic information to guide the care of identical twins with Neurofibromatosis 1 .

Neurofibromatosis 1 (NF1) is one of the most common Mendelian disorders with an incidence of 1 in 3, 500 people. NF1 is a neurocutaneous disorder caused by a mutation on chromosome 17. It is inherited as an autosomal dominant with a $50 \%$ rate of new mutations, age dependent penetrance and variable expression. The NF1 patient has many physical and psychosocial concerns which need medical attention. The age dependent penetrance and variable expression are factors which influence the importance of continued surveillance. The study of identical twins is a valuable way to explore the factors related to the phenotypic expression of the disease.

Methods: These twins were seen in an urban outpatient pediatric oncology clinic. This was reported as the first case of monochorionic diamniotic twins mosaic for NF1 with one twin unaffected at age 12 . The family genogram shows no other family members affected by NF1. This data was collected by interview with the family and geneticist, genetic testing and chart review.

Results: Both twins have the genotype for NF1, the same mutation deletion on chromosome 17. Mosaicism was found in $25 \%$ of the blood cells in both twins and in $15 \%$ of the saliva of Twin A and $8 \%$ of the saliva of Twin B. Twin A has the phenotype, Twin B does not.

Conclusion(s): The healthcare professional has a unique role in educating the patient and family. Both twins need to be monitored closely, Twin A for progression of symptoms, Twin B for development of symptoms. Future research needs to be developed to determine what genetic, personal or environmental factors promote or prevent the expression of the phenotype in NF1. 


\section{2 \\ Mitochondrial Genome Profile in Demyelinating Diseases}

Durastanti V.a , Monaco A. ${ }^{\mathrm{b}}$, Marincola F. ${ }^{\mathrm{b}}$, Wang E. ${ }^{\mathrm{b}}$, Caronti B. ${ }^{\mathrm{a}}$, Cortese A. ${ }^{a}$, Berardelli A. ${ }^{a}$, Millefiorini $E^{a}{ }^{a}$

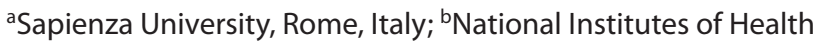
Bethesda, USA

Background: Multiple sclerosis (MS) and neuromyelitis optica (NMO) are chronic inflammatory diseases of the central nervous system (CNS). These pathologies share clinical similarities with Leber hereditary optic neuropathy (LHON), which is primarily due to mutations of mitochondrial (mt) DNA. Mitochondrial genetic variations may influence susceptibility to develop MS and NMO. In our study we aimed at determining a possible correlation between mtDNA specific patterns and demyelinating diseases involving medulla and optic nerve.

Methods: Three cohorts, [13 patients with relapsing-remitting (RR) MS, 4 patients with NMO, 1 patient with myelitis (My), 2 patient with optic neuritis (ON), and 7 healthy controls (HC)] were sequenced for the full $16 \mathrm{Kbs}$ of the mtDNA genome length. Our strategy allowed us to cut off variants occurring also in HC showing no clinical impact on diseases development.

Results: In our cohort of patients were detected six nonsense mutations, causing early stop-codon formation, and nine previously described variants, associated with demyelinating/degenerative disease of CNS.

Conclusion: Some variants identified in the study are linked to disease development through known and previously described mechanisms. For other truncating mutations detected leading to incomplete proteins involved in OXPHOS complexes, we could only speculate their role in demyelinating diseases development, since they have been reported for the first time in this study and further functional investigation are required.

\section{Session P9: Neuropharmacology}

21

\section{Evaluation of Borassus Flabellifer Seed Embryo for the Elevated Plus-Maze, Open-Field, Forced Swim and Tail Suspension Tests for the Assessment of Anxiety-Related Behavior in Albino Mice}

Karuppusamy A., Parimelazhgan T.

Bharathiar University, India

Background: The palmyrah (Borassus flabellifer L.) belonging to Arecaceae, grows wild from the Persian Gulf to the CambodianVietnamese border. It is commonly cultivated in India, Southeast Asia, Malaysia and occasionally in other warm regions including
Hawaii and southern Florida. It has innumerable medicinal uses for all parts of the Palmyra palm. The plant is used relieve biliousness, dysentery and gonorrhea, roots are diuretic and anthelmintic and a decoction is given for certain respiratory diseases. The seed shoot and seed embryos are used as antioxidant, anti-inflammatory, rheumatism.

Methods: The aim of this study was to study the ameliorative effects Borassus flabellifer seed embryo on stress-induced anxiety and depression. The study was carried out using male albino rats $(22-30 \mathrm{~g})$. The effect of B. flabellifer seed embryo was evaluated for anxiety and depression using elevated plus maze (EPM) test, open field test (OFT), forced swim test (FST), and tail suspension test (TST). Restraint stress ( $3 \mathrm{~h} /$ day for six consecutive days) induced a significant reduction in both the percentage number of entries and time spent in open arms in EPM, and these changes were reversed with post-treatment of aqueous extract of B. flabellifer seed embryo (100, 200, 300 and $400 \mathrm{mg} / \mathrm{kg}$ for 6 days).

Results: Restraint stress-induced (a) increased latency and (b) decreased ambulation and rearing were also reversed by B. flabellifer seed embryo in OFT. A significant increase in immobility period was observed in FST and TST after restraint stress. B. flabellifer seed embryo significantly reduced the immobility times of rats in FST and TST. B. flabellifer seed embryo possesses anxiolytic and antidepressant activities.

Conclusion(s): In the entire behavioral test, B.flabellifer seed embryo shows higher protection. It may be concluded, therefore, that seed embryo is more protective in anxiety and depression.

32

\section{Loss of Histaminergic Modulation of Thermoregulation and Energy Homeostasis in Obese Mice}

Tabarean J.

The Scripps Research Institute, USA

Background: Histamine acts centrally to increase energy expenditure and reduce body weight by mechanisms not fully understood. It has been suggested that in the obese state hypothalamic histamine signaling is altered. Previous studies have also shown that histamine acting in the preoptic area controls thermoregulation. We aimed to study the influence of preoptic histamine on body temperature and energy homeostasis in control and obese mice.

Methods: We have used telemetry and metabolic chambers to characterize the influence of histamine receptor $(\mathrm{H} 1, \mathrm{H} 2$ and $\mathrm{H} 3$ ) agonism in the median and medial preoptic nuclei in control and diet-induced obese mice. The agonists were microinjected locally. We have also characterized the expression of uncoupling proteins in thermogenic tissues (brown and white adipose tissue as well as skeletal muscle).

Results: Activating histamine receptors in the preoptic area by increasing the concentration of endogenous histamine or by local injection of specific agonists induced an elevation of core body temperature and decreased respiratory exchange ratio (RER). In addition, the food intake was significantly decreased. The hyper- 
thermic effect was associated with a rapid increase in mRNA expression of uncoupling proteins in thermogenic tissues, the most pronounced being that of uncoupling protein (UCP) 1 in brown adipose tissue and of UCP2 in white adipose tissue. In diet induced obese mice histamine had much diminished hyperthermic effects as well as reduced effect on RER. Similarly, the ability of preoptic histamine signaling to increase the expression of uncoupling proteins was abolished. We also found that the expression of mRNA encoding the $\mathrm{H} 1$ receptor subtype in the preoptic area was significantly lower in obese animals.

Conclusion(s): These results indicate that histamine signaling in the preoptic area modulates energy homeostasis by regulating body temperature, metabolic parameters and food intake and that the obese state is associated with a decrease in neurotransmitter's influence.

\section{0}

\section{Co-Morbidity of Traumatic Brain Impairment in Alcoholism: Efficacy of Application of Finlepsin}

\section{Bokhan Nikolay}

Mental Health Research Institute, Russia Federation

Background: Co-morbid traumatic brain impairment in alcoholism (F10.2+CCI) reduces application of finlepsin for stopping of affective (dysphoric) manifestations of withdrawal syndrome (WS) because of cerebral complications This shortage is absent during application of retard form of finlepsin 200 (FR200).

Material and methods: We have studied efficacy of FR200 in

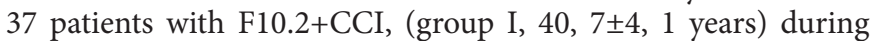
therapy of dysphoric manifestations of WS (week 1) and during post-withdrawal period (weeks 2 and 3 ). In the first group preparation was administered $600 \mathrm{mg}$ a day during 3 weeks of onset of WS. In group II ( $\mathrm{n}=15$, age $38,6 \pm 3,8$ лет) FR200 was not administered. We used scales HAM-D (17) и CGI-I in 4 points: screening (1), assessment at baseline (2), days of therapy 7 and 21 (visits 3 and 4).

Results of study of clinical efficacy of finlepsin-retard in sufferers in group I with impairment in question have revealed, besides known anticonvulsive, anti-dysphoric, and analgesic; vegetostabilizing effects of its therapeutic action have been detected. Among affective disturbances the most withdrawal syndrome-related action is found during dysphoric manifestations (72, $2 \%$ of efficacy). Number of patients with $50 \%$ reduction of scores according to scales HAM-D (17) during visit $3-68,7$ (in group $2-34,6$; $\mathrm{p}<$ $0,05)$ and $37,5 \%(30,8 \%)$ during visit $4-93,7(76,9 ; \mathrm{p}<0,05)$ and $78,1 \%(65,4)$, respectively. From clinical manifestations of toxicogenic effects of ethanol in the structure of alcohol withdrawal syndrome cerebral (cephalgia, diencephalic paroxysms), myofascial (local muscular-tonic hyperkinesias like crampi) symptom complexes possess the most severe sensitivity.

Conclusion: FR200 is effective in the case of dysphoric manifestations during withdrawal syndrome.

\section{2 \\ Neuroprotective Effects of Chloroform and Petroleum Ether Extracts of Nigella Sativa in Stroke Model of Rats}

Akhtar M., Najmi A.K., Hanam R., Ahmad A., Mujeeb M., Aqil M., Ismail M.V.

Jamia Hamdard, Hamdard University, India

Background: Many herbal drugs in different indigenous system of medicine claim to have beneficial effects but not extensively evaluated for stroke (cerebral ischemia). Nigella sative has been reported to demonstrate many pharmacological activities. The present study was carried out to establish its neuroprotective role using chloroform and petroleum ether extracts $(400 \mathrm{mg} / \mathrm{kg}$, per orally daily), administered for one week in cerebral ischemia using middle cerebral artery occlusion model in rats.

Methods: Cerebral ischemia was induced by middle cerebral artery occlusion (MCAO) for 2 hours followed by reperfusion for 22 hours in rats. After 24 hours of ischemia, grip strength, locomotor activity tests were performed. After behavioral tests, animals were immediately sacrificed. Infarct size was measured followed by markers of oxidative stress parameters.

Results: There was improvement in locomotor activity and grip strength in both chloroform and petroleum ether extracts pre-treated rats $(\mathrm{p}<0.01)$. Infarct size was reduced in both extracts pretreated rats as compared with MCAO rats significantly $(\mathrm{p}<$ 0.01). Thiobarbituric acid reactive substance (TBARS) were elevated, glutathione (GSH) and antioxidant enzymes viz. superoxide dismutase (SOD) and catalase levels were reduced following MCAO. Pre-treatment of both extracts of Nigella sativa showed the reduction in TBARS, elevation in glutathione, SOD and catalase levels as compared with MCAoccluded rats.

Conclusion(s): The neuroprotective effects observed in the present study by both the extracts could be due antioxidant, free radical scavenging and anti-inflammatory properties of Nigella sativa.

234

Roles of PI3K/Akt and Nrf2-Keap1 Pathways in the Hormetic Effect of Z-Ligustilide on PC1 2 Cells Against Oxygen and Glucose Deprivation

Rong J. ${ }^{\mathrm{a}}$, Qi H. ${ }^{\mathrm{a}}$, Han Y. ${ }^{\mathrm{b}}$

aUniversity of Hong Kong; ${ }^{\text {}}$ The Hong Kong Polytechnic University, China

Background: Phytochemicals are widely used to ameliorate neurological disorders. The aim of this study was to characterize the hormetic effect of Z-ligustilide on PC12 cells against oxygen glucose deprivation (OGD).

Methods: Through the analysis of the cellular proteins by Western blotting, we focused on the interactions of Z-ligustilide with the pro-survival signals mediated by phosphatidylinositol 3-kinase (PI3K) and transcription factor nuclear factor-erythroid 
2 p45-related factor 2 (Nrf2) pathways. We also examined the effect of Z-ligustilide on the intracellular redox signaling system involving reactive oxygen species (ROS) and glutathione (GSH).

Results: We found that Z-ligustilide not only triggered stress response by causing ROS formation and transient GSH depletion, but also activated survival-promoting signals via cross-talking of $\mathrm{PI} 3 \mathrm{~K}$ and Nrf2 pathways. Importantly, we demonstrated that Z-ligustilide preconditioning protected PC12 cells from OGDinduced injury either at a low concentration for a prolonged period of time or at a high concentration for a short period of time. It is possible that mild preconditioning stimulates moderate ROS production, but effectively activates hormetic signals and induces stress responsive genes. In contrast, higher concentrations of Z-ligustilide could be toxic over a prolonged period of time due to massive ROS production.

Conclusion: The effect of Z-ligustilide may be regulated by a biphasic hormetic mechanism involving initial induction of oxidative stress and subsequent activation of stress response gene expression.

\section{0 \\ Synthesis and Influence of Two Quinoxalinone Derivatives on Anxiety- and Depressive-Like Responses in Wistar Rat}

Nakache R. ${ }^{a}$, El Hessni A. ${ }^{a}$, Benazzouz B. ${ }^{a}$, Lakhrissi B. ${ }^{\mathrm{b}}$, Ouichou A. ${ }^{a}$, El Mrabet F.Z. ${ }^{a}$, Mesfioui A. ${ }^{a}$

aLaboratory of Genetics, Neuroendocrinology and Biotechnology, Unit of Nervous and Endocrine Physiology, Department of Biology, Faculty of Sciences, University Ibn Tofail, Kenitra, Morocco; 'baboratoire de Chimie

d'Agroressources et Génie des Procédées, Faculté des Sciences, Kenitra MAROC, Morocco

Background: Anxiety and depression seem to be associated with modification in central neurotransmission deterioration of monoaminergic systems (norepinephrine: NA, dopamine: DA and serotonin: 5HT). The use of antidepressants like clomipramine (CLMP), an inhibitor of 5HT uptake and anxiolytics like diazepam (DZ), a potentialisator of the GABAergic transmission, seems effective to cure these syndromes. Though, Benzodiazepines (Bzs) are well-established, clinically useful anxiolytics, but some effects that are characteristic of these drugs, particularly sedation and muscle relaxation, have been considered unwanted side effects. The goal of this study was to test the hypothesis that subchronic administration of quinoxalinic compounds would induce significant behavioural changes in Wistar rats.

Methods: For this purpose, two new quinoxalinic derivatives (2 $(1 \mathrm{H})$-quinoxalinone and 6 -nitro-2 $(1 \mathrm{H})$-quinoxalinone) have been synthesized adopting Hong method (Hong 2000) and investigated for some neuropharmacological effects (anxiety- and depressive-like responses) in rats. The present experiment sought to determine whether treatment with the compounds produces changes in affective responses. The rats were daily injected intraperitoneally; the anxiolytic and antidepressant standard group rats were administered with DZ $(1 \mathrm{mg} / \mathrm{kg})$ or CLMP $(2 \mathrm{mg} / \mathrm{kg})$ respec- tively. In the experiment using quinoxalinic derivatives, rats were administered with (6-nitro-2 $(1 \mathrm{H})$-quinoxalinone or $2(1 \mathrm{H})$-quinoxalinone) $(30 \mathrm{mg} / \mathrm{kg})$. Appropriate control studies were performed administering vehicle in place of drug. After two weeks in treatment conditions, the rats underwent a battery of behavioural tests (open field and elevated plus maze) to measure anxiety- and (forced swimming test) to measure depressive-like responses. To eliminate any lingering olfactory cues, the pieces of test equipment were thoroughly cleaned by using water and $20 \%$ alcohol followed by thorough drying before each rat was tested. Data are expressed as the means \pm standard error of the means (S.E.M.). To determine the differences between experimental groups statistical analysis was performed by analysis of variance (ANOVA) 1st order followed by a post-hoc tests (Fisher LSD) or Student test " $t$ ". Intergroup differences were considered significant when $\mathrm{p}<0.05$, very significant when $\mathrm{p}<0.01$ and highly significant at $\mathrm{p}<0.001$.

Results: The results indicate that the sub-chronic injection of 6-nitro-2 $(1 \mathrm{H})$-quinoxalinone showed obvious anxiolytic and antidepressant effects, respectively, measured in the behavioral tests of EPM and FST. The 6-nitro-2 $(1 \mathrm{H})$-quinoxalinone showed a comparative anxiolytic effect in rats as $\mathrm{Dz}$ and a comparative antidepressant effect as CLMP. The $2(1 \mathrm{H})$-quinoxalinone significantly reduced depressive-like responses as evaluated in FST, whereas any anxiolytic effect was shown as measured in OF and EPM. Locomotor activity levels in OFT and EPM were unaffected by treatment.

Conclusion(s): In conclusion, these data allowed us to make an observation on structure-activity relationships. The results of behavioural tests seem to confirm that for the anxiolytic-like activity, an electron-withdrawing substituent in the benzene moiety is necessary. In fact the compound maintaining the electronwithdrawing group (NO2) in the benzene moiety displayed both anxiolytic- and antidepressive-like effects.

\section{7 \\ Role of P2Y2 Receptors in Neuropathic Pain and Sex Difference}

Xu J., Susanne H., Niklas J., Gupta S.

Copenhagen University Hospital, Glostrup, Denmark

Background: Little is known about the contribution of purinergic system in the trigeminal ganglions (TG) to transduction of painful stimuli. $\mathrm{P} 2 \mathrm{Y} 2$ receptor is one of the $\mathrm{P} 2 \mathrm{Y}$ family of purineand pyrimidine-activated $G$ protein-coupled receptors in sensory ganglia. P2Y2 was the only P2Y receptor that showed an increase in DRG mRNA levels in an inflammation. P2Y2 (-/-) mice had significant deficits in thermal sensation, despite normal distribution of capsaicin receptor TRPV1. These results suggested that P2Y2 receptor in TG may be involved in certain types of neuropathic and inflammatory pain.

To study in-vitro role of $\mathrm{P} 2 \mathrm{Y} 2$ in pain signalling in trigeminal vascular system, P2Y2 (-/-) mice were used as a model. Calcitonin gene-related peptide (CGRP), a potent vasodilatory peptide, released after activation of afferents was used as a marker of pain. 
Specific stimuli Capsaicin and KCL were used to study the interactions of P2Y2 - TRPV1 and P2Y2 - K+ channels, respectively. Males and females under same experimental setup were used to study. Sex difference was studied.

Wild type (WT) or P2Y2(-/-) BALB/cJ mice (25g) were used. Trigeminal vascular components dura mater and TG were freshly isolated, CGRP release was analysed by ELISA after addition of capsaicin (10 nM-1 microM) or KCL (20 m-100 mM). All experiments were repeated $4-5$ times. One way ANOVA with Dunette's test was used to determine whether the effect by single compounds is significantly different from the controls. Graphpad Prism 5.0 for Windows (Graphpad Prism Software, San Diego, CA, USA) was used for statistical analysis.

Results: CGRP released from dura mater and TG was significantly reduced in P2Y2(-/-) males and females when compared to WT, regardless the stimulus was capsaicin or KCL.

When incubation with KCL, WT females released higher CGRP in dura mater than WT males, whereas it was lower in TG.

No sex difference was found in WT dura mater, except that a faint decrease in CGRP was shown in females only in exposure to 10-8 M capsaicin.

Conclusion(s): The study demonstrated that P2Y2 in trigeminal vascular system participated in CGRP regulation, which may contribute to neuropathic pain and its critical role in the transient receptor potential vanilloid-1 ion channel proteins (TRPV1) and $\mathrm{K}+$ channel normal functions. Sex hormone alone may not sufficient in P2Y2-mediated potentiation in dura mater. This may have implications for the pathophysiology and treatment of neuropathic and inflammatory pain.

(in this section, importance of results of the study for practice and/or future research is briefly described).

\section{4 \\ Effects of Histamine H3 Receptor Ligands on MK-801 Induced Locomotor Activity with Dopamine and Histamine Estimation in Rat Brain by LC-MS-MS Method}

\section{Akhtar M. ${ }^{\mathrm{a}}$, Mahmood D. ${ }^{\mathrm{a}}$, Khanam R. ${ }^{\mathrm{a}}$, Najmi A.K. ${ }^{\mathrm{a}}$, Pillai K.K. ${ }^{\mathrm{a}}$, Aqil M. ${ }^{\mathrm{a}}$, Mujeeb M. ${ }^{\mathrm{a}}$, Khuroo Arshad H. ${ }^{\mathrm{b}}$, Goswami D. ${ }^{\mathrm{b}}$ \\ a Jamia Hamdard (Hamdard University), India; ${ }^{\text {b Ranbaxy }}$ Gurgoan, India}

Background: Schizophrenia (SCZ) is a severely debilitating psychiatric disorder afflicting around $1 \%$ of the world's population characterized by symptoms such as hallucinations, delusions, and thought disorders. During recent past role of brain histaminergic neurons in SCZ has been considerably clarified and H3-receptors ligands remained centre stage for developing noble antipsychotic drugs (APDs). Histamine $\mathrm{H} 3$ receptor $(\mathrm{H} 3 \mathrm{R})$ is a third histamine receptor which acts as presynaptic auto receptor to control the release and synthesis of histamine (HA) in the central nervous system (CNS). Striatum has the highest densities of $\mathrm{H} 3 \mathrm{R}$. The determination of changes in basal levels of neurotransmitters in the brain has been important for testing the efficacy of putative drugs designed to treat disorders of the CNS.
Methods: The present study investigated the effects of repeated doses of two prototypical imidazole $\mathrm{H} 3$-receptor antagonists, ciproxifan (CPX) (3 mg/kg, i.p.) and clobenpropit (CBP) (15 $\mathrm{mg} / \mathrm{kg}$, i.p.) on MK-801 (0.2 mg/kg, i.p.) induced hyperactivity using activity monitoring system (TruScan 2.0 version, Coulbourn instrument USA) followed by determination of dopamine (DA) and HA concentrations of striatum and hypothalamus respectively in rats brain through LC-MS-MS methods.

Results: Our study showed MK-801 induced increases of DA concentrations in the striatum were reduced by both CPX and CBP administration while the increase of HA concentrations in hypothalamus were potentiated. Further, MK-801 induced hyperlocomotor behaviors were significantly decreased by both CPX as well as CBP.

Conclusion(s): The present study observed modulatory effects of both CPX as well as CBP on DA and HA levels in rat brain and reduction of MK-801 induced hyperlocomotor activities. Further research is required to confirm the potential role of histamine $\mathrm{H} 3 \mathrm{R}$ antagonists/inverse agonists in the symptomatic/add-on treatment of resistant forms of SCZ.

\section{Session P10: Neuropsychology}

\author{
176 \\ Cognitive Functions, Depression and Health Status in \\ Elderly Women

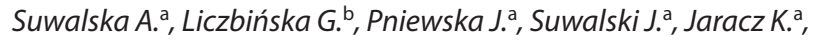 \\ Górna K. ${ }^{a}$, Pałys W. ${ }^{\text {, }}$ Lojko D. ${ }^{\text {a }}$

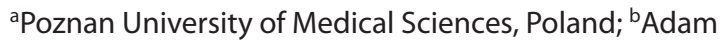 \\ Mickiewicz University, Poland
}

Background: The aim of the study was to explore the associations between depressive symptoms and health status and cognitive functions in the group of elderly women.

Methods: Sixty non-demented elder female (mean age $79.5 \pm$ 6.1 , range 60 - 85) living in Wielkopolska region entered the study. Dementia screening was performed using Mini Mental State Examination (MMSE). Depressive symptoms were assessed using Beck Depression Inventory (BDI) and cognitive functions by means of Stroop Test and Trail Making Test. All participants underwent a structured interview to collect information on demographics, education, physical health, and lifestyle.

Results: In the studied group significant correlations between TMT, Stroop Test part B results and age, as well as between education level and TMT scores were observed. Fifteen participants were depressed $($ BDI $>12 p)$. Physical health was categorized as 'healthy or two illnesses at most' (Hor2) v. 'multiple morbidity' (MM), the latter comprising those who had three or more somatic diseases. Depression intensity was higher in MM than in Hor2 group. In the Hor2 group depression intensity was associated with MMSE, TMT B and errors in Stroop test part B-errors, in the MM group only with errors in Stroop B test. 
Conclusion(s): The results of the study point to the impact of age and education on cognitive functions and suggest mutual interactions of depression and the physical health status as factors associated with cognitive impairment.

\section{Session P11: Neuropsychiatry}

\section{4 \\ Extrapyramidal Symptoms: After 5 Years on Second- Generation Neuroleptics}

Geml J., Cortese L., Velehorschi C., Villella G.

Windsor Regional Hospital, Canada

Background: Schizophrenia and related psychotic disorders have proven to be a debilitating disorder requiring complex treatment approaches. Historically, typical or first-generation neuroleptics such as haloperidol, chloropromazine, or fluphenazine were considered first-line treatment options for schizophrenia. In the 1970's, studies indicated 30\% of psychiatric outpatients were exhibiting signs of extrapyramidal symptoms (EPS). The development of second-generation "atypical" neuroleptics such as olanzapine, risperidone, or quetiapine were said to alter the dopaminergic effects resulting in a decrease in EPS. However, there exists a multitude of factors that may alter the development of EPS. Studies have shown that genetic predisposition may be a factor, with $5 \%$ of drug naïve patients exhibiting signs of a movement disorder. Additionally, use of a second-generation neuroleptic for a prolonged duration may elicit movement disorders similar to those seen in first-generation neuroleptics.

Methods: A five-year naturalistic outcome study in an underserviced community was conducted. All subjects had a primary diagnosis of a psychotic disorder and were treated with secondgeneration neuroleptics throughout their 5 years in the program. Subjects who consented to participate in the Wellness Program for Extended Psychosis completed regular assessment batteries. The test battery included the Simpson-Angus Scale (SAS), an established instrument to assess neuroleptic induced parkinsonism. The Extrapyramidal Symptom Rating Scale (ESRS) was developed to assess parkinsonism, akathisia, dystonia, and tardive dyskinesia and was also utilized to diagnose EPS. All assessments were completed by a board certified psychiatrist. Baseline and 5-year measures were utilized for data analyses.

Results: At the time of data collection 31 subjects had achieved 5 years of participation in the Wellness Program for Extended Psychosis. Of the 31 subjects, 26 had completed assessment sets for both baseline and 5 year measures, and were used in data analysis $(\mathrm{N}=26)$. There were 23 males and 3 females, ranging from 23 to 52 years of age. Overall results indicated an average score at baseline of 1.81 and 2.96 after 5 years for the SAS and 6.23 at baseline and 8.12 after 5 years for the ESRS. When dissected, 7 patients (27\%) were shown to display no signs of EPS throughout the course of treatment, while 3 patients (12\%) displayed consistent symptoms without any worsening over the 5 year period. Seven (27\%) individuals demonstrated an improvement in EPS rating scales while 8 individuals (31\%) were shown to experience a worsening of symptoms over the 5-year course of treatment with second-generation neuroleptics. One individual had conflicting results between the SAS and ESRS measures.

Conclusion(s): Schizophrenia is an illness that has been shown to require a complex medication regime. In this study, results contradict the belief that EPS is no longer a major concern, with the use of second-generation neuroleptics. Nearly one-third of patients on a second-generation neuroleptic, over a 5-year period, developed or experienced an exacerbation of EPS. Results include a description of the medications utilized and specific sub-types of EPS observed.

\section{0 \\ Epidemiology of Tardive Dyskinesias in Community Dwelling Schizophrenia Patients in Rural South India}

Udupi Shashank A., Channaveerachari Naveen K., Thirthahalli J., Udupi A., Kengeri Kishore K., Kudumallige Suresh K., Gangadhar Benagaluru N.

National Institute of Mental Health and Neurosciences, India

Background: Though the second generation antipsychotics are associated with lesser risk for Tardive Dyskinesia (TD), their prevalence is not known in community dwelling patients. Here we report on the prevalence and correlates of TD in a sample of community dwelling patients with schizophrenia.

Methods: The sample has come from Thirthahalli taluk (a local administrative subunit) South India. Our team has been running a community intervention program [titled 'Community Intervention in Psychotic Disorders' (CoInPsyD)] for the past seven years. Aim of this program is to identify, treat and follow-up all patients with schizophrenia in the taluk. Till date, 350 Patients have been identified. Data for this study comes from this cohort. Mini International Neuropsychiatric Interview is used for the purpose of diagnosing schizophrenia according to ICD-10 criteria; Positive and Negative Syndrome Scale (PANSS) is used to assess psychopathology; Tardive Dyskinesia Rating Scale (TDRS) is used to assess TD. 'Probable' TD was defined according to the SchoolerKane research criteria for TD. Severe TD was defined if the patient scored either five or six on any of the TDRS item.

Results: We assessed TD in 180 patients. Exclusions were for the following reasons: Migration ( $n=19)$, change of diagnosis ( $n$ $=10)$, death $(\mathrm{n}=28)$, lack of consent $(\mathrm{n}=8)$, not being able to contact patients due to wandering nature of their illnesses $(n=57)$ etc. Mean (SD) age of the sample was 43.5(11.5) years at baseline; females formed $49.4 \%(n=89)$ of the sample; $89(50 \%)$ were from lower socio-economic strata; 96(53.6\%) were married. Average number of years of education (SD) was 6.3(4.7) years; mean age at onset (of schizophrenia) was 28.4(9.9) years; Mean (SD) duration of illness was 180.9(117.6) months. Mean (SD) total score on PANSS was $69.6(25.1)$ at baseline; total duration of antipsychotic exposure was 53.4(26.4) months. Most of the patients were 
exposed to both typical as well as atypical antipsychotic medications. Prevalence of TD was 67/180 (37\%). However, the presence of severe TD was found only in five patients. When compared to patients without TD, those with TD were: significantly elder [age $46.3(12.8)$ vs $41.8(10.4)$ years respectively; $\mathrm{p}=0.01$ ] and had more severe current symptoms [mean current total PANSS score of 52.3 (20.0) vs 44.6(15.1) respectively; $\mathrm{p}<0.01$ ]. There was equal distribution of TD among males and females [33/67(49.3\%) vs $34 / 67(50.7 \%)$ respectively; $p=0.8]$. Duration of antipsychotic exposure was roughly the same in both the above mentioned groups [52.0 (26.7) months in those with TD vs 55.0 (26.2) months among those without TD; $\mathrm{p}=0.5]$.

Conclusion(s): Prevalence of TD in our community sample of schizophrenia patients was fairly high. However, the presence of severe TD was very low. In fact, most were unaware of their presence.

\section{Session P12: Basic and Translational Neuroscience}

\section{7}

\section{CXCL1 Deficit Associates with an Olig1 Increase in Subcortical Nuclei of the Taiep Rat}

Leon-Chavez B.A. a, Soto-Rodriguez G. ${ }^{\text {, }}$ Gonzalez-Barrios J.A. ${ }^{\mathrm{b}}$, Blanco-Alvarez V.M. ${ }^{\text {, }}$ Martinez-Fong D. ', Eguibar J.R. a , Ugarte A. , Piña-Leyva C. ${ }^{a}$, Torres-Soto M. ${ }^{a}$, Brambila E. ${ }^{a}$

aBenemerita Universidad Autonoma de Puebla, Mexico;

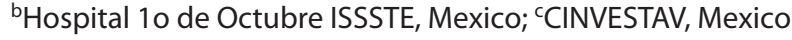

Background: The taiep rat develops hypomyelination at birth and progressive demyelination caused by both a cytoskeleton alteration of oligodendrocytes (OLs) and changes in expression and intracellular location of myelin proteins. GRO- $\alpha$ promotes proliferation and maturation of OL precursor cells (OLPs; expressing Olig1) by activating the CXCR2 receptor. IGF-1 promotes survival of OLs and neurons, and myelin synthesis. This work aims to evaluate whether GRO- $\alpha$ and IGF-1 are affected in the taiep rat.

Methods: We assessed the expression of GRO- $\alpha$, IGF-1, CXCR2 and Olig1 by RT-PCR and GalC and Olig1 by ELISA. Those variables were determined in the subcortical nuclei at different ages $(0.5,1,3,6$ and 8 months) of taiep and Sprague-Dawley rats.

Results: Our results showed that GRO- $\alpha$ mRNA levels significantly decreased $80.7 \pm 4.2 \%(\mathrm{p}<0.04)$ at 6 month-old of taiep rats when compared with those of controls. The levels of CXCR2 mRNA increased $135.4 \pm 18.9 \%$ in 1 month-old $(\mathrm{p}<0.0024)$ and $177.8 \pm 9.1 \%$ in 6 -month-old taiep rats when compared with controls. IGF-1 levels did not show significantly statistics difference in the subcortical nuclei at all ages. Olig mRNA levels were increased $53.6 \pm 3.1 \%$ in 15 days-old $(\mathrm{p}<0.01)$ and in $64.2 \pm 22.7 \% 6$ monthold $(\mathrm{p}<0.01)$ in the taiep rat when compared with SD rat. The decreased of GalC levels in subcortical nuclei of taiep rats possibly indicates a failure in the maturity response and survival of OLs.

Conclusion(s): The deregulation in GRO-a levels might affect the maturity of oligodendrocytes in the subcortical nuclei of the taiep rat, despite the increase in CXCR2 levels, which could further aggravate the demyelization process in the affected region of the taiep rat.

\section{1 \\ The Role of Chronic Stress in Early Secondary Progression of Multiple Sclerosis}

Beridze M., Devidze E., Shanidze L.

Tbilisi State Medical University Clinic, Georgia

Background: Chronic stress compromises the endogenous protective system of organism and may influence the course of multiple sclerosis (MS). Study purposed to investigate the role of chronic stress in secondary progression of MS.

Methods: Totally 52 secondary progressive MS (SPMS) patients, 24 from general population (1st group) and 28 (2nd group) from refuges suffering the chronic psychological and social stress have been investigated. Age at disease onset, disease duration, number of relapses, length of period until the secondary progression of disease and the Kurtzke Expanded Disability Status Scale (EDSS) scores were collected. Control comprised 15 healthy volunteers matched for age and sex. Brain was visualized by Magnetic Resonance Tomography (MRT- Siemens AVANTO-1.5Tesla). Depression was examined by Hamilton Depression Rating Scale (HAM-D17) and by Beck Depression Inventory (BDI-II). Blood free toxic radicals detected by Electron Paramagnetic Resonance Method (EPR). Statistics was performed by SPSS-11.0.

Results: In 2nd group SPMS developed in a significantly shorter period as compared to 1 st group $(6.3 \pm 2.1$ versus $14.2 \pm$ $3.8, \mathrm{p}<0.05)$. Depression was found in $22 \%$ of 1 st group and in $87 \%$ of 2 nd group (Ham-D $>17 / 18$ and BDI $>9 / 10$ ). Blood EPR specters of Lypoperoxiradical (LOO-) and superoxide anion (O2-) were increased in 2nd group compared to 1st group and control. Positive correlation was established between BDI index and LOOand $\mathrm{O} 2$ - data $(\mathrm{r}=+0.27$ and $\mathrm{r}=+0.18, \mathrm{p}<0.05)$.

Conclusion(s): Chronic stress may contribute to the promotion of free radical pathology in MS and thus, can stimulate the neurodegeneration. 
259

\section{A Novel Animal Model of Subarachnoid Hemorrhage- Induced Cardiac Dysfunction}

Munakata M., Kensuke K., Takahide A., Hiroaki S., Tabei R., Fukuda K.

KEIO University, Japan

Background: Cardiac dysfunction associated with subarachnoid hemorrhage (SAH) is a well-known phenomenon in neurosurgeon and cardiologist, which was first reported in the 1950s. Although over sixty years have passed from the early report, little is known about its mechanism. In addition, its animal model did not have been established.

This study is aimed to generate an animal model mimicking $\mathrm{SAH}$-induced cardiac manifestations to elucidate the mechanism of brain-heart pathway.

Methods: 8-weeks-old male Wistar rats were anesthetized and left carotid artery (LCA) was exteriorized through an incision in the neck. A nylon suture with sharpened tip was inserted into LCA. The tip of suture could accurately pierce the bifurcation of anterior and middle cerebral artery, resulting in SAH.

Electrocardiogram (ECG), echocardiography and left ventriculography were sequentially recorded pre and post SAH.

Results: $48 \%$ of SAH models $(\mathrm{N}=31)$ showed ST-segment elevation in ECG just after hemorrhage, and $45 \%$ of SAH models $(\mathrm{N}=35)$ showed depressed left ventricular contractility, peaked at 2 hours after hemorrhage.

In left ventriculography, compared with Wild type, SAH models were significantly decreasing left ventricular constriction.

The severity of SAH was evaluated by the hemorrhagic area of cerebral-base according to the established scale.

We revealed that the rate of cardiac abnormality is in proportion to the severity of hemorrhage. Mortality due to SAH was about $20 \%$, which is similar to human data.

Cardiac abnormalities induced by $\mathrm{SAH}$ were transient and normalized after 24 hours.

Conclusion(s): We successfully generated a new animal model presenting cardiac abnormalities related to SAH. This model might be a powerful tool to investigate the mechanisms of brainheart pathway.

\section{8}

\section{Platelet Aggregation in ALS Patients: A Unique Perspective}

Shrivastava M., Vivekanandhan S.

All India Institute of Medical Sciences, New Delhi, India

Background: Aggregability is one of the very important properties of platelets and assists in understanding their functional status. The functional status of platelets in turn helps to comprehend their internal mechanisms and activation of apoptotic cascade due to variety of factors such as increase in calcium, stress induced mitochondrial perturbances etc. Platelets are characterized as a systemic tool for detection of mitochondrial function pro- grammed cell death, glutamate excitotoxicity, adenosine5- triphosphate (ATP) contents and aggregability and activation in neurological disorders including ALS.Therefore, aggregation pattern of ALS and control platelets was assessed utilizing impedance based whole blood aggregometer reflecting their functional state.

Methods: Aggregation pattern of ALS and control platelets was assessed utilizing impedance based whole blood aggregometer using $\mathrm{ADP}$ as an agonist to specific platelet receptors. Recordings illustrates that increase in impedance is directly proportional to the mass of the platelet aggregates. Statistical analysis was done using unpaired student's t-test and a $\mathrm{p}$ value of $<0.05$ is considered significant.

Results: Our whole blood aggregometry results showed that impedance values were in the range of $11-17 \mathrm{ohms}$ (Mean $=15 \mathrm{ohms}$; $\mathrm{n}=17)$ in ALS platelets and $3-8 \mathrm{ohms}$ in control platelets (Mean $=$ 5.5 ohms; $\mathrm{n}=12$ ) when ADP was used as an agonist (conc. $5 \mu \mathrm{M})$. Our findings indicated that ALS platelets were more aggregated and exhibited their activated state as specified by their higher impedance values showing an inclination towards hyperaggregability. Both controls and ALS platelets showed no response to ATP standard (conc. 2 nanomoles) that was added to monitor ATP release from them. In vivo aggregation of ALS platelets was further vindicated by the results of confocal microscopy JC-1 labelling experiments and ALS platelets were more in number and mostly seen in clusters compared with single or less clustered control platelets.

Conclusion(s): Therefore, our whole blood aggregometry results may assist in understanding the fact that aggregation and greater impedence values seen in ALS platelets was probably due to increase in cytoplasmic free calcium level indicating mitochondrial dysfunction induced apoptosis eventually leading to shape change and platelet activation.

\section{Session P13: Nursing Care}

\author{
46 \\ Stress of the Neurology Nurses and Coping \\ Özbey H. \\ Turkey
}

Background: The aim of this study was to determine the stress level and its sources, and appraise the ways to cope with the stress. this section outlinesimportance, objectives/aims or hypothesis of the study.

Methods: This is a descriptive study. Data has been collected from the neurology clinics between May-September 2010. Simple size consisted of 100 neurology nurses. The demographic questionnaire, Nurse Stress Diagnostic Form, tress Appraisal and Coping technique, were used for collection data. The MannWhitney U test, Chi-square, percentage and Frequency distribution has been used to appraise the results by means of SPSS 12 data analysis software.in this section, design, subjects, and statistical analysis used in the study are briefly described. 
Results: The age of $35 \%$ of the neurology nurses participated in the research were 33 and over, 53\% were single, and 95\% were female. $52 \%$ were university graduate, $33 \%$ were the staff members of İstanbul University Cerrahpaşa Medicine Faculty, 46\% were Malatya İnönü University, Turgut Özal Medical Center. 55\% of the nurses have selected the nursing profession willingly while $74 \%$ deemed appropriate. $33 \%$ were employed 12 years and over, 52\% have selected the neurology service employed willingly, $72 \%$ were glad about their working environment, $48 \%$ were day and night member of staff, $54 \%$ were clinic nurse. $43 \%$ of the neurology nurses complain about the overly number of the patients to be cared, $37 \%$ of the nurses care the critical patients while $56 \%$ share the problems encountered with their nurse friends. Most frequently applied method used to cope with the stress during the stressed events was "self-reliant approach" (average:22, $65 \pm 2,73$ ) in this section, main results of the study are presented as clearly as possible.

Conclusion(s): It may be expressed that the "overly working load" for the neurology nurses who work at the clinics where the study performed is the cause of the highest stress factor and "selfreliant approach" was the most widely- used method of coping with the pressure among the other methods.(in this section, importance of results of the study for practice and/or future research is briefly described).

\section{7}

\section{Is Semiological Seizure Classification Practical Method for Pediatric Nurses?}

Isler A. ${ }^{a}$, Esenay Figen I. ${ }^{\mathrm{b}}$

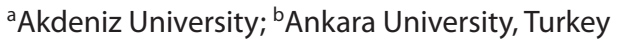

Background: In childhood epilepsy is a common neurological disease. Pediatric nurses often encounter childhood seizures while working in pediatric clinics and experience problems in defining seizures correctly.

Methods: The essential diagnosis in the management of an epileptic child is classification of the seizure. As seizures may develop instantly, early control of the seizure are among the principles of emergency treatment. It is of utmost importance that pediatric nurses define the seizure type and implement the necessary procedures immediately after occurrence of an epileptic seizure.

Results: A correct recognition of ictal seizure semiology is one of the first step in the diagnosis of epilepsy. Semiological Seizure Classification is an appropriate classification to recognition of seizure types easily by the pediatric nurses. By Luders and Noachtar designed Semiological Seizure Classification in 1998 is based exclusively on ictal seizure semiology, either as reported by the patient or observers or as analyzed directly during video monitoring. No electroencephalography findings or other test results influence the classification. In the classification, seizures are categorized in to five main categories. These are aura, autonomic seizures, dialeptic seizures, motor seizures and special seizures. In the literature there have been a series of studies on the practicability and reliability of this classification system in seizure diagnosis.
Conclusion(s): Semiological Seizure Classification will certainly offer a practical method for nurses in the pediatric clinics, and it has enabled to minimize the complications in connection with the definition of seizure types.

\section{Session P16: Healthcare}

\section{1 \\ Concept of Neuroresuscitation and Immediate Stroke Life Support}

Okudera H. ${ }^{a}$, Wakasugi M. ${ }^{a}$, Hashimoto M. ${ }^{\text {a }}$, Sakamoto M. ${ }^{\text {, }}$ Arishima T. ${ }^{\mathrm{a}}$, Sakamoto T. ${ }^{\mathrm{b}}$, Aruga . $^{\mathrm{c}}$

aUniversity of Toyama, Japan; b Teikyo University, Japan; 'Showa University, Japan

Background: We developed Neuroresuscitation (NR) as a new concept on neurological resuscitation and officialized as a chapter of Japan Resuscitation Council (JRC) Resuscitation Guidelines 2010. Furthermore, we refined Immediate Stroke Life Support (ISLS), half-day training course developed in 2006, based on NR guidelines 2010 and developed English version of ISLS.

Methods: NR is a important concept of JRC Resuscitation Guidelines 2010 for improving manegement of acute neurological disorders including stroke, epilepsy and post-resuscitation neurological deficits. ISLS was developed as training course for paramedic, ER physician, residents and nursing staff, by Japan ISLS committee consisting of Japanese Association of Acute Medicine and Japanese Congress of Neurological Emergencies in 2006. We refined ISLS based on NR.

Results: Japan ISLS committee completed refinement work of ISLS on 2011. Refined ISLS has been provided using workshops, faculty developments at related meetings and Internet. At the same time, we translate the refined ISLS in English for international curriculum sharing. English version was officialized as pre-congress course of 12th Internatinal Meeting on Simulation in Healthcare 2012, San Diego, CA, USA.

Conclusion(s): NR is a key concept on management of acute neurological disorders. ISLS is a dedicated training course of NR and is spreading rapidly for all prefectures in Japan. English version of ISLS is adapted as a part of education program of World Federation of Neurosurgical Societies and performed at Mongolia (2011), Nepal (2012) and Ethiopia (2012). Details of NR and ISLS will be presented. 
159

\section{Cognitive Impairment in Critical Illness Survivors - A Phenomenological Inquiry}

Tembo A.C. ${ }^{\text {, }}$ Higgins I. ${ }^{\mathrm{a}}$, Parker V. ${ }^{\mathrm{b}}$

aUniversity of Newcastle Australia, Australia; ${ }^{\text {bUniversity of New }}$ England, Armidale, NSW, Australia

Background: This paper disseminates the findings from a larger a study about the experiences of critically ill patients in intensive care (ICU). The aim was to describe and understand the experience of critical illness in to inform ICU practice. Technological advances and innovation in ICU practices driven by the Cartesian approach have increased survivors of critically illness. Patients' perspectives on quality of life after ICU have not been extensively explored.

Methods: Twelve participants from a 20 bed ICU at a tertiary referral hospital in regional Australia aged 23 to 86 years were enrolled into the study. All participants underwent daily sedation interruption (DSI). In-depth face-to-face interviews were conducted at two weeks after discharge and were repeated at eleven months. Data was thematically analysed.

Results: Overarching theme of being in limbo portraying existential uncertainty was found. Most of the participants suffered from amnesia and forgetfulness (Existential disruption). Others continued to experience nightmares, sleep deprivation and depression.

Conclusion: Surviving ICU is increasingly associated with cognitive impairment resulting in poor quality of life. DSI does not improve psychological outcomes. More research to establish the cause and treatment of cognitive impairment in ICU survivors is recommended.

248

Explanations Given by People with Epilepsy for Using Emergency Medical Services: A Qualitative Study

Ridsdale L., Virdi C., Noble A.

King's College, London, United Kingdom

Background: Approximately $50 \%$ of people with epilepsy (PWE) in the UK have had a seizure in the prior 12 months, and 13-18\% of PWE attend hospital Emergency Departments (ED) each year. However the majority of attendances are not regarded as clinically justified. This study describes PWE explanations for ED attendance based on interviews.

Methods: Semi - structured interviews were conducted with attenders recruited as a nested qualitative study that formed part of a cohort study based in three metropolitan hospitals. Interviews were tape recorded, transcribed and analysed thematically.

Results: Whilst prerequisite, a seizure alone was not their main explanation for attending ED. Explanations were contextual i.e. whether the seizure occurred at home or in a public place, and whether those nearby had knowledge, experience and confidence of what to do. Fears of sudden death by the PWE and others were additional reasons for use of emergency medical services.
Conclusion(s): Health care providers and policy makers can neglect the dilemma that PWE themselves often cannot make decisions themselves when they have a seizure as they are unconscious or confused. From the patients' perspective use of emergency services are appropriate, particularly when they are away from home, or do not have someone who knows about seizure management nearby. Significant others ability to manage was identified as important, as well as fear of death. Increasing proactive education amongst PWE and their significant others could potentially reduce ED use, and could be tested by means of a trial.

\section{Session P17: Ethnicity Issues}

\section{5}

\section{Juvenile and Adult-Onset ALS/MND Among Africans: Incidence, Phenotype, Survival: A Review}

\author{
Marin B. ${ }^{a}$, Kacem I. ', Diagana M. ', Gouider R. ${ }^{b}$, Preux P.M. ${ }^{d}$, \\ Couratier P. , TROPALS Group ${ }^{\mathrm{a}}$ \\ anstitut de Neurologie Tropicale Limoges - UMR Inserm1094 - \\ NeuroepidimiologieTropicale, France; ${ }^{\text {bDepartment of }}$ \\ Neurology Razi Hospital - La Manouba, Tunisia; ' $\mathrm{CHU}$ de

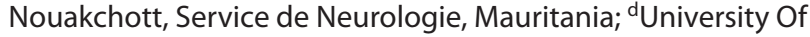 \\ Limoges, France
}

Background: The few studies looking at ALS in African populations were mostly case series varying in methods, sample sizes and collected data. Nevertheless, ethnic variation in ALS incidence and phenotype is of increasing interest. We reviewed the epidemiology of ALS in subjects of African origin, principally incidence, phenotype and prognosis.

Methods: We searched Medline, Scopus, Science direct, Bibliothèque Virtuelle de Neurologie Africaine (BVNA), (http:// www-ient.unilim.fr/) and African journal OnLine databases using the following search terms "amyotrophic lateral sclerosis (ALS)", "motor neuron disease (MND)" or "Charcot disease", in combination with "Africa", "ethnic groups", "blacks" or "epidemiology". Of 1264 references examined, 35 were included in this review.

Results: Among the 35 references, 19 studies were performed in the African continent and dealt with MND/ALS; four other studies focused on ALS-like syndromes; finally, 12 studies were not performed in Africa but focused on either incidence and mortality or survival of ALS in subjects of African origin. Several characteristics of ALS among Africans or subjects of African origin were identified: (i) lower incidence rates among people of African origin living in western countries, (ii) higher incidence of classic ALS among men, (iii) presence of juvenile form, (iv) younger age at onset of classic ALS. We cannot draw firm conclusions about (i) the prognosis in African ALS patients, (ii) prognostic factors, (iii) genetic or behavioral factors affecting incidence or clinical phenotype.

Neuroepidemiology 2012;39:177-283

Poster Presentations 
Conclusion(s): Further multicenter prospective studies with homogeneous methodological approaches need to be performed in Africa to clarify the situation. The TROPALS study is ongoing to contribute to answer remaining questions (www.tropals.unilim. fr).

\section{0 \\ Clinical Features of Iranian Americans with Multiple Sclerosis}

Eslami Farsani M., Amezcua L., Ledezma K.

University of Southern California, USA

Background: Recent decades have seen a substantial immigration of Iranians to the U.S., and there is limited information regarding their disease characteristics. We examine characteristics of multiple sclerosis (MS) in Iranian-Americans.

Methods: We performed a retrospective review of Iranian cases from Jan 2008-June 2012 at the Los Angeles County + University of Southern California Medical Center. Patients included had clinically definite MS based on McDonald's criteria and demographic and case-related information was obtained. Ethnic background was obtained and cases were classified as Iranian-Americans with and without Armenian background.

Results: Thirty-two cases met the clinical criteria of MS, with 17(53.1\%) of Armenian background. Female to male ratio was $3.2: 1$; mean age of first symptom was $29.7 \pm 8.2$ (range: 17-48); and $80 \%$ were on disease-modifying treatment. Relapsing Remitting (RR) MS was the most common form 29(90.6\%), with sensory symptoms as most frequent presentation ( $\mathrm{n}=12,35 \%)$, followed by optic neuritis $(\mathrm{n}=11,31 \%)$. There was no differences between proportion of $\mathrm{F}$ : $\mathrm{M}$ ratio and age of first symptom. Multifocal symptomatology $(n=6,35 \%)$ presentation was more commonly reported among Iranian-Armenians. Family history of MS was reported in 6 cases (19\%), with an even ethnic distribution.

Conclusion(s): Iranian Americans affected with MS share similar disease characteristics with previous reports. This study is the first to assess the significance behind the familial component on influencing the clinical presentation of MS in Iranian Americans. Iranian Americans with Armenian background presented with more frequent multifocal symptoms. Our study suggests that ethnicity should be carefully examined in future studies.

\section{Session P18: Public Health}

\section{1 Health-Related Quality of Life of Patients with
Parkinson's Disease: Multi-Center Study of the
Europastudy Group}

Winter $Y$.

Philipps-University Marburg, Germany

Background: Epilepsy is a devastating neurological disorder in which those afflicted can gain benefit from specific treatments based on their genetics and geographic location. Currently the prevalence of epilepsy is estimated at $0.5-3 \%$ of the world-wide population, and is increasing in developing countries.

Methods: In order to make more accurate assessments of the prevalence of epilepsy, we applied the capture-recapture method in a 12-month study of epilepsy in a population from the Sharur district of Nakhichevan Autonomous Republic, Azerbaijan with 114, 451 people from sixty-nine villages and towns. The following methods were applied to determine epilepsy: Door To Door (DTD) survey, Non-Medical Source (NMS), and Medical Source (MS).

Results: Using these three techniques, 1, 032 patients with epilepsy $(9.02 / 1000)$ were included in our study. The DTD survey determined 627 patients with epilepsy (5.48/1000) and 247 $(2.16 / 1000)$ from the NMS methods versus 158 (1.38/1000) from the MS methods. Applying the capture-recapture method (CRM), NMS, and DTD methods, the number of epilepsy cases increased to $1,330(11.62 / 1000)$ [95\% CI: 10.21-13.03]. The combinations of these methods show that 887 people $(7.75 / 1000)$ have a diagnosis of epilepsy.

Conclusion: Our conclusion is that, multiple epidemiological tasks used in our study can be used to estimate clinical signs and/ or markers in future applications for the determination and development of treatment strategies for this devastating disease in the post soviet countries such as the Nakhichevan Autonomous Republic of Azerbaijan. 
Aarseth, J.H. 220

Abad Diez, J. 243

Abd Al Wareth, A. 213, 253

Abdel-Hamid, I.A. 262

Abdelmonem, M. 253

Abdollahi, F. 199

Abdul-Latif, A. 234

Abdul-Latif, M. 234

Aboa-Eboulé, C. $\quad$ 196, 205, 238

Abrous, N. 231

Abu Bakar, M.R. 193

Ackah, F. 246

Adorni, F. 213

Ahmad, A. 270

Ahmane, H. 231

Akhtar, M. 270, 272

Albani, F. 251

Albers, K. 210

Alberti, D. 222

Alcaraz-Ochoa, R. 267

Aliev, G. 195

Allibert, R. 218, 260

Almeida, O. 252

Alonso, I. 223

Alperovitch, Y. 239

Alves, B.J. 237

Amber, S. 252, 254

Amezcua, L. 278

Amorim, G.S. 222

Anakhvir, S.G. 252

Andersen, O. 207

Andersen, P.A. 205

Anderson, N.E. 192

Angelova, A. 224

Angelov, B. 224

Ansari, M.A. 193

Apoil, M. 218, 260

Aqil, M. 270, 272

Ara Jose, R. 243

Arcolin, E. 255

ARCOS IV Programme

Collaborators 212

Arhab, D. 231

Aridon, P. 241

Arishima, T. 276

Arora, O.P. 252

Arriola, L. 206

Artero, S. 201, 209

Aruga, T. 276

Arveiler, D. 211

Asai, S. 238
Ashoo, G. 254

Atsuta, N. 241

Auriacombe, S. 210

Aw, D. 249

Badel, T. 232

Badry, N. 213, 215, 253

Badry, R. 213, 215, 253

Bahbiti, Y. 232, 242

Baird, D. 206

Bakaki, P. 215

Bakhti, S. 198

Bakshi, S.S. 252

Baldin, E. 251

Banack, S. 235

Banerjee, T. 180

Barber, P.A. 192

Barbot, C. 223

Barker-Collo, S. 203, 224, 243

Barker, R. 207

Baron, J.C. 218, 260

Barreto, S. 204, 237

Barros, J. 223

Barsic, I. 268

Bartko, D. 194, 228, 263

Baruzzi, A. 251

Bašić Kes, V. 232

Bauni, E. 200

Beghi, E. $185,186,231,239$, 257

Béjot, Y. $\quad 196,197,204,205$, 238, 263

Bela, S. 254

Belli, S. 222

Benaïm, C. 263

Benazzouz, B. 232, 242, 271

Benedetti, M.D. 203, 218

Benito-León, J. 211, 252

Bensenor, I. 204

Beral, V. 207

Berardelli, A. 269

Beridze, M. 274

Berkowitz, O. 201, 202

Bermejo-Pareja, F. 211, 250, 252

Bernhard, M. 230

Berr, C. 201

Bertorelli, R. 222

Beslac-Bumbasirevic, L. 238

Beugre, K. 196

Bezdolnij, J. 202

Bharucha, N.E. $\quad 184,187$
Bhattacharjee, M. 215, 217

Bianchi Maria, R. 203

Binquet, C. 205

Blair, A. 206

Blanco-Alvarez, V.M. $\quad 258,274$

Blauw, G.J. 221

Blazicek, P. 194

Blossom, S. 207

Boas, L. 242

Bocti, C. 208

Bodenant, M. 211

Bogdanov, E. 240

Bokhan, N. 270

Bollen, E. 221

Bonafé, A. 201, 209

Bonithon-Kopp, C. 259

Bonvicini, F. 255

Borges, E. 216

Bornstein, N. 181

Bottomley, C. 200

Bozina, N. 268

Bradley, M. 221

Brambila, E. 274

Brandes, A. 251

Brayne, C. $\quad 188,205,206,207$

Brickman, A. 201

Brown, A. 196

Bugnicourt, J.-M. 196

Burrows, E. 183

Caller, T. $\quad 235,246$

Calvet, D. 193

Campello, I. 243

Canoy, D. 207

Canto, C. 242

Caramelli, P. 204

Carol, B. 252

Carolina, S.M. 223

Caronti, B. 269

Carpay, H. 217

Carter, K. 192

Casillas, J.M. 263

Casseb, J. 243

Castañeda-Cisneros, G. 267

Catherine, Q. 196

Cerqueira, J. 236

Channaveerachari Naveen,

$$
\text { K. } 273
$$

Chantegret, C. 197

Chengo, E. 200

Chen, H. 184, 206, 214, 255

Chen, L. 195
Chi, A. 257

Chillon, J.-M. 196

Chinna, K. 193

Chiò, A. 239

Chuang Jimmy, M.-J. 242

Chunxue, W. 229

Cicin-Sain, L. 268

Clanet, M. 218, 249, 259

Clervoy, P. 225

Combor, I. 194

Confavreux, C. $\quad 218,249,259$

Cortese, A. 269

Cortese, L. 273

Cottin, Y. 196

Couratier, P. 277

Coutinho, P. 223

Cox, P. 235

Cox, R.R. 220

Crestani, A.M. 222

Crosby, C. 223

Cruz Vitor, T. 223

Cui, X. 266

Dahle, C. 207

Dai, H. 191

Dalal, P. 215, 217

Dalbem, Â. 244

Dalbem, J. 244

D’Alessandro, R. 251, 257

Dallongeville, J. 211

D’Amelio, M. 241

Danihel, L. 194

Darmency-Stamboul, V. 197

Daroch, C. 257

Dartigues, J.-F. 210

Das, S. 180

Daubail, B. 204

Daunt, L. 249

Davalos-Rodriguez, I. 267

Davalos-Rodriguez, N. 267

De Craen, A. 221

de Jorge, L. 267

Demirtas Raziye, N. 264

de Pasqua, S. 251

Devangi, D. 227

Devidze, E. 274

Dhanuka, A.K. 252

Diab, A.K. 265

Diagana, M. 277

Díaz-Guzmán, J. 250

Diehl, N. 196

Dikanović, M. 246 
Dimitrov, I. 245

Dimpfel, W. 234

Djaja, N. 258, 260

Dodel, R. 202, 258

Doijiri, R. 203

Doi, Y. 241

Dong, K. 216

Dover, D. 251

Doyle, P. 207

Drubics, K. 248

Ducimetière, P. 211

Dufouil, C. 237, 239

Dufour, M. 218

Durand, C. 197

Durastanti, V. 269

Durier, J. 238

Du, X. 183

Efe, B. 264

Egberts, T. 217

Eguibar, J.R. 258, 274

Elbaz, A. 210

El Hessni, A. 232, 242, 271

El Moselhy, E. 253

El Mrabet, F.Z. 271

Elrashidy, O. 261

El-Rashidy, O. 262

Emelyanov, A. 222

Emond, V. 208

Erdeljic, V. 268

Erro, E. 206

Esenay Figen, I. 276

Eslami Farsani, M. 278

Evans, A. 211

Fabcin, J. 194

Fagius, J. 207

Falip, E. 218

Fang, F. 250

Fang, J. 216

Farghaly, W. 213, 215, 253

Fegan, G. 200

Feigin, V. 203

Felix-Redondo, F. 247

Feng, H. 266

Ferdynus, C. 197

Fereshtehnejad, S.-M. 198

Fernández-Bergés, D. 247

Ferraz-Filho, J.R.L. 222

Ferrières, J. 211

Ferro, S. 251

Field, N. 235, 246

Figueiredo, J. 236

Filipets, O. 199

Filippini, G. 218, 257

Fink, M. 242, 243

Flemming, N. 225

Florim Greiciane, M. 222

Forsgren, L. 206
Foubert-Samier, A. 210

Fredrikson, S. 207

Freedman, N. 206

Freysz, M. 259

Fukuda, K. 275

Gabr, S. 262

Gagandeep, M. 252, 254

Gagandeep, S. 252

Gaifen, L. 216

Gajofatto, A. 203

Galeotti, F. 257

Gallacher, J. 207

Gallo, V. 205, 206, 207

Gangadhar Benagaluru, N. 273

Garancsi, G. 199

Garcia-Cruz, D. 267

Gasser, A.-I. 231

Gatzonis, S. 213

Gavrila Chervase, D. 206

Gawinecka, J. 232

Geml, J. 273

Georgoulopoulou, E. 255

Giatti, L. 204, 237

Gigineishvili, D. 201

Giménez-Muñoz, A. 243

Girardot-Tinant, N. 210

Giroud, M. 196, 197, 204, 205, 238, 263

Giussani, G. 239

Godefroy, O. 196

Golmakani, M.M. 198

Gombosova, Z. 194

Gomez Lira, M. 203

Gonzalez-Barrios, J.A. 258, 274

Gopalan, Y. 193

Górna, K. 272

Goswami, D. 272

Gouider, R. 277

Gouyon, J.-B. 197

Green, J. 207

Gregorio, M.L. 222

Gremeaux, V. 263

Group Lesvos 213

Grover, A. 252

Grzegorz, O. 256

Guerchet, M. 205

Guilland, J.C. 204

Guimaranes, J. 223

Gupta, S. 271

Gusev, E. 202

Hale, L. 265

Hamda, A. 233

Hamed, Y. 253

Hanam, R. 270

Hannan, A. 183

Hansson, O. 206
Han, Y. 270

Han, Y.-Y. 202

Hao, Z. 197

Hardiman, O. 239

Hartnett, E. 268

Hasanov, E. 195

Hasanov, N. 195

Hashimoto, M. 276

Hassan, M. 253

Haynes, H. 221, 223

Helmer, C. 210

Heng, Z. 229

Henigsberg, N. 268

Henrotin, J.-B. 196

Hermann, P. 232

Hernández-Gallego, J. 250

Hernández-Ramos, F. 247

Herrero-San Martín, A. 252

Hervieu, M. 204

Higgins, I. 277

Hillbom, M. 254

Hillert, J. 207

Hill, M. 251

Hiroaki, S. 275

Hiroshi, F. 255

Hiroto, F. 255

Hollenbeck, A. 206

Holleran, K. 262

Hong, Z. 255

Honig, H. 240

Hopkins, K. 223

Huang, X. 206

Huisman, M. 252

Ighanian, B. 247

Ikram, A. 179

Ishihara, L. 207

Isler, A. 276

Ismail, M.V. 270

Ivashynka, A. 245

Iyer, A. 201

Izumi, M. 240

Jacques, P. 214

Jacquin, A. 204, 205, 238

Jalilzadeh, G. 198

Jamali Danesh, Y. 198

Jaracz, K. 272

Jaseja, B. 267

Jaseja, H. 267

Jaseja Harinder 219

Jasem, Z. 262

Jayalakshmi, S. 233

Jeetamol, S.G. 252

Jeyaraj, D.P. 254

Jiahe, X. 266

Jiang, B. 191, 193

Jones, K. 203

Jung-Wan, K. 229, 235
Junji, T. 255

Juvela, S. 209, 254

Jyoti, M. 227

Kacem, I. 277

Kadojić, D. 246

Kadojić, M. 246

Kah Hay, Y. 193

Kaiboriboon, K. 215

Kalamatianos, T. 213

Kalenska, D. 194

Kamel, F. 250

Kandil, M. 213, 215, 253

Karch, A. 211, 232

Kartsivadze, S. 201

Karuppusamy, A. 269

Katunina, E. 202

Kaur, M. 252

Kaur, P. 254

Kazungu, M. 200

Kebapci, N. 264

Kee, F. 211

Kengeri Kishore, K. 273

Ken, M. 255

Kensuke, K. 275

Kern, J. 232

Kesselring, J. 190

Khanam, R. 272

Khan Nurzalina, A.K. 193

Khuroo Arshad, H. 272

Kisic-Tepavcevic, D. 238

Kjeld, A. 225

Kleinschmidt, I. 200

Klobucka, S. 263, 264

Koizia, A. 233

Koizia, L. 233

Kokubo Y. 203

Kombe, M. 200

Konishi, J. 221

Koroukian, S. 215

Kovac, M. 264

Krajewski-Siuda, K. 256

Krishnamurthi, R. 212

Kroger, E. 208

Krolak-Salmon, P. 226

Krolo, I. 232

Kudumallige Suresh, K. 273

Kurian, K. 221, 223

Kurtzke, J. 190

Kurva, E. 194

Kuwahara, H. 255

Kyoshima, K. 240

Kyrozis, A. 205, 206

Labanczova, L. 194

Lakhrissi, B. 271

Lalkaka, J. 184

La Mantia, L. 218

Lamy, C. 196

Landtblom, A.M. 207 
Lauer, K. 236

Lawes, C. 224

Le Bars, E. 201, 209

Ledezma, K. 278

Le Goff, M. 210

Lehto, H. 209

Leibson, C. 196

Leijten, F. 217

Lellis, B. 216

Lelovics, Z. 248

Leon-Chavez, B.A. 258, 274

Leone, M. 245

Lesieur, S. 224

Liczbińska, G. 272

Lifen, C. 264

Li, G. 195

Liheng, B. 229

Liu, L. 216

Liu, M. 197, 266

Liu, R. 206

Logroscino, G. $\quad 239,257$

Lojko, D. 272

Lolk, A. 225

López Gastón, J. 243

Lo Re, M. 241

Lo Re, V. 241

Lorgis, L. 196

Loufardaki, M. 213

Louis, E. 181,185

Loureiro, J.L. 223

Lozano-Mera, L. 247

Lucenti, A. 245

Ludolph, A. 208

Lunsford, L.D. 201, 202

Luo, W. 195

Lv, H. 195

Lycke, J. 207

Magalov, S. 195

Magosso, E. 193

Mahmood, D. 272

Maia, P. 236

Maier, I. 221

Malec, J. 196

Malik, M.A. 230

Maller, J. 201, 209

Malmestrom, C. 207

Mandaville, G.D. 254

Mandrekar, J. 196

Mandrioli, J. 255

Marin, B. 277

Marincola, F. 269

Markeljevic, J. 268

Marques Neves, J. 223

Marta, J. 243

Martin, C. 207

Martinez-Fong, D. 258, 274

Martínez-Salio, A. 250

Martins, D.P. 222
Martynyuk, A. 226

Marty, R. 226

Masala, G. 206

Mas, J.-L. 192, 218, 260

Massacesi, L. 218

Massari, M. 257

Mazda, J. 205

Mazoyer, B. 239

Mazzola, M.A. 241

Mbelesso, P. 205

Mbuba, C. 200

McPherson, K. 243

Medel, A. 252

Meder, J.-F. $\quad 218,260$

Meenakshi, S. 252, 254

Meguro, K. 237

Mehrabani, M. 198

Mei, N. 195

Mejean, N. 197

Mekhail, M. 261

Mello, S.R. 216

Mesfioui, A. 232, 242, 271

Meslin, C. 201, 209

Messina, M. 231, 240

Messina, P. 239

Metcalf, J. 235

Michalke, B. 255

Michel, P. 216

Middelkoop, H. 221

Middleton, L. 206

Mikhailov, I. 240

Milanese, C. 218

Milanov, I. 245

Millan-Nuñez, V. 247

Millefiorini, E. 269

Millul, A. 240

Ming, L. 182

Mirek, S. 259

Mitchell, D. 239

Miyamoto, Y. 203

Miyashita, K. 203

Mo, C. 183

Mohammadi, N. 198

Monaco, A. 269

Montaye, M. 211

Moraes, L. 243

Moran-Moguel, C. 267

Moreno, T. 211

Morita, M. 241

Morris, M. 214

Mortamais, M. 201, 209

Mouanga, A. 205

Moutaouakil, F. $\quad 232,242$

Mozhayev, S. 220

Mujeeb, M. 270, 272

Munakata, M. 275

Muraskin, J. 201

Musicco, M. 213

Mutsufusa, W. 255

Myhr, K.M. 220
Nadar, M. 262

Nagatsuka K. 203

Nagel, G. 208

Nagiub, H. 213, 215, 253

Nagy, F. 248

Najmi, A.K. $\quad 270,272$

Nakache, R. 271

Nakagawa, E. 224

Nakano, I. 241

Nakashima, K. 244

Nakashita, S. 244

Nakazone, M.A. 222

Naldi, P. 245

Nali, L. 243

Naritomi, H. 203

Nassar, M. 262

Nastbakken, J.K. 220

Nater, U. 258

Ndamba Bandzouzi, B. 205

Nelson, L. 188

Nerman, O. 207

Nesaretnam, K. 193

Newton, C. 200

Ng Bee, H. 193

Ngugi, A. 200

Niklas, J. 271

Nishida, Y. 238

Noble, A. 277

Norrving, B. $\quad 183,186$

Nosal, V. 194

Ntaios, G. 216

Nuñez-Reveles, N. 267

Odin, P. 240

O'Donnell 180

Oertel, W. 202, 258

Oh Shin, J. 229

Ok, S. 234

Okudera, H. 276

Oláh, T. 248

Olberg, H.K. 220

Olival, G. 243

Omneya, I.Y. 261

Oppenheim, C. 218,260

Opprecht, N. 259

Orozco-Gutierrez, M.H. 267

Osseby, G.V. $\quad 205,238$

Ouichou, A. 232, 242, 271

Óváry, C. 199

Özbey, H. 275

Pakdaman, H. 198

Pałys, W. 272

Pandian, J.D. 189, 252

Pang, T. 183

Panico, S. 206

Pannuti, C. 242

Papaiz-Alvarenga, R.M. 236, 244

Papantonio, A.M. 231
Papavasileiou, V. 216

Papp, Z. 248

Paramdeep, K. 252

Parimelazhgan, T. 269

Parker, V. 277

Park, Y. 206

Passante, N. 218

Passos, V. 204, 237

Pawar, S.S. 252

Pchelina, S. 222

Pearce, N. 205

Pekmezovic, T. 238

Pelosse, M. 210

Penalva, A. 242, 243

Pereira, P. 236

Perez, F. 210

Perkins, P. 196

Peters, T. 250

Pezzella, F.R. 225

Pfleiderer, S. 240

Piehl, F. 207

Pillai, K.K. 272

Piña-Leyva, C. 258, 274

Pinhel, M. 222

Pisoni, N. 222

Pniewska, J. 272

Ponto, C. 232

Porras, M. 209

Portet, F. 201

Poulain, V. 209

Poussa, K. 209

Preux, P.M. 205, 239, 244, 277

Pridmore, H. 192

Prina, M. 252

Prinelli, F. 213

Provenzano, F. 201

Pugliatti, M. 257

Puljula, J. 254

Pupillo, E. 239, 240

Qi, H. 270

Qi, X. 195

Qorbani, M. 248

Quesenberry, C. 210,247

Rageh, T. 213, 215, 253

Ragonese, P. 241

Ramirez-Garcia, S. 267

Ramírez-Moreno, J. 247

Ransome, M. 183

Ransom, J. 196

Raschetti, R. 257

Rasiah, S. 262

Raymkulova, O. 245

Realmuto, S. 241

Regio, V. 231

Renoir, T. 183

Reynes, C. 201

Riboli, E. 206

Ridsdale, L. 277 
Ritchie, K. 201, 209

Rivas, R. 257

Roberts, S. 192

Rocca, W. 180, 182

Rocher, F. 218

Rochette, L. 208

Rodrigues, M. 216

Rodrigues Santos, C.E. 236

Romano, C. 243

Rong, J. 270

Rosenbohm, A. 208

Rosenfelt, K. 233

Rossier, P. 231

Rossi Silva, S.D. 222

Rostohar Bijelić, B. 246

Rothenbacher, D. 208

Rouaud, O. 205

Rousseau, P.F. 225

Roze, S. 226

Ruano, E. 210

Ruano, L. 223

Ruggerone, S. 245

Ruidavets, J.-B. 211

Ruikar, D. 233

Runmarker, B. 207

Rusnak, F. 194

Russo, A. 213

$\mathrm{Ru}, \mathrm{X} . \quad 191,193$

Sabatier, R. 201

Sacerdote, C. 206

Sagot, P. 197

Sajedi Seyed, A. 199

Sakamoto, M. 276

Sakamoto, T. 276

Sakas, D 213

Salemi, G. 241

Salviati, A. 203, 222

Sambricio, J. 211

Sameniene, J. 265

Sanchez-Corona, J. 267

Sánchez-Ferro, Á. 250

Sanchez-Garcia, D.M. 267

Sanderson, M. 246

Sandler, D. 250

Sanja, M. 238

San Nicolas-Fernandez, H. 267

Santos, M.L.T. 222

Santuccio, C. 257

Sarac, H. 268

Satizabal, C. 237

Savettieri, G. 241

Savić Pavičin, I. 232

Saxena, A. 252

Schiltz, N. 215

Schopflocher, D. 208, 212

Schwarzman, A. 222

Sehgal, H. 252

Sequeiros, J. 223

Serrano, P. 223
Sertic, J. 268

Seung-In, K. 235

Shaaban, I. 253

Shaaban, S. 262

Shah, B. 252

Shahbeigi, S. 198

Shakarishvili, R. 201

Shalashvili, G. 201

Shanidze, L. 274

Shan, J. 210, 247

Sharma, A. 254, 257

Sharma, J. 249

Shatla, R. 261

Shatla, R.H. 262

Shavinder, S. 254

Shegelia, N. 201

Shehata, G. 213, 215, 253

Shintaku, H. 250

Shi, X. 246

Shlomo Yoav, B. 207

Short, A. 183

Shrivastava, M. 275

Shuaib, I.L. 193

Shufeng, Z. 229

Shweta, J.V. 254

Siebel, P. 252

Sieri, S. 206

Sierra-Hidalgo, F. 250

Silva, S. 222

Silveira, I. 223

Simiao, W. 266

Simko, M. 231

Simon, P.Y. 225

Singh, Y. 252

Siqueira, H. 216, 244

Skoog, B. 207

Soaham, D. 227

Sobti, M.K. 252

Sobue, G. 241

Solari, A. 218

Solovjev, N. 255

Sorrentino, R. 225

Soto-Rodriguez, G. 258, 274

Soumare, A. 239

Spencer, P. $\quad 179,191$

Starkey, N. 203, 224, 243

Stawiarz, L. 207

Stefani, A. 203

Stepniak Iwona 268

Stommel, E. $\quad 235,246$

Stranjalis, G 213

Sudlow, C. 207

Sumita, L. 242, 243

Sundram, K. 193

Sun, H. 193

Surath, M. 233

Susanne, H. 271

Suwalska, A. 272

Suwalski, J. 272

Suzuki, K. 240
Svenson, L. 208, 212, 246, 251

Swiat, M. 256

Szabó, G. 199

Szucs, A. 199

Tabarean, J. 269

Tabei, R. 275

Tadayyon, S. 198

Taioli, F. 222

Takahashi, Y. 238

Takahide, A. 275

Talbott, E. 201, 202

Tanaka, K. 244

Tan, C. 264

Tao, W. 197

Tataru, A.M. 218, 260

Tavakoli, A. 247

Taylor Bruce 189

Tchouata Ngandjouc, L. 196

Tedeholm, H. 207

Tembo, A.C. 277

Testoni, S. 251

Tetri, S. 254

Teucher, B. 206

Theadom, A. 203, 243

Thirthahalli, J. 273

Thomas, S. 211

Tison, F. 210

Tognola Waldir, A. 222

Torres, F. 216

Torres-Soto, M. 274

Touchon, J. 201, 209

Touze, E. 218, 260

Tramacere, I. 218

Traykov, L. 245

Trincado, R. 211, 250

Trocello, J.M. 210

Troisgros, O. 263

TROPALS Group 277

Tsiskaridze, A. 201

Tuna, A. 223

Turatti, M. 203

Turc, G. 218, 260

Tzavara, C. 213

Tzourio, C. $182,237,239$

Udgeeth, T. 227

Udupi, A. 273

Udupi Shashank, A. 273

Ugarte, A. 258, 274

Uijl, S. 217

Ünal, H. 208

Uno, H. 203

Vaaramo, K. 254

Vainchtock, A. 226

Vairale, J. 215, 217

Valery, F. 224, 243

Vanacore, N. 205, 206

Van Buchem, M. 221
Van Den Eeden, S. 210, 247

Van der Grond, J. 221

Van Der Linden, P. 217

van Es, A. 221

Van Ganse, E. 218

Vanier, M.T. 187

Varalakshmi, E. 233

Varga, C. 248

Varga-Győrfi, K. 248

Varma, R. 233

Varsha, P. 224

Varzaityte, L. 265

Vasconcelos, R. 236

Vasquez, M. 257

Vazzoler, G. 241

Vedeler, C.A. 220

Vega, S. 211

Velehorschi, C. 273

Venkatesh, K. 192

Verma, S. 252

Vermeleun, R. 206

Vermeulen, R. 205

Vidal, J. 242, 243

Vilela Gomes, B. 216

Villarejo, A. 211

Villella, G. 273

Vinceti, M. 255

Virdi, C. 277

Vivekanandhan, S. 275

Volov, M. 220

Volpini, V. 267

Vrethem, M. 207

Vukusic, S. 218, 249, 259

Wada-Isoe, K. 244

Wagner, A. 211

Wakasugi, M. 276

Wang, C. 216

Wang, D. 197

Wang, D.Z. 216

Wang, E. 269

Wang, F. 195

Wang, H. 214

Wang, J. 255

Wang, W. 191, 193

Wang, Y. 216

Wark, P.A. 205

Warren, S. 208

Wassenaar, M. 207, 217

Waters, D. 265

Weibull, C. 250

Westendorp, R. 221

Westergaard, M.L. 248

Wiebers, D.O. 179

Williams, M. 223

Winter, Y. 202, 258, 278

Woimant F. 210

Wong Jia, W. 193

Wu, S. 191, 193 
Xiao, J. 195

Xu, J. 271

Yakimovskii, A. 222

Yamawaki, M. 244

Yanagisawa, H. 266

Yang, C. 195

Yang, G. 255
Yashpal, S. 254

Ye, W. 250

Yuan, L. 255

Yu, H. 255

Yusuke, U. 244

Zabbarova, A. 240

Zadravec, D. 232
Zamani, B. 198

Zerr, I. 232

Zhang, Q. 229

Zhang, Y. 221

Zhao, J. 255

Zhao, X. 216, 229

Zheng, H. 216

Zhou, F. 195
Zhu, Q. 266

Zhu, Y.-C. 237, 239

Ziakova, E. 263, 264

Zidanes Lira, A. 222

Zielicka, Z. 233 